\title{
Evidence-based clinical guidelines for immigrants and refugees
}

\author{
Kevin Pottie MD MCISc, Christina Greenaway MD MSc, John Feightner MD MSc, Vivian Welch MSc PhD, \\ Helena Swinkels MD MHSc, Meb Rashid MD, Lavanya Narasiah MD MSc, Laurence J. Kirmayer MD, \\ Erin Ueffing BHSc MHSc, Noni E. MacDonald MD MSc, Ghayda Hassan PhD, Mary McNally DDS MA, \\ Kamran Khan MD MPH, Ralf Buhrmann MDCM PhD, Sheila Dunn MD MSc, Arunmozhi Dominic MD, \\ Anne E. McCarthy MD MSc, Anita J. Gagnon MPH PhD, Cécile Rousseau MD, Peter Tugwell MD MSc; \\ and coauthors of the Canadian Collaboration for Immigrant and Refugee Health
}

Competing interests: See end of document for competing interests.

Coauthors of the Canadian Collaboration for Immigrant and Refugee Health: Deborah Assayag, Elizabeth Barnett, Jennifer Blake, Beverly Brockest, Giovani Burgos, Glenn Campbell, Andrea Chambers, Angie Chan, Maryann Cheetham, Walter Delpero, Marc Deschenes, Shafik Dharamsi, Ann Duggan, Nancy Durand, Allison Eyre, Jennifer Grant, Doug Gruner, Sinclair Harris, Stewart B. Harris, Elizabeth Harvey, Jenny Heathcote, Christine Heidebrecht, William Hodge, Danielle Hone, Charles Hui, Susan Hum, Praseedha Janakiram, Khairun Jivani, Tomas Jurcik, Jay Keystone, Ian Kitai, Srinivasan Krishnamurthy, Susan Kuhn, Stan Kutcher, Robert Laroche, Carmen Logie, Michelle Martin, Dominique Elien Massenat, Debora Matthews, Barry Maze, Dick Menzies, Marie Munoz, Félicité Murangira, Amy Nolen, Pierre Plourde, Hélène Rousseau, Andrew G. Ryder, Amelia Sandoe, Kevin Schwartzman, Jennifer Sears, William Stauffer, Brett D. Thombs, Patricia Topp, Andrew Toren, Sara Torres, Ahsan Ullah, Sunil Varghese, Bilkis Vissandjee, Michel Welt, Wendy Wobeser, David Wong, Phyllis Zelkowitz, Jianwei Zhong, Stanley Zlotkin.

Editor's note: See Appendix 1, available at www.cmaj .ca/lookup/suppl/doi:10.1503/cmaj.090313/-/DC1, for affiliations and contributions of coauthors.

This document has been peer reviewed.

Correspondence to: Dr. Kevin Pottie, kpottie@uottawa.ca

CMAJ 2011. DOI:10.1503/cmaj.090313
- KEY POINTS

- Clinical preventive care should be informed by the person's region or country of origin and migration history (e.g., forced versus voluntary migration).

- Forced migration, low income and limited proficiency in English or French increase the risk of a decline in health and should be considered in the assessment and delivery of preventive care.

- Vaccination (against measles, mumps, rubella, diphtheria, tetanus, pertussis, polio, varicella, hepatitis B and human papillomavirus) and screening (for hepatitis B, tuberculosis, HIV, hepatitis C, intestinal parasites, iron deficiency, dental pain, loss of vision and cervical cancer) should be routinely provided to at-risk immigrants.

- Detecting and addressing malaria, depression, posttraumatic stress disorder, child maltreatment, intimate partner violence, diabetes mellitus and unmet contraceptive needs should be individualized to improve detection, adherence and treatment outcomes.

\section{Conditions covered in systematic reviews}

(see Appendix 2, available at www.cmaj.ca/lookup/suppl/doi:10.1503/cmaj.090313/-/DC1 for summary of recommendations and clinical considerations)

\section{Infectious diseases}

- Measles, mumps, rubella

- Diphtheria, tetanus, polio, pertussis

- Varicella

- Hepatitis B

- Tuberculosis

- HIV

- Hepatitis C

- Intestinal parasites (Strongyloides and Schistosoma)

- Malaria

\section{Chronic and noncommunicable diseases}

- Diabetes mellitus

- Iron-deficiency anemia

- Dental disease

- Vision health

Women's health

- Contraception

- Cervical cancer

- Pregnancy

\section{Mental health and maltreatment}

- Depression

- Post-traumatic stress disorder

- Child maltreatment

- Intimate partner violence 


\section{Overview: evidence-based clinical guidelines for immigrants and refugees}

There are more than 200 million international migrants worldwide, ${ }^{1}$ and this movement of people has implications for individual and population health. ${ }^{2}$ The 2009 United Nations Human Development Report ${ }^{3}$ suggested that migration benefits people who move, through increased economic and education opportunities, but migrants frequently face barriers to local health and social services. In Canada, international migrants are a growing ${ }^{4}$ and economically important segment of the population (Table 1A)..$^{5-8}$

Immigrants to Canada are a heterogeneous group. Upon arrival, new immigrants are healthier than the Canadian-born population, both because of immigrant-selection processes and policies and because of sociocultural aspects of diet and health behaviours. However, there is a decline in this "healthy immigrant effect" after arrival. ${ }^{5}$ In addition, compared with the Canadian-born population, subgroups of immigrants are at increased risk of disease-specific mortality; for example, Southeast Asians from stroke (odds ratio [OR] 1.46, 95\% con-

Table 1A: Classification of international migration to Canada (2007)*

\begin{tabular}{|c|c|}
\hline Immigration category & $\begin{array}{l}\text { Annual } \\
\text { migration } \\
\text { (no.)t‡ }\end{array}$ \\
\hline \multicolumn{2}{|l|}{ Permanent residents ${ }^{6}$} \\
\hline $\begin{array}{l}\text { Economic class (business and economic } \\
\text { migrants) }\end{array}$ & 131000 \\
\hline Family class (family reunification) & 66000 \\
\hline $\begin{array}{l}\text { Humanitarian class (refugees resettled from } \\
\text { abroad or selected in Canada from refugee } \\
\text { claimants) }\end{array}$ & 28000 \\
\hline Others & 11000 \\
\hline Total & 237000 \\
\hline \multicolumn{2}{|l|}{ Temporary residents $^{6}$} \\
\hline Migrant workers & 165000 \\
\hline International students & 74000 \\
\hline $\begin{array}{l}\text { Refugee claimants (those arriving in Canada } \\
\text { and claiming to be refugees) }\end{array}$ & 28000 \\
\hline Other temporary residents ${ }^{6}$ & 89000 \\
\hline Total $^{6}$ & 357000 \\
\hline \multicolumn{2}{|l|}{ Other migrants } \\
\hline $\begin{array}{l}\text { Total irregular migrants, } \S \text { not annual } \\
\text { migration }^{7}\end{array}$ & $\sim 200000$ \\
\hline Visitors $^{8}$ & $\sim 30100000$ \\
\hline
\end{tabular}

*Reproduced, with permission, from Gushulak et al. ${ }^{5}$

†Numbers rounded to nearest 1000 . Total in each category may not match sum of values reported because of rounding.

¥Unless otherwise indicated.

§No official migration status; this population includes those who have entered Canada as visitors or temporary residents and remained to live or work without official status. It also includes those who may have entered the country illegally and not registered with authorities or applied for residence. fidence interval [CI] 1.00-1.91), ${ }^{9}$ Caribbeans from diabetes mellitus (OR 1.67, 95\% CI 1.03-2.32) and infectious diseases (e.g., for AIDS, OR 4.23, 95\% CI 2.72-5.74), and immigrant men from liver cancer (OR 4.89, 95\% CI 3.29-6.49). ${ }^{10}$

The health needs of newly arriving immigrants and refugees often differ from those of Canadian-born men, women and children. The prevalence of diseases differs with exposure to disease, migration trajectories, living conditions and genetic predispositions. Language and cultural differences, along with lack of familiarity with preventive care and fear and distrust of a new health care system, can impair access to appropriate health care services. ${ }^{11}$ Additionally, patients may present with conditions or concerns that are unfamiliar to practitioners. ${ }^{5,10}$

Many source countries have limited resources and differing health care systems, and these differences may also contribute to health inequalities among migrants. ${ }^{12}$ In these guidelines, we refer to low-and middle-income countries as "developing."

\section{Why are clinical guidelines for immigrants needed?}

Canadian immigration legislation requires that all permanent residents, including refugees, refugee claimants and some temporary residents, undergo an immigration medical examination. Screening is undertaken to assess the potential burden of illness and a limited number of public health risks. The examination is not designed to provide clinical preventive screening, as is routinely performed in Canadian primary care practice, and it is linked to ongoing surveillance or clinical actions only for tuberculosis, syphilis and HIV infection. ${ }^{5}$

The Canadian Task Force on Preventive Health Care and the US Preventive Services Task Force have produced many high-quality clinical prevention recommendations, but these statements have not explicitly considered the unique preventive needs and implementation issues for special populations such as immigrants and refugees. Evidence-based recommendations can improve uptake and health outcomes related to preventive services, even more so when they are tailored for specific populations. ${ }^{13}$

\section{How are these guidelines different?}

Use of evidence-based methods has yet to substantially affect the field of migration medicine. ${ }^{14}$ The Canadian Collaboration for Immigrant and Refugee Health explicitly aims to improve patients' health using an evidence-based clinical preventive approach to complement existing public health approaches. In selecting topics, primary care practitioners considered not just the burden of illness but also health inequities and gaps in current knowledge. ${ }^{15}$ Public health concerns and predeparture migrant screening and treatment protocols were also consid- 
ered, but these were not the driving force for the recommendations. We implemented evidence-based methods, which included searches for evidence on immigrant preferences and values, as well as incorporating the GRADE approach (Grading of Recommendations Assessment, Development and Evaluation), to formulate clinical preventive recommendations. ${ }^{16-18}$ Our evidence reviews synthesized data from around the world, and our recommendations focus on immigrants, refugees and refugee claimants, with special attention given to refugees, women and the challenges of integrating recommendations into primary care. Migrants living without official status are particularly vulnerable, but specific evidence for this population is limited. ${ }^{19}$ In these guidelines, the "health settlement period" refers to the first five years of residence in Canada for an immigrant or refugee, the time during which loss of the healthy immigrant effect begins to surface.

In recent years, there has been an increase in development of practice guidelines for international migrants. ${ }^{20}$ Notable publications have included Cultural Competency in Health, ${ }^{21}$ Immigrant Medicine ${ }^{22}$ and guidelines for refugees from the Australasian Society for Infectious Diseases..$^{23}$ Many have been designed to address diseases and conditions of public health importance, ${ }^{23-25}$ and some highlight the importance of psychosocial problems and mental illness, issues of women's health and chronic noninfectious diseases. ${ }^{21,26,27}$ Other practice guidelines include strategies to improve communication (e.g., interpreters), responsiveness to sociocultural background (e.g., cultural competence), empowerment (e.g., health literacy), monitoring (e.g., health and access disparities) and strategies for comprehensive care delivery. ${ }^{21}$

Our recommendations differ from other guidelines because

Box 1A: Fourteen-step process for evidence reviews used by the Canadian Collaboration for Immigrant and Refugee Health

1. Develop clinician summary table

2. Develop logic model and key questions

3. Set the stage for admissible evidence (using search strategy)

4. Assess eligibility of systematic reviews

5. Search for data specific to immigrant and refugee populations

6. Refocus on key clinical preventive actions and key questions

7. Assess quality of systematic reviews

8. Search for evidence to update selected systematic reviews

9. Assess eligibility of new studies

10. Integrate data from updated search

11. Synthesize final evidence bank and draft two key clinical actions

12. Develop table for summary of findings

13. Identify gaps in evidence and needs for future research

14. Develop clinical preventive recommendations using GRADE (Grading of Recommendations Assessment, Development and Evaluation)

*Adapted, with permission, from Tugwell and others. ${ }^{16}$ of our insistence on finding evidence for clear benefits before recommending routine interventions. For example, in our guidelines for post-traumatic stress disorder, intimate partner violence and social isolation in pregnancy, we recommend not conducting routine screening, but rather remaining alert. With regard to screening for asymptomatic intestinal parasites, we recommend focusing on serologic testing for high burden of disease parasites, rather than traditional testing of stool for ova and parasites.

\section{How were these guidelines developed?}

We followed the internationally recognized Appraisal of Guidelines for Research and Evaluation (AGREE; www.agreetrust.org). We selected guideline topics using a literature review, stakeholder engagement and the Delphi process with equity-oriented criteria. ${ }^{15}$ In May 2007, we held a consensus meeting of experts in immigrant and refugee health to develop a systematic process for transparent, reproducible, evidence-based reviews. The guideline committee selected review leaders from across Canada on the basis of their clinical and evaluation expertise (see Appendix 1, available at www.cmaj.ca/lookup/suppl/doi:10.1503/cmaj.090313/-/DC1).

The 14-step evidence review process (Box 1A) ${ }^{16}$ used validated tools to appraise the quality of existing systematic reviews, guidelines, randomized trials and other study designs. We searched MEDLINE, Embase, the Cochrane Library and other sources for admissible evidence, specifically reviews and related studies, from 1996 to 2010. We identified guidelines developed by other groups but based our recommendations on evidence from primary studies. We identified patientimportant outcomes and used the GRADE approach to assess the magnitude of effect on benefits and harms and on quality of evidence. We included both direct evidence from immigrant and refugee populations and indirect evidence from other populations. We downgraded the quality of evidence for indirectness when there was concern that the evidence might not be applicable to immigrant and refugee populations (e.g., because of differences in baseline risk, morbidity and mortality, genetic and cultural factors, and compliance variations). We assessed whether benefits outweighed harms, the quality of evidence, and values and preferences to minimize the potentially negative effects of labelling on patients, families and communities (Table 1B). ${ }^{16-18}$

Each of the resulting evidence reviews for priority conditions of the Canadian Collaboration for Immigrant and Refugee Health provides detailed methods and results concerning the burden of illness for the immigrant populations relative to Canadian-born populations, along with information about effectiveness of screening and interventions, a discussion of clinical considerations, the basis for recommendations and gaps in research.

\section{How should I begin to assess immigrants for clinical preventive care?}

Determine each person's age, sex, country of origin and migra- 
tion history to tailor preventive care recommendations. In caring for socially disadvantaged populations, sequencing of care using checklists or algorithms can improve both the uptake and the delivery of preventive care ${ }^{13}$ and allows other members of the primary health care team to participate in the delivery of care. Working with interpreters, cultural brokers, patients' families and community support networks can support culturally appropriate care..$^{28}$ Most importantly, clinicians should recognize that the implementation of recommendations (vaccinations, for example) may take three or four visits, a process more akin to the delivery of well-baby care than to an annual examination. Our recommendations are aimed at primary care practitioners, but competencies related to immigrant and crosscultural care will vary depending on training and experience, and expert support should be sought accordingly.

\section{Which immigrant populations face the most significant health risks?}

Refugees, who are by definition forcefully displaced, are at highest risk for past exposure to harmful living conditions, violence and trauma. Refugees undergo medical screening before admission to Canada but are protected by law from exclusion on the basis of noninfectious burden of illness (through the Immigration and Refugee Protection Act). ${ }^{29}$ The health risks of refugees and other migrants vary greatly depending on exposures (e.g., to vectors of disease such as mosquitos), trauma from war, living conditions (e.g., access to water and sanitation), neglect from long periods in refugee camps, susceptibilities (e.g., related to ethnicity and migration stress), social stratification (e.g., race, sex, income, education and occupation) and access to preventive services (e.g., predeparture access to primary care, vaccinations and screening, access to Canadian services and access issues related to linguistic and cultural barriers).

Specifically, refugees are at risk for a rapid decline in selfreported health after arrival (OR 2.31, 95\% CI 1.1-4.9), as are

Table 1B: Basis of recommendations*

\begin{tabular}{ll}
\hline Issue & \multicolumn{1}{c}{ Process considerations } \\
\hline $\begin{array}{l}\text { Balance between } \\
\text { desirable and } \\
\text { undesirable effects }\end{array}$ & $\begin{array}{l}\text { Those with net benefits or trade- } \\
\text { offs between benefits and harms } \\
\text { were eligible for a positive } \\
\text { recommendation }\end{array}$ \\
Quality of evidence & $\begin{array}{l}\text { Quality of evidence was classified } \\
\text { as "high," "moderate," "low" or } \\
\text { "very low" on the basis of } \\
\text { methodologic characteristics of } \\
\text { available evidence for a specific } \\
\text { clinical action }\end{array}$ \\
Values and preferences & $\begin{array}{l}\text { Values and preferences refer to } \\
\text { the worth or importance of } \\
\text { health state or consequences of } \\
\text { following a particular clinical } \\
\text { action }\end{array}$ \\
\hline
\end{tabular}

* Reproduced, with permission, from Tugwell et al. ${ }^{16}$ Based on GRADE (Grading of Recommendations Assessment, Development and Evaluation. ${ }^{17}$ low-income immigrants (OR 1.5, 95\% CI 1.3-1.7) and immigrants with limited English- or French-language proficiency. ${ }^{5,30-35}$ There is also an increased risk of reporting poor health among immigrants with limited English- or Frenchlanguage proficiency (OR 2.0, 95\% CI 1.5-2.7), those facing cost-related barriers to health care (OR 2.8, 95\% CI 1.7-4.5), ${ }^{36}$ low-income immigrants ${ }^{32}$ and non-European immigrants (OR $2.3,95 \%$ CI $1.6-3.3) .^{31}$

\section{Clinical recommendations}

Considering the burden of illness of immigrant populations, the quality of evidence for screening and interventions, and the feasibility of clinicians implementing the recommendations, we have organized our recommendations into four groups: infectious diseases, mental health and physical and emotional maltreatment, chronic and noncommunicable diseases, and women's health.

Appendix 2 (available at www.cmaj.ca/lookup/suppl /doi:10.1503/cmaj.090313/-/DC1) summarizes the evidence review and recommendations for each topic, providing specific comments about how the number needed to screen and treat for net benefits would differ for immigrant populations.

\section{Infectious diseases}

Many immigrants are susceptible to vaccine-preventable diseases upon arrival in Canada. For example, 30\%-50\% of new immigrants are susceptible to tetanus, ${ }^{37} 32 \%-54 \%$ are susceptible to either measles, mumps or rubella, ${ }^{38}$ and immigrants from tropical countries are 5-10 times more susceptible to varicella, ${ }^{39}$ which has serious implications for adult immigrants.

A large proportion $(20 \%-80 \%)$ of the immigrants who come from countries where chronic hepatitis B virus infection is prevalent are not immune and have not been immunized. In addition, immigrants are more likely to be exposed to hepatitis $\mathrm{B}$ virus ${ }^{40}$ in their households and during travel to countries where hepatitis B is prevalent. Immigrants from countries where chronic hepatitis B virus infection is prevalent (affecting $2 \%$ or more of the population) can benefit from screening and treatment to prevent hepatitis and hepatocellular carcinoma.

Foreign-born people account for $65 \%$ of all active tuberculosis in Canada, ${ }^{41}$ and screening and treatment for latent tuberculosis remain priorities for immigrants from countries in subSaharan Africa, Asia, and Central and South America. ${ }^{42}$ To promote patients' safety and adherence to therapy, patients must be informed of the risks and benefits of treatment in a culturally and linguistically appropriate manner. ${ }^{43,44}$ Refugees may already be aware of their HIV-positive status but may have limited knowledge of effective screening and treatment options. HIV-related stigma and discrimination ${ }^{45}$ put immigrants and refugees at risk for delayed diagnosis and unequal treatment rates for HIV infection. Immigrants are an unrecognized risk group for chronic hepatitis $\mathrm{C}$ virus infection and would benefit from early detection and appropriately timed treatment. $^{46}$

Subclinical strongyloidiasis and schistosomiasis can persist for decades after immigration and, if left untreated, can lead to serious morbidity or death through disseminated disease. ${ }^{47}$ 
Serologic tests for these intestinal parasites, rather than traditional stool testing, are recommended. ${ }^{48}$ Malaria is one of the leading causes of death worldwide, ${ }^{49}$ and delay in diagnosis and treatment of Plasmodium falciparum may lead to severe disease and even death. Migrant children are especially at risk for malaria and its complications. ${ }^{50}$

Recommendations for infectious diseases are summarized in Box 1B.

\section{Mental health and physical and emotional maltreatment}

Mental health care of immigrants has emerged as one of the most challenging areas for clinicians. ${ }^{15}$ Among refugees, depression commonly co-occurs with post-traumatic stress disorder and other anxiety disorders, ${ }^{51}$ which can complicate its detection and treatment. ${ }^{28}$ Conducting a systematic clinical assessment, or using a validated questionnaire in a language in which the patient is fluent, ${ }^{52}$ is recommended if the clinician practises in an integrated system that links patients with suspected depression to treatment programs with a stepped-care approach. Effective detection and treatment may also require the use of professional interpreters or trained culture brokers (not children or other family members) to identify patients' concerns, explain illness beliefs, monitor progress, ensure adherence, and address the social causes and the consequences of depression. ${ }^{28}$ The majority of those who experience traumatic events will heal spontaneously after reaching safety. ${ }^{53,54}$ Empathy, reassurance and advocacy are key clinical elements of the recovery process. Pushing for disclosure of traumatic events could cause more harm than good.

The children of ethnic minorities, including some recently settled immigrants and/or refugees, are disproportionately over-screened (up to 8.75 times more likely) and overreported as positive (up to four times more likely) for child maltreatment. ${ }^{55}$ False-positive reports could result in harm, leading to psychological distress, inappropriate family separation, impaired clinician-patient rapport and legal ramifications associated with the involvement of child protection services. ${ }^{56}$ Routine screening is not recommended; rather, clinicians should remain alert for maltreatment, either intimate partner violence or child maltreatment.

Recommendations related to mental health and maltreatment, both physical and emotional, are summarized in Box 1C.

\section{Box 1B: Summary of evidence-based recommendations for infectious diseases*}

\section{Measles, mumps and rubella}

Vaccinate all adult immigrants without immunization records using one dose of measles-mumps-rubella vaccine.

Vaccinate all immigrant children with missing or uncertain vaccination records using age-appropriate vaccination for measles, mumps and rubella.

\section{Diphtheria, pertussis, tetanus and polio}

Vaccinate all adult immigrants without immunization records using a primary series of tetanus, diphtheria and inactivated polio vaccine (three doses), the first of which should include acellular pertussis vaccine.

Vaccinate all immigrant children with missing or uncertain vaccination records using age-appropriate vaccination for diphtheria, pertussis, tetanus and polio.

\section{Varicella}

Vaccinate all immigrant children $<13$ years of age with varicella vaccine without prior serologic testing.

Screen all immigrants and refugees from tropical countries $\geq 13$ years of age for serum varicella antibodies, and vaccinate those found to be susceptible.

\section{Hepatitis B}

Screen adults and children from countries where the seroprevalence of chronic hepatitis B virus infection is moderate or high (i.e., $\geq 2 \%$ positive for hepatitis B surface antigen), such as Africa, Asia and Eastern Europe, for hepatitis B surface antigen, anti-hepatitis B core antibody and antihepatitis B surface antibody.

Refer to a specialist if positive for hepatitis B surface antigen (chronic infection).

Vaccinate those who are susceptible (negative for all three markers).

\section{Tuberculosis}

Screen children, adolescents $<20$ years of age and refugees between 20 and 50 years of age from countries with a high incidence of tuberculosis as soon as possible after their arrival in Canada with a tuberculin skin test.

If test results are positive, rule out active tuberculosis and then treat latent tuberculosis infection.

Carefully monitor for hepatotoxity when isoniazid is used.

\section{HIV}

Screen for HIV, with informed consent, all adolescents and adults from countries where HIV prevalence is greater than $1 \%$ (sub-Saharan Africa, parts of the Caribbean and Thailand).

Link HIV-positive individuals to HIV treatment programs and post-test counselling.

\section{Hepatitis C}

Screen for antibody to hepatitis $\mathrm{C}$ virus in all immigrants and refugees from regions with prevalence of disease $\geq 3 \%$ (this excludes South Asia, Western Europe, North America, Central America and South America).

Refer to a hepatologist if test result is positive.

\section{Intestinal parasites}

Strongyloides: Screen refugees newly arriving from Southeast Asia and Africa with serologic tests for Strongyloides, and treat, if positive, with ivermectin.

Schistosoma: Screen refugees newly arriving from Africa with serologic tests for Schistosoma, and treat, if positive, with praziquantel.

\section{Malaria}

Do not conduct routine screening for malaria.

Be alert for symptomatic malaria in migrants who have lived or travelled in malaria-endemic regions within the previous three months (suspect malaria if fever is present or person migrated from sub-Saharan Africa). Perform rapid diagnostic testing and thick and thin malaria smears.

*Order of listing considers clinical feasibility and quality of evidence. 


\section{Chronic and noncommunicable diseases}

People of certain ethnic backgrounds (specifically Latin Americans, Africans and South Asians) face a twofold to fourfold higher prevalence of type 2 diabetes mellitus than white people, ${ }^{57}$ with earlier onset and poorer outcomes. People with hypertension have the most to gain from treatment of obesity, high cholesterol, hypertension and hyperglycemia. Culturally appropriate diabetes education and lifestyle interventions are effective in preventing the disease or improving disease management. ${ }^{58}$ Iron deficiency is the most common nutritional deficiency in the world, ${ }^{59}$ and immigrant women ${ }^{60}$ and children can benefit from screening and supplementation.

Dental disease is often challenging for medical practitioners, but screening and treating pain with nonsteroidal antiinflammatory drugs can lead to better outcomes and more effective referrals for oral health care. ${ }^{61}$ In addition, there is value in recommending twice-daily tooth-brushing with fluor-

\section{Box 1C: Summary of evidence-based recommendations} for mental health and physical and emotional maltreatment*

\section{Depression}

If an integrated treatment program is available, screen adults for depression using a systematic clinical inquiry or validated patient health questionnaire (PHQ-9 or equivalent).

Individuals with major depression may present with somatic symptoms (pain, fatigue or other nonspecific symptoms).

Link suspected cases of depression with an integrated treatment program and case management or mental health care.

\section{Post-traumatic stress disorder}

Do not conduct routine screening for exposure to traumatic events, because pushing for disclosure of traumatic events in well-functioning individuals may result in more harm than good.

Be alert for signs and symptoms of post-traumatic stress disorder (unexplained somatic symptoms, sleep disorders or mental health disorders such as depression or panic disorder).

\section{Child maltreatment}

Do not conduct routine screening for child maltreatment. Be alert for signs and symptoms of child maltreatment during physical and mental examinations, and assess further when reasonable doubt exists or after patient disclosure.

A home visitation program encompassing the first two years of life should be offered to immigrant and refugee mothers living in high-risk conditions, including teenage motherhood, single parent status, social isolation, low socioeconomic status, or living with mental health or drug abuse problems.

\section{Intimate partner violence}

Do not conduct routine screening for intimate partner violence.

Be alert for potential signs and symptoms related to intimate partner violence, and assess further when reasonable doubt exists or after patient disclosure.

Note: PHQ-9 = nine-item Patient Health Questionnaire.

* Order of listing considers clinical feasibility and quality of evidence. idated toothpaste, as some immigrants may not be familiar with this approach to oral health. ${ }^{62}$ Loss of vision is the final common pathway for all eye diseases,${ }^{63}$ and all immigrants can benefit from having their visual acuity assessed soon after arrival in Canada.

Recommendations for chronic and noncommunicable diseases are summarized in Box 1D.

\section{Women's health}

To prevent unintended pregnancy, screening for unmet contraceptive needs should begin soon after a woman's arrival in Canada. Giving women their contraceptive method of choice (the intrauterine device being the most common contraceptive worldwide, although personal preferences vary), providing the contraceptive method on site and having a good interpersonal relationship all improve contraceptive-related outcomes. ${ }^{64}$

School vaccination programs vary by province, and immigrant girls and women may miss school programs for human papillomavirus vaccination, depending on their age at the time of arrival. Subgroups of immigrants, most notably South Asian and Southeast Asian women, have substantially lower rates of cervical cytology screening than Canadian-born women. ${ }^{65}$ Women who have never undergone cervical screening and those who have not had cervical screening in the previous five years account for $60 \%-90 \%$ of invasive cervical cancers. Providing information to patients, building rapport

Box 1D: Summary of evidence-based recommendations for chronic and noncommunicable diseases*

\section{Type 2 diabetes mellitus}

Screen immigrants and refugees $>35$ years of age from ethnic groups at high risk for type 2 diabetes (those from South Asia, Latin America and Africa) with fasting blood glucose.

\section{Iron-deficiency anemia}

\section{Women}

Screen immigrant and refugee women of reproductive age for iron-deficiency anemia (with hemoglobin).

If anemia is present, investigate and recommend iron supplementation if appropriate.

\section{Children}

Screen immigrant and refugee children aged one to four years for iron-deficiency anemia (with hemoglobin).

If anemia is present, investigate and recommend iron supplementation if appropriate.

\section{Dental disease}

Screen all immigrants for dental pain. Treat pain with nonsteroidal anti-inflammatory drugs and refer patients to a dentist.

Screen all immigrant children and adults for obvious dental caries and oral disease, and refer to a dentist or oral health specialist if necessary.

\section{Vision health}

Perform age-appropriate screening for visual impairment. If presenting vision $<6 / 12$ (with habitual correction in place), refer patients to an optometrist or ophthalmologist for comprehensive ophthalmic evaluation.

*Order of listing considers clinical feasibility and quality of evidence. 
and offering access to female practitioners can improve acceptance of Papanicolau (Pap) testing. ${ }^{66}$

Finally, newly arrived pregnant women are at increased risk for maternal morbidity. ${ }^{67}$ We identified social isolation, risks of unprotected or unregulated work environments, and sexual abuse (specifically in forced migrants) as priority areas for research.

Recommendations related to women's health are summarized in Box 1E.

\section{Knowledge translation}

We developed a summary of our recommendations and have engaged multiple stakeholders as partners to share these recommendations with their constituencies, including the Public Health Agency of Canada, Citizenship and Immigration Canada, regional health and public health authorities, immigrant community groups and primary care practitioners. These recommendations and their related evidence reviews are avail-

Box 1E: Summary of evidence-based recommendations for women's health*

\section{Contraception}

Screen immigrant women of reproductive age for unmet contraceptive needs soon after arrival to Canada.

Provide culturally sensitive, patient-centred contraceptive counselling (giving women their method of choice, having contraception on site and fostering a good interpersonal relationship).

Vaccination against human papillomavirus

Vaccinate 9- to 26-year-old female patients against human papillomavirus.

\section{Cervical cytology}

Screen sexually active women for cervical abnormalities by Papanicolaou (Pap) test.

Information, rapport and access to a female practitioner can improve uptake of screening and follow-up.

*Order of listing considers clinical feasibility and quality of evidence. able on the CMAJ website (see www.cmaj.ca/lookup/suppl /doi:10.1503/cmaj.090313/-/DC1). Forty primary care practitioners from across Canada with experience working with immigrants pilot-tested the recommendations, provided feedback on the presentation format and are helping to promote the guidelines through their networks. Finally, we sought feedback on our recommendations from our immigrant community partners (specifically, the Edmonton Multicultural Health Brokers Cooperative, which represents 16 ethnic communities) and continue to work with our community partners to improve access to health services.

\section{Directions for future research}

Immigrant populations are a heterogeneous group. Because of the selection processes that are in place, most immigrants arrive in good health, although some subgroups are at increased risk of chronic and infectious diseases and mental illness. More research is needed on strategies to address barriers to health services, most urgently for refugees, women and other immigrants with low income and language barriers. There is also a need to develop and study interventions for social isolation for pregnant immigrants and refugees. Data remain limited for immigrant children, refugee claimants and nonstatus persons and for many disease areas, including malaria morbidity, posttraumatic stress disorder and interventions for intimate partner violence.

More work must be done to improve immigrants' access to health services. We hope this evidence-based initiative will provide a foundation for improved preventive health care for immigrant populations.

For a summary of recommendations and clinical considerations, see Appendix 2, at www.cmaj.ca/lookup/suppl/doi:10.1503 /cmaj.090313/-/DC1.

Podcasts for practitioners and additional information for patients can be found at www.ccirhken.ca. 


\section{Selection of potentially preventable and treatable conditions}

Community-based primary health care practitioners see most of the immigrants and refugees who arrive in Canada. This is not only because Canada's health care system centres on primary care practice, but also because people with lower socioeconomic status, language barriers and less familiarity with the system are much less likely to receive specialist care. ${ }^{68}$

Guideline development can be costly in terms of time, resources and expertise. ${ }^{69}$ Setting priorities is critical, particularly when dealing with complex situations and limited resources. ${ }^{70}$ There is no standard algorithm on who should determine top priorities for guidelines or how this should be done, although burden of illness, feasibility and economic considerations are all important. ${ }^{71}$ Stakeholder engagement, to ensure relevance and acceptability, and the use of an explicit procedure for developing recommendations are critical in guideline development. ${ }^{72-74} \mathrm{We}$ chose primary care practitioners, particularly those who care for immigrants and refugees, to help the guideline committee in selecting conditions for clinical preventive guidelines for immigrants and refugees, with a focus on the first five years of settlement. A more detailed description of this Delphi process was published previously. ${ }^{15}$

\section{Methods}

We used a modified Delphi consensus process to select 20 high-priority conditions for guideline development. ${ }^{70,75,76}$ To begin, we identified key health conditions using an environmental scan, literature review and input from key informants from the Canadian Initiative to Optimize Preventive Care for Immigrants national network, a nascent network of immigrant health providers. This initial step identified 31 conditions. During the ranking process, survey participants were invited to list additional conditions. These conditions, if associated with potentially effective clinical preventive actions, were integrated into the pool of conditions for subsequent ranking.

We developed priority-setting criteria that emphasized inequities in health, building on a process developed for primary care guidelines affecting disabled adults. ${ }^{70,77}$ Importance or burden of illness is often used for setting priorities, usefulness or effectiveness is frequently used, and disparity is now a well-recognized component of many public health measures. ${ }^{78}$ We defined our criteria as importance, usefulness and disparity:

- Importance: Conditions that are the most prevalent health issues for newly arriving immigrants and refugees; conditions with a high burden of illness (e.g., morbidity and mortality).

- Usefulness: Conditions for which guidelines could be practically implemented and evaluated. Such guidelines refer to health problems that are easy to detect, for which the means of prevention and care are readily available and feasible, and for which health outcomes can be monitored.

- Disparity: Conditions that might not be currently addressed or that are poorly addressed by public health initiatives or illness-prevention measures that target the general population.

We (H.S., K.P., M.R., L.N.) purposively selected 45 primary care practitioners, including family physicians and nurse practitioners, recently or currently working in a setting serving recent immigrants and refugees. We sampled clinical settings from 14 urban centres across Canada to ensure in-depth experience with a variety of migrants. The settings also covered a range of health service funding models: community health centres (centres locaux de services communautaires in Quebec), refugee clinics, group and solo practices, and ethnic community practices. We aimed to select practitioners with substantial experience, academic expertise or local leadership roles who were willing to commit to offering future input into guideline development and dissemination.

Immigrant and refugee health is a new subdiscipline. The skills, knowledge and experience that define expertise have not yet been determined, and there are no examinations, certification or developed courses that can be used as a proxy for expertise. We believed that contextual knowledge, experience arising from engaged care of immigrants and refugees in Canada, and related work experience in international health were important factors in determining expertise. As a measure of expertise, we adapted a formula used by Médecins Sans Frontières. This criterion combines work with Médecins Sans Frontières in developed countries and in the field. Our criterion for experience was set at seven years or more and included all work in developing countries. It was calculated as number of years of experience with migrants in Canada $+(2 \times$ years of experience working in developing countries).

As prompts for decision-making, we asked our practitioner panel to make choices based on the defined criteria, imagining that the guidelines under development might be used at a clinic serving new immigrants or by physicians who do not often see immigrant and refugee patients. Just as clinical practice does, these criteria challenged practitioners to make choices based on competing demands.

This first round of the Delphi survey aimed to ensure that we had the appropriate health conditions under consideration and to begin developing some consensus as to priorities. Participants were asked to rank the 31 conditions identified initially and to propose conditions that were not on the initial list. We chose an a priori cut-off of $80 \%$ consensus for inclusion in the top 20. In the second round, we presented an unranked, modified version of this list, excluding all conditions that had already reached $80 \%$ consensus and adding newly proposed conditions. The remaining conditions to be included in the top 20 were determined by overall ranking in the second round. This list was reviewed by the codirectors of the Edmonton Multicultural Health Brokers Co-operative (www.mchb.org/OldWebsite2008/default.htm), a group representing over 16 ethnic communities that had initially requested preventive health guidance relevant for immigrant 
communities. In addition, the panel of experts who would be developing the guidelines reviewed the list. Then, during the final round, we requested approval, through a simple agree/disagree vote, of the process and the resulting list of priorities, with one-on-one interviews to resolve concerns in the two months following the ranking process.

Consent to participate in the Delphi survey was determined by completion of a questionnaire. Demographic questions elicited personal, professional and practice characteristics of the study participants. With each round, we sent to participants (by email) an explanation of the process to date, the prioritysetting criteria, instructions for filling out the survey and a link to the SurveyPro survey. Telephone follow-up was used to maximize response rate. We used Microsoft Excel for the analysis.

\section{Results}

Ninety per cent (40/45) of the selected practitioners agreed to participate. Four of the five participants who chose not to participate cited reasons of leave of absence or sabbatical leave, and the fifth cited workload. Ninety-five per cent of the consenting participants completed the first round of the survey, and $88 \%$ completed the second and third rounds (Figure 2A). The first two rounds of the Delphi consensus process took place between Mar. 5 and May 31, 2007.

The 40 participants consisted of 35 physicians and five nurse practitioners or nurses with expanded roles. Participants were predominantly women and had been in practice for an average of 14 years. They worked an average of 16 hours per week with immigrants and refugees. More than $80 \%$ spoke two or more languages (Table 2A).
The average length of experience working with refugees and immigrants in Canada was 7.5 years; $64 \%$ of participants had some experience working in developing countries, with a median overseas duration of 16 (range 1-120) months. Thirtyone per cent of primary care practitioners self-identified as being an immigrant or refugee; of the remainder, $38 \%$ selfidentified as being the child of an immigrant or refugee (of the 35 practitioners who responded to this optional question).

Forty-five per cent of participants identified themselves as having had prior training in the field, which included accredited tropical medicine courses, designated rotations during residency, work exposures before becoming a health care practitioner, and conferences or self-directed studies in multicultural or cross-cultural medicine.

The refugees and immigrants with whom most practitioners interacted came from all parts of the world; using an average of straight ranking ( 1 to 6 ) of regions, south and central Africa was estimated as the most frequent source region of immigrants for these practitioners. Children formed, on average, $30 \%$ of clientele, and women, $41 \%$. Seventy-one per cent of migrants were estimated to have been in Canada less than five years, and $73 \%$ were involuntary migrants. Involuntary migrants included refugee claimants, so-called Convention Refugees and internally displaced persons (although this is not really an issue for Canada).

Box 2A lists the top 20 conditions for which practitioners identified a current need for guidelines on the basis of our criteria. In the first round, $80 \%$ consensus was reached to include 11 conditions. Eighty per cent consensus was also reached to exclude three conditions from the process: Chagas disease, colon cancer and prostate cancer. Three well-defined and unique conditions were proposed for the second round of

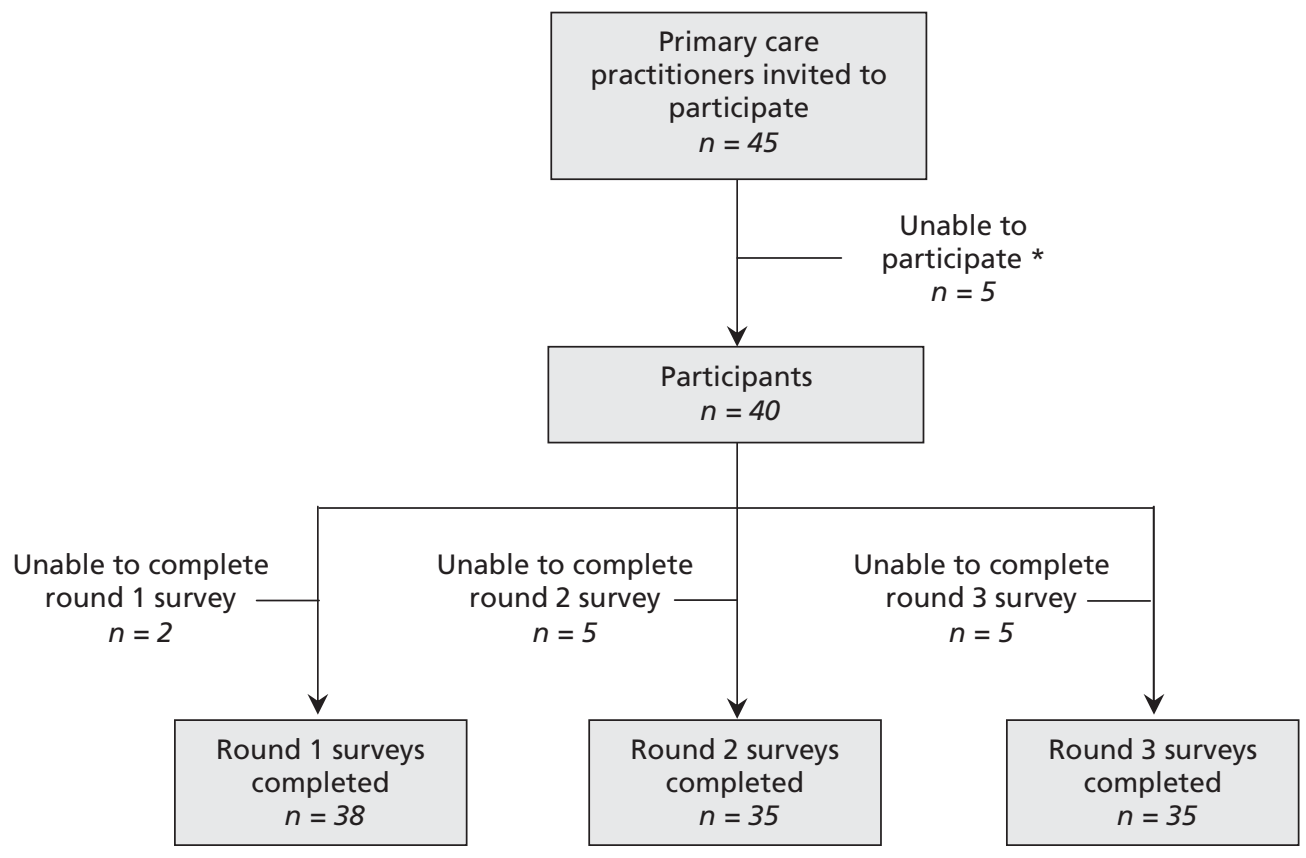

Figure 2A: Participant sampling and response rate. *One person was on sabbatical, three were on a leave of absence and one cited workload. Adapted, with permission, from Swinkels and associates. ${ }^{15}$ 
ranking: osteoporosis, contraception and vision screening. The nine conditions selected in the second round were based on average ranking (Box 2A).

The list of top 20 conditions was reviewed and approved, with one modification, by the panel of key experts who would be developing the guidelines: routine vaccine-preventable diseases were considered a single priority, with tetanus, diphtheria and polio combined with measles, mumps and rubella for the purposes of guideline development. As a final step, we sent the 20 identified conditions to survey participants for approval and discussion; all 35 people who participated in this round approved (i.e., $88 \%$ of the 40 original participants).

\section{Discussion}

Refugees and many immigrants may have poor or deteriorating health, because of conditions experienced before, during or after arrival to Canada. A health care system that is poorly adapted to their needs compounds this situation, resulting in further marginalization. Our Delphi consensus process used practitioners' years of field experience strategically to identify

Table 2A: Demographic characteristics of 40 participants in Delphi consensus process*

\begin{tabular}{|c|c|}
\hline Characteristic & $\begin{array}{l}\text { No. }(\%) \text { of } \\
\text { participants }\end{array}$ \\
\hline Sex, female $(n=40)$ & $25(62)$ \\
\hline Age, yr, mean & 42.5 \\
\hline Length of practice, yr, mean & 14.0 \\
\hline \multicolumn{2}{|l|}{ Province of practice $(n=40)$} \\
\hline British Columbia & $7(18)$ \\
\hline $\begin{array}{l}\text { Prairies (Alberta, Saskatchewan, } \\
\text { Manitoba) }\end{array}$ & $4(10)$ \\
\hline Ontario & $17(42)$ \\
\hline Quebec & $8(20)$ \\
\hline $\begin{array}{l}\text { Maritime (New Brunswick, Nova Scotia, } \\
\text { Prince Edward Island, Newfoundland } \\
\text { and Labrador) }\end{array}$ & $4(10)$ \\
\hline \multicolumn{2}{|l|}{ Type of practice $(n=39)$} \\
\hline Solo & $2(5)$ \\
\hline $\begin{array}{l}\text { Group (excluding those in a community } \\
\text { health centre) }\end{array}$ & $19(49)$ \\
\hline Community health centre & $18(46)$ \\
\hline \multicolumn{2}{|l|}{$\begin{array}{l}\text { Level of cross-cultural exposure and } \\
\text { expertise }\end{array}$} \\
\hline $\begin{array}{l}\text { Experience working with immigrants or } \\
\text { refugees, mean, yr }\end{array}$ & 7.5 \\
\hline $\begin{array}{l}\text { Medical experience in low- and middle- } \\
\text { income countries }(n=39)\end{array}$ & $25(64)$ \\
\hline $\begin{array}{l}\geq 7 \text { years' experience (criteria adapted } \\
\text { from Médecins Sans Frontières) }(n=40)\end{array}$ & $26(65)$ \\
\hline Bilingual $(n=40)$ & $33(82)$ \\
\hline Speaks more than 2 languages $(n=40)$ & $17(42)$ \\
\hline
\end{tabular}

preventable and often unrecognized clinical care gaps that can result from such majority-system biases.

An overarching goal of our guideline development project was to supplement guidelines that exist for the general Canadian population ${ }^{79}$ by focusing on health inequities. We therefore selected a high proportion of practitioners who work with refugees, a particularly vulnerable subgroup of immigrants prone to disparities. Using practitioners to select conditions ensured both that the needs of the future guideline-users were given priority and that conditions presenting serious clinical challenges, but that might be under-represented in the literature, were included. In working with perceived needs of practitioners, we risked a reporting bias: overemphasizing popular stereotypes (e.g., the importance of infectious diseases), underemphasizing unrecognized or emerging conditions (e.g., vitamin $\mathrm{D}$ deficiency) $)^{80}$ and loss of precision in terms of specific populations (e.g., our list does not fully reflect the greatest needs of children).$^{81}$ Also, by deliberately selecting participants who work with refugees, we risked falsely stereotyping the health status of all immigrants by overemphasizing refugee-specific conditions and, conversely, by underemphasizing common heath risks, such as hypertension, that affect all immigrants.

The Delphi process generated 20 conditions for guideline development that reflected the needs and priorities of primary care practitioners working with immigrants and refugees. Although immigrant screening has historically focused on infectious diseases, ${ }^{82}$ the conditions selected by survey participants extended across a spectrum of diseases, including infectious disease, dentistry, nutrition, chronic disease, maternal

\section{Box 2A: High-priority conditions}

1. Abuse and domestic violence*

2. Anxiety and adjustment disorder*

3. Cancer of the cervix

4. Contraception

5. Dental caries, periodontal diseases*

6. Depression*

7. Diabetes mellitus*

8. Hepatitis B*

9. Hepatitis $C$

10. HIVIAIDS*

11. Intestinal parasites*

12. Iron-deficiency anemia*

13. Malaria

14. Measles, mumps, rubella, diphtheria, tetanus, pertussis, polio and Hib disease

15. Pregnancy screening

16. Syphilis

17. Torture and post-traumatic stress disorder*

18. Tuberculosis*

19. Varicella (chicken pox)

20. Vision screening

*Conditions identified by consensus in first round (the rest were selected in the second round). 
and child health, and mental health. Mental health conditions were rated particularly high, and all four of the proposed mental health conditions reached $80 \%$ consensus in the first round of the Delphi survey. Four infectious diseases and three chronic diseases also reached $80 \%$ consensus. The inclusion of dental caries and periodontal disease in the top 11 conditions is notable, reflecting important cultural, as well as socioeconomic, barriers that refugees and immigrants face in access to dental care. ${ }^{83}$ This range of conditions suggests that immigrant and refugee medicine covers the full spectrum of primary care. Although infectious disease continues to be an important area of concern, we are now seeing mental health and chronic diseases as key considerations for recently arriving immigrants and refugees.

\section{Take-home messages}

Preventable and treatable, but often-neglected, health condi- tions were selected for the development of guidelines for immigrant populations made vulnerable because of health system bias. Criteria that emphasized addressing inequities in health helped in identifying gaps in clinical care. This evidence-based guideline initiative marks the evolution of immigrant and refugee medicine from a focus on infectious diseases to a more inclusive consideration of such chronic diseases as mental illness, dental disease, diabetes mellitus and cancer. We hope that this practitioner engagement process will improve the practicality of the evidence-based guidelines, help practitioners who already to work in the area to target and streamline their efforts, and encourage new practitioners to enter this challenging and interesting discipline.

For the complete description of the Delphi consensus process, see www.cmaj.ca/lookup/doi/10.1503/cmaj.090290. 


\section{Evaluation of evidence-based literature and formulation of recommendations}

A variety of methods are used for developing clinical guidelines and practice recommendations. ${ }^{84}$ We used the recently developed approach of moving away from recommendations classified by letters and numbers to the simplified classification system recommended by the Grading of Recommendations Assessment, Development and Evaluation (GRADE) Working Group ${ }^{85}$ and applied this to clinical preventive actions. Our guideline development process followed the Appraisal of Guidelines for Research and Evaluation (AGREE) instrument (www.agreetrust.org), which is recognized internationally as providing best-practice criteria for evidence-based guideline development.

We developed our recommendations on the basis of a prespecified process overseen by the guideline committee of the Canadian Collaboration on Immigrant and Refugee Health. Defining a methods process ensured that each guideline was developed in a systematic, reproducible manner and was based on the best evidence available. This process was based on existing guidelines, including the Canadian Medical Association (CMA) handbook on developing clinical practice guidelines $^{84}$ and the ADAPTE framework for adapting existing guidelines. ${ }^{86}$ Our process emphasized identifying immigrant- and refugee-specific evidence on efficacy and population characteristics from guidelines, systematic reviews and primary studies. When immigrant- and refugee-specific evidence was unavailable, we used specific criteria, adapted from the Cochrane Handbook, ${ }^{87}$ to judge how this evidence applied to our intended target population.

Conditions considered most important by practitioners caring for immigrants and refugees in Canada were assigned to groups of content experts, who were asked to develop evidence reviews with clinical conclusions for recent immigrants and refugees to Canada using a logic model and following a structured 14-step process. The guidelines focus on clinical care gaps ${ }^{84}$ during the "health settlement period," which we define as the first five years of residence in a new country for an immigrant or refugee. This is the period during which health practitioners are likely to have initial contact with this population and the time during which stressors from a person's country of origin and country of settlement are most likely to manifest. Immigrants and refugees are thus grouped together by this organizing period of resettlement; however, the heterogeneity, complexities and differences between and within these groups were recognized throughout the process.

In our process, we emphasized making clinically relevant recommendations and establishing an extension to existing guidelines rather than a replacement or revision.

\section{Methods}

We used the AGREE checklist to guide the overall development process: a panel of experts and a guideline committee set the scope and purpose of the guidelines, and stakeholders were engaged to select priority conditions and to merge recommendations. To ensure rigour and applicability, we developed 14 standardized steps (described below and summarized in Box 1A in section 1 of this article, above). The guideline committee and other guideline experts and practitioners provided feedback to improve clarity of presentation. We accepted funding only from university and government sources, to ensure editorial independence. Here we describe the steps in our standardized evidence review.

\section{Step 1: Develop clinician summary table}

A standardized clinician summary template was used in setting the framework for each selected condition. During subsequent steps, this clinician summary table was used to focus development of the preventive guidelines, on the basis of the condition's prevalence in the population of interest, population-specific clinical considerations (e.g., stigma and awareness of screening and treatment options), clinical actions upon migration, screening tests, screening interval or timing, and treatment.

\section{Step 2: Develop logic model and key questions}

Our logic model, which illustrates a plausible causal pathway for each guideline, was adapted from the US Preventive Services Task Force, ${ }^{88}$ with the addition of a box to consider patient perspectives (for an example, see Figure 3A). The logic model outlines the population of interest (immigrants and refugees); the intervention (i.e., screening); the target condition; adverse effects of screening, diagnosis and treatment; treatment options and outcomes; and the link between treatment and reductions in morbidity and mortality. The model illustrates how identification of the condition can be expected to lead to treatment and reduced morbidity and mortality in the population of interest. This logic model identified the need to consider whether intermediate outcomes would be accepted as the basis for the recommendations, and if so, the strength of association between intermediate and clinical outcomes. For example, high-risk behaviour is an intermediate outcome in reducing morbidity and mortality from HIV.

Review group leaders were asked to use this logic model to define the PICO (population, intervention, comparison and outcome) format for each clinical action. These elements guided the search for evidence.

\section{Step 3: Set the stage for admissible evidence}

We followed the process used by the US Preventive Services Task Force and the Canadian Task Force on Preventive Health Care to focus on evidence most critical to making a recommendation. ${ }^{84}$ We began with searches of specific guidelines and systematic reviews for the target population of immigrants and refugees, to document the current state of direct evidence. We extended these searches to capture evidence from the general population. The search strategy was modelled on that used by the Cochrane Collaboration ${ }^{89}$ and 
was conducted by one of two clinical librarians. The following databases were searched: MEDLINE, PreMEDLINE, Cochrane Database of Systematic Reviews, Database of Abstracts of Reviews of Effectiveness, Embase, CINAHL, National Guideline Clearing House and the CMA Infobase. We also searched the databases and publications of the Canadian Task Force on Preventive Health Care, the US Preventive Services Task Force, the Centers for Disease Control and Prevention, and the World Health Organization. We asked authors to create flow charts of their searches, using the Preferred Reporting Items for Systematic Reviews and Meta-analyses (PRISMA) ${ }^{90}$ framework as a template.

\section{Step 4: Assess eligibility of systematic reviews}

Two members of the review group independently reviewed the search strategies, abstracts and relevant full-text articles on the basis of the inclusion criteria and specified outcomes of interest.

Data from each eligible systematic review were extracted and documented in a table with the following headings: author and year, objective, number and types of studies included, setting, participants, intervention and findings. If no eligible systematic review was found, then the review group team searched for the next best available study (randomized controlled trials, observational studies) that addressed the question.

\section{Step 5: Search for data specific to immigrant and refugee populations}

A tailored search process was used to gather information on population-specific considerations relevant to immigrants and refugees in the following areas:

- baseline risk (prevalence) versus the Canadian general population

- rate of clinically important beneficial and harmful outcomes (e.g., mortality, morbidity)

- genetic and cultural factors (e.g., knowledge, attitudes, practices, cultural preferences, dietary preferences)

- compliance variation (e.g., physicians' and patients' adherence to recommendations)

\section{Step 6: Refocus on key clinical preventive actions and key questions}

After reviewing the literature and available evidence, review group teams were asked to focus on the most relevant clinical action(s) and immigrant and refugee subpopulation(s) and to select three or fewer candidate recommendations with added value over and above existing guidelines.

\section{Step 7: Assess quality of systematic reviews}

For each recommendation, all relevant systematic reviews were compared to ensure consistency among findings. If the conclusions of the systematic reviews were consistent, the most recent review was selected. Any inconsistencies in reviews were explicitly addressed: reasons for inconsistencies, including the evidence base or the interpretation, were explored, and the most appropriate systematic review was selected, considering the purposes of these guidelines.

The most relevant systematic reviews were then assessed

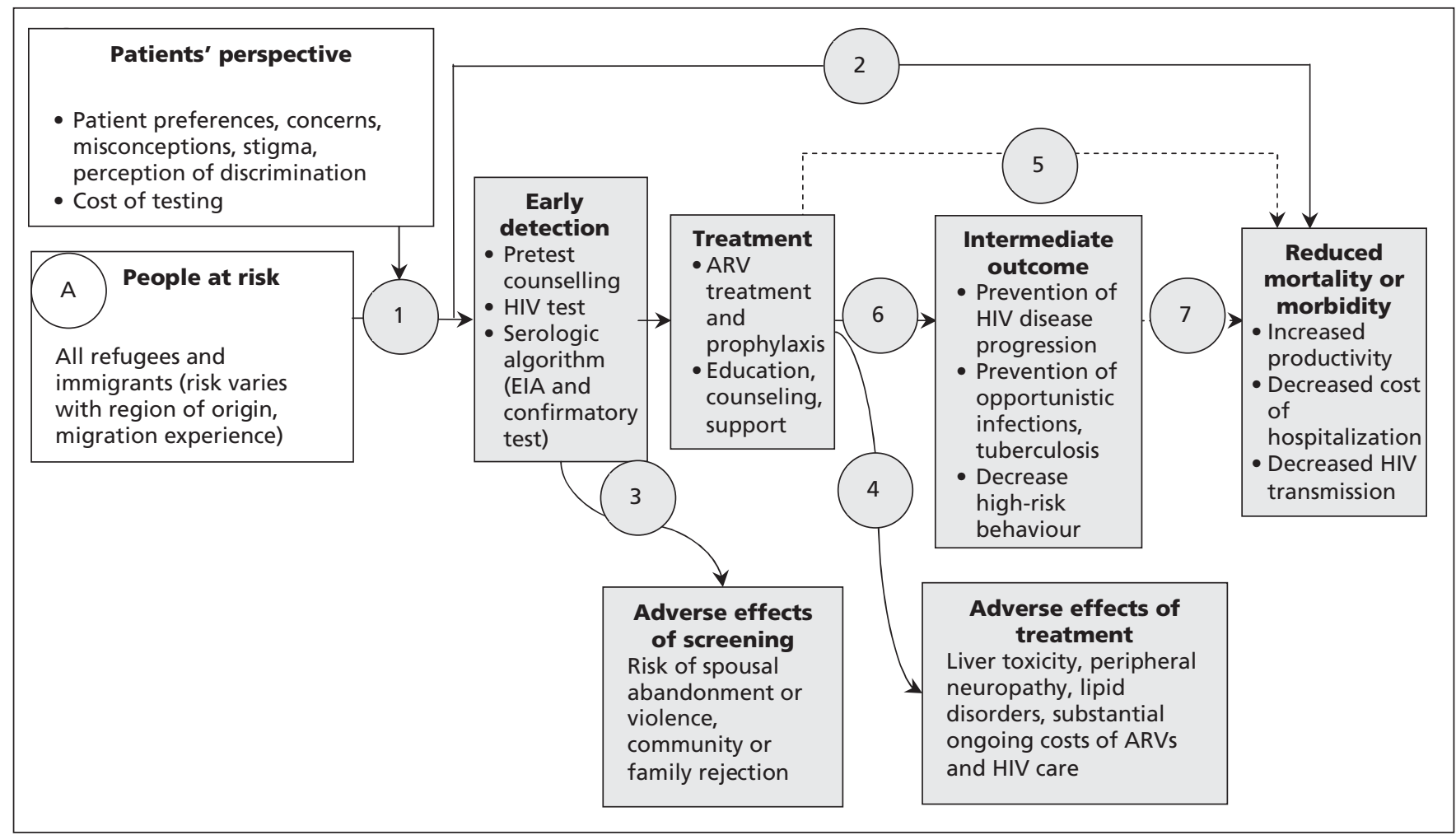

Figure 3A: Sample logic model for HIV (adapted from US Preventive Services Task Force). ${ }^{88}$ Open rectangles designate the potential screening population and patient factors to be considered; shaded rectangles designate interventions and related outcomes; and circles and numbers provide points in the evidence chain that were used to develop the search questions. Note: ARV = antiretroviral, EIA = enzyme immunoassay. 
for quality to ensure they met the four criteria assessed in the National Institute for Health and Clinical Excellence (formerly the Health Development Agency) critical appraisal tool for evidence-based briefings or reviews of reviews: ${ }^{91}$ systematicity (the review must apply a consistent and comprehensive approach), transparency (the review must be clear about the processes involved), quality (the review must have appropriate methods and analysis) and relevance (the review must be relevant in terms of focus; i.e., populations, interventions, outcomes and settings).

\section{Step 8: Search for evidence to update selected systematic reviews}

To find new primary studies published since the selected systematic review, a search was conducted using the same approach as in step 3 .

\section{Step 9: Assess eligibility of new studies}

As in step 4, two reviewers independently screened for relevant studies and then assessed each study for eligibility. Each relevant study was summarized to describe study design, the clinical intervention, details about length of intervention and follow-up, outcomes, population characteristics and data analysis.

For studies evaluating the effectiveness or safety of treatment or screening, the Cochrane Effective Practice and Organisation of Care Review Group's data collection checklist ${ }^{92}$ and the Newcastle-Ottawa Scale ${ }^{93}$ for assessing the quality of nonrandomized studies in meta-analyses were used to assess study limitations.

\section{Step 10: Integrate data from updated search}

Any new relevant and eligible studies that could modify or substantially strengthen the conclusions of the "reference" systematic review were assessed and added to the worksheet.

\section{Step 11: Synthesize final evidence bank and draft two key clinical actions}

The review group teams synthesized the evidence from the updated systematic reviews, explicitly incorporating clinical considerations and value judgments specific to immigrant and refugee populations to draft preferably no more than two key clinical actions, targeting (where necessary) specific populations or regions.

Box 3A: Grades of evidence of the Grading of

Recommendations Assessment, Development and Evaluation (GRADE) Working Group

(www.gradeworkinggroup.org)

- High quality: Further research is very unlikely to change our confidence in the estimate of effect.

- Moderate quality: Further research is likely to have an important impact on our confidence in the estimate of effect and may change the estimate.

- Low quality: Further research is very likely to have an important impact on our confidence in the estimate of effect and is likely to change the estimate.

- Very low quality: We are very uncertain about the estimate.

\section{Step 12: Develop table for summary of findings}

Both desirable and undesirable effects of the intervention were summarized, in both absolute and relative terms, for each patient-important outcome using the summary-of-findings table format adopted by the Cochrane Collaboration..$^{94}$ The quality for each outcome was assessed using the items specified by the GRADE Working Group (indirectness, consistency, precision, reporting bias and study limitations) (Box 3A). Observational studies that met these five criteria were upgraded if they also met one of three additional criteria (dose-response, influence of confounding variables, large effect). ${ }^{85}$ A separate table was developed for each clinical action or question. For dichotomous outcomes, relative risks or odds ratios were extracted from the reference systematic review (or next best available study). Number needed to treat for one person to benefit was calculated as $1 /($ control event rate $\times[1-$ relative risk $])$. The control event rate was taken from the control group of the reference systematic review or best available study.

\section{Step 13: Identify gaps in evidence and needs for future research}

The review group teams identified gaps in the literature and outlined recommendations for future research on such topics as implementation, inequalities and vulnerable groups, costeffectiveness and implications of applying the recommendations in health care settings.

\section{Step 14: Develop clinical preventive recommendations}

For each condition, the guideline committee reviewed the clinician summary table, the logic model and the summaryof-findings tables and met with the review group leader to clarify details. Then, for each key clinical action, the guideline committee discussed each of the issues in the GRADE system (see Table $1 \mathrm{~B}$ in section 1 of this article, above): ${ }^{16-18}$ the balance between desirable and undesirable effects (the relative importance of burden, benefits and harms), quality of the available evidence, and values and preferences. We explicitly decided not to use cost and feasibility in judging the basis of the recommendation because we did not have sufficient confidence in the data. Rather than report the strength of the recommendation as weak or strong, the guideline committee chose to make the recommendation only in the event of net benefits and to report the basis for the recommendation, to provide clinicians with key information to consider when selecting or discussing the preventive recommendation with a patient. The guideline committee took votes if the agreement was not unanimous, and the majority prevailed.

\section{Discussion}

This 14-step process was useful for ensuring sufficient uniformity among the transdisciplinary teams for each condition. Specifically, this systematic approach enabled the review group teams to meet the requirements of the GRADE qualityassessment process and the steering group to apply the GRADE recommendation process. These steps were also designed to conform with AGREE, the current quality stan- 
dard for guidelines. We worked with each review group leader and team to ensure we met the 23 AGREE criteria in six domains: scope and purpose, stakeholder involvement, rigour of development, clarity and presentation, applicability and editorial independence. ${ }^{16}$

\section{Take-home messages}

We combined the AGREE best-practice framework, the current quality standard for guidelines, with the recently developed GRADE approach to quality assessment to develop evidence-based clinical preventive guidelines for immigrants and refugees to Canada. Here, we have documented the systematic approach used to produce the evi- dence reviews and apply the GRADE approach. The 14-step approach included building on evidence from previous systematic reviews, searching for and comparing evidence between general and specific immigrant populations, and applying the GRADE criteria for making recommendations. The basis of each recommendation (balance of benefit and harm, quality of evidence, values) is stated explicitly to ensure transparency.

For a more complete description of the evaluation of the literature and formulation of recommendations, see www.cmaj.ca/lookup /doi/10.1503/cmaj.090289. 


\section{Measles, mumps, rubella, diphtheria, pertussis, tetanus and polio}

Vaccination is one of the most beneficial and cost-effective measures for preventing disease. ${ }^{95,96}$ Before routine vaccination, the annual burden of measles, mumps, rubella, diphtheria, pertussis, tetanus and polio in the United States and Canada was considerable. The incidence of and mortality from these diseases have been reduced sustantially: by more than $99 \%$ for measles, rubella, diphtheria and polio, by more than $95 \%$ for mumps, and by more than $92 \%$ for pertussis and tetanus relative to annual morbidity and mortality before introduction of the corresponding vaccines. ${ }^{97}$ Despite these successes, the recent outbreaks of pertussis in California, outbreaks of mumps in the United States and Canada in 2005-2006 and the ongoing transmission of polio in the past five years, with recent spread to Tajikistan, highlight the need to maintain high levels of herd immunity and to identify and vaccinate susceptible groups so that outbreaks can be prevented. ${ }^{98,99}$ Almost $20 \%$ of the Canadian population is foreign born, ${ }^{100}$ and in the past 30 years the majority of these people (more than 70\%) have originated from countries where vaccination coverage may be suboptimal or where several of the childhood vaccines that are routine in Canada are not part of the national vaccination schedule. ${ }^{101}$ Immigrants are therefore likely to be an unrecognized group at risk for childhood vaccinepreventable diseases. We conducted an evidence review to guide primary care practitioners in the need to assess and update childhood vaccination in the immigrant population. The recommendations of the Canadian Collaboration for Immigrant and Refugee Health on updating vaccines are outlined in Box 4A.

\section{Methods}

We used the 14-step method developed by the Canadian Collaboration for Immigrant and Refugee Health ${ }^{16}$ (summarized in section 3 of this article, above). We considered the epidemiology of measles, mumps, rubella, diphtheria, pertussis, tetanus and polio in immigrant populations and defined clinical preventive actions (interventions), outcomes and key clinical questions. We searched MEDLINE (Ovid), MEDLINE InProcess, Embase, CINAHL and the Cochrane Library from Jan. 1, 1950, to Jan. 14, 2010, for studies pertinent to immigrants and from Jan. 1, 1997, to Jan. 14, 2010, for studies pertinent to the general population. Detailed methods, search terms, case studies and clinical considerations can be found in the complete evidence review for this topic (Appendix 3, available at www.cmaj.ca/lookup/suppl/doi:10 .1503/cmaj.090313/-/DC1).

\section{Results}

In the search for systematic reviews and guidelines for immigrants regarding measles, mumps, rubella, diphtheria, pertus- sis, tetanus and polio, we identified and screened 242 records, of which none met the eligibility criteria. In the search for systematic reviews and guidelines involving these diseases in the general population, we identified 6293 articles, of which 24 met the eligibility criteria. A search for articles reporting information about admission to hospital and mortality associated with measles, mumps, rubella, diphtheria, pertussis, tetanus and polio identified 3888 articles (after duplicates were removed), of which 59 were relevant, and one of these was critical for this review ${ }^{97}$ (Table 4A). In addition, a search for articles about immigrants and measles, mumps, rubella, diphtheria, pertussis, tetanus and polio identified 1177 articles (duplicates removed), of which 54 were relevant, addressing the following areas: epidemiology, prevaccination screening, knowledge and compliance, treatment and vaccination in the immigrant population.

\section{What is the burden of vaccine-preventable disesases in immigrant populations?}

A large proportion of immigrants and refugees, particularly adults, are likely to be susceptible to vaccine-preventable diseases because of underimmunization, waning immunity or both. Underimmunization likely plays an important role, given that vaccine coverage globally ranges from $50 \%$ to $90 \%$, that routine childhood vaccination began only in the mid-1970s and that rubella and mumps vaccines are not administered routinely in most developing countries. ${ }^{101}$ Given the progress in global vaccination coverage, immigrant children and adolescents are more likely than their parents to have received vaccines that are part of the World Health Organization Extended Program on Immunization (measles, diphtheria, pertussis, tetanus, polio, bacille Calmette-Guérin), but many may not have received other vaccinations that are part of the routine childhood vaccination program in Canada (mumps, rubella, varicella, Hemophilus influenza, Streptococcus pneumoniae). Many immigrants, especially adults, do not have vaccination records, and even when present, more than $50 \%$ of such records may not be current according to the host country's vaccination schedules. ${ }^{38,102}$ Seroprevalence studies consistently have shown that a large proportion of adult immigrants are susceptible to rubella (about $80 \%-85 \%$ immune but as low as $75 \%$ ) and tetanus (about 50\%-60\% immune among those 20-30 years of age, but decreasing with increasing age). ${ }^{37,38,103,104} \mathrm{~A}$ higherthan-expected proportion of immigrants are involved in rubella outbreaks, and most reported cases of congenital rubella syndrome and neonatal tetanus have occurred in children born to unimmunized foreign-born mothers. ${ }^{105,106}$ In adult immigrants, seroprevalence studies of measles (> 95\% immune) and mumps (80\%-92\% immune but as low as $70 \%$ ) have generally shown adequate antibody levels, with 
some exceptions. ${ }^{38,107}$ However, immigrants have not been over-represented in recent measles and mumps outbreaks. ${ }^{98,108}$ Diphtheria seroprevalence in immigrants is low (range 35\%-50\%) and generally decreases with age..$^{37,104}$ To maintain herd immunity in the population, certain threshold levels of antibodies need to be maintained: $91 \%-94 \%$ for measles, $90 \%-92 \%$ for mumps, $83 \%-85 \%$ for rubella, $80 \%-$ $85 \%$ for diphtheria, $80 \%-85 \%$ for polio and $90 \%-94 \%$ for pertussis. ${ }^{109,110}$ Immigrants likely fall below this threshold for rubella and diphtheria, and a large proportion are also susceptible to tetanus and at risk for the morbidity and mortality associated with this disease..$^{37,38,103,104}$ Any population in which a large proportion of individuals are susceptible to vaccinepreventable disease will be at risk for disease transmission. High-risk groups must therefore be identified so that targeted vaccination programs can be formulated and implemented.

\section{Does vaccination against specific vaccine- preventable diseases decrease associated morbidity and mortality?}

\section{Relative benefits and harms of vaccination}

In the prevaccination era, diseases such as measles, mumps, rubella, diphtheria, pertussis, tetanus, smallpox and polio were very frequent and were a major cause of morbidity and mortality. These diseases also had enormous societal and economic costs, including time off school and work, physician visits and admissions to hospital. Childhood vaccination programs have decreased the morbidity from these diseases by more than $92 \%-99 \%$ and mortality by more than $99 \% .{ }^{97}$ Childhood vaccination programs have repeatedly been found to be one of the most cost-effective medical interventions. ${ }^{96}$

\section{Measles, mumps and rubella}

Measle-mumps-rubella vaccine is highly effective against measles and rubella. Almost 100\% of individuals are protected against measles after two doses, and more than $95 \%$ are protected against rubella after a single dose, with antibodies persisting for at least 15 years..$^{95,111}$ The effectiveness of mumps vaccine is lower and depends on the vaccine strain used, the time since vaccination and possibly the genotype of the wild type. ${ }^{12}$ In the recent US outbreaks, the effectiveness of mumps vaccine was estimated to be as low as $64 \%$ after one dose and $79 \%$ after two doses (Jeryl Lynn strain). ${ }^{112}$ Measle-mumps-rubella vaccine has been associated with fever (about $5 \%$ ), febrile convulsions $(0.3 \%)$, benign thrombocytopenia purpura $(<0.01 \%)$, parotitis (rarely) and arthritis (up to $25 \%$ in postpubertal women), usually within two weeks of vaccination. ${ }^{111,113,114}$ The frequency of adverse reactions in seronegative women, however, is higher among those who have never been vaccinated than among revaccinated seronegative women. ${ }^{111}$ In 1998 Wakefield and colleagues ${ }^{115}$ published a case series of 12 children with development disorders and

\section{Box 4A: Recommendations from the Canadian Collaboration for Immigrant and Refugee Health: vaccine-preventable} diseases

\section{Measles, mumps and rubella}

Vaccinate all adult immigrants without immunization records using one dose of measles-mumps-rubella vaccine.

Vaccinate all immigrant children with missing or uncertain vaccination records using age-appropriate vaccination for measles, mumps and rubella.

\section{Basis of recommendations}

\section{Balance of benefits and harms}

Childhood vaccination programs have dramatically decreased the incidence of and associated mortality from measles, mumps, rubella and congenital rubella (absolute difference of $95.9 \%-99.9 \%$ in reduction of cases and $100 \%$ in reduction of deaths). Serious adverse events, including autism (relative risk $0.92,95 \%$ confidence interval $0.68-1.24$ ), are not significantly associated with measles-mumps-rubella vaccine. Mumps and rubella are not part of routine vaccination programs in most source countries of origin for the majority of new immigrants. A large proportion of adult immigrants may be susceptible to rubella $(20 \%-30 \%)$ and at risk for having a child with congenital rubella syndrome.

\section{Quality of evidence}

High

\section{Values and preferences}

The committee attributed more value to preventing the risk of outbreaks and the individual burden due to these diseases and less value to the cost of vaccination.

\section{Diphtheria, pertussis, tetanus and polio}

Vaccinate all adult immigrants without immunization records using a primary series of diphtheria, tetanus and inactivated polio vaccine (three doses), the first of which should include acellular pertussis vaccine to also protect against pertussis.

Vaccinate all immigrant children with missing or uncertain vaccination records using age-appropriate vaccination for diphtheria, pertussis, tetanus and polio.

\section{Basis of recommendations}

\section{Balance of benefits and harms}

Childhood vaccination programs have dramatically decreased the incidence of and associated mortality from diphtheria, pertussis, tetanus and polio (absolute difference of $92.9 \%$ $99.9 \%$ in reduction of cases and $99.2 \%-100 \%$ in reduction of deaths) relative to the prevaccination period, without associated increases in serious adverse events. A large proportion of adult immigrants are susceptible to tetanus $(40 \%-50 \%)$ and diphtheria (about $60 \%)$, and the proportion susceptible increases for both with increasing age. To prevent individual morbidity and mortality and to prevent outbreaks, susceptible individuals must be identified and vaccinated.

\section{Quality of evidence}

High

\section{Values and preferences}

The committee attributed more value to preventing the risk of outbreaks and the individual burden due to these diseases and less value to the cost of vaccination. 
chronic gastrointestinal inflammation, which sparked widespread concern that measles-mumps-rubella vaccine was associated with autism. In 2010, The Lancet fully retracted this paper from the published record, after it became clear that several elements of the paper were incorrect. In addition, several subsequent studies have shown no association between measles-mumps-rubella vaccine and autism, including a population-based study of all Danish children born from 1991 to 1998 (> 500000 individuals) (relative risk $0.92,95 \%$ confidence interval 0.68-1.24). ${ }^{116}$

\section{Diphtheria, acellular pertussis, tetanus and polio}

Diphtheria and tetanus vaccines are highly effective, with protective levels of antibody developing in more than $95 \%$ of individuals after a primary series. Antibodies to diphtheria, which persist for up to 10 years, wane more rapidly than those for tetanus, which persist for up to 25 years. ${ }^{95,111}$ There is no immunologic correlate of protection for pertussis, but the clinical protective efficacy of the diphtheria, tetanus and acellular pertussis vaccine for children is about $85 \% .^{111}$ In over $99 \%$ of those vaccinated, protective levels of antibodies to all three serotypes of polio vaccine develop after three doses, and a one-time booster in adulthood is required to maintain immunity. For those who have received a primary series of tetanus and diphtheria vaccine, booster doses for tetanus-diphtheria vaccine (adult) are recommended every 10 years to maintain immunity. ${ }^{111}$ Individuals older than seven years of age who have not received a primary series should be given three doses of tetanus-diphtheria vaccine (adult), the first being tetanus-diphtheria-acellular pertussis, to pro- vide protection against pertussis. In addition, during management of any wound other than clean or minor wounds, these individuals should receive a primary series of vaccine and also tetanus immune globulin. ${ }^{111}$ Given that infants constitute the group at highest risk for development of severe pertussis, that adults are the main reservoir for pertussis and that antibodies wane over time (5-10 years after a primary series), all adults should be given a one-time booster of tetanus-diphtheriaacellular pertussis. ${ }^{111}$

Local pain, swelling and erythema are common after administration of the pediatric tetanus-diphtheria-acellular pertussis vaccine and are reported in up to $40 \%$ of those vaccinated; the corresponding rates are $75 \%$ after adult tetanusdiphtheria-acellular pertussis vaccine and 60\%-70\% after tetanus-diphtheria vaccine. Fever occurs in less than 5\% during a primary series; however, the pediatric and adult tetanusdiphtheria-acellular pertussis vaccines have not been associated with an increased risk of serious adverse events..$^{95,111,117}$

\section{Clinical considerations}

All adults and children without written immunization records should restart a primary immunization schedule appropriate for their age. ${ }^{111}$ An alternative, though somewhat less practical, approach is to test for antibodies to the major vaccine antigens and administer any vaccines to which the person has no immunity. A limitation to this approach is that serologic testing for diphtheria and tetanus is not widely available in most settings. Similarly, before one or two doses of trivalent measles-mumps-rubella vaccine are given, three serologic

Table 4A: Summary of findings for vaccination to prevent rubella and tetanus

Patient or population: General population

Setting: United States

Intervention: Vaccination, measles-mumps-rubella or diphtheria-tetanus

Comparison: Historical comparisons

Source: Roush SW, Murphy DG; Vaccine-Preventable Disease Table Working Group. Historical comparisons of morbidity and mortality for vaccine-preventable diseases in the United States. JAMA 2007;298:2155-63. ${ }^{97}$

\begin{tabular}{|c|c|c|c|c|c|}
\hline Outcome & \multicolumn{2}{|c|}{ Absolute effect } & Relative effect & $\begin{array}{l}\text { No. of participants } \\
\text { (studies) }\end{array}$ & $\begin{array}{l}\text { GRADE quality } \\
\text { of evidence }\end{array}$ \\
\hline \multicolumn{6}{|l|}{ Rubella } \\
\hline Cases, rubella & $\begin{array}{c}47745 \\
\text { (for } 1966-1968)\end{array}$ & $\begin{array}{c}47734 \text { fewer cases } \\
\text { (for 2006) }\end{array}$ & $\begin{array}{l}\text { Absolute difference: } \\
99.9 \% \text { reduction }\end{array}$ & NA (1) & $\mathrm{High}^{*}+$ \\
\hline Deaths & $\begin{array}{c}17 \\
\text { (for 1966-1968) }\end{array}$ & $\begin{array}{l}17 \text { fewer deaths } \\
\text { (for 2006) }\end{array}$ & $\begin{array}{l}\text { Absolute difference: } \\
100 \% \text { reduction }\end{array}$ & NA (1) & $\mathrm{High}^{*}+$ \\
\hline \multicolumn{6}{|l|}{ Tetanus } \\
\hline Cases & $\begin{array}{c}580 \\
\text { (for 1947-1949) }\end{array}$ & $\begin{array}{l}539 \text { fewer cases } \\
\text { (for 2006) }\end{array}$ & $\begin{array}{l}\text { Absolute difference: } \\
92.9 \% \text { reduction }\end{array}$ & NA (1) & $\mathrm{High}^{*}+$ \\
\hline
\end{tabular}

Note: GRADE = Grading of Recommendations Assessment, Development and Evaluation; NA = not applicable.

*Only one study.

tReduction in absolute numbers $>90 \%$. 
tests need to be done and the patient needs to return for the results before the vaccination series can be started. In one study, it was less costly and more effective to vaccinate all individuals if more than $80 \%$ had not completed the full diphtheria-tetanus vaccine series or if antibody seroprevalence to both diphtheria and tetanus was less than $51 \% .{ }^{118}$

Children are more likely than adults to have written immunization records; however, the optimal approach for those with vaccination records is challenging. Interpreting written records can be difficult because of language barriers and the fact that immunization schedules and products used in other countries may differ from those used in Canada. Even if records can be translated, they may not necessarily predict immunity. In several studies of internationally adopted children with seemingly appropriate records, many (up to 50\%) did not have serologic evidence of protective immunity to the specific antigen. This discordance has been ascribed to falsification of records, breaches in the cold chain in the countries of origin or host factors. ${ }^{119,120}$ Given this uncertainty, the most conservative approach would be to give a full vaccination series or, as mentioned above, perform prevaccination screening and vaccinate those without immunity. ${ }^{111}$

Barriers to vaccine uptake in children are well described, but a recent Canadian study showed that immigrant children were more likely to be vaccinated than children in the host population. ${ }^{121,122}$ In spite of this, many immigrant children may not have received all the vaccines that are part of the Canadian immunization schedule, and there are no structured catch-up vaccination programs for these children after arrival. Updating vaccination for adult immigrants is even more challenging than for children, in part because they are not a wellrecognized group at risk, but also because of the lack of routine well-adolescent or well-adult visits to access this population, the lack of structured programs to update vaccines and the cost (many vaccines are often not covered). In addition, missed opportunities for catch-up vaccination in adult immigrants when they come into contact with the health care system are well documented. ${ }^{123,124}$ With this in mind, health care providers need to remain vigilant in assessing and updating vaccinations in newly arrived immigrant and refugee children and adults.

\section{Recommendations of other groups}

The Canadian National Advisory Committee on Immunization recommends that all persons without written vaccination records should receive an age-appropriate primary vaccination series. Adults with no records or an unclear history of prior vaccination should receive a primary series of tetanusdiphtheria vaccine (three doses, one of which should be tetanus-diphtheria-acellular pertussis vaccine, to provide protection against pertussis), as well as a primary series for polio, as inactivated poliovirus vaccine (three doses, at baseline, 4-8 weeks and 6-12 months). The National Advisory Committee on Immunization also recommends that a single dose of measles-mumps-rubella vaccine be given to adults born after 1970 who do not have a history of measles or who are seronegative for mumps or rubella. ${ }^{111}$ Our recommendations highlight the importance of making primary care providers aware of the gaps in vaccination in newly arrived immigrants and refugees of all ages and the need to update vaccination against measles, mumps, rubella, diphtheria, pertussis and tetanus in this population.

\section{Take-home messages}

- A large proportion of immigrants are susceptible to several childhood vaccine-preventable diseases (especially rubella, diphtheria and tetanus) and are at risk for associated morbidity and mortality.

- Individuals susceptible to vaccine-preventable diseases must be identified and vaccinated to maintain herd immunity and prevent outbreaks.

- Health care providers need to aware of these gaps and ensure that they take all opportunities to update vaccinations in newly arrived immigrants and refugee children and adults.

For the complete evidence review for childhood vaccinepreventable diseases in immigrant populations, see Appendix 3 , available at www.cmaj.ca/lookup/suppl/doi:10.1503/cmaj .090313/-/DC1. 


\section{Varicella}

Varicella occurs at older ages in tropical countries, and most of these countries do not have varicella vaccination programs, which means that a large proportion of adolescent and adult immigrants from tropical countries (about 30\%) are susceptible to the disease. ${ }^{39,125-128}$ This is important because adults are more likely than children to contract severe varicella, with higher rates of pneumonia, encephalitis, admission to hospital and death. ${ }^{29}$ Varicella may also result in poor outcomes for pregnant women and their fetuses and infants. Several outbreaks of varicella have been documented in adult immigrants from tropical countries soon after arrival in temperate countries. ${ }^{130-134}$ In a recent US study, the mortality rate for varicella was higher in the foreign-born population than in the US-born population, likely because varicella occurs at older ages among immigrants. ${ }^{135}$ Canada has no programs to verify immune status to varicella, nor are there any systematic targeted or catch-up vaccination programs for the immigrant population. We conducted an evidence review to guide primary care practitioners in the need to assess varicella immune status in the immigrant population. The recommendations of the Canadian Collaboration for Immigrant and Refugee Health on varicella vaccination are outlined in Box 5A.

\section{Methods}

We used the 14-step method developed by the Canadian Collaboration for Immigrant and Refugee Health ${ }^{16}$ (summarized in section 3 of this article, above). We considered the epidemiology of varicella in immigrant populations and defined clinical preventive actions (interventions), outcomes and key clinical questions. We searched MEDLINE (Ovid), MEDLINE InProcess, Embase, CINAHL and the Cochrane Database of Systematic Reviews from 1950 to Jan. 19, 2011, for relevant articles pertinent to immigrants and from Jan. 1, 1997, to Jan. 19, 2011, for articles pertinent to the general population. Detailed methods, search terms, case studies and clinical considerations can be found in the complete evidence review for varicella vaccination (Appendix 4, available at www.cmaj.ca /lookup/suppl/doi:10.1503/cmaj.090313/-/DC1).

\section{Results}

In the search for systematic reviews and guidelines relevant to immigrants, we identified six records, but none of the studies followed a systematic review methodology. We identified 743 records for the general population, and of these, 11 were included: three recent guidelines from Canada and one guideline from the United States on preventing varicella, one review of varicella in pregnancy, two reviews on the efficacy of and adverse events due to varicella vaccine and the effectiveness of varicella vaccination programs, one review of the predictive value of a history of varicella infection, one systematic review on the cost-effectiveness of varicella vaccine, one review of barriers to adolescent vaccination and one systematic review on interventions to improve compliance with vaccination; we also identified a key article on the impact of varicella vaccination on health care utilization. ${ }^{136}$ In addition, the search for varicella AND immigrants or refugees identified 31 relevant articles that addressed epidemiology, costeffectiveness of vaccination and screening, vaccine knowledge and compliance. A search for articles dealing with varicella-associated morbidity and mortality identified 3400 articles, of which 122 were relevant. These included articles on epidemiology, admission to hospital and mortality due to varicella, screening for varicella immunity, vaccination effi-

\section{Box 5A: Recommendations from the Canadian Collaboration for Immigrant and Refugee Health: varicella (chicken pox)}

Ensure that immigrants and refugees of all ages are immune to varicella.

Vaccinate all immigrant children $<13$ years of age with varicella vaccine without prior serologic testing.

Screen all immigrants and refugees from tropical countries $\geq 13$ years of age for serum varicella antibodies, and vaccinate those found to be susceptible.

\section{Basis of recommendations}

\section{Balance of benefits and harms}

Varicella vaccination programs have substantially decreased ambulatory care visits (number needed to vaccinate [NNV] 794, 95\% confidence interval 688-990) and mortality (NNV 3031773 ) due to varicella in all age groups. The adverse effects of vaccination are minimal and include minor pain and redness at the injection site, rashes and fevers. A large proportion ( $>30 \%$ ) of adolescents and adult immigrants from tropical countries are susceptible to varicella because it occurs at an older age in tropical countries and because most of these countries do not have a varicella vaccination program. As a result, immigrants from tropical countries are at increased risk of developing severe varicella after arrival in Canada, as varicella develops at an older age and there are no systematic catch-up varicella vaccination programs for immigrants.

\section{Quality of evidence}

Moderate

\section{Values and preferences}

The committee attributed high value to reducing morbidity and mortality from varicella, which has a high burden of disease in adolescent and adult immigrant populations. For children $<13$ years of age, it is cost-saving to vaccinate all without prior serologic testing. In a cost-effectiveness analysis of adult immigrants and refugees, it was most cost-effective to vaccinate without prior screening with a seroprevalence of $<84 \%$ (children, most adolescents and some adults) and to serotest before vaccination when the seroprevalence was between $85 \%$ and $95 \%$ (some adolescents and most adults). If serologic testing results in extra costs or presents a barrier to completion of the vaccination series, vaccination without prior serologic testing should be offered. The most effective strategy will be one that is tailored to the vaccination setting and that balances the cost and anticipated uptake of the vaccine and the availability of, compliance with and costs of serologic testing. 
cacy and cost-effectiveness, and vaccine knowledge and compliance. The key article on varicella-associated mortality (used for Table 5A) was identified in this search. ${ }^{135}$

\section{What is the burden of varicella in immigrant populations?}

Larger proportions of adolescents and adults from tropical countries are susceptible to varicella (about 50\% at age 15 years and about $10 \%-15 \%$ from age 30 to 35 years) than is the case for Canadians (about $10 \%$ at age 15 years and less than $2 \%$ from age 30 to 35 years). This is because the mean age at which varicella develops is older in tropical countries (10-15 years) than in temperate or cold countries (4-5 years). In certain tropical regions (the Caribbean, Sri Lanka, Singapore, Indonesia and rural areas of other countries such as Pakistan and India), a large proportion of adults older than 35 years may still be susceptible to varicella. Immigrants from tropical countries have been involved in several varicella outbreaks and have higher mortality from varicella. ${ }^{13,132-135,137,138}$

The severity of varicella increases with age. Older age of infection increases the risk of varicella in pregnancy, which may result in poor outcomes for both the mother and the baby. Pregnant women are more likely than other adults to develop varicella pneumonia, to be admitted to hospital and to die from varicella. Congenital varicella syndrome is associated with a fetal case fatality rate of up to $50 \%$, and survivors may have congenital anomalies, including limb hypoplasia, microcephaly and dermatomal scarring. ${ }^{139}$ In addition, $20 \%$ of newborns born to women infected with varicella between five days before and three days after delivery will develop neonatal varicella, which carries a case fatality rate of $30 \%-$ $50 \% .^{140,141}$

\section{Does screening for immunity to varicella and vaccinating those who are susceptible decrease morbidity and mortality?}

\section{Diagnostic tests to screen for varicella antibodies}

The most widely available tests to detect antibodies to varicella zoster virus are enzyme immunoassays. These tests are less sensitive than the fluorescent antibody to membrane antibody test, but they have good specificity (> 95\%). Enzyme immunoassays are sufficiently sensitive to detect protective antibodies after natural infection with varicella zoster virus $(60 \%-92 \%)$ but are inadequately sensitive to detect protective antibodies resulting from vaccination. ${ }^{142}$ Given that the majority of adult immigrants at risk for varicella in Canada originate from countries without routine childhood vaccination programs, enzyme immunoassay is an acceptable method of screening for immunity to varicella in this population.

\section{History of prior varicella to determine varicella immune status}

The majority of adults (> 95\%) who report a history of varicella are immune to the virus. ${ }^{143}$ However, a positive history of varicella may less reliably predict protective varicella antibodies in immigrant populations where varicella seroprevalence is lower than expected for age..$^{39,144}$ In adult immigrant populations, it may be more prudent to perform serologic testing on all individuals before vaccination or to vaccinate empirically (if serologic testing is not available), to avoid the potential poor outcomes associated with varicella.

\section{Efficacy of varicella vaccination}

A single dose of varicella vaccine is $80 \%$ to $85 \%$ effective in preventing disease of any severity and is more than $95 \%$ effective in preventing severe varicella. ${ }^{145}$ Vaccine efficacy for adults has been estimated at about $80 \%$. Because of plateauing rates of varicella between 2003 and 2006 and ongoing outbreaks, despite a one-dose vaccination schedule, the United States recommended a universal two-dose childhood varicella vaccination program (first dose at $12-15$ months, second dose at four to six years) in 2006. ${ }^{146}$ The National Advisory Committee on Immunization of Canada has also recently recommended that two doses of varicella vaccine be given to all children under 13 years of age. ${ }^{147}$ Adverse events associated with the vaccine include pain and redness at the injection site $(22 \%-35 \%)$, rash (which is sometimes varicella-like; $1 \%-5 \%)$ and fever $(4 \%-7 \%)$. Secondary transmission has been documented from five vaccine recipients. ${ }^{148}$ Varicella vaccine is a live attenuated vaccine that should be avoided in immunosuppressed individuals. It can be given to HIV-infected individuals with mild to moderate symptoms and a CD4 count above $200 \times 10^{6} / \mathrm{L}$ or less than $15 \% .{ }^{149}$

\section{Effectiveness of varicella vaccination to decrease morbidity and mortality}

Varicella was a common childhood illness before implementation of widespread universal childhood vaccination programs in Canada (2005) and the United States (1995). These programs have resulted in a significant decrease in the number of cases and in the number of varicella-associated hospital admissions and deaths $(>75 \%)$ in all age groups, including adults (Table 5A) ${ }^{135,136,150} \mathrm{~A}$ recent study of the effect of varicella vaccine in Ontario showed decreases in varicellaassociated hospital admissions (by 53\%, 95\% confidence interval [CI] 48\%-58\%), emergency department visits (by $43 \%, 95 \%$ CI $41 \%-44 \%$ ) and physician visits (by $45 \%, 95 \%$ CI $44 \%-45 \%$ ) relative to the prevaccination period (19921998)..$^{150}$

\section{Cost-effectiveness of varicella vaccination}

Cost-effectiveness analyses of routine varicella vaccination of preschool-age children have demonstrated that the vaccine (one or two doses) is cost-saving from a societal point of view. ${ }^{151,152}$ Most cost-effectiveness studies support serologic testing before the vaccine is given to adolescents and adults. A recent cost-effectiveness analysis of strategies to prevent varicella in adult immigrants and refugees found that various vaccination strategies were cost-saving, relative to no intervention, at the following seroprevalence thresholds: vaccinate all without prior serologic testing (threshold $84 \%$; would include immigrant children under 13 years of age, many ado- 
lescents and some adults); provide serologic testing for all, and vaccinate those found to be susceptible (threshold $85 \%-$ $92 \%$; would include some adolescents and most adults); provide serologic testing only for those with a negative or unknown history of varicella (threshold 93\%-95\%) and vaccinate those found to be susceptible; and no intervention (threshold $>95 \%) .{ }^{128}$

\section{Clinical considerations}

\section{What are the potential implementation issues?}

We found no data specific to newly arrived immigrants or refugees. General factors associated with low uptake of varicella vaccine were similar to those for other vaccines (e.g., low socioeconomic status, low parental education, younger maternal age, lack of knowledge about the disease and vaccination, negative beliefs about and attitudes toward immunization, fear of adverse effects, lack of transport, inconvenient clinic hours), and cost was the most important barrier. ${ }^{122,153}$ Adolescents and adults face additional barriers to vaccination relative to children, including lack of awareness of the need for vaccination, lack of routine well-adolescent or well-adult visits and lack of coordinated vaccination programs for these populations. ${ }^{124,154}$ The two most important barriers to vaccination for adult immigrants are likely to be cost of the vaccine and lack of awareness among health care providers that adult immigrants are at increased risk for severe varicella. In certain settings, serologic testing may result in extra costs or may present a barrier to completion of the vaccination series, in which case vaccination without prior serologic testing should be offered.

The most effective interventions to improve uptake of vaccination in the general population were instituting reminder or recall systems, educating target populations and vaccine providers, and reducing out-of-pocket costs. ${ }^{155,156}$ The most effective interventions to improve vaccination coverage among adults are standing orders and expanding access to vaccination in nontraditional settings (e.g., schools, workplaces and social gathering places such as churches and sports clubs). ${ }^{154,156}$

Table 5A: Summary of findings for vaccination to prevent varicella and related morbidity and mortality

Patient or population: People up to 49 years of age; ${ }^{136}$ national death records ${ }^{135}$

Setting: MarketScan databases with enrollees from over 100 health insurance plans of about 40 large US employers, from 1994 to

$2002 ;^{136}$ "National Center for Health Statistics multiple cause-of-death mortality data for 1990 through 2001 "135

Intervention: Vaccination against varicella

Comparison: Prevaccine era

Sources: Zhou F, Harpaz R, Jumaan A, et al. Impact of varicella vaccination on health care utilization. JAMA 2005;294:797-802. ${ }^{136}$

Nguyen HQ, Jumaan AO, Seward JF. Decline in mortality due to varicella after implementation of varicella vaccination in the United States. N Engl J Med 2005;352:450-8. ${ }^{135}$

\begin{tabular}{|c|c|c|c|c|c|c|}
\hline Outcome & \multicolumn{2}{|c|}{ Absolute effect } & $\begin{array}{l}\text { Relative effect } \\
\qquad(95 \% \mathrm{Cl})\end{array}$ & $\begin{array}{l}\text { No. of } \\
\text { participants } \\
\text { (studies) }\end{array}$ & $\begin{array}{l}\text { GRADE quality } \\
\text { of evidence }\end{array}$ & $\begin{array}{l}\text { Comments } \\
(95 \% \mathrm{Cl})\end{array}$ \\
\hline $\begin{array}{l}\text { Admission to } \\
\text { hospital (primary } \\
\text { diagnosis of } \\
\text { varicella) }\end{array}$ & 2.3 per 100000 & $\begin{array}{c}2.0 \text { fewer } \\
\text { per } 100000\end{array}$ & $\begin{array}{c}\text { RR } 0.13 \\
(0.04-0.41)\end{array}$ & $\begin{array}{l}\text { Not reported* } \\
\text { (1) }\end{array}$ & Hight & $\begin{array}{c}\text { NNT } 49975 \\
(45384-73443)\end{array}$ \\
\hline $\begin{array}{l}\text { Ambulatory visits } \\
\text { (primary } \\
\text { diagnosis of } \\
\text { varicella) }\end{array}$ & 215 per 100000 & $\begin{array}{l}126 \text { fewer } \\
\text { per } 100000\end{array}$ & $\begin{array}{c}\text { RR } 0.41 \\
(0.32-0.53)\end{array}$ & $\begin{array}{l}\text { Not reported* } \\
\text { (1) }\end{array}$ & Moderateł & $\begin{array}{l}\text { NNT } 794 \\
(688-990)\end{array}$ \\
\hline \multicolumn{7}{|l|}{$\begin{array}{l}\text { Death from } \\
\text { varicella }\end{array}$} \\
\hline US-born & $\begin{array}{l}0.19 \text { per } \\
1000000 \S\end{array}$ & $\begin{array}{c}0.12 \text { fewer per } \\
10000009\end{array}$ & $\begin{array}{c}\text { RR } 0.37 \\
(0.15-0.88)\end{array}$ & $\begin{array}{l}\text { Not reported* } \\
\text { (1) }\end{array}$ & Moderate & $\begin{array}{l}\text { NNT } 8354219 \\
(6191950- \\
43859649)\end{array}$ \\
\hline
\end{tabular}

Note: $\mathrm{Cl}=$ confidence interval; GRADE = Grading of Recommendations Assessment, Development and Evaluation; NNT = number needed to treat; RR = risk ratio.

*Because of the nature of the original studies, these studies did not report the total number of participants.

tGraded up two levels to reflect very strong evidence of association $(R R<0.2)$.

$\neq$ Graded up one level to reflect strong evidence of association $(R R<0.5)$.

§Age-adjusted rate from Nguyen et al. ${ }^{135}$

ๆUnadjusted rate from Nguyen et al. ${ }^{135}$ 


\section{Recommendations of other groups}

The National Advisory Committee on Immunization in Canada recently recommended that all children between the ages of 12 months and 12 years should receive two doses of varicella vaccine as primary immunization. ${ }^{147}$ Persons 13 years of age and older should receive two doses of vaccine a minimum of six weeks apart. ${ }^{111}$ Certain groups at increased risk for varicella, such as immigrants from tropical countries, women of child-bearing age, household contacts of immunocompromised people, health care workers and adults who work in other occupations with increased exposure to varicella (teachers, daycare workers, etc.), should be considered for targeted varicella vaccination. ${ }^{111}$ Since 2007, the US Advisory Committee on Immunization Practice has recommended that both children and adults receive two doses of varicella vaccine. ${ }^{146}$ Our recommendations highlight the importance of varicella vaccination for susceptible immigrants.

\section{Take-home messages}

- Among immigrants from tropical countries, a large proportion of adolescents (up to 50\%) and adults (up to 10\%) are susceptible to varicella and are at increased risk of severe varicella.

- Pregnant immigrant women and their babies are at highest risk for complications of varicella.

- The mean age at which varicella develops varies for different world regions: it is acquired at an older mean age in tropical countries (15 years) than in temperate and cold countries (5 years).

- In certain tropical regions (the Caribbean, Sri Lanka, Singapore, Indonesia and rural areas of other countries such as Pakistan and India), the mean age of acquiring varicella may be even older, and a large proportion of adults older than 35 years may still be susceptible to the disease.

- Immigrants and refugees of all ages from tropical countries would benefit from having their varicella immune status verified and being offered varicella vaccine if found to be susceptible.

For the complete evidence review for varicella in immigrant populations, see Appendix 4, available at www.cmaj.ca/lookup /suppl/doi:10.1503/cmaj.090313/-/DC1. 


\section{Hepatitis B}

Hepatitis B virus infection is an important global health problem affecting 350 million people worldwide and leading to one million premature deaths from chronic liver disease and hepatocellular carcinoma annually. ${ }^{157}$ About $3 \%$ of immigrants to Canada but only $0.5 \%$ of Canadian-born people have chronic infection with hepatitis B virus. ${ }^{40,158}$ Most people with chronic infection are asymptomatic, and their disease goes undetected and untreated. This is likely why mortality from viral hepatitis and hepatocellular carcinoma among immigrants is two to four times higher than in the Canadian-born population. ${ }^{10}$

Over the past 10 years, several medications have become available that decrease viral replication and morbidity from chronic liver disease. Screening in high-risk populations and targeted treatment have therefore become important strategies to decrease the burden of chronic infection with hepatitis B virus. An effective vaccine (available in Canada since 1982) is an important tool to control transmission of hepatitis B. Despite the disparity in populations and the availability of a safe and effective vaccine, no programs in Canada systematically screen immigrants for chronic hepatitis B virus infection, nor are there systematic targeted or catch-up hepatitis B vaccination programs outside of the childhood vaccination program. ${ }^{159}$ We conducted a review to quantify the burden of chronic hepatitis B among immigrants, to search for evidence of the effectiveness of screening and vaccination programs, and to identify barriers or challenges to implementing such programs. The recommendations of the Canadian Collaboration for Immigrant and Refugee Health on screening for and vaccinating against hepatitis B virus are outlined in Box 6A.

\section{Methods}

We used the 14-step approach developed by the Canadian Collaboration for Immigrant and Refugee Health ${ }^{16}$ (summarized in section 3 of this article, above). We considered the epidemiology of disease in immigrant populations and defined clinical preventive actions (interventions), outcomes and key clinical questions. We searched MEDLINE, Embase, CINAHL, the Cochrane Library and other sources from Jan. 1, 1996, to Jan. 1, 2010. Detailed methods, search terms, case studies and clinical considerations can be found in the complete evidence review for hepatitis B (see Appendix 5, available at www.cmaj.ca/lookup/suppl/doi:10.1503 /cmaj.090313/-/DC1).

\section{Results}

In the search for systematic reviews and guidelines for immi-

Box 6A: Recommendations from the Canadian Collaboration for Immigrant and Refugee Health: hepatitis B virus

\section{Screening}

Screen adults and children from countries where the seroprevalence of chronic hepatitis B virus infection is moderate or high (i.e., $\geq 2 \%$ positive for hepatitis B surface antigen). Refer those found to have chronic infection for evaluation and assessment of the need for treatment, and screen risk groups for hepatocellular carcinoma. Lifelong monitoring is required.

\section{Basis of recommendations}

\section{Balance of benefits and harms}

Screening for and then treating advanced chronic hepatitis B virus infection reduces the development of progressive liver failure (number needed to treat 19,95\% confidence interval [Cl] 15-44). Screening for hepatocellular carcinoma (by ultrasonography and serologic testing for $\alpha$-fetoprotein every six months) in certain risk groups with chronic hepatitis B virus infection decreases the risk of death from hepatocellular carcinoma (number needed to screen 2058, 95\% Cl 14624412). Prevalence of chronic hepatitis $B$ virus infection is higher among immigrants and refugees than among North Americans (mean $4 \% \mathrm{v} .<0.5 \%$ ). Toxicity varies by treatment regimen, but most therapies are well tolerated.

\section{Quality of evidence}

Moderate

\section{Values and preferences}

The committee attributed more value to preventing progressive liver failure and death from hepatocellular carcinoma and less value to the burden of screening for and treatment of adverse effects.

\section{Vaccination}

Screen adults and children from countries where the seroprevalence of chronic hepatitis B virus infection is moderate or high (i.e., $\geq 2 \%$ positive for hepatitis B surface antigen) for prior immunity to hepatitis B virus (antihepatitis B core antibody, anti-hepatitis B surface antibody). Vaccinate those found to be susceptible (negative for all three markers [hepatitis B surface antigen, anti-hepatitis B core antibody and anti-hepatitis B surface antibody]).

\section{Basis of recommendation}

\section{Balance of benefits and harms}

Universal perinatal and childhood vaccination in countries where chronic hepatitis $B$ virus infection is endemic have dramatically reduced chronic infection with hepatitis $B$ virus (number needed to vaccinate $12,95 \% \mathrm{Cl} 11-12$ ) and decreased mortality from hepatocellular carcinoma (relative risk $0.725,95 \% \mathrm{Cl} 0.518-1.015) 15$ years after initiation of vaccination programs. In countries with low seroprevalence of chronic hepatitis B virus infection (i.e., $<2 \%$ positive for hepatitis B surface antigen), vaccination of adults decreases development of acute infection. Adverse reactions to vaccination are minor and self-limited.

\section{Quality of evidence}

Moderate

\section{Values and preferences}

The committee attributed more value to reducing transmission of hepatitis B virus infection, a potentially fatal disease, to close contacts than to the burden of screening and vaccination. 
grants, we identified and screened 54 records. Of the six that met our eligibility criteria, all were narrative reviews on screening immigrants for hepatitis B and were excluded because none followed a systematic review method. A total of 2565 records were identified and screened from the search for systematic reviews and guidelines for the general population and the Web-based search. Fourteen records met the eligibility criteria: two guidelines from the United States on screening for chronic hepatitis $\mathrm{B}$, three guidelines addressing treatment of chronic hepatitis B virus infection, one systematic review and one guideline on screening for hepatocellular carcinoma, one systematic review and one guideline on preventing hepatitis B virus infection among neonates, one systematic review on the adverse effects of hepatitis B vaccination, one systematic review on improving immunization rates and three guidelines on vaccinating against hepatitis B. In the search for articles relevant to immigrants and hepatitis B, we identified 148 articles addressing the following areas: epidemiology, screening, knowledge and compliance, treatment, and vaccination in the immigrant population.

\section{How does hepatitis B virus affect immigrant populations?}

Canada has low rates of hepatitis $B$ virus infection and an overall seroprevalence of chronic infection less than $0.5 \%$. Over the past 40 years, most immigrants who have arrived in Canada (>70\% of 250000 per year) have originated from countries with intermediate or high rates of endemic hepatitis B (Table 6A). ${ }^{109,160}$ These immigrants have an overall seroprevalence of chronic infection with hepatitis B virus of about $4 \% .^{40,158}$ People with chronic hepatitis B virus infection have a $15 \%-25 \%$ lifetime risk of dying from cirrhosis and hepatocel- lular carcinoma. ${ }^{157,161}$ They are typically asymptomatic until they present with end-stage liver disease or hepatocellular carcinoma several decades after infection. Hepatocellular carcinoma is one of the most fatal types of cancer, and the five-year survival rate is less than $11 \%$, because symptoms usually appear only at an advanced stage, when the disease is nontreatable. ${ }^{162}$ The growing pool of asymptomatic, undetected and untreated hepatitis $B$ virus infection in the immigrant population (8000 to 26000 new imported infections per year) is likely why mortality is higher among immigrants than in the Canadian-born population: 1.8-3.8 times higher from viral hepatitis and 2.2-4.9 times higher from hepatocellular carcinoma. ${ }^{10}$ The burden of undetected chronic infection with hepatitis B virus in immigrants is likely also in part responsible for the 8.4-fold higher mortality from chronic infection with hepatitis B virus and the 2.2-fold higher incidence of hepatocellular carcinoma over the past 30 years in Canada (between 1969 and 1997). ${ }^{163,164}$

Young children living in a household that includes someone with chronic hepatitis $\mathrm{B}$ virus infection have rates of acquiring new infection of $1 \%-2 \%$ per year during the first decade of life. ${ }^{109}$ Immigrant children, therefore, are at risk of acquiring hepatitis B in Canada, as they are more likely to live in a household that includes someone with silent hepatitis B virus infection. Many immigrant children have not been vaccinated in the universal childhood immunization program because they are too young or arrived after the age of vaccination. This risk was highlighted in a study in which Quebec's school-based vaccination program (for children in grade 4) was evaluated 10 years after initiation. In that study, rates of acute infection with hepatitis B virus decreased in all age groups except those 10 years of age and younger, and $53 \%$ of these cases occurred in foreign-born children. ${ }^{165}$ The study underscored both the need to provide protection against

Table 6A: Global patterns of chronic infection with hepatitis B virus (HBV) and hepatocellular carcinoma

\begin{tabular}{|c|c|c|c|}
\hline Disease characteristic & High prevalence & Intermediate prevalence & Low prevalence \\
\hline Geographic distribution & $\begin{array}{l}\text { Southeast Asia, China, } \\
\text { Pacific Islands, sub- } \\
\text { Saharan Africa, Alaska, } \\
\text { Peru, northwest Brazil }\end{array}$ & $\begin{array}{l}\text { Mediterranean basin, } \\
\text { eastern Europe, central } \\
\text { Asia, Japan, Amazon } \\
\text { basin, Middle East }\end{array}$ & $\begin{array}{l}\text { United States and } \\
\text { Canada, western and } \\
\text { northern Europe, } \\
\text { Australia, New Zealand }\end{array}$ \\
\hline Percentage of global population, $\%$ & 45 & 43 & 12 \\
\hline Predominant age at acquisition of infection & $\begin{array}{l}\text { Perinatal (vertical) and } \\
\text { early childhood } \\
\text { (horizontal) }\end{array}$ & All age groups & Adult \\
\hline $\begin{array}{l}\text { Likelihood of chronic infection after acute } \\
\text { infection with } \mathrm{HBV}, \% \text { * }\end{array}$ & $80-90$ & $30-60$ & $<5$ \\
\hline $\begin{array}{l}\text { Lifetime probability of infection } \\
\text { (immunity), \% }\end{array}$ & $>60$ & $20-60$ & $<20$ \\
\hline
\end{tabular}

* Likelihood that chronic infection with HBV will develop after acute infection with HBV is inversely proportional to age at acquisition. The earlier the infection is acquired, the earlier hepatocellular carcinoma will develop. ${ }^{109,160}$ 
hepatitis B as early as possible for children (possibly changing to routine infant rather than childhood vaccination programs) and the need to provide catch-up vaccination for immigrants.

\section{Does screening for chronic infection with hepatitis B virus decrease morbidity and mortality?}

\section{Screening tests}

Serologic tests to detect hepatitis B virus are inexpensive, and commercially available tests are sensitive and specific. ${ }^{166}$ Antigens and antibodies associated with hepatitis B virus infection include hepatitis B surface antigen and its corresponding antibody, antibody to hepatitis B core antigen, and hepatitis B e antigen and its corresponding antibody. The most frequently used serologic markers to differentiate acute, resolving and chronic infection are heptatitis B surface antigen, the antibody to hepatitis B core antigen (immunoglobulin $\mathrm{M}$ for acute, immunoglobulin $\mathrm{G}$ for chronic or resolved) and the antibody to hepatitis B surface antigen; they should be used for initial screening for hepatitis B virus infection. ${ }^{167}$ The antibody to hepatitis B core antigen is a useful marker in the diagnosis of hepatitis B virus infection, as it appears during the course of acute infection and usually persists for life in the presence of either chronic infection (in which case the person is also positive for heptatitis B surface antigen) or resolved infection, with or without the presence of concomitant antibody to hepatitis B surface antigen.

\section{Relative benefits and harms of treatment}

Several antiviral agents that suppress chronic hepatitis B virus infection have become available over the past 10 years. Although follow-up with the newer antiviral agents has not been long enough to show improved clinical outcomes, these agents have uniformly been shown to decrease surrogate markers of chronic liver disease (normalization of alanine aminotransferase, decrease in hepatitis B DNA, loss of hepatitis B e antigen and even loss of hepatitis B surface antigen). ${ }^{168-170}$ However, in a landmark randomized controlled trial of lamividuine versus placebo in patients with advanced liver disease and high-level viral replication, the risk of progressive liver failure (hazard ratio $0.45, p=0.02$ ) and of hepatocellular carcinoma (hazard ratio $0.49, p=$ 0.047 ) were both lower in the lamivudine arm than the placebo arm after a mean of 32 months of treatment (Table 6B). ${ }^{168}$ Lamivudine is well tolerated, but with prolonged use as a single agent, resistance develops progressively. Since publication of that trial, several other antiviral agents (adefovir, entecavir, telbivudine and tenofovir) have become available, all of which are relatively well tolerated and more potent than lamivudine. ${ }^{169,171-173}$ Treatment of chronic hepatitis B virus infection is rapidly evolving and complex; the decision of who should be treated, when to initiate therapy and which medication to use should be made by professionals with expertise in this area. ${ }^{171}$

\section{Effectiveness of surveillance for hepatocellular carcinoma}

Certain people with chronic hepatitis B virus infection (i.e.,

Table 6B: Summary of findings for efficacy of lamivudine to decrease mortality in patients with chronic HBV infection

Patient or population: "Adults > $16 \mathrm{yr}$, positive for HBsAg for at least 6 mo, and either positive or negative for HBeAg, HBV DNA $\geq 10^{5} \mathrm{IU} / \mathrm{mL}$ at screening, who had a liver biopsy showing an Ishak fibrosis score of at least 4 (where 0 indicates no fibrosis and 6 indicates cirrhosis) at screening or during the previous 2 yr." ${ }^{168}$

Setting: Multicentre trial with patients from Taiwan, Hong Kong, China, Singapore, Thailand and Australia Intervention: Lamivudine treatment

Comparison: Placebo

Source: Liaw YF, Sung JJ, Chow WC, et al. Lamivudine for patients with chronic hepatitis B and advanced liver disease. N Engl J Med 2004;351:1521-31. ${ }^{168}$

\begin{tabular}{|c|c|c|c|c|c|c|}
\hline \multirow[b]{2}{*}{ Outcome (period of follow-up) } & \multicolumn{2}{|c|}{ Absolute effect } & \multirow[b]{2}{*}{$\begin{array}{l}\text { Relative effect } \\
\quad(95 \% \mathrm{Cl})\end{array}$} & \multirow[b]{2}{*}{$\begin{array}{l}\text { No. of } \\
\text { participants } \\
\text { (studies) }\end{array}$} & \multirow[b]{2}{*}{$\begin{array}{l}\text { GRADE quality } \\
\text { of evidence }\end{array}$} & \multirow[b]{2}{*}{$\begin{array}{l}\text { Comments } \\
(95 \% \mathrm{Cl})\end{array}$} \\
\hline & $\begin{array}{l}\text { Risk for } \\
\text { control group }\end{array}$ & $\begin{array}{l}\text { Difference with } \\
\text { lamivudine } \\
(95 \% \mathrm{Cl})\end{array}$ & & & & \\
\hline $\begin{array}{l}\text { Increase in Child-Pugh score } \\
\text { (median } 32.4 \mathrm{mo} \text { ) }\end{array}$ & 88 per 1000 & $\begin{array}{l}54 \text { fewer per } 1000 \\
(70 \text { fewer to } 23 \\
\text { fewer per } 1000 \text { ) }\end{array}$ & $\begin{array}{c}\text { RR } 0.39 \\
(0.20-0.74)\end{array}$ & $651(1)$ & Moderate* & $\begin{array}{l}\text { NNT } 19 \\
(15-44)\end{array}$ \\
\hline $\begin{array}{l}\text { Hepatocellular carcinoma } \\
\text { incidence (median } 32.4 \text { mo) }\end{array}$ & 74 per 1000 & $\begin{array}{l}35 \text { fewer per } 1000 \\
(54 \text { fewer to } \\
1 \text { more per } 1000)\end{array}$ & $\begin{array}{c}\text { RR } 0.52 \\
(0.27-1.01)\end{array}$ & $651(1)$ & Moderate* & $\begin{array}{l}\text { NNT not } \\
\text { statistically } \\
\text { significant }\end{array}$ \\
\hline $\begin{array}{l}\text { Serious adverse eventst } \\
(5 \mathrm{yr})\end{array}$ & 177 per 1000 & $\begin{array}{l}53 \text { fewer per } 1000 \\
(92 \text { fewer to } \\
5 \text { more per } 1000)\end{array}$ & $\begin{array}{c}\text { RR } 0.70 \\
(0.48-1.03)\end{array}$ & $651(1)$ & Moderate* & $\begin{array}{l}\text { NNT not } \\
\text { statistically } \\
\text { significant }\end{array}$ \\
\hline
\end{tabular}

Note: $\mathrm{Cl}$ = confidence interval; GRADE = Grading of Recommendations Assessment, Development and Evaluation; HBeAg = hepatitis B e antigen; HBsAg = hepatitis B surface antigen; HBV = hepatitis B virus; NNT = number needed to treat; RR = relative risk.

*Data were analyzed by GlaxoSmithKline; article written by committee including employees.

†Adverse events were considered to be serious if the investigator determined that they jeopardized the patient's health, were life-threatening or would result in admission to hospital, disability or death. 
those with cirrhosis, Asian men $>40$ years of age, Asian women $>50$ years of age, Africans $>20$ years of age and people with a family history of hepatocellular carcinoma) should be screened for hepatocellular carcinoma with ultrasonography and $\alpha$-fetoprotein serologic testing every six months. ${ }^{172,174}$ This screening will detect cancer at an earlier stage, when it may be amenable to therapeutic intervention resulting in improved survival (five-year disease-free survival of about $50 \%$ v. $0 \%-10 \%)$. $^{162,173}$ In a randomized controlled trial of screening every six months with ultrasonography and $\alpha$-fetoprotein testing versus no screening in more than 18000 Chinese people with positive results for hepatitis B markers, those in the screening arm had a 37\% reduction in hepatocellular carcinoma-related mortality after a mean follow-up of five years. This was despite suboptimal adherence rates $(<60 \%)$ by the end of the study and represents the minimum benefit that can be expected from surveillance. ${ }^{175}$

\section{Effectiveness of screening programs for chronic infection with hepatitis B virus (pregnant women)}

Screening for chronic infection with hepatitis B is routinely recommended for pregnant women in Canada and the United States. ${ }^{11,176,177}$ There is good evidence that screening and giving immunoprophylaxis to infants born to mothers with chronic hepatitis B virus infection markedly decreases transmission to the newborn. In a recent meta-analysis, the risk of transmission of hepatitis B virus was $92 \%$ lower $(95 \%$ confidence interval [CI] 83\%-97\%) among infants born to mothers with chronic hepatitis B virus infection who received hepatitis B vaccine and hepatitis $B$ immunoglobulin within 12 hours of birth relative to those whose mothers received placebo. ${ }^{178}$

\section{Does vaccination decrease morbidity and mortality from hepatitis B?}

Hepatitis B vaccine (which is about $88 \%$ effective in preventing transmission) has been available in Canada since 1982, and a universal childhood hepatitis vaccination program has been recommended and operating in most provinces since 1991. ${ }^{11,177,179}$ It is an effective vaccine that substantially decreases the risk of acute or chronic hepatitis B virus infection and hepatocellular carcinoma. It is well tolerated, with only mild and transient adverse events. Additionally, several studies have demonstrated no link between hepatitis B vaccine and multiple sclerosis or other neurologic or rheumatologic disorders. ${ }^{109,111,177,179}$

\section{Effectiveness of hepatitis B vaccination to decrease morbidity and mortality}

\section{Children}

Several cohort studies in settings where the incidence of hepatitis B is high have shown the effectiveness of universal infant and childhood vaccination programs in decreasing the incidence of acute hepatitis B, of chronic infection with hepatitis B virus and of hepatocellular carcinoma. The incidence of acute hepatitis B virus infection decreased by $93.5 \%$, to $1 / 15$ th the initial rate (i.e., from 215 to 14 per 100000 population) over a four-year period in an Alaskan Native population after $90 \%$ vaccine coverage rate of the whole popuation. ${ }^{180}$ The relative risk of chronic infection with hepatitis B virus among vaccinated preschoolers relative to historical school-aged controls ranged from 0.1 to 0.34 in four South Pacific islands. ${ }^{181}$ Finally, after 15 years of follow-up, a universal infant and childhood vaccination program in Taiwan showed a dramatic decrease in seropositivity for hepatitis B surface antigen (from $9.8 \%$ to $0.7 \%$ ) and a $49 \%$ decrease in incidence of hepatocellular carcinoma, to just over half the initial rate (from 0.7 to 0.36 per 100000 population) (Table 6C)..$^{182-184}$

\section{Adults}

Hepatitis B vaccination in adults is also effective in decreasing acquisition of acute hepatitis B. A recent meta-analysis of the effectiveness of hepatitis B vaccination among health care workers (in countries where the incidence of hepatitis B virus infection is low) showed that vaccination decreased the acquisition of acute infection by $68 \%$ (95\% CI 35\%-84\%) relative to those who were unvaccinated. ${ }^{185}$ Because in adults it is uncommon for chronic infection with hepatitis B virus to develop following acute infection $(<5 \%)$, we were unable to identify any studies that measured the effectiveness of vaccine to decrease morbidity or mortality associated with chronic infection with hepatitis B virus in adults.

\section{Prevaccination screening for prior immunity}

From $50 \%$ to $80 \%$ of adults from countries where hepatitis B is highly endemic (prevalence of hepatitis B surface antigen $\geq$ $8 \%$ ) and $20 \%$ to $30 \%$ of adults from countries where hepatitis B is moderately endemic (prevalence of hepatitis B surface antigen 2\%-7\%) will have serologic evidence of prior infection with and thus immunity to hepatitis B virus (Table 6B). Cost-effectiveness studies have shown that the "breakpoint seroprevalence" of prior hepatitis B infection, above which prevaccination screening for prior immunity is worth doing, ranges from $17 \%$ to $35 \% .^{186,187}$ Therefore, it would likely be cost-effective to do prevaccination screening for prior infection among adult immigrants originating from countries that are moderately to highly endemic for hepatitis B. ${ }^{188}$ In two studies of refugee children with mean ages of 7 and 10 years, respectively, from several different world regions (Africa, former Soviet Union and Yugoslavia, Asia), the seroprevalence of hepatitis B surface antigen was $6.5 \%$ and $4 \%$, respectively, and the prevalence of prior infection was $30 \%$ and $21 \%$, respectively. ${ }^{189,190}$ Although these data might not be representative of all immigrant children, they suggest that it would also be cost-effective to do serologic testing for prior infection before vaccinating children.

\section{Clinical considerations}

\section{What are the potential implementation issues?}

Studies in immigrant populations have shown that they have relatively little knowledge $(40 \%-60 \%)$ of the importance of hepatitis B virus infection and its long-term consequences. 
Table 6C: Summary of findings for efficacy of universal childhood vaccination to decrease chronic HBV infection

Patient or population: Taiwanese children $<15$ years old, born after HBV vaccination program was implemented, and adolescents and young adults $>15-20$ years old, born before HBV vaccination program began

Setting: Chronic infection with HBV measured before and after implementation of vaccination program in Taiwan

Intervention: Perinatal and childhood HBV vaccination*

Comparison: No HBV vaccination

Source: $\mathrm{Ni}$ YH, Chang MH, Huang LM, et al. Hepatitis B virus infection in children and adolescents in a hyperendemic area: 15 years after mass hepatitis B vaccination. Ann Intern Med 2001;135:796-800. ${ }^{182}$

\begin{tabular}{|c|c|c|c|c|c|c|}
\hline Outcome & \multicolumn{2}{|c|}{ Absolute effect } & $\begin{array}{l}\text { Relative effect } \\
\qquad(95 \% \mathrm{Cl})\end{array}$ & $\begin{array}{l}\text { No. of } \\
\text { participants } \\
\text { (studies) }\end{array}$ & $\begin{array}{l}\text { GRADE quality } \\
\text { of evidence }\end{array}$ & $\begin{array}{l}\text { Comments } \\
(95 \% \mathrm{Cl})\end{array}$ \\
\hline $\begin{array}{l}\text { HBsAg carriage, } \\
<15 \text {-year-olds }\end{array}$ & 98 per 1000 & $\begin{array}{c}91 \text { fewer per } 1000 \\
\text { (94 to } 85 \text { fewer per } 1000 \text { ) }\end{array}$ & $\begin{array}{c}\text { RR } 0.07 \\
(0.04-0.13) \dagger\end{array}$ & $\begin{array}{l}2557 \\
(1)\end{array}$ & High $\ddagger$ & $\begin{array}{l}\text { NNT } 11 \\
(11-12)\end{array}$ \\
\hline
\end{tabular}

Note: anti-HBC = hepatitis B core antibody; $\mathrm{Cl}=$ confidence interval; GRADE = Grading of Recommendations Assessment, Development and Evaluation; HBsAg = hepatitis B surface antigen; HBV = hepatitis B virus; NNT = number needed to treat; RR = relative risk.

* "Taiwan's mass-vaccination program against HBV was launched in July $1984 .{ }^{24,183}$ For first 2 years, program covered only neonates born to mothers who were HBsAg carriers, but it was extended to all neonates in July 1986, to preschool children in July 1987, to primary-school children in 1988 , to middle-school children in 1989 , and to adults in 1990."

†Because of uncertainty regarding the sampling method, it is unclear to which populations these results can be generalized

‡Upgraded two levels for large effect sizes.

Low proportions $(<50 \%)$ of at-risk immigrants have been screened for hepatitis B. ${ }^{191,192}$ Similarly, low proportions of immigrants are vaccinated (24\%-76\%) against hepatitis B, and vaccination rates are lower among adults than among children. ${ }^{191-193}$ Vaccination rates can be enhanced by reducing language and cultural barriers, educating immigrant and refugee populations and improving social supports for using reminder and recall interventions (these being most effective but also most costly). ${ }^{155,193,194}$ These barriers and limitations need to be considered to improve uptake of screening and vaccination programs among immigrants. In general, when provided with the proper education and access, immigrants and refugees seem to accept vaccines with very little "antivaccination" sentiment. ${ }^{193-195}$ No data on how acceptance and compliance rates differ between cultures are available for hepatitis B treatment. Cultural and language barriers might need to be overcome to optimize the clinical impact of antiviral therapy and to minimize drug-resistant hepatitis B mutants.

\section{Recommendations of other groups}

With regard to screening, all pregnant women are screened for chronic hepatitis B virus infection in Canada and the United States to prevent transmission to their neonates. ${ }^{17,179}$ The US Centers for Disease Control and Prevention have also recently recommended screening all immigrants originating from countries where hepatitis B is endemic (prevalence of hepatitis B surface antigen $\geq 2 \%$ ). ${ }^{196}$

With regard to vaccination, hepatitis B vaccination is rou- tinely given to all children (age differs from province to province) as part of the national immunization program in Canada. There is no routine catch-up vaccine for immigrants; however, it is recommended that children younger than seven years whose families have immigrated to Canada from areas where prevalence of hepatitis B is high be targeted for vaccination. ${ }^{159,111}$

Our recommendations highlight the need to screen for chronic hepatitis B virus infection and to vaccinate susceptible high-risk immigrant groups.

\section{Take-home messages}

- Among newly arrived immigrants and refugees, the prevalence of chronic hepatitis B virus infection is about 3\%, as compared with $0.5 \%$ in the Canadian-born population.

- The mortality rate from chronic viral hepatitis and hepatocellular carcinoma is higher among immigrants than in the Canadian-born population, likely primarily because of greater prevalence of undetected and untreated chronic hepatitis B virus infection.

- Immigrants would benefit from screening for chronic hepatitis $B$ virus infection and vaccination against hepatitis B.

For the complete evidence review for hepatitis B in immigrant populations, see Appendix 5, available at www.cmaj.ca/lookup /suppl/doi:10.1503/cmaj.090313/-/DC1. 


\section{Tuberculosis}

Tuberculosis is an airborne transmissible disease that causes a substantial burden to patients, their contacts and society. Although tuberculosis is relatively uncommon in Canada (1621 cases, or about 5 per 100000 population, reported in 2006), it is costly (\$58 million in direct costs in Canada in 2004), treatment is lengthy, many patients require admission to hospital, and the mortality rate among patients with tuberculosis is still high $(11 \%) .{ }^{197,198}$

The foreign-born population bears a disproportionate burden of tuberculosis in Canada: $65 \%$ of all cases of active tuberculosis occur in foreign-born patients, although they make up only $20 \%$ of the population. ${ }^{41}$ This is because most recent immigrants and refugees originate from countries with a high incidence of tuberculosis, and up to half of them harbour latent tuberculosis infection and are at risk for development of active tuberculosis. ${ }^{198}$ Successful control of tuberculosis in Canada will depend on decreasing the rates of tuberculosis in the foreignborn population. We conducted a review to quantify the burden of tuberculosis in the migrant population, to identify those at highest risk for development of active tuberculosis, to describe the effectiveness of screening and treatment programs for latent tuberculosis, to identify barriers or challenges to implementation of such programs and to highlight possible interventions to improve these programs. The recommendations of the Canadian Collaboration for Immigrant and Refugee Health on preventing tuberculosis are outlined in Box 7A.

\section{Methods}

We used the 14-step approach developed by the Canadian Collaboration for Immigrant and Refugee Health ${ }^{16}$ (summarized in section 3 of this article, above). We considered the epidemiology of the disease in immigrant populations, as well as potential key clinical actions. We searched MEDLINE, Embase, CINAHL, the Cochrane Library and other sources from Jan. 1, 1996, to Jan. 1, 2010. Detailed methods, search terms, case studies and clinical considerations can be found in the complete evidence review for tuberculosis, as presented by Greenaway and associates. ${ }^{42}$

\section{Results}

In the search for systematic reviews and guidelines for immigrants, we found nine documents that met the eligibility criteria: two guidelines from the United States that addressed screening for latent tuberculosis infection in immigrants, three articles that addressed tuberculosis screening issues in foreign-born patients, one review of postlanding surveillance in Canada and three narrative reviews that recommended screening for latent tuberculosis infection in immigrants and refugees. However, none had used a systematic review method.

In the search for systematic reviews and guidelines for research involving tuberculosis in the general population, we

\section{Box 7A: Recommendations from the Canadian Collaboration for Immigrant and Refugee Health: tuberculosis}

Children: Screen children and adolescents $<20$ years of age from countries with a high incidence of tuberculosis (smearpositive pulmonary tuberculosis $>15$ per 100000 population) as soon as possible after their arrival in Canada with a tuberculin skin test, and recommend treatment for latent tuberculosis infection if results are positive, after ruling out active tuberculosis.

Adults: Screen all refugees between 20 and 50 years of age from countries with a high incidence of tuberculosis as soon as possible after their arrival in Canada with a tuberculin skin test. Screen all other adult immigrants who have risk factors that increase the risk of active tuberculosis by means of a tuberculin skin test, and recommend treatment for latent tuberculosis infection if results are positive, after ruling out active tuberculosis.

\section{Basis of recommendations}

\section{Balance of benefits and harms*}

The decision about whom to screen and offer treatment for latent tuberculosis is based on the balance between the potential benefit of treatment (decreasing the lifetime risk of active tuberculosis, which is influenced by age, presence of underlying medical conditions and immigration category) versus the potential harm of hepatotoxicity (which increases with age) and the poor effectiveness of isoniazid in many settings because of suboptimal uptake of screening and treatment. For several groups, screening for latent tuberculosis should be routinely performed, and those with positive results should be offered treatment. These groups are children from countries with a high incidence of tuberculosis (number needed to treat [NNT] 20-26, number needed to harm [NNH] 134-268), adults with risk factors for active tuberculosis (NNT 3-20, NNH variable) and refugees $<50$ years of age (NNT 15-26, NNH 49). Screening for latent tuberculosis and offering treatment could also be considered for adult refugees 50-65 years of age (NNT 20-51, NNH 9-18) and other adults without underlying medical conditions $<65$ years of age if adherence to treatment can be assured and hepatotoxicity carefully monitored to minimize harms. A decision to screen is a decision to offer treatment and to ensure adherence to treatment with appropriate counselling and monitoring.

\section{Quality of evidence}

High

\section{Values and preferences}

The committee attributed more value to screening and treating latent tuberculosis infection to prevent active disease in patients and to prevent transmission of active disease and less value to the practitioner burden of screening and counselling.

*Estimated number needed to treat (NNT) and number needed to harm (NNH) are based on the following assumptions: seven years after arrival, the annual risk of active tuberculosis is $0.1 \%$; the relative risk of active tuberculosis is highest upon arrival and decreases with time (relative risk 5.1, compared with 1.4 seven years after arrival); the patient will live to age 80 years; the efficacy of isoniazid is $90 \%$; and adherence is $70 \%$. 
identified 3968 articles, of which 18 met the eligibility criteria. These reviews addressed diagnostic tests for latent tuberculosis infection, the effect of bacille Calmette-Guérin on the tuberculin skin test, screening, factors increasing the risk of active tuberculosis, efficacy of treatment for latent tuberculosis infection and adherence to treatment. They included four US guidelines on controlling tuberculosis and treating latent tuberculosis infection. We identified the Canadian tuberculosis standards ${ }^{198}$ through the Web-based search. In the search for articles pertinent to tuberculosis in immigrants, we identified 3073 primary articles, of which 609 were relevant, addressing the following areas: epidemiology, diagnosis, screening, adherence and treatment for latent tuberculosis infection in immigrants.

\section{What is the burden of tuberculosis in immigrant populations?}

More than one billion people are infected with latent tuberculosis, which results in 9.2 million new active cases and 1.5 million deaths per year (> 95\% of these occurring in low- to middle-income countries). ${ }^{199}$ Canada is a low-incidence country, with an overall rate of active tuberculosis of five cases per 100000 population. ${ }^{41,200,201}$ Most of these cases (> 65\%) occur in foreign-born patients, among whom the incidence of tuberculosis is 20 times that in the non-Aboriginal Canadian-born population (16 v. 0.8 cases per 100000 population), but with rates as high as 500 times greater in certain subgroups of immigrants. ${ }^{202-204}$ In the past 40 years, most new immigrants have originated from high-incidence countries (i.e., $>15$ cases of smear-positive pulmonary tuberculosis per 100000 population), $30 \%-50 \%$ of whom are infected with latent tuberculosis, which has resulted in a reservoir of about 1.5 million people in Canada with latent tuberculosis infection who are at risk for development of active tuberculosis. ${ }^{198}$

Among people with positive results for tuberculin skin tests who live in a low-incidence country and have no risk factors, the estimated annual probability of development of active tuberculosis is only $0.1 \%$ per year. This means that active disease will develop in only $5 \%-10 \%$ of those with latent tuberculosis infection. ${ }^{205}$ Recent transmission of tuberculosis confers an increased risk of active tuberculosis. The highest risk of active tuberculosis occurs in the first year after exposure and decreases to the baseline risk $\left(0.1 \%\right.$ per year) 5 to 10 years after exposure. ${ }^{198,206}$

The strongest predictors for development of active tuberculosis in immigrant populations are global region of origin, immigration category (e.g., refugee), the presence of underlying medical comorbidity and the time since arrival.

\section{Region of origin}

Rates of tuberculosis are highest in immigrant populations that originate from world regions with the highest rates of tuberculosis, such as sub-Saharan Africa and Asia. Immigrants from these regions (which have rates of smear-positive pulmonary tuberculosis of 200 to 300 per 100000 population) are more likely to be heavily exposed to tuberculosis, to have positive results on tuberculin skin testing and to have been recently exposed to tuberculosis..$^{199,202-204}$

\section{Immigration category}

The risk of active tuberculosis in refugee populations is about double that in other immigrant populations. ${ }^{207-210}$ This difference is probably due to both a higher prevalence of latent tuberculosis infection and to having lived in crowded conditions that increase the likelihood of recent exposure to tuberculosis. ${ }^{211}$

\section{Presence of underlying comorbidity}

Underlying medical illnesses, especially any conditions that decrease local or systemic immunity, increase the rate of active tuberculosis to varying degrees (Table 7A), with HIV being the strongest risk factor. ${ }^{198}$ The issue of HIV screening for new immigrants and refugees is discussed in section 8 of this article, below.

\section{Time since arrival}

Rates of tuberculosis in immigrant and refugee populations, from all world regions, are highest within the first five years

Table 7A: Relative risk (RR) that active tuberculosis will develop in the presence of underlying medical conditions*

\begin{tabular}{|c|c|}
\hline Variable & $\mathrm{RR} \dagger$ \\
\hline \multicolumn{2}{|l|}{ High risk $(R R>6 \ddagger)$} \\
\hline AIDS & $110-170$ \\
\hline HIV infection & $10-110$ \\
\hline $\begin{array}{l}\text { Transplantation (related to } \\
\text { immunosuppressant therapy) }\end{array}$ & $20-74$ \\
\hline Leukemia, lymphoma & $1.0-35$ \\
\hline Silicosis & $1.5-33$ \\
\hline Chronic renal failure requiring hemodialysis & $1.6-25$ \\
\hline Carcinoma of head and neck & 16 \\
\hline Recent tuberculosis infection ( $\leq 2 \mathrm{yr}$ ) & 15 \\
\hline $\begin{array}{l}\text { Abnormal results of chest radiography: } \\
\text { fibronodular disease }\end{array}$ & $6-19$ \\
\hline Tumour necrosis factor $\alpha$ inhibitors & $1.7-9$ \\
\hline \multicolumn{2}{|l|}{ Intermediate risk (RR = 3-6‡) } \\
\hline Treatment with glucocorticoids & 4.9 \\
\hline Diabetes mellitus (all types) & $2.0-4.1$ \\
\hline Young when infected (0-4 yr) & $2.2-5$ \\
\hline \multicolumn{2}{|l|}{ Low-intermediate risk ( $R R=1.3-3 \ddagger)$} \\
\hline $\begin{array}{l}\text { Underweight ( }<90 \% \text { ideal body weight; } \\
\text { for most people, body mass index } \leq 20)\end{array}$ & $1.6-3$ \\
\hline Cigarette smoker (1 pack/d) & $2-3$ \\
\hline $\begin{array}{l}\text { Abnormal results of chest radiography: } \\
\text { granuloma }\end{array}$ & 2 \\
\hline Refugee & 2 \\
\hline \multicolumn{2}{|l|}{ Low risk $(R R=1) \S$} \\
\hline $\begin{array}{l}\text { Infected person, no known risk factor, } \\
\text { normal results of chest radiography } \\
\text { ("low-risk reactor") }\end{array}$ & 1 \\
\hline
\end{tabular}

*Adapted, with permission, from Greenaway and associates. ${ }^{42}$ tSee Greenaway and associates ${ }^{42}$ for sources for these RR values. $\ddagger$ Mean RR for each variable falls in this range. §Incidence of development of active tuberculosis $0.1 \% / \mathrm{yr}$. 
after arrival in a low-incidence region but decrease dramatically after the first year after arrival. Rates of active tuberculosis in the immigrant population, relative to five years after arrival, are 5 to 10 times greater in the first year and twofold greater one to four years after arrival. ${ }^{202-204}$ These higher rates are most likely caused by the effect of recent exposure to tuberculosis before arrival. A practical Web-based tool can be used to help calculate the lifetime risk of active tuberculosis on the basis of these factors (www.tstin3d.com/index.html). ${ }^{212}$

\section{Do screening and treatment for latent tuberculosis decrease morbidity from active tuberculosis?}

\section{Screening tests}

The tuberculin skin test and interferon gamma release assays are available for diagnosing latent tuberculosis infection. The sensitivity of these tests is estimated to be $70 \%-90 \%$, and the specificity for all tests is above $95 \%$, except for the tuberculin skin test in patients vaccinated with bacille Calmette-Guérin (specificity $60 \%$, because of cross-reactivity). ${ }^{213}$ With tuberculin skin tests, the likelihood of a false-positive result caused by bacille Calmette-Guérin decreases with time since vaccination, but it also depends on the age when the person was vaccinated. In the first 10 years after vaccination, up to $42 \%$ of patients vaccinated after two years of age will have positive results on tuberculin skin testing (the rate being lower among those vaccinated as neonates), but data on the rate of decline are conflicting. ${ }^{214-218}$ Among those receiving a tuberculin skin test more than 10 years after vaccination as a neonate, only $1 \%-2 \%$ of results will be positive, compared with $21 \%$ for those vaccinated after two years of age. Interpreting the results of a tuberculin skin test is therefore particularly difficult for

Table 7B: Summary of findings for isoniazid to prevent active tuberculosis

Patient or population: Varied: populations at risk for development of active tuberculosis, excluding HIV-positive patients; ${ }^{221}$ HIVpositive patients ${ }^{222}$

Setting: Varied: US psychiatric institutions, veterans' hospitals in United States, eastern Europe, Alaska, Hong Kong, India and elsewhere; ${ }^{221}$ Mexico, Haiti, United States, Zambia, Uganda and Kenya ${ }^{222}$

Intervention: Isoniazid treatment to prevent active tuberculosis

Comparison: No treatment

Sources: Smieja MJ, Marchetti CA, Cook DJ, et al. Isoniazid for preventing tuberculosis in non-HIV infected persons. Cochrane Database Syst Rev 1999;(1):CD001363. ${ }^{221}$ Bucher HC, Griffith LE, Guyatt GH, et al. Isoniazid prophylaxis for tuberculosis in HIV infection: a meta-analysis of randomized controlled trials. AIDS 1999;13:501-7.222

\begin{tabular}{|c|c|c|c|c|c|c|}
\hline \multirow[b]{2}{*}{ Outcome; risk category } & \multicolumn{2}{|c|}{ Absolute effect } & \multirow[b]{2}{*}{$\begin{array}{l}\text { Relative effect } \\
\qquad(95 \% \mathrm{Cl})\end{array}$} & \multirow[b]{2}{*}{$\begin{array}{l}\text { No. of } \\
\text { participants } \\
\text { (studies) }\end{array}$} & \multirow[b]{2}{*}{$\begin{array}{l}\text { GRADE quality } \\
\text { of evidence }\end{array}$} & \multirow[b]{2}{*}{$\begin{array}{l}\text { Comments } \\
(95 \% \mathrm{Cl})\end{array}$} \\
\hline & $\begin{array}{c}\text { Risk for } \\
\text { control group }\end{array}$ & $\begin{array}{l}\text { Difference } \\
\text { with isoniazid } \\
(95 \% \mathrm{Cl})\end{array}$ & & & & \\
\hline \multicolumn{7}{|l|}{ Active tuberculosis } \\
\hline Intermediate risk* & 17 per 1000 & $\begin{array}{l}10 \text { fewer per } \\
1000 \\
(12 \text { fewer to } \\
8 \text { fewer per } \\
1000 \text { ) }\end{array}$ & $\begin{array}{c}\text { RR } 0.40 \\
(0.31-0.52)\end{array}$ & $\begin{array}{c}73375 \\
(11)\end{array}$ & Moderate $\ddagger \S$ & $\begin{array}{l}\text { NNT } 99 \\
(86-123)\end{array}$ \\
\hline $\begin{array}{l}\text { Highly compliant } \\
\text { (> } 80 \% \text { of doses } \\
\text { taken)* }\end{array}$ & 10 per 10000 & $\begin{array}{l}7 \text { fewer per } \\
10000 \\
(8 \text { fewer to } \\
5 \text { fewer per } \\
10000 \text { ) }\end{array}$ & $\begin{array}{c}\text { RR } 0.20 \\
(0.13-0.31)\end{array}$ & $\begin{array}{c}15696 \\
(1)\end{array}$ & High & $\begin{array}{l}\text { NNT } 85 \\
(78-98)\end{array}$ \\
\hline High riskt & 53 per 1000 & $\begin{array}{l}32 \text { fewer per } \\
1000 \\
(40 \text { fewer to } \\
19 \text { fewer per } \\
1000 \text { ) }\end{array}$ & $\begin{array}{c}\text { RR } 0.40 \\
(0.24-0.65)\end{array}$ & $\begin{array}{c}1875 \\
(5)\end{array}$ & Moderateף & $\begin{array}{l}\text { NNT } 32 \\
(25-54)\end{array}$ \\
\hline $\begin{array}{l}\text { Hepatitis } \\
\text { (follow-up } 5 \mathrm{yr} \text { )** }\end{array}$ & 1 per 1000 & $\begin{array}{l}5 \text { more per } \\
1000 \\
(2 \text { more to } \\
11 \text { more per } \\
1000)\end{array}$ & $\begin{array}{c}\text { RR } 5.54 \\
(2.56-12)\end{array}$ & $\begin{array}{l}20874 \\
\text { (1) }\end{array}$ & Moderate & $\begin{array}{l}\text { NNH } 220 \\
(91-642)\end{array}$ \\
\hline \multicolumn{7}{|c|}{$\begin{array}{l}\text { Note: } \mathrm{Cl}=\text { confidence interval; GRADE = Grading of Recommendations Assessment, Development and Evaluation; NNH = number needed to harm; NNT = number } \\
\text { needed to treat; RR = risk ratio. } \\
\text { *Numbers taken from Smieja et al. }{ }^{221} \\
\text { †In } 1999 \text { systematic review of isoniazid for tuberculosis in HIV-positive patients, this was the risk among HIV-positive, tuberculin skin test-positive patients. } \\
\text { ‡Test for heterogeneity, } p=0.02 \text {. } \\
\text { §Only one study examined } 6 \text { months v. } 12 \text { months of isoniazid therapy; risk of hepatitis and active tuberculosis not significantly different between groups. } \\
\text { ๆDowngraded for directness, as the data were from developing countries. } \\
\text { **As reported by the International Union Against Tuberculosis Committee on Prophylaxis. }{ }^{223}\end{array}$} \\
\hline
\end{tabular}


children under 10 years of age. All HIV-positive patients should be screened for latent tuberculosis infection with a tuberculin skin test. ${ }^{198}$

Because there is no gold standard test for the diagnosis of latent tuberculosis infection, assessing and comparing the performance of these tests is challenging, especially when there are discrepancies. The major advantage of the tuberculin skin test is that the risk of active disease for different sizes of induration is well described, whereas very few prospective data exist for interferon gamma release assays. The most recent Canadian guidelines recommend using the tuberculin skin test as the primary screening tool for both adults and children and using interferon gamma release assays sequentially, after tuberculin skin testing, in people with a high likelihood of a false-positive result on the tuberculin skin test (i.e., low risk of tuberculosis infection). ${ }^{219}$ This recommendation is supported by a recent cost-effectiveness analysis. ${ }^{220}$ The major limitation of these tests is their inability to distinguish the $10 \%$ of people with latent tuberculosis infection in whom active tuberculosis will develop from the $90 \%$ in whom the disease will not develop.

All patients for whom the results of a tuberculin skin test are positive should undergo chest radiography to rule out active tuberculosis; they should also be questioned for symptoms of active tuberculosis (chronic cough, weight loss, fever, night sweats). If there is any suspicion of active tuberculosis, three samples of sputum or specimens from other sites (e.g., lymph node, cerebrospinal fluid) should be gathered for smear and culture before treatment for latent tuberculosis infection is started.

\section{Relative benefits and harms of treatment}

The efficacy of isoniazid relative to that of placebo in decreasing the likelihood that active tuberculosis will develop in people with latent tuberculosis infection has been estab- lished in a Cochrane review of 11 randomized controlled trials (relative risk $0.40,95 \%$ confidence interval $0.31-0.52$ ) (Tables 7B and 7C). ${ }^{221-224}$ The overall efficacy of isoniazid is $62 \%$ after 12 months of treatment, but efficacy increases to $93 \%$ among those who adhere to treatment (i.e., take $>80 \%$ of their doses). ${ }^{223}$ Although the efficacy of treatment for 9 versus 12 months has not been directly compared, a recent reanalysis showed that the maximal benefit of isoniazid was achieved at 9 months (Tables 7D). ${ }^{225}$ A study on the effect of resistance to isoniazid on the efficacy of isoniazid chemoprophylaxis showed that, at a mean prevalence of 7\%-10\% isoniazid resistance (the level in the immigrant population), isoniazid was the drug of choice and that only at very high rates of isoniazid resistance (>15\%-20\%) were other regimens preferred. ${ }^{226,227}$

Hepatotoxicity is a limitation of isoniazid therapy. It most commonly manifests as a transient, asymptomatic increase in liver function $(10 \%-20 \%)$, rarely causes clinical hepatitis $(0.5 \%)$ (which resolves when treatment with isoniazid is stopped $\left.^{224,228,229}\right)$ and very rarely causes fulminant hepatitis and liver failure leading to death or liver transplantation $(<0.01 \%) .{ }^{230,231}$ Initial higher overall rates of hepatotoxicity (1\%) were reported among adults in the 1970s, but these data were likely confounded by unrecognized underlying cirrhosis. Hepatotoxicity among patients taking isoniazid is greater among those with pre-existing liver disease, alcoholism, concomitant use of hepatotoxic drugs and older age. Although clinical and fulminant hepatitis are rare, they can occur at any age. This possibility underscores the importance of monthly monitoring for all patients and of teaching them to recognize the symptoms of hepatitis (nausea, vomiting, abdominal pain, jaundice) and to stop medication as soon as worrisome symptoms occur. ${ }^{230,231}$ Adequate time must be taken, through interpreters if necessary, to ensure that all patients are appropri-

Table 7C: Summary of findings for use of isoniazid for all age groups with latent tuberculosis infection

Patient or population: Patients with latent tuberculosis

Setting: Tuberculosis clinics in Memphis, Tennessee

Intervention: Isoniazid treatment to prevent active tuberculosis

Comparison: Younger people compared with older people

Source: Fountain FF, Tolley E, Chrsman CR, et al. Isoniazid hepatotoxicity associated with treatment of latent tuberculosis infection: a 7-year evaluation from a public health tuberculosis clinic. Chest 2005;128:116-23. ${ }^{224}$

\begin{tabular}{|c|c|c|c|c|c|c|c|c|c|}
\hline \multirow[b]{3}{*}{ Outcome } & \multicolumn{3}{|c|}{ Absolute effect } & \multirow{2}{*}{\multicolumn{2}{|c|}{$\begin{array}{l}\text { Relative effect } \\
\quad(95 \% \mathrm{Cl})\end{array}$}} & \multirow{3}{*}{$\begin{array}{l}\text { No. of } \\
\text { participants } \\
\text { (studies) }\end{array}$} & \multirow{3}{*}{$\begin{array}{l}\text { GRADE } \\
\text { quality of } \\
\text { evidence }\end{array}$} & \multirow{2}{*}{\multicolumn{2}{|c|}{$\begin{array}{c}\text { Comments } \\
(95 \% \mathrm{Cl})\end{array}$}} \\
\hline & \multirow{2}{*}{$\begin{array}{c}\begin{array}{c}\text { Risk for } \\
\text { control } \\
\text { group }\end{array} \\
\text { Age 25-34 }\end{array}$} & \multicolumn{2}{|c|}{$\begin{array}{l}\text { Difference with older age } \\
\qquad(95 \% \mathrm{Cl})\end{array}$} & & & & & & \\
\hline & & Age $35-49$ & Age $\geq 50$ & $\begin{array}{c}\text { Age } \\
35-49\end{array}$ & $\begin{array}{l}\text { Age } \\
\geq 50\end{array}$ & & & $\begin{array}{c}\text { Age } \\
35-49\end{array}$ & $\begin{array}{l}\text { Age } \\
\geq 50\end{array}$ \\
\hline
\end{tabular}

Note: $\mathrm{Cl}=$ confidence interval; GRADE = Grading of Recommendations Assessment, Development and Evaluation; NNH = number needed to harm; RR = risk ratio

* Fewer than 300 events. 
ately informed and understand the risks and benefits of isoniazid, and they must be given a clear description of what to do if symptoms arise.

\section{Clinical considerations}

\section{What should be considered in screening and treatment?}

\section{Children}

Children less than 11 years of age do not undergo prearrival radiographic screening. For this and several other reasons, they could benefit greatly from screening for latent tuberculosis infection. Children face many years of life in which active tuberculosis could develop, and they have a relatively low potential for hepatotoxicity. ${ }^{230,231}$ When active tuberculosis develops in children, it is often difficult to diagnose because it is more often paucibacillary or extrapulmonary, and young children (especially those younger than five years of age) are more likely susceptible to severe or rapidly progressive disease. ${ }^{232}$

\section{Refugees}

Refugee populations have consistently had about a two-fold greater risk of active tuberculosis than the immigrant population, at least within the first year after arrival. ${ }^{207-210} \mathrm{~A}$ higher prevalence of latent tuberculosis infection in the refugee population and having lived in crowded conditions, which increase the likelihood of recent exposure to tuberculosis, are contributing factors.

\section{What are the potential implementation issues?}

Barriers to the uptake of screening and completion of treatment for latent tuberculosis infection include a combination of patient, provider and institutional factors. Patient-related barriers include the stigma of tuberculosis and its association with HIV, linguistic barriers and difficulties getting to appointments because of inconvenient clinic locations or limited clinic hours. ${ }^{233-236}$ Provider-related barriers to offering screening to migrants are related to inadequate knowledge of which migrants should be screened and how they should be followed. ${ }^{237}$ Low adherence to treatment for latent tuberculosis infection is associated with barriers similar to those for screening for latent tuberculosis. These barriers include linguistic barriers, cultural taboos and stigmatization, low education level, perceived low risk of progression from latent tuberculosis infection to active disease, belief that positive results from tuberculin skin tests are due to bacille Calmette-Guérin, not wanting to undergo venipuncture and economic factors (costs of travel, lack of insurance, delays in obtaining insurance, missed days at work). ${ }^{234,235,238}$

Increased adherence to tuberculin skin test screening has been achieved with reminders to patients (e.g., letters, phone calls), education of patients and physicians, and novel strat-

Table 7D: Estimated numbers needed to treat for latent tuberculosis infection to prevent one case of active tuberculosis by age, time since arrival and adherence*

\begin{tabular}{|c|c|c|c|c|c|c|}
\hline \multirow{2}{*}{$\begin{array}{l}\text { Age, } \\
\text { yr }\end{array}$} & \multirow{2}{*}{$\begin{array}{c}\text { Time lived } \\
\text { in Canada, } \\
\text { yr }\end{array}$} & \multirow{2}{*}{$\begin{array}{l}\text { Cumulative lifetime } \\
\text { risk of active } \\
\text { tuberculosis, \% †‡ }\end{array}$} & \multicolumn{4}{|c|}{ Course of isoniazid completed§; NNT } \\
\hline & & & $100 \%$ & $70 \%$ & $50 \%$ & $30 \%$ \\
\hline \multirow[t]{3}{*}{10} & 0 & 8.1 & 14 & 20 & 28 & 46 \\
\hline & 2 & 7.5 & 15 & 22 & 30 & 50 \\
\hline & 5 & 7.1 & 16 & 23 & 31 & 52 \\
\hline \multirow[t]{3}{*}{20} & 0 & 7.1 & 16 & 23 & 32 & 52 \\
\hline & 2 & 6.5 & 18 & 25 & 35 & 57 \\
\hline & 5 & 6.1 & 18 & 26 & 36 & 60 \\
\hline \multirow[t]{3}{*}{35} & 0 & 5.6 & 20 & 29 & 40 & 66 \\
\hline & 2 & 5.0 & 23 & 32 & 45 & 75 \\
\hline & 5 & 4.6 & 24 & 34 & 48 & 80 \\
\hline \multirow[t]{3}{*}{50} & 0 & 4.1 & 28 & 39 & 55 & 91 \\
\hline & 2 & 3.5 & 32 & 46 & 64 & 107 \\
\hline & 5 & 3.1 & 36 & 51 & 71 & 118 \\
\hline \multirow[t]{3}{*}{65} & 0 & 2.6 & 44 & 62 & 87 & 144 \\
\hline & 2 & 2.0 & 57 & 81 & 113 & 188 \\
\hline & 5 & 1.6 & 68 & 97 & 136 & 226 \\
\hline
\end{tabular}

Note: NNT = number needed to treat.

*Adapted, with permission, from Greenaway and associates. ${ }^{42}$

†Assume $0.1 \%$ annual risk of infection, with a relative risk of development of active tuberculosis for each year since arrival of 5.08 for $<1$ year, 2.96 for 1.1-2 years, 2.35 for $2.1-3$ years, 2.06 for $3.1-4$ years, 1.87 for $4.1-5$ years, 1.89 for 5.1-6 years, and 1.36 for $6.1-7$ years (all $v .>7$ years) $203,205,224$

$\ddagger$ Assume individuals live to age 80 years.

§For $100 \%$ completion, assume $90 \%$ efficacy of isoniazid. ${ }^{221,223}$ 
egies, such as drive-by tuberculin skin test readings for taxi drivers. ${ }^{237,239}$ In one study, educating primary care providers about how and whom to screen for tuberculosis not only increased screening and identification of people with latent tuberculosis, but also increased identification of those with active tuberculosis. In this randomized clinical trial, screening rates were $0.4 \%$ in the nonintervention group and $57 \%$ in the intervention groups, and identification of both those with latent tuberculosis infection (9\% to 19\%) and those with active tuberculosis (34\% v. $47 \%$ ) was higher in the intervention group. ${ }^{237}$ Strategies that have increased adherence to treatment for latent tuberculosis infection in immigrant and refugee populations include patient reminders (calendar stickers for self-monitoring, phone calls, letters and directly observed therapy), adherence coaches who speak the same language as the patient, ongoing education of patients and providers, and cultural case management. ${ }^{43}$ Goldberg and colleagues ${ }^{240}$ found that when case managers were matched to the ethnic and linguistic background of patients and provided treatment for latent tuberculosis and monitoring during monthly home visits, adherence with treatment improved substantially over standard clinic-based management before the intervention ( $82 \%$ v. $37 \%$ ).

\section{Recommendations of other groups}

The Canadian Tuberculosis Committee, the Canadian Thoracic Society and the Canadian Paediatric Society recommend using a tuberculin skin test to screen the following groups from countries with a high incidence of tuberculosis: children younger than 15 years living in Canada for less than two years and people 15 years of age or older with factors increasing the risk of active tuberculosis or within two years after arrival if they have had known contact with tuberculosis (Table 7A). ${ }^{198}$ Our recommendations highlight the importance of screening for latent tuberculosis with Mantoux tests in high-risk immigrant groups.

\section{Take-home messages}

- Foreign-born people account for $65 \%$ of all those with active tuberculosis in Canada, and subgroups have up to a 500 -fold greater risk of active tuberculosis relative to the non-Aboriginal Canadian-born population.

- The Canadian Collaboration for Immigrant and Refugee Health recommends screening certain groups as soon as possible after arrival in Canada, with a tuberculin skin test, and initiating treatment for latent tuberculosis infection in those with a positive result, after ruling out active tuberculosis.

- Although isoniazid is highly efficacious in decreasing the development of active tuberculosis in those with latent tuberculosis infection, monitoring for hepatotoxicity is required for patients of all ages.

- Close monitoring is required for those over 50 years of age and those with pre-existing liver disease, alcoholism or concomitant use of hepatotoxic drugs.

- Adherence to screening and treatment for latent tuberculosis infection can be increased if delivered in a culturally sensitive manner.

For the complete evidence review for tuberculosis in immigrants, see www.cmaj.ca/lookup/doi/10.1503/cmaj.090302. 


\section{HIV}

An estimated 33.2 million women, men and children are infected with HIV worldwide. ${ }^{241}$ In Canada, populations at high risk for HIV include women and men from countries where HIV is endemic, ${ }^{242}$ who are at risk of heterosexual transmission and mother-to-child transmission. ${ }^{243}$ Although HIV testing is now part of the immigration medical examination, persons with HIV are not necessarily excluded from immigrating. Delayed disclosure of positive results by patients to their partners and practitioners, as well as the ongoing risk of HIV transmission attributable to a variety of factors (such as travel to the country of origin, where HIV could be endemic $^{244}$ ), remains a concern. We conducted an evidence review to guide primary care practitioners in the early detection, prevention and treatment of HIV for newly arriving immigrants. The recommendations of the Canadian Collaboration for Immigrant and Refugee Health on HIV screening and treatment are outlined in Box 8A.

\section{Methods}

We used the 14-step method developed by the Canadian Collaboration for Immigrant and Refugee Health ${ }^{16}$ (summarized in section 3 of this article, above). We considered the epidemiology of HIV in immigrant populations and defined clinical preventive actions (interventions), outcomes and key clinical questions. We searched MEDLINE, Embase, CINAHL, the Cochrane Library and other sources from Jan. 1, 1995, to Jan. 1, 2010. Detailed methods, search terms, case studies and clinical considerations can be found in the complete evidence review for HIV (Appendix 6, available at www.cmaj.ca/lookup/suppl/doi:10.1503/cmaj.090313/-/DC1).

\section{Box 8A: Recommendations from the Canadian Collaboration for Immigrant and Refugee Health: HIV}

Screen for HIV, with informed consent, all adolescents and adults from countries where HIV is prevalent (> $1 \%)$.

\section{Basis of recommendations}

\section{Balance of benefits and harms}

The decision to screen men and women for HIV is based on a dramatic reduction in mortality with treatment, e.g., with a combination of three versus two antiretrovirals (number needed to treat [NNT] 132, 95\% confidence interval [CI] 91-357) and reduction of high-risk behaviour (NNT 5, 95\% Cl 4-7). Prevalence of HIV infection is higher among immigrants from countries where HIV is prevalent $(>1 \%)$ than among other Canadians $(<0.18 \%)$. Harms included adverse drug reactions requiring change in regimen. Data on harms related to anxiety and possible discrimination related to HIV status are unavailable.

\section{Quality of evidence}

\section{Moderate}

\section{Values and preferences}

The committee attributed more value to identifying HIVpositive women and men for appropriate treatment, support and prevention and less value to uncertain risk of couple discord and risk of discrimination and less concern for burden of testing with informed consent.

\section{Results}

We initially identified eight papers, including a systematic review of HIV prevalence studies among sub-Saharan African refugees $^{245}$ and a systematic review of retention of African patients in antiretroviral treatment programs..$^{246}$ Although these papers detailed the complexities of HIV issues among immigrants, highlighting the need for sensitivity to sociocultural context in the interventions, none provided evidence of the benefits and harms of screening. Without restricting the search to papers specific to immigrants, we identified 13 systematic reviews and guideline articles providing evidence on screening for HIV. ${ }^{24-259}$ We identified and appraised longitudinal studies of antiretroviral treatment, ${ }^{260,261}$ a meta-analysis on behaviour change related to HIV screening tests, ${ }^{249}$ a meta-analysis on antiretroviral therapy $^{256}$ and a Cochrane systematic review on antiretroviral treatment. ${ }^{247}$ One hundred and four titles addressed the burden of HIV and barriers to care for immigrant populations.

\section{What is the burden of HIV in immigrant populations?}

The HIV infection rate is about 12.6 times higher among immigrants and refugees from countries where HIV is endemic than it is in the Canadian-born population, ${ }^{243}$ accounting for $7 \%$ of HIV cases in large urban centres. ${ }^{10}$ In subgroup mortality analyses of the Canadian Mortality Database, both male and female immigrants from the Caribbean had higher mortality from HIV infection (standardized mortality rates 4.2 for males and 27.4 for females). In a study based on screening of immigration applicants to Canada, $70 \%$ of those who tested positive were refugees or refugee claimants from regions where HIV is endemic (Table 8A), and the seropositivity among applicants from sub-Saharan Africa was above 3\%. ${ }^{262}$

Table 8A: Prevalence of HIV by region in 2007*

\begin{tabular}{ll}
\hline Region & $\begin{array}{c}\text { Prevalence of HIV, } \\
\%(95 \% \mathrm{Cl})\end{array}$ \\
\hline Sub-Saharan Africa & $5.0(4.6-5.5)$ \\
\hline Caribbean & $1.0(0.9-1.2)$ \\
\hline Eastern Europe and central Asia & $0.9(0.7-1.2)$ \\
\hline North America & $0.6(0.5-0.9)$ \\
\hline Latin America & $0.5(0.4-0.6)$ \\
\hline Oceania & $0.4(0.3-0.7)$ \\
\hline Middle East and north Africa & $0.3(0.2-0.4)$ \\
\hline Southeast Asia & $0.3(0.2-0.4)$ \\
Western and central Europe & $0.3(0.2-0.4)$ \\
\hline East Asia & $0.1(<0.2)$ \\
\hline
\end{tabular}

Note: $\mathrm{Cl}=$ confidence interval

*Adapted, with permission, from Joint United Nations Programme on HIVIAIDS (UNAIDS). ${ }^{241}$ 
Women 20-39 years of age accounted for $66 \%$ of positive HIV test reports among adult immigrant women in Canada in $2006 .{ }^{263}$ Most of these women had been exposed to HIV in areas where infection was endemic. Sexual transmission of HIV from a man to a woman is two to eight times more likely than transmission from a woman to a man. A woman's susceptibility to HIV infection is further increased if she or her partner has a sexually transmitted infection, if she has experienced genital trauma or if her partner is HIV-positive with a high viral load. ${ }^{264}$ Determinants of vulnerability to HIV include increased mobility (e.g., 1.5-1.8 times higher for mobile populations in South Africa) ${ }^{265}$ experience of warrelated violence (e.g., 10\%-12\% of women experiencing sexual violence in the Democratic Republic of Congo contract HIV) ${ }^{266}$ certain sexual practices (studies suggest a twofold risk of HIV with vaginal douching), ${ }^{267}$ limited knowledge about HIV and AIDS, and limited language proficiency. ${ }^{241}$

\section{Does screening for HIV decrease related morbidity and mortality?}

\section{Screening tests}

Most laboratories in Canada use a two-step testing strategy for HIV. Enzyme-linked immunosorbent assays are sensitive $(\geq 99 \%)$ and specific ( $\geq 99 \%$ ) for both HIV-1 and HIV-2 after about three weeks of infection. Risk-reduction counselling (e.g., regarding condom use) has been shown to be most effective when targeted to HIV-positive or high-risk women and men. ${ }^{249}$

\section{Relative benefits and harms of treatment}

In a meta-analysis of 27 studies on behaviour change in HIVpositive women and men, Weinhardt and associates ${ }^{249}$ found decreases in high-risk sexual behaviour (including unprotected intercourse) with provision of counselling and screening (Table $8 \mathrm{~B})$. We were unable to find quantitative data on anxiety or depression, changes in relationships with sexual partners or discrimination associated with false-positive results.

A meta-analysis by Enanoria and colleagues ${ }^{256}$ comparing three-drug and two-drug antiretroviral treatment showed a significant decrease in mortality (risk ratio $0.62,95 \%$ confidence interval 0.45-0.86) (Table 8C). In another meta-analysis, Siegfried and coauthors ${ }^{247}$ reported a relative risk of 1.3 for adverse events associated with antiretroviral treatment. However, most adverse events, including metabolic disturbances associated with cardiovascular events, can be ameliorated by changes in regimen or appropriate treatment. ${ }^{248}$ Evidence for a greater than $80 \%$ reduction in HIV transmission among HIV-discordant couples receiving antiretroviral treatment has been obtained from Spain, Thailand and Uganda. ${ }^{268}$ Combination antiretroviral therapy is associated with dramatic decreases in mortality, regardless of sex, race, age and risk factors for transmission. ${ }^{260}$ Recent studies have reported that voluntary counselling and testing for HIV are costeffective in populations with HIV prevalence above $0.1 \%{ }^{269}$

\section{Clinical considerations}

\section{What are the potential implementation issues?}

Refugees and refugee claimants may delay testing and/or

Table 8B: Summary of findings on how pretest counselling and screening affect high-risk behaviours

Patient or population: HIV-positive patients, mostly men

Setting: United States

Intervention: Counselling and screening tests

Comparison: No screening procedures

Source: Weinhardt LS, Carey MP, Johnson BT, et al. Effects of HIV counseling and testing on sexual risk behavior: a meta-analytic review of published research, 1985-1997. Am J Public Health 1999;89:1397-405. ${ }^{249}$

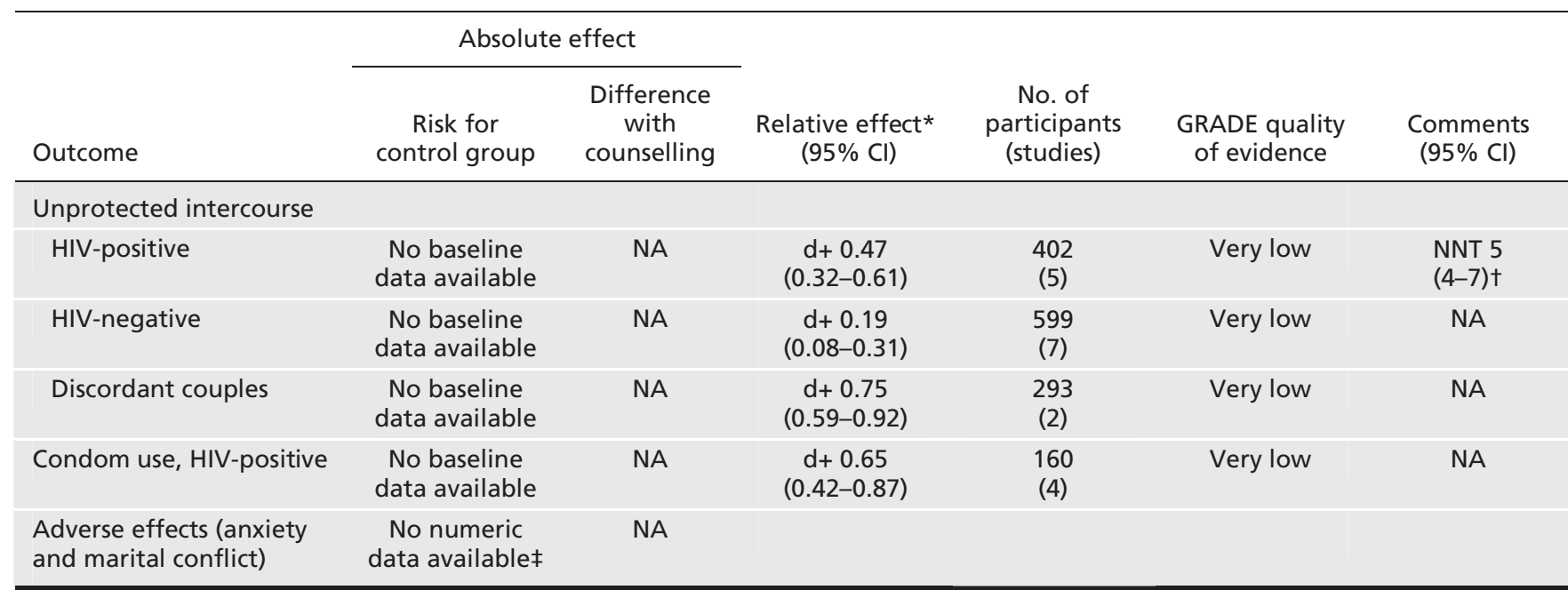

Note: $\mathrm{Cl}=$ confidence interval; $\mathrm{d}+=$ standardized mean difference index; GRADE = Grading of Recommendations Assessment, Development and Evaluation; NA = not applicable; NNT = number needed to treat.

*The value for $d+$, the standardized mean difference index, is computed by behaviour before and after HIV counselling and testing. An effect size of 0.20 is considered small and 0.5 is considered medium. Positive effect sizes indicate a reduction in high-risk sexual behaviour.

tWe are very uncertain about the estimate.

$\ddagger$ Qualitative studies have highlighted various issues but have also suggested that immigrants favour increased access to testing. 
Table 8C: Summary of findings for antiretroviral treatment of HIVIAIDS

Patient or population: Patients with HIV/ AIDS

Setting: Outpatient clinics in Australia, Europe and North America

Intervention: Treatment with three antiretrovirals

Comparison: Treatment with two antiretrovirals

Source: Enanoria WTA, Ng C, Saha SR, et al. Treatment outcomes after highly active antiretroviral therapy: a meta-analysis of randomised controlled trials. Lancet Infect Dis 2004;4:414-25. ${ }^{256}$

\begin{tabular}{|c|c|c|c|c|c|c|}
\hline Outcome & \multicolumn{2}{|c|}{ Absolute effect } & $\begin{array}{l}\text { Relative effect } \\
\quad(95 \% \mathrm{Cl})\end{array}$ & $\begin{array}{l}\text { No. of } \\
\text { participants } \\
\text { (studies) }\end{array}$ & $\begin{array}{l}\text { GRADE quality } \\
\text { of evidence }\end{array}$ & $\begin{array}{l}\text { Comments } \\
(95 \% \mathrm{Cl})\end{array}$ \\
\hline Death & 20 per 1000 & $\begin{array}{l}8 \text { fewer per } 1000 \\
(1 \text { to } 9 \text { fewer } \\
\text { per } 1000)\end{array}$ & $\begin{array}{c}\text { RR } 0.62 \\
(0.45-0.86)\end{array}$ & $\begin{array}{c}3979 \\
(15)\end{array}$ & Moderate* & $\begin{array}{l}\text { NNT } 132 \\
(91-357)\end{array}$ \\
\hline $\begin{array}{l}\text { Withdrawals due to } \\
\text { adverse effects }\end{array}$ & 100 per 1000 & $\begin{array}{l}81 \text { more per } 1000 \\
\text { (38 to } 198 \text { more } \\
\text { per } 1000 \text { ) }\end{array}$ & $\begin{array}{c}\text { RR 1.81 } \\
(1.19-2.79)\end{array}$ & $\begin{array}{l}6380 \\
(11)\end{array}$ & Low*‡ & $\begin{array}{l}\text { NNH } 12 \\
(6-53)\end{array}$ \\
\hline
\end{tabular}

Note: $\mathrm{Cl}=$ confidence interval; GRADE = Grading of Recommendations Assessment, Development and Evaluation; NNH = number needed to harm; NNT = number needed to treat; $R R=$ risk ratio.

*Consistency downgraded because test for heterogeneity was statistically significant.

tIndirect because viral load is an indirect outcome for mortality and morbidity.

$\ddagger$ Adverse events are an indirect outcome for severe side effects.

treatment because they fear failing to obtain legal immigration status if HIV-positive. ${ }^{45}$ Immigrants suffering from posttraumatic stress or depression could require additional social support and reassurance on many fronts before finding the treatment for HIV infection acceptable. Many HIV-positive immigrants adopt codes of silence, which precludes providing sex education to their children. ${ }^{45,270}$ This highlights a crucial role for primary care practitioners in providing education about sex and HIV to youth.

Limited education, lower health literacy, linguistic barriers, traditional and religious beliefs, ${ }^{45}$ psychological issues that stem from HIV-related stigma, practitioners' reluctance to offer screening tests and perceptions of low risk among women and men suffering from HIV have all been identified as factors impeding HIV testing. ${ }^{271} \mathrm{HIV}$-related stigma is magnified by structural inequities, sex roles, negative attitudes and discrimination by health care providers, and social attitudes in general. ${ }^{272}$ Strategies to improve access to care include sensitivity to emotional and trauma issues, provision of comprehensive and holistic care, presence of HIV-positive peer educators and integration of HIV information within settlement services. ${ }^{270}$ Qualitative studies ${ }^{273}$ have shown that most immigrant women support HIV screening procedures as part of routine medical care.

\section{Recommendations of other groups}

The US Preventive Services Task Force ${ }^{248}$ recommends that clinicians screen all pregnant women, as well as all adolescents and adults at increased risk for HIV (e.g., from countries with HIV prevalence $>1 \%$ ). The Centers for Disease Control and Prevention ${ }^{259}$ recommend screening tests for all sexually active adolescents and adults (15-64 years), citing substantial individual and population benefits of early detection. The UK guidelines ${ }^{253}$ recommend HIV testing for all who present to genitourinary clinics. Our recommendations highlight the importance of routine HIV screening for high-risk immigrant groups.

\section{Take-home messages}

- Immigrants and refugees from countries where HIV is prevalent $(>1 \%)$ are vulnerable because of the high HIV prevalence in their home countries and because of high levels of HIV-related stigma.

- Stigma and discrimination related to HIV are associated with avoidance of or delays in seeking HIV testing, delays in disclosure of seropositive status to partners and practitioners, and postponement or rejection of treatment.

- The effects of HIV-related stigma are magnified among socially vulnerable minority groups.

- Providing information about HIV testing options and effectiveness of treatment can improve the likelihood of testing and acceptance of care.

For the complete evidence review for HIV in immigrant populations, see Appendix 6, available at www.cmaj.ca/lookup /suppl/doi:10.1503/cmaj.090313/-/DC1. 


\section{Hepatitis C}

Chronic infection with hepatitis $\mathrm{C}$ virus affects an estimated 170 million people worldwide. ${ }^{274}$ The chronic form of infection develops in about $70 \%$ of those who have had acute infection. Cirrhosis develops in $20 \%$ of individuals with chronic infection and hepatocellular carcinoma in $1 \%-5 \%$ during the two decades following the initial infection. ${ }^{274}$ Canada has a low burden of hepatitis $\mathrm{C}$, with an estimated seroprevalence of about $0.8 \% .{ }^{275}$ The majority of chronic hepatitis $\mathrm{C}$ virus infections in Canada occur in individuals who have previously or are currently injecting drugs. However, an estimated $20 \%$ of cases occur in immigrants, ${ }^{275}$ and immigrant populations have increased mortality due to viral hepatitis..$^{10,276}$ There is no effective vaccination to prevent acquisition of hepatitis $\mathrm{C}$ virus infection, but the virus can be detected, and treatment regimens are moderately successful in eradicating chronic infections. The treatment regimen averages $24-48$ weeks and is often difficult to tolerate. ${ }^{277,278}$ Emerging data on combination therapies with protease inhibitors have shown substantially improved efficacy with shorter duration of treatments. ${ }^{279}$ We conducted an evidence review to determine the benefits and harms of screening for hepatitis $\mathrm{C}$ virus infection in immigrant populations. The recommendations of the Canadian Collaboration for Immigrant and Refugee Health on screening for hepatitis $\mathrm{C}$ are outlined in Box 9A.

\section{Methods}

We used the 14-step method developed by the Canadian Collaboration for Immigrant and Refugee Health ${ }^{16}$ (summarized in section 3 of this article, above). We considered the epidemiology of hepatitis $\mathrm{C}$ in immigrant populations and defined clinical preventive actions (intervention), outcomes and key clinical questions. We searched MEDLINE (Ovid), MEDLINE InProcess, Embase, CINAHL and the Cochrane Library from 1950 to Jan. 28, 2010, for studies pertinent to immigrants and from Jan. 1, 1997, to Jan. 28, 2010, for studies pertinent to the general population. Detailed methods, search terms, case studies and clinical considerations can be found in the complete evidence review for hepatitis C (Appendix 7, available at www.cmaj.ca/lookup/suppl/doi:10.1503/cmaj $.090313 / / / D C 1)$.

\section{Results}

In the search for systematic reviews and guidelines for hepatitis $\mathrm{C}$ in immigrants, we identified 31 records, but none met our eligibility criteria. In the search for systematic reviews and guidelines for hepatitis $\mathrm{C}$ in the general population, we identified 4714 articles, of which 24 met the eligibility criteria. In addition, a search for articles relevant to hepatitis $\mathrm{C}$ and immigrants yielded 250 articles, of which 50 were relevant and addressed the following areas: epidemiology and knowledge of and compliance with screening and treatment in the immigrant population.

\section{What is the burden of hepatitis $C$ in immigrant populations?}

Canada is a low-prevalence country for chronic infection with hepatitis $\mathrm{C}$ virus. In Canada, deaths from non-A, non-B hepatitis (the majority of which is presumed to be due to chronic hepatitis $\mathrm{C}$ virus infection) have increased 3.4-fold (from 0.12 to 0.41 per 100000 ) over the past 30 years, and the incidence of infection has increased 2.2-fold during the same period. ${ }^{163,164,275}$ These increases are thought to be due to the uncontrolled epidemic among injection drug users and the importation of virus by immigrants from countries where chronic hepatitis $\mathrm{C}$ virus infection is endemic. ${ }^{275}$ The seroprevalence of chronic infection in the immigrant population is estimated to be about $3 \%$ (range $0.1 \%-18 \%$ for various global regions of origin) ${ }^{280-282}$ and likely reflects rates in the countries of origin (Figure 9A)..$^{283,284}$ According to a recent Canadian study, mortality from viral hepatitis was 1.8- to 3.8-fold greater and mortality from hepatocellular carcinomas was 2.2- to 4.9-fold greater among immigrants than in the Canadian-born population..$^{10}$ It is unclear what proportion of deaths due to viral hepatitis and hepatocellular carcinomas

\section{Box 9A: Recommendations from the Canadian Collaboration for Immigrant and Refugee Health: hepatitis $\mathbf{C}$ virus}

Screen for antibody to hepatitis $C$ virus in all immigrants and refugees from regions with an expected prevalence of disease $\geq 3 \%$. If the result is positive, refer to a colleague with expertise in managing patients with hepatitis $C$ virus infection.

\section{Basis of recommendation}

\section{Balance of benefits and harms}

The prevalence of chronic hepatitis $C$ virus infection is higher among immigrants than in the general Canadian-born population (about $3 \%$ v. $0.8 \%$ ). Immigrants are also at increased risk of death from viral hepatitis and hepatocellular carcinoma, a third of which is likely due to chronic hepatitis $C$ virus infection. Treatment with pegylated interferon and ribavirin (standard of care) achieves a higher sustained virologic response than interferon plus ribavirin (50\% v. $38 \%$; relative risk $0.80,95 \%$ confidence interval $[\mathrm{Cl}]$ $0.74-0.88)$. Persons with cirrhosis due to chronic hepatitis $C$ virus infection who did not achieve sustained virologic response had higher rates of hepatocellular carcinoma (hazard ratio [HR] 2.59, 95\% Cl 1.13-5.97) and liver-related mortality (HR 6.97, 95\% Cl 1.71-28.42) than those who did achieve sustained virologic response. Harms included multiple adverse effects of treatments, the most common being psychiatric symptoms and severe anemia.

\section{Quality of evidence}

Moderate

Values and preferences

The committee attributed more value to the diagnosis and prevention of serious complications from hepatitis $\mathrm{C}$ than to the cost and risk of multiple adverse effects of treatments. 
are attributable to chronic infection with hepatitis $\mathrm{C}$ virus in the immigrant population, but it may be as high as $30 \%$. This figure is extrapolated from the fact that hepatitis B and hepatitis C account for $80 \%-90 \%$ of all hepatocellular carcinomas worldwide and that $30 \%$ of all cases are attributable to chronic hepatitis $\mathrm{C}$ virus infection. . $^{276}$

The primary mode of transmission of hepatitis $\mathrm{C}$ virus is percutaneous; sexual or perinatal transmission is much less frequent. As a result, injection drug users represent the group at greatest risk for hepatitis $\mathrm{C}$ in Canada. Immigrants are an important unrecognized risk group for chronic hepatitis $\mathrm{C}$ infection in Canada, but they are likely to have acquired their infection through unsafe health care-related injections or through other medical equipment, unscreened blood products or surgical procedures in their countries of origin. It is estimated that up to $40 \%$ of chronic infections globally are acquired through unsafe injections. ${ }^{285}$ The proportion of immigrants ineligible for treatment because of comorbidities such as psychiatric illness, substance abuse or medical conditions may therefore not be as high as for other at-risk populations such as injection drug users. More than $70 \%$ of the chronic infections in North America are due to genotype 1, whereas in certain countries elsewhere in the world, other genotypes may predominate (e.g., genotype 4 in Egypt and genotype 3 in Pakistan). Immigrants are more likely to have concurrent chronic hepatitis B virus infection or HIV infection, and coinfection will increase the risk and rate of liver fibrosis associated with chronic hepatitis $\mathrm{C}$ virus infection. ${ }^{286}$

\section{Does screening for hepatitis $\mathrm{C}$ virus decrease morbidity?}

\section{Screening tests}

Widely available third-generation serologic enzyme immuno- assays to detect anti-hepatitis $\mathrm{C}$ virus antibodies are highly sensitive (97\%) and specific (99\%). False-positive results occur in populations where the prevalence of hepatitis $\mathrm{C}$ is low, and false-negative results may occur in the setting of severe immunosuppression, such as in patients with HIV, hypogammaglobulinemia or agammaglobulinemia; those who have undergone solid organ transplantation; or those receiving hemodialysis. ${ }^{278}$ If the result is positive, a nucleic acid test to detect hepatitis C RNA (qualitative or quantitative) should be performed to confirm the presence of circulating virus.

\section{Relative benefits and harms of treatment}

The current standard for treatment is combination therapy with pegylated interferon and ribavirin, which achieves an overall sustained virologic response of about $50 \%$ in all patients ${ }^{278,287-290}$ (Table 9A). ${ }^{290,291}$ Sustained virologic response has been associated with improved clinical outcomes in patients with cirrhosis due to chronic hepatitis $\mathrm{C}$ virus infection. In this population, those who did not achieve a sustained virologic response had higher rates of hepatocellular carcinoma (hazard ratio [HR] 2.59, 95\% confidence interval [CI] 1.13-5.97) and liver-related mortality (HR 6.97, CI 1.7128.42) than those who achieved sustained virologic response. ${ }^{292-294}$ The response rate, dosage of the medications and duration of treatment (ranging from 24 to 48 months) are determined by the viral genotype. For infection with genotype 1 virus, treatment response ranges from $42 \%$ to $46 \%$ and requires 48 weeks of treatment. The response is better for those with genotype 2 (74\%) and genotype 3 (69\%), and therapy for these genotypes is of shorter duration (usually 24 weeks). ${ }^{27,288-290,295,296}$ For other genotypes $(4,5,6,7,8)$, the results are less well defined: they appear to be better than for genotype 1 but not as good as for genotypes 2 and 3.29,298 The treatment is associated with numerous adverse effects, and $10 \%$ to $14 \%$ of patients discontinue therapy because of an

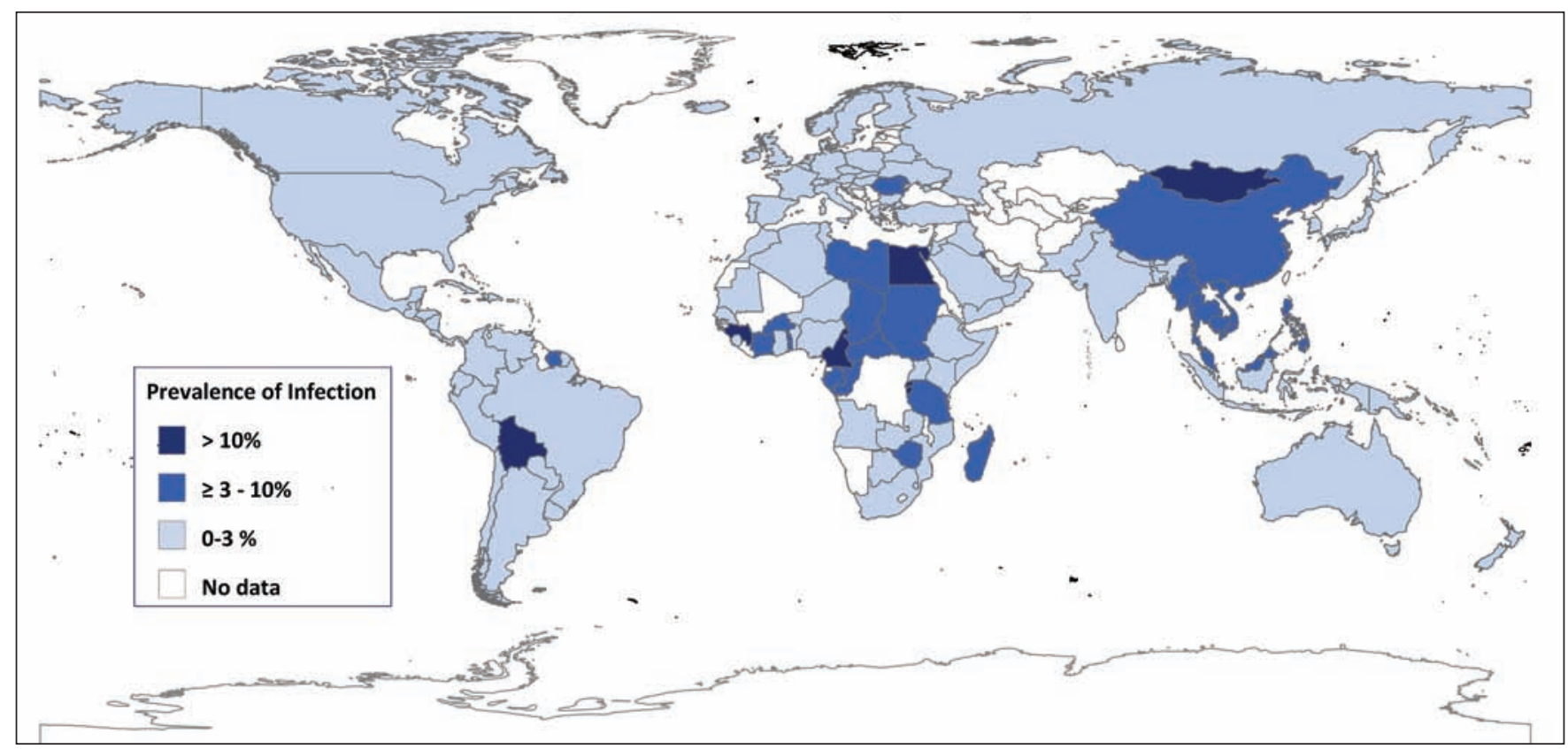

Figure 9A: Estimated worldwide prevalence of hepatitis C, 1999. Based on data from the World Health Organization. ${ }^{284}$ 
adverse event, most commonly psychiatric symptoms or severe anemia. ${ }^{278,299}$ Persons with advanced cirrhosis are less likely to have a response to treatment, which highlights the importance of initiating treatment before advanced liver disease develops. ${ }^{300,301}$ Recent results for combination regimens with protease inhibitors and standard therapy have shown substantially improved efficacy $(70 \%$ v. $50 \%)$ for those with genotype 1 , as well as shorter treatment durations. ${ }^{279,302}$ These new treatment options will likely change the standard of therapy in the near future, making screening and appropriately timed treatment an important strategy for controlling the burden of chronic hepatitis $\mathrm{C}$ virus infection. Management during therapy often requires a multidisciplinary approach, and all patients found to be positive should be referred to a health care professional with expertise in managing hepatitis $\mathrm{C}$ virus infection.

In most studies, screening has been found to be costeffective only when the prevalence of hepatitis $\mathrm{C}$ is high (>10\%) because of the relatively poor efficacy of treatment (overall 50\%), the high adverse-effect profile and the high proportion of persons ineligible for treatment because of underlying comorbidities such as substance abuse, psychiatric illness or medical diseases $(37 \%){ }^{303}$ Plunkett and Grobman ${ }^{304}$ found that screening pregnant women was not cost-effective. They assumed a $1 \%$ seroprevalence of hepatitis $\mathrm{C}$ virus and 48 weeks of treatment with pegylated interferon and ribavirin, but they calculated only direct costs. Singer and Younossi ${ }^{305}$ also found that screening for hepatitis $\mathrm{C}$ virus in the general US population was not cost-effective. They assumed that the seroprevalence was $3 \%$, that only $20 \%$ of individuals found to be positive would be given treatment (because of underlying comorbidities), that the response rate (to interferon and ribavirin) in patients with genotype 1 would be $37 \%$ and that $72 \%$ of all patients would have genotype 1 . In a sensitivity analysis, however, they found that if $50 \%$ of individuals positive for hepatitis $\mathrm{C}$ virus started treatment, then screening would be cost-effective at a seroprevalence of $3 \% .^{304,305}$ Certain ethnic groups, such as Southeast Asians, have a better response to therapy than other ethnic groups. ${ }^{46}$

In summary, we recommend routine screening for immigrant populations in whom the estimated prevalence of chronic hepatitis $\mathrm{C}$ virus infection is $3 \%$ or higher, given their increased risk of death from viral hepatitis and hepatocellular carcinoma, the more favourable response to treatment before development of cirrhosis (which favours early detection), the likelihood that immigrants are more likely to be eligible for treatment and the likelihood that certain immigrant groups can be expected to respond more favourably to treatment than the general Canadian population.

\section{Clinical considerations}

\section{What are the potential implementation issues?}

Few studies have evaluated immigrant populations' knowledge of the importance of hepatitis $\mathrm{C}$, its consequences and the risk factors for transmission. The data suggest that knowledge of hepatitis $\mathrm{C}$ is generally low and that it is significantly lower for immigrants than for nonimmigrants. ${ }^{306,307}$ Predictors of better knowledge are higher levels of education, employment and being highly accultured. ${ }^{307} \mathrm{We}$ found no data on the

Table 9A: Summary of findings for comparison of pegylated interferon plus ribavirin compared with interferon plus ribavirin for treatment of chronic hepatitis C virus infection

Patient or population: Patients with chronic hepatitis $C$ virus infection

Settings: Multiple countries (including Italy, Egypt, Japan, Taiwan, Germany, Saudi Arabia, Belgium)

Intervention: Pegylated interferon plus ribavirin

Comparison: Interferon plus ribavirin

Sources: Shepherd J, Brodin H, Cave C, et al. Pegylated interferon alpha-2a and -2b in combination with ribavirin in the treatment of chronic hepatitis C: a systematic review and economic evaluation. Health Technol Assess 2004;8:iii-iv, 1-125. ${ }^{291}$ Simin M, Brok J, Stimac $D$, et al. Cochrane systematic review: pegylated interferon plus ribavirin vs. interferon plus ribavirin for chronic hepatitis $C$. Aliment Pharmacol Ther 2007;25:1153-62..$^{290}$

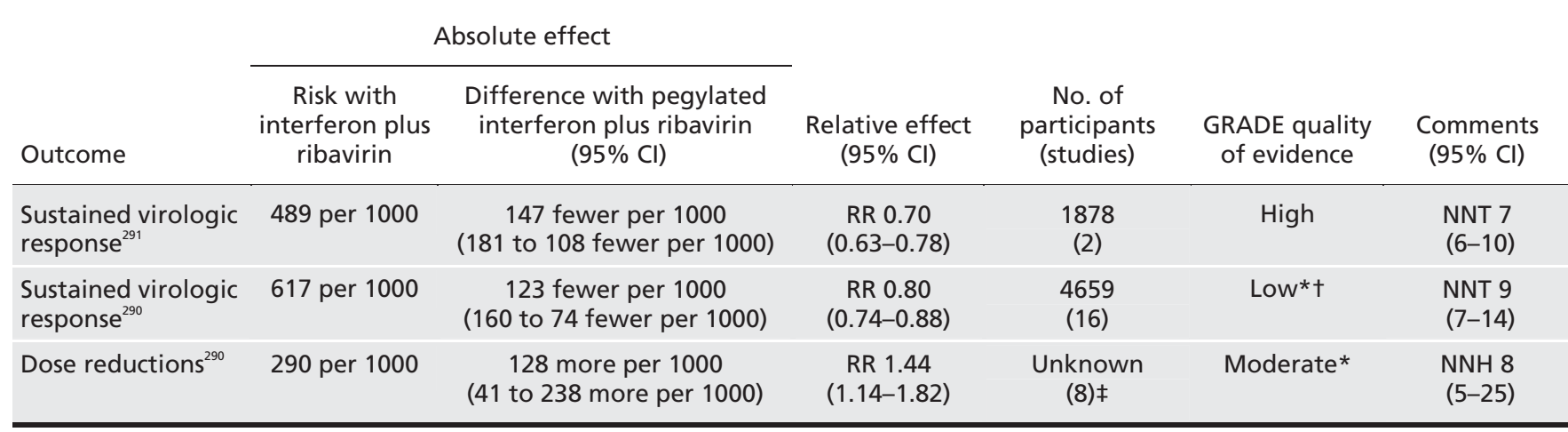

Note: $\mathrm{Cl}=$ confidence interval; GRADE = Grading of Recommendations Assessment, Development and Evaluation; NNH = number needed to harm; NNT = number needed to treat; $\mathrm{RR}=$ risk ratio.

* Lack of blinding, inadequate allocation concealment

†Significant heterogeity $\left(I^{2}=56 \%, p=0.003\right)$

$\ddagger$ Actual number of participants not reported; only percentages and RR values provided. 
proportion of immigrants who accept screening for hepatitis $\mathrm{C}$ virus or their compliance with treatment of infection. In addition to potential patient barriers to the uptake of screening, there are likely important barriers that prevent primary care practitioners from recommending screening. More than half of primary care physicians have little experience in treating patients who test positive for infection, have a poor understanding of the natural history of the disease and incorrectly identify risk factors for infection. ${ }^{308}$ A large proportion (60\%) of primary care physicians do not routinely ask patients about risk factors for hepatitis $\mathrm{C}$ on an initial visit. ${ }^{309,310}$ Of those who did identify higher-risk patients, only $50 \%$ to $75 \%$ ordered the appropriate diagnostic tests and correctly interpreted the results. ${ }^{311}$

\section{Recommendations of other groups}

The US Preventive Services Task Force does not recommend screening for hepatitis $\mathrm{C}$ in the general US population (seroprevalence of about $1.8 \%$ ) but rather recommends screening high-risk groups such as injection drug users. ${ }^{312}$ Neither the recent Canadian or US guidelines for managing hepatitis $\mathrm{C}$ identify immigrants as an at-risk group that should be targeted for screening. ${ }^{277,278}$ Our guidelines high- light the potential benefits of routine hepatitis $\mathrm{C}$ screening for at-risk immigrant groups.

\section{Take-home messages}

- About 3\% of immigrants are infected with chronic hepatitis $\mathrm{C}$ virus (up to $18 \%$ in certain populations), and a large proportion of these will likely be eligible for treatment.

- The majority of immigrants acquire infection through unsafe injections or other medical procedures in their countries of origin, rather than through injection drug use.

- Immigrants are more likely to have concurrent infection (e.g., with hepatitis B or HIV), which increases the risk of progression of chronic hepatitis $\mathrm{C}$ virus-associated disease.

- Screening individuals for chronic hepatitis $C$ virus infection and offering treatment before development of cirrhosis is important, because sustained virologic response is higher and treatment is better tolerated in the absence of cirrhosis.

For the complete evidence review for hepatitis $\mathrm{C}$ in immigrant populations, see Appendix 7, available at www.cmaj.ca/lookup /suppl/doi:10.1503/cmaj.090313/-/DC1. 


\section{Intestinal parasites: Strongyloides and Schistosoma}

About one-third of the world's population is infected with intestinal parasites, ${ }^{47}$ and most of these infections are sustained through cycles of repeated exposure from the environment. When populations emigrate from parts of the world where intestinal parasites are endemic and resettle in countries where they do not exist, most infections will clear without treatment within a few years after immigration. Two intestinal parasites - Strongyloides and Schistosoma - are notable exceptions in that they may persist for decades as subclinical infections or as low-grade disease with nonspecific clinical manifestations. In the presence of immunosuppression, strongyloidiasis can evolve rapidly into life-threatening disseminated disease, whereas chronic schistosomiasis can result in complications causing future morbidity and death. We conducted an evidence review to guide primary care practitioners in the early detection and treatment of strongyloidiasis and schistosomiasis for newly arriving refugees. The recommendations of the Canadian Collaboration for Immigrant and Refugee Health on screening for and treatment of these parasites are outlined in Box 10A.

\section{Methods}

We used the 14-step method developed by the Canadian Collaboration for Immigrant and Refugee Health ${ }^{16}$ (summarized in section 3 of this article, above). We considered the epidemiology of intestinal parasites in immigrant populations and defined clinical preventive actions (interventions), outcomes and key clincial questions. We search MEDLINE, Embase, the Database of Abstracts of Reviews of Effectiveness, the HTA Database, the Cochrane Library and other sources from Jan. 1, 1980, to Jan. 1, 2010. Detailed methods, search terms, case studies, clinical considerations and research recommendations can be found in the complete evidence review for intestinal parasites (Appendix 8, available at www .cmaj.ca/lookup/suppl/doi:10.1503/cmaj.090313/-/DC1).

\section{Results}

Our electronic search strategy for systematic reviews yielded 5039 articles. We identified an additional 150 articles through an open-ended search. We reviewed the titles of all 5189 articles, and evaluated 445 abstracts for potential relevance, eliminating 437 because they did not fulfill our inclusion criteria. Of the remaining eight manuscripts, five pertained to the diagnosis of strongyloidiasis ${ }^{313-316}$ or schistosomiasis, ${ }^{317}$ and the remaining three pertained to treatment of these conditions. ${ }^{318-320}$ Although our search did not identify any systematic reviews on the diagnosis of either parasite or the treatment of strongyloidiasis, we found a systematic review pertaining to the treatment of schistosomiasis. ${ }^{319}$ Furthermore, we found two major guidelines pertaining to the evaluation and management of parasitic infections in newly arriving refugees..$^{23,321}$

Box 10A: Recommendations from the Canadian Collaboration for Immigrant and Refugee Health: intestinal parasites

\section{Strongyloides}

Screen refugees newly arriving from Southeast Asia and Africa with serologic tests for Strongyloides, and treat, if positive, with ivermectin (first-line therapy) or albendazole (if there are contraindications to ivermectin).

\section{Basis of recommendation}

\section{Balance of benefits and harms}

Strongyloides is estimated to infect 100 million people worldwide. Among immigrant populations, refugees from Southeast Asia and Africa appear to have the highest risk of infection. Subclinical infections or low-grade disease can persist for decades after immigration and in the presence of immunosuppression may transform into life-threatening disseminated disease. Treatment with ivermectin is of short duration, is highly effective (number needed to treat [NNT] 2, $95 \%$ confidence interval $[\mathrm{Cl}] \sim 1$ to 3 ) and has a favourable adverse-effect profile.

\section{Quality of evidence}

Moderate

\section{Values and preferences}

The committee attributed more value to the availability of a highly sensitive and specific serologic test and effective treatment options to prevent potentially life-threatening disseminated disease than to the potential limitations of serologic testing in distinguishing current from remote infection in high-risk newly arriving refugees.

\section{Schistosoma}

Screen refugees newly arriving from Africa with serologic tests for Schistosoma, and treat, if positive, with praziquantel.

\section{Basis of recommendation}

\section{Balance of benefits and harms}

Schistosoma is estimated to infect 200 million people worldwide, of whom approximately $85 \%$ live in Africa. Among immigrant populations, refugees from Africa have the highest risk of infection. Subclinical infections or lowgrade disease can persist for decades after immigration and may cause future morbidity or death. Serologic testing is the most sensitive diagnostic modality currently available. Treatment with praziquantel is of short duration, is highly effective (NNT 4, 95\% Cl 1 to 124) and has a favourable adverse-effect profile.

\section{Quality of evidence}

Moderate

\section{Values and preferences}

The committee attributed more value to the availability of a highly sensitive and specific serologic test and effective treatment to prevent future morbidity or death than to the limitations of serologic testing in distinguishing current from remote infection in high-risk newly arriving refugees. 


\section{What is the burden of strongyloidiasis and schistosomiasis in immigrant populations?}

Quantifying the burden of parasitic infection is challenging because existing studies have not involved systematic or random testing of immigrant populations. Most estimates of burden have been derived from small observational studies and included primarily refugees from selected countries. Furthermore, a significant number of these studies used stool microscopy, a diagnostic test that is known to have limited sensitivity in the detection of each of these parasites.

In the case of strongyloidiasis, existing data have been derived primarily from refugee populations originating from Southeast Asia and Africa (Figure 10A). Studies using stool microscopy have reported prevalence rates between $0.8 \%$ and $4.3 \%,,^{322-327}$ the highest burden being identified in refugees from Southeast Asia. ${ }^{322,327}$ Studies using serologic enzyme immunoassays have reported significantly higher prevalences of infection (between 9\% and 77\%), ${ }^{107,316,328-333}$ the highest burden being identified in refugees from Southeast Asia ${ }^{316,329,330}$ and Africa. ${ }^{328,331,334}$

About $85 \%$ of the global burden of schistosomiasis is believed to occur in Africa. ${ }^{335}$ Studies using stool microscopy to detect Schistosoma in African refugee populations have reported prevalences from $0.4 \%$ to $7 \% .{ }^{323-325,336}$ In contrast, studies using serologic enzyme immunoassays have reported significantly higher prevalences, ${ }^{107,328,329,331-334,337,338}$ ranging from $2.2 \%$ in East African pediatric populations ${ }^{332}$ to $64 \%$ in Sudanese refugees and $73 \%$ in Somalia refugees. ${ }^{331,334}$

\section{Does screening for strongyloidiasis and schistosomiasis decrease morbidity?}

The scope and analytic horizon of existing studies prevent establishment of a direct link between screening for strongyloidiasis or schistosomiasis and an improvement in health outcomes. The association can be derived indirectly, however, since highly sensitive and specific diagnostic tests are currently available to detect each parasite, and effective treatment is known to mitigate the risk of future morbidity or death.

\section{Screening tests}

Stool microscopy for ova and parasites is the only definitive way to confirm the presence of intestinal infection with either Strongyloides or Schistosoma, but this diagnostic modality has suboptimal sensitivity. Although overall sensitivity can be improved by increasing the number of stool specimens examined, the costs associated with this approach can be substantial, and many patients are reluctant to provide multiple specimens. The sensitivity of a single stool examination to detect Strongyloides is estimated at just $30 \%$, but this increases to over $90 \%$ when seven specimens are examined..$^{339}$

By contrast, serologic testing is the most sensitive diagnostic modality to detect Strongyloides and Schistosoma, making such tests ideal screening tools. Although these tests are also quite specific, serologic positivity cannot definitively distinguish current from remote infection. The National Reference Centre for Parasitology in Montréal,

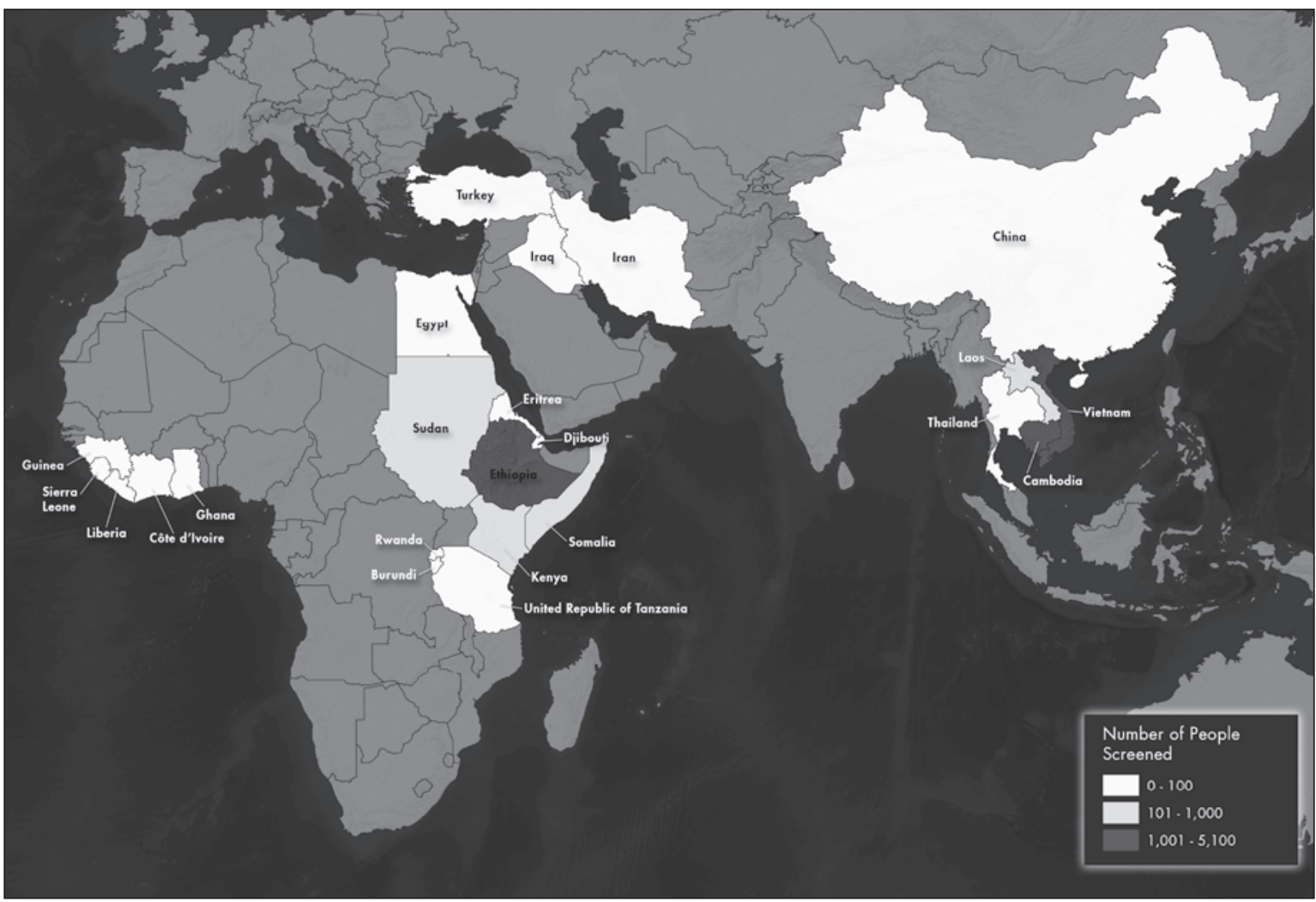

Figure 10A: Number of immigrants and refugees screened for strongyloidiasis by birth country, as identified in our review of the medical literature between 1988 and 2010. 
which performs serologic testing for both parasites in Canada, estimates that its enzyme immunoassays have $100 \%$ sensitivity and $88 \%$ specificity for Strongyloides stercoralis and $96 \%$ sensitivity and $82 \%$ specificity for Schistosoma mansoni. Several studies have reported levels of antibodies to Strongyloides declining after treatment, ${ }^{48,340-343}$ which suggests that serologic positivity is indicative of current infection; however, this has not been a universal finding. ${ }^{315}$ Levels of antibodies to Schistosoma do not appear to decline after treatment. ${ }^{317}$ Nonetheless, given the potential for these infections to persist for decades and to cause potentially lifethreatening disease, it is generally presumed that a positive result on serologic testing for either parasite in high-risk newly arriving refugees (without a history of recent effective treatment) represents current infection.

\section{Relative benefits and harms of treatment}

The detection and subsequent treatment of strongyloidiasis and schistosomiasis can prevent future morbidity and potentially life-threatening complications. A two-day course of ivermectin $(200 \mu \mathrm{g} / \mathrm{kg}$ orally once daily) is the preferred treatment for strongyloidiasis (Table 10A); however, among refugees from areas of the world where Loa loa is endemic, a seven-day course of albendazole (400 mg orally twice daily) should be used. This is because cases of encephalopathy have been reported with use of ivermectin during large-scale treatment campaigns in West and Central Africa, where Loa loa is endemic (see the guidelines of the US Centers for Disease Control and Prevention ${ }^{321}$ for a table of countries where Loa loa is endemic). This rare but potentially serious event may occur in persons who have a high load of Loa loa microfilaria,

Table 10A: Summary of findings for comparison of ivermectin and pyrvinium pamoate for strongyloidiasis

Patient or population: Patients with strongyloidiasis

Setting: Okinawa, Japan

Intervention: Ivermectin

Comparison: Pyrvinium pamoate

Source: Toma H. Comparative studies on the efficacy of three anthelminthics on treatment of human strongyloidiasis in Okinawa, Japan. Southeast Asian J Trop Med Public Health 2000;31:147-51. 320

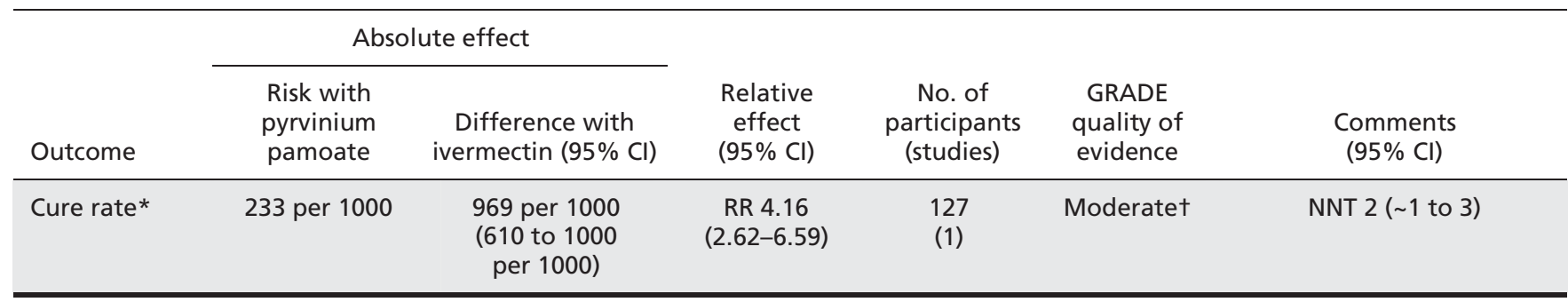

Note: $\mathrm{Cl}$ = confidence interval; GRADE = Grading of Recommendations Assessment, Development and Evaluation; NNT = number need to treat; RR = risk ratio. *Common adverse effects with treatment are pruritis, fever and tenderness of the lymph nodes. Serious or fatal encephalopathy has been reported (rarely) during treatment of patients with loiasis. Therefore, pretreatment assessment for Loa loa infection is recommended for any patient emigrating from areas where such infection is endemic (West and Central Africa).

tNot randomized, no blinding.

Table 10B: Summary of findings for comparison of praziquantel and placebo for treatment of schistosomiasis

Patient or population: Patients with schistosomiasis

Setting: Sudan, Zambia, Burundi, Democratic Republic of Congo, Kenya, Tanzania, Philippines, China, Republic of Congo, Niger

Intervention: Praziquantel

Comparison: Placebo

Source: Kumar V, Gryseels B. Use of praziquantel against schistosomiasis: a review of current status. Int J Antimicrob Agents $1994 ; 4: 313-20 .^{318}$

\begin{tabular}{|c|c|c|c|c|c|c|}
\hline Outcome & \multicolumn{2}{|c|}{ Absolute effect } & $\begin{array}{l}\text { Relative effect } \\
\quad(95 \% \mathrm{Cl})\end{array}$ & $\begin{array}{l}\text { No. of } \\
\text { participants } \\
\text { (studies) }\end{array}$ & $\begin{array}{l}\text { GRADE } \\
\text { quality of } \\
\text { evidence }\end{array}$ & $\begin{array}{c}\text { Comments } \\
(95 \% \mathrm{Cl})\end{array}$ \\
\hline Parasitologic cure & NA & NA & $\begin{array}{c}\text { RR } 28.29 \\
(1.81-441.61)\end{array}$ & $\begin{array}{l}69 \\
(1)\end{array}$ & Moderate* & $\begin{array}{c}\text { NNT } 4 \\
(\sim 1 \text { to } 124)\end{array}$ \\
\hline $\begin{array}{l}\text { Adverse effectst } \\
\text { (1\%-10\%; medium-risk } \\
\text { population) }\end{array}$ & 60 per 1000 & $\begin{array}{l}361 \text { per } 1000 \\
(173 \text { to } 750 \\
\text { per } 1000)\end{array}$ & $\begin{array}{c}\text { RR } 6.01 \\
(2.89-12.5)\end{array}$ & $\begin{array}{l}436 \\
(1)\end{array}$ & NA & NA \\
\hline
\end{tabular}

Note: $\mathrm{Cl}=$ confidence interval; GRADE = Grading of Recommendations Assessment, Development and Evaluation; NA = not applicable; NNT = number need to treat; $\mathrm{RR}=$ risk ratio.

*No details on randomization or blinding

tDizziness, headache, malaise, abdominal pain 
which are rapidly killed by ivermectin. Alternatively, because ivermectin is the most effective treatment option available for strongyloidiasis, practitioners may screen refugees at risk for Loa loa infection with a daytime-collected, thin blood smear for microfilaria and treat with ivermectin if high-level microfilaremia is not identified. For Schistosoma species found in Africa, a one-day course of praziquantel $(40 \mathrm{mg} / \mathrm{kg}$ divided in two doses) is the preferred treatment strategy (Table 10B).

Treatment with ivermectin or praziquantel in patients with underlying neurocysticercosis may lead to an acute inflammatory reaction and could precipitate seizure activity. Therefore, these drugs should not be used in persons with known neurocysticercosis or an unexplained seizure disorder. Otherwise, ivermectin, albendazole and praziquantel each have a generally favourable side-effect profile..$^{344}$

\section{Clinical considerations}

On the basis of this review, we propose routine serologic screening for all newly arriving refugees at high risk for strongyloidiasis and schistosomiasis. With limited data on the burden of these two parasites in nonrefugee immigrant populations, there is currently insufficient evidence to justify routine screening of all nonrefugee populations, even though some high-risk groups may be missed. ${ }^{345}$ Further research is needed to clarify the burden of illness in other immigrant populations.

Practitioners should consider testing foreign-born persons for strongyloidiasis and/or schistosomiasis if they have lived in areas of the world where these parasites are endemic and (i) they have compatible signs and/or symptoms of infection (independent of the time elapsed since their arrival into Canada) and/or (ii) they have evidence of peripheral blood eosinophilia. Clinicians should also be aware that persons infected with the retrovirus human T-lymphotropic virus 1 (HTLV-1) have a modified immune response that complicates the treatment of strongyloidiasis. ${ }^{346}$ Some areas of the world are endemic for both Strongyloides and HTLV-1, and clinicians should therefore consider screening for the retrovirus if a patient (i) tests positive for Strongyloides and originates from an area with high prevalence of HTLV-1 (i.e., South America, the Caribbean, Japan or Africa) and/or (ii) has persistent strongyloidiasis that responds poorly to antiparasitic treatment.

Finally, the studies in this review primarily involved adult refugees, but children are well known to be at risk of infection with intestinal parasites. Consequently, screening newly arriving refugees of all ages from areas of the world endemic for strongyloidiasis and schistosomiasis is suggested. However, treatment with albendazole is not recommended in children under one year of age. Similarly, treatment with ivermectin is not recommended for children weighing less than $15 \mathrm{~kg}$ or less than $90 \mathrm{~cm}$ in length, and praziquantel is not recommended for children under four years of age. Infected children in these circumstances should be referred to a practitioner experienced in the management of intestinal parasites in pediatric populations.

Serologic samples to be tested for Strongyloides and Schistosoma are processed at the National Reference Laboratory for Parasitology in Montréal, so primary care providers should anticipate delays from the time a specimen is collected until test results are received. Furthermore, if patients require treatment for strongyloidiasis with ivermectin or albendazole, these drugs must be obtained through Health Canada's Special Access Programme. Instructions on how to obtain these medications can be found at Health Canada's website. ${ }^{347}$ By contrast, praziquantel can be obtained locally through a physician's prescription.

Serologic testing after treatment for schistosomiasis is not recommended, as the antibodies tend to persist over time. By comparison, several studies have reported declining strongyloides antibody titres 6 to 12 months after successful treatment, ${ }^{48,340-343}$ and the use of serologic testing has been advocated as a marker for clearance of this parasite. Although there is a body of evidence demonstrating this post-treatment effect, ${ }^{48,340-343}$ this finding has not been universally observed. ${ }^{315}$ A practice of post-treatment serologic testing may be considered by practitioners, but at a minimum, all treated individuals should be followed prospectively for clinical signs or symptoms of persistent infection and to ensure that eosinophil counts remain within or return to normal limits within six months of receiving effective treatment. Should patients have persistent symptoms and/or eosinophilia after six months, further investigations - including the option of repeat serologic testing for Strongyloides - should be pursued.

\section{Recommendations of other groups}

Two sets of national guidelines offer recommendations on the diagnosis and management of intestinal parasites in refugee populations. Both the US Centers for Disease Control and Prevention ${ }^{321}$ and the Australasian Society for Infectious Disease $^{23}$ support the use of serologic testing as part of screening for strongyloidiasis and schistosomiasis. Our recommendations focus routine serologic screening on migrant populations known to have high prevalence rates for these two parasitic infections.

\section{Take-home messages}

- Strongyloidiasis and schistosomiasis are parasitic infections that can persist for years to decades and consequently can cause serious morbidity or death long after an immigrant resettles in a new country.

- The burden of strongyloidiasis appears greatest in refugee populations originating from Southeast Asia and Africa, whereas the burden of schistosomiasis is greatest in refugee populations from Africa.

- Detection of strongyloidiasis or schistosomiasis is limited by subclinical infection or low-grade disease and by the suboptimal sensitivity of stool microscopy.

- Serologic testing substantially enhances diagnostic sensitivity.

For the complete evidence review for intestinal parasites in immigrant populations, see Appendix 8, available at www .cmaj.ca/lookup/suppl/doi:10.1503/cmaj.090313/-/DC1. 


\section{Malaria}

In 2006, worldwide, there were 250 million cases of malaria, leading to one million deaths. ${ }^{49}$ A large proportion of Canada's new immigrants and refugees come from malariaendemic countries (Figure 11A). ${ }^{100,348}$ The symptoms of malaria are nonspecific; ${ }^{349}$ they include fever and a constellation of other findings ranging from mild illness to fulminant organ failure and death. Plasmodium falciparum is the most virulent causative species, with an overall $1 \%$ case fatality rate. ${ }^{350}$ This rate increases to $10 \%-20 \%$ or higher for those with severe disease, defined by high parasitemia or end-organ damage. ${ }^{348,350}$ Most imported cases of malaria, particularly those due to $P$. falciparum, present within three months of leaving an endemic area. ${ }^{351-353}$ Canadian health care providers may have difficulties in diagnosing and treating malaria because of the rarity of the disease and unfamiliarity with laboratory diagnosis and clinical management. ${ }^{50,350}$ As well, drugs for treatment of malaria may not be readily accessible in pharmacies across the country. These delays, which may be compounded by migrants' unfamiliarity with the Canadian health care system, can lead to severe disease and even death. ${ }^{351,353-357}$ In addition, malarial illness may interfere with the migrant's successful integration into the host community because of issues such as physical incapacity and added financial stress. We undertook this review to determine whether Canadian primary care physicians should routinely screen for malaria in asymptomatic adult and child migrants from low- to middleincome countries. The recommendations of the Canadian Collaboration for Immigrant and Refugee Health on malaria are outlined in Box 11A.

\section{Methods}

We used the 14-step method developed by the Canadian Collaboration for Immigrant and Refugee Health ${ }^{16}$ (summarized in section 3 of this article, above). We considered the epidemiology of malaria in immigrant populations and defined clinical preventive actions (interventions), outcomes and key clinical questions. We searched MEDLINE, Embase, CINAHL, HealthSTAR, the Cochrane Library and other sources from Jan. 1, 1996, to Dec. 31, 2010. Detailed methods, search terms, case studies and clinical considerations can be found in the complete evidence review for malaria (Appendix 9, available at www.cmaj.ca/lookup/suppl/doi:10.1503 /cmaj.090313/-/DC1).

\section{Results}

The initial searches yielded 1421 articles, of which 101 were selected for detailed appraisal. Initial searches for documents about screening for malaria in immigrants and refugees generated many retrospective and prospective reviews of screening in asymptomatic migrants, several of which related to refugees. ${ }^{358-361}$ Some of these articles provided recommendations on screening for malaria in migrants from areas where the disease is endemic; however, none reported use of a sys- tematic review methodology. We found no randomized controlled trials investigating the effect of routine screening of asymptomatic individuals on morbidity and mortality related to malaria. The search for articles about treatment of malaria yielded 247 records, including two systematic reviews. ${ }^{362,363}$

\section{What is the burden of malaria in immigrant populations?}

There are limited data on the screening of asymptomatic migrants. Since 2009, Citizenship and Immigration Canada has screened refugees from East Africa, using rapid diagnostic testing for the antigen 48-72 hours before departure and treating all people with a positive result. During 2009 and the first nine months of 2010, a total of 3384 Canadian-bound refugees were screened, of whom $3.7 \%$ tested positive (unpublished data, Citizenship and Immigration Canada). The prevalence of seropositivity in Canadian-bound refugees from other areas and in nonrefugee migrants is unknown, as these

\section{Box 11A: Recommendations from the Canadian Collaboration for Immigrant and Refugee Health: malaria}

Do not conduct routine screening for malaria.

Be alert for symptomatic malaria in migrants who have lived or travelled in malaria-endemic regions within the previous three months, particularly in the context of fever or migration from sub-Saharan Africa, and perform timely diagnostic inquiry and testing (rapid diagnostic testing and thick and thin malaria smears).

\section{Basis of recommendation}

\section{Balance of benefits and harms}

Individuals from malaria-endemic regions, particularly migrants from sub-Saharan Africa, remain vulnerable to acute Plasmodium falciparum malaria for the first three months after arrival. Clinical trials demonstrating the value of routine screening for asymptomatic malaria are lacking, prevalence data remain poor, the performance characteristics of malaria screening tests in asymptomatic individuals are uncertain, and local transmission of malaria is nonexistent in Canada. Thus, the focus of recommendations is on timely diagnosis and treatment of symptomatic malaria, where medications are effective and harms from adverse effects are minimal.

\section{Quality of evidence}

Low

\section{Values and preferences}

The committee attributed more value to avoiding burden and cost from routine screening in the absence of clear evidence of prevalence of $P$. falciparum and uncertainty of performance of screening tests in asymptomatic individuals and determined that malaria was best addressed by primary care practitioners remaining alert for signs and symptoms of the disease and performing timely clinical diagnostic inquiry and treatment of symptomatic individuals. 
groups are not screened before departure. Published reports of screening protocols for new migrants have indicated variable prevalence of malaria. Specifically, prevalence ranges from $6.8 \%$ to $64 \%$ among African migrants, most of whom are refugees. The prevalence among people from other malariaendemic areas is much lower. ${ }^{32,352,358-360,364,365} \mathrm{~A}$ limited number of follow-up studies suggest that the risk of development of symptomatic malaria after screening is $20 \%$ to $40 \%$. $358-360,365$

The burden of symptomatic malaria among Canadian migrants is difficult to ascertain. The Public Health Agency of Canada receives reports of 350 to 1000 imported malaria cases per year, but the reason for travel in these cases is not reported. Such information is available, through the Canadian Malaria Network, only for cases of malaria requiring parenteral therapy, with 150 cases being reported from June 2001 through January 2010. Of these, 31 cases (20.7\%) occurred in new migrants. Sixty-five percent of these (20/31) were children, whereas the overall percentage of pediatric cases was much lower (26.7\% [40/150]). All but one migrant case originated from Africa, the exception occurring in a recent Karen refugee (from Burma). ${ }^{353}$ In Alberta, new migrants accounted for $20.7 \%$ (79/382) of malaria cases over 10 years (unpublished information, Alberta Health and Wellness). Data compiled from three Canadian tropical medicine clinics (GeoSentinel Surveillance Network Sites in Montréal, Ottawa and Toronto), reporting mostly illness in adults, for January 2006 through October 2010, found that migrants accounted for $15.1 \%(22 / 146)$ of malaria cases, with children accounting for $6.8 \%(10 / 146)$ of all cases and $27.3 \%$ $(6 / 22)$ of cases involving migrants; overall, $60 \%$ of childhood cases occurred in migrants (D. Freedman, GeoSentinel Surveillance Network, Birmingham, Ala.: personal communication, 2010). In the United States, malaria in migrants accounted for $7.9 \%(362 / 4597)$ of all malaria cases from 2005 to 2007. Between 2007 and 2008, children were disproportionately represented among US migrant cases, accounting for $66.3 \%$ of malaria cases in that population, compared with about $20 \%$ of all malaria $\operatorname{cases}^{351,357}$ (P. Arguin, Surveillance Reports to Domestic Malaria Branch, Centers for Disease Control and Prevention. Atlanta, Ga.: personal communication, 2010). Most nonsurveillance studies report on the movements of refugees or case series in specialty clinics. ${ }^{352,366,367}$ In one pediatric case series from British Columbia, $28.6 \%$ of cases (over 17 years) occurred in immigrants. ${ }^{368}$ These data confirm the occurrence of symptomatic malaria, including severe malaria, in new migrants, including those to Canada, and highlight the disproportionate burden of the disease in migrant children.

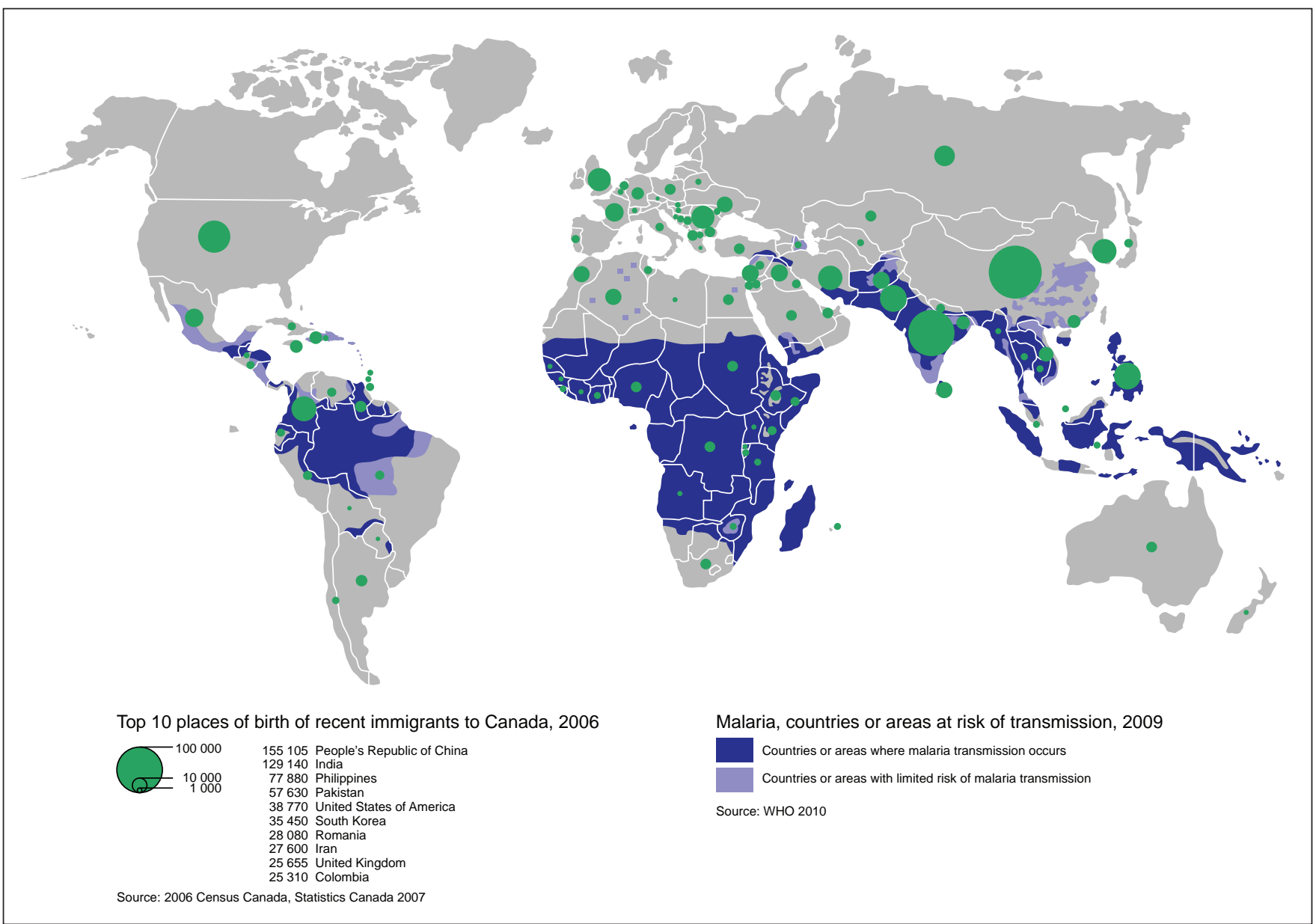

Figure 11A: Map of migrant source countries with overlay of malaria endemicity. Based on data from the 2006 Census of Canada ${ }^{100}$ and the World Health Organization. ${ }^{348}$ 


\section{Does screening for malaria decrease morbidity and mortality?}

\section{Screening tests}

Microscopic diagnosis with Giemsa-stained thick and thin blood smears has traditionally been the gold standard for diagnosing malaria and determining the level of parasitemia. These tests are inexpensive and ubiquitous, ${ }^{369}$ but time-consuming, and they rely on substantial expertise that is not readily available in laboratories outside of malariaendemic areas. Rapid diagnostic tests detect antigens from lysed parasite-infected red blood cells, providing results within 5-20 minutes. These tests have the advantage of portability and ease of use. The usual lower limit of detection is 100 parasites $/ \mu \mathrm{L}$, with sensitivity declining at lower levels of parasitemia. ${ }^{369}$ A nonsystematic review concluded that the sensitivity of rapid diagnostic tests for $P$. falciparum was $95.3 \%$ (95\% confidence interval [CI] 93\%97\%); this dropped to $89.2 \%$ (95\% CI 75\%-97\%) for 100 500 parasites $/ \mu \mathrm{L}$. The specificity for $P$. falciparum was 94.2\% (95\% CI 93\%-95\%). However, the ability to detect $P$. vivax malaria was not as robust, with a sensitivity of $68.9 \%$ (95\% CI 66\%-72\%) and specificity $99.8 \%$ (95\% CI 99\%-100\%). ${ }^{370}$ There is some uncertainty about the performance of rapid diagnostic tests in asymptomatic individuals. Polymerase chain reaction-based assays are also used to diagnose malaria and have lower detection limits. ${ }^{358,371,372}$ However, this significant improvement in detection levels is offset by the cost, institutional support, accessibility and time required for test results. ${ }^{371}$

In Canada, there are many regions where laboratories are unaccustomed to diagnosing malaria by microscopy. The technology, convenience and affordability make rapid diagnostic tests a suitable alternative for Canadian health laboratories.

\section{Relative benefits and harms of treatment}

Data from predeparture treatment protocols for asymptomatic African refugees to Australia and the United States indicate that there has been a reduction in malaria cases among migrants. ${ }^{366,367,373}$ Of concern, however, are reports of treatment failures, often thought to be due to problems with completion of therapy or delays in departure following therapy..$^{37,375}$ The efficacy of treating symptomatic malaria is well documented. ${ }^{348,350}$ The World Health Organization recommends artemisinin combination therapy as first-line treatment for P. falciparum malaria. ${ }^{348}$ However, these drugs are not licensed or available in Canada, where atovaquone and proguanil or quinine plus doxycycline (clindamycin in pregnancy or under the age of eight years) are recommended. Diagnosis and management for symptomatic individuals should follow the Canadian malaria guidelines. ${ }^{350}$

In summary, although detecting and treating asymptomatic parasitemia may decrease morbidity and mortality from malaria, there is currently a paucity of clinical trials demonstrating the benefits and harms of routine screening and insufficient data to establish the true risk in migrants to Canada.

\section{Clinical considerations}

In 2009, Citizenship and Immigration Canada began screening for malaria, by means of rapid diagnostic testing, all refugees destined to Canada from East Africa; screening is performed 48-72 hours before departure. When the result of such testing is positive, treatment is provided with a full course of artemether and lumefantrine (Coartem). For lactating women and for children under $5 \mathrm{~kg}$, a combination of artesunate and amodiaquine is used. For pregnant women, quinine is used. Australia follows the same protocol as Canada, whereas the United States provides presumptive treatment with artemether and lumefantrine to all persons except those who are pregnant, lactating or under $5 \mathrm{~kg}$. For the latter groups, rapid diagnostic testing is provided, and only those with a positive result are treated.

It is important to remember that the majority of individuals migrating from areas of malaria risk, particularly sub-Saharan Africa, are not refugees from East Africa and will not have received predeparture malaria screening. As well, malaria may develop even in those who have undergone screening, including those who have received therapy, especially in the first three months after migration. Many new migrants to Canada are unfamiliar with the health care system, and those from malariaendemic countries may be accustomed to having access to malaria therapy without visiting a physician or obtaining a prescription, and the perceived cost of medical care may be a barrier to receiving care. ${ }^{376-378}$ As well, migrants may be interacting with health care providers who are unfamiliar with diagnosis and management of the disease..$^{50}$ Depending on the area of relocation, effective drugs for treating malaria may not be readily available. These factors may delay detection and treatment, thereby increasing the risk of severe malaria disease. ${ }^{353}$

Numerous studies have documented the increased risk of malaria, including severe malaria, in migrants and their children who return to visit friends and family in malaria-endemic countries. ${ }^{351,353,354,357,379-381}$ Practitioners can take the opportunity to introduce the concept of future malaria risk and the need to seek pretravel advice for recommendations on preventing and managing malaria.

\section{Recommendations of other groups}

Canada has guidelines for prevention and treatment of malaria, ${ }^{350}$ but they do not address the topic of screening immigrants and refugees. In the United States, the Centers for Disease Control and Prevention have technical instructions addressing predeparture treatment of refugees, ${ }^{382}$ which recommend postarrival presumptive therapy for those who missed predeparture therapy. In Australia, there are guidelines for screening refugees from Africa who have resided in or travelled through a malaria-endemic region. The Australian guidelines advocate screening (with both thick and thin smear and rapid diagnostic testing) and treatment instituted by or in consultation with a specialist infectious disease service. ${ }^{23}$ Our guidelines highlight the risk of malaria but also the limitations of current evidence to support routine screening of asymptomatic migrants. 


\section{Take-home messages}

- Routine screening is not recommended, but clinicians should be vigilant for symptoms of malaria.

- Migrants who have lived or travelled in malaria-endemic areas are vulnerable to acute malaria, particularly within the first three months after arrival.

- The symptoms of this disease (malaise, myalgia, headache and fever) are nonspecific, and primary care practitioners may not readily recognize them as symptoms of malaria.
- Delays in the diagnosis and treatment of $P$. falciparum infection may lead to severe disease and even death.

- Improved surveillance for malaria is needed in Canada, as well as more research related to the utility of screening immigrants and refugees for this disease.

For the complete evidence review for malaria in immigrant populations, see Appendix 9, available at www.cmaj.ca /lookup/suppl/doi:10.1503/cmaj.090313/-/DC1. 


\section{Depression}

Depression is a common and costly health care problem. ${ }^{383}$ Nearly all people with major depression are seen only in primary care, but up to $60 \%$ of cases go undetected and untreated. ${ }^{384}$ The level of underdiagnosis and inadequate treatment for depression is higher among migrants, who face cultural, linguistic and other barriers to accessing mental health care. ${ }^{385}$ Although migration in itself does not lead to an increase in depression, specific stressors and challenges can contribute to the onset of depression or influence its course, particularly among refugees. ${ }^{386}$ In general, immigrants to Canada have lower rates of depression than the general Canadian population, whereas refugees have comparable rates of depression but higher rates of post-traumatic stress disorder. ${ }^{387}$ Over time, the rate of depression in immigrant groups increases to match that of the general population. We undertook this review to determine whether existing approaches to screening for depression are appropriate for immigrants and refugees and to identify strategies that could improve the quality of care. The recommendations of the Canadian Collaboration for Immigrant and Refugee Health on screening for depression are outlined in Box 12A.

\section{Box 12A: Recommendations from the Canadian Collaboration for Immigrant and Refugee Health: depression}

If an integrated treatment program is available, screen adults for depression using a systematic clinical inquiry or validated patient health questionnaire (PHQ-9 or equivalent).

Link suspected cases of depression with an integrated treatment program and case management or mental health care.

\section{Basis of recommendations}

\section{Balance of benefits and harms}

The number needed to treat to prevent one case of persistent depression was 18 (95\% confidence interval 10-91) in studies of 1-12 months' duration. Treatment in enhanced depression-care models accounts for an additional $1 \%-2 \%$ reduction in depressive symptoms relative to usual care. The prevalence of depression is similar among Canadians and among immigrants and refugees $(10.7 \%)$, but access to care may be limited for migrants. No data on harms were reported, which would include patients' out-of-pocket costs and adverse effects of medication.

Quality of evidence Moderate

\section{Values and preferences}

The committee attributed more value to screening and treating depression to improve quality of life and less value to concerns about impairing rapport in therapeutic relationships, cultural acceptability and potential stigma of diagnostic labels, the cost and inconvenience of additional follow-up assessments, and the possible adverse effects or costs associated with treating patients with an incorrect diagnosis.

Note: PHQ-9 = nine-item Patient Health Questionnaire.

\section{Methods}

We used the 14-step approach developed by the Canadian Collaboration for Immigrant and Refugee Health ${ }^{16}$ (summarized in section 3 of this article, above). We considered the epidemiology of depression in immigrant populations and defined clinical preventive actions (interventions), outcomes and key clinical questions. We searched MEDLINE, Embase, CINAHL, PsychLIT, the Cochrane Library and other sources from Jan. 1, 1998, to Jan. 1, 2010. Detailed methods, search terms, case studies and clinical considerations can be found in the complete evidence review for depression (Appendix 10, available at www.cmaj.ca/lookup/suppl/doi:10.1503/cmaj $.090313 /-/ D C 1)$.

\section{Results}

Recommendations from the Canadian Task Force on Preventive Health Care $^{388}$ and the US Preventive Services Task Force $^{389,390}$ make scant mention of immigrants and refugees. In its guidelines for the treatment of depression, ${ }^{391}$ the American Psychiatric Association notes that language and other cultural variables may hamper accurate diagnostic assessment and treatment; it also mentions ethnic differences in the response to pharmacotherapy. The guidelines of the UK National Institute for Health and Clinical Evidence include statements on ethnic variations in prevalence and on the importance of social and cultural factors in choice of treatment. ${ }^{392}$ More recent studies, discussed in the complete evidence review (Appendix 10, available at www .cmaj.ca/lookup/suppl/doi:10.1503/cmaj.090313/-/DC1), provide evidence that can inform the implementation of screening for depression and integrated care for immigrants and refugees in primary care.

\section{What is the burden of depression in immigrant populations?}

The Canadian Community Health Survey (version 1.2) revealed a lifetime prevalence of depression of $10.8 \%$ in the general population. ${ }^{393}$ Immigrants who had arrived in Canada in the previous four years had the lowest rates of depression $(3.3 \%-3.5 \%)$. Among those who had arrived $10-14$ years ago (rate $8.5 \%$ ) or more than 20 years ago (rate $6.8 \%-7.2 \%$ ), rates were similar to those of the Canadian-born population. ${ }^{394}$ Proficiency in English or French and employment status did not affect these rates. A meta-analysis of studies on serious mental disorders among refugees found rates of depression similar to those in the general population but much higher levels of post-traumatic stress disorder, often in association with depression. ${ }^{51}$

Pregnancy and the postpartum period have been associated with symptoms of depression in immigrant women. ${ }^{395}$ Risk factors may include stressful life events, lack of social support 
or isolation, physical health problems, inability to speak the language of the host country, the demands of multiple roles and separation from children who have remained in the country of origin..$^{396,397}$

\section{Does screening for depression decrease morbidity and mortality?}

\section{Screening tools}

Many screening instruments for depression have been validated in primary care settings, and little evidence suggests that any particular instrument performs better than other instruments, although brief tools tend to be less specific. ${ }^{398}$ Both brief screening tools (two or three items) and longer ones tend to have relatively high false-positive rates $(60 \%-70 \%)$ when the prevalence of depression is $10 \%{ }^{399}$ Therefore, positive results on screening must be confirmed by a full diagnostic interview. Most screening instruments have not been validated for many of the immigrant groups commonly seen in primary care in Canada, although the patient health questionnaire has been validated with Chinese, South Asian and other populations.

\section{Relative benefits and harms of treatment}

In a systematic review of screening for depression conducted in 2002, the US Preventive Services Task Force found that clinical trials of integrated programs have demonstrated modest improvements in patient outcomes, but benefits have not been observed when screening results are simply reported to physicians without coordinated treatment and follow-up. ${ }^{400}$ Subsequent reviews have confirmed this finding..$^{401}$ Adverse effects among immigrants have not been systematically studied, but they may include impaired rapport and less use of general medical services if patients believe they are being labelled and stigmatized or are being treated improperly, the cost and inconvenience of additional follow-up assessments, and possible adverse effects or costs associated with treating patients with an incorrect diagnosis.
In a recent meta-analysis, Gilbody and associates ${ }^{398}$ found no benefit for screening alone, although there was some benefit in high-risk populations. However, a cumulative metaanalysis showed modest benefit when an integrated system of collaborative care was in place for follow-up (Table 12A). ${ }^{402}$ In a low-quality longitudinal study conducted in the United States, Wells and colleagues ${ }^{403}$ examined the effect of screening for depression within an integrated system of care, with follow-up by nurses and with other quality-associated improvements. The greatest improvement was seen for minority groups, specifically African Americans and Latinos. ${ }^{404}$

\section{Clinical considerations}

\section{Screening}

Screening should be conducted in a language in which the patient is fluent, either with translated instruments or through a trained interpreter. Cultural variations in presentation of symptoms, ways of coping and the stigma attached to mental health problems may complicate detection and treatment. ${ }^{405}$ The presence of prominent somatic symptoms and patients' tendency to attribute their depressed mood to somatic distress can also reduce primary care physicians' recognition of depression. ${ }^{406}$

Among refugee patients with depression, more than half also have post-traumatic stress disorder, and this comorbidity can complicate the recognition of depression..$^{51}$ Many cultures strongly stigmatize mental health problems, which may limit disclosure of behavioural or emotional difficulties. ${ }^{405}$ Depression can be distinguished from other forms of mental health problems and can be explained as a state of "energy depletion" and demoralization, which may provide a rationale for psychosocial assessment and treatment.

\section{Child-bearing women}

Guidelines from obstetrical groups have proposed that women be screened for depressive symptoms in each trimester of pregnancy, at 1-2 weeks postpartum, and possibly at 2,4 , and 6

Table 12A: Summary of findings for effects of collaborative care for depression

Patient or population: Patients with depression

Setting: Primary care

Intervention: Collaborative care

Comparison: Usual care

Source: Gilbody S, Bower P, Fletcher J, et al. Collaborative care for depression: a cumulative meta-analysis and review of longerterm outcomes. Arch Intern Med 2009;166:2314-21. ${ }^{402}$

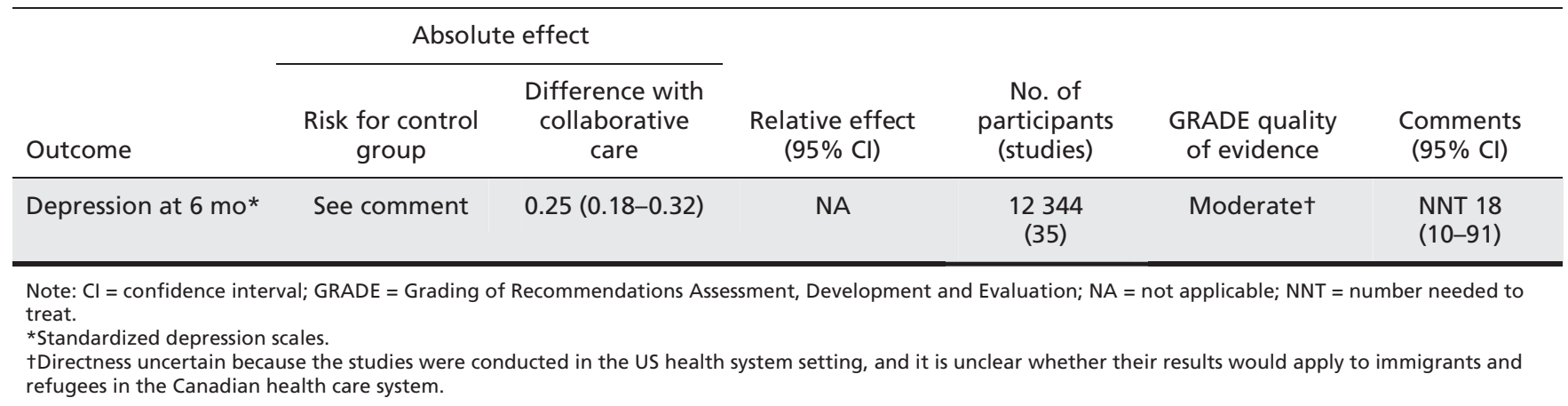


months postpartum. ${ }^{407-409}$ The Edinburgh Postnatal Depression Scale or nine-item patient health questionnaire have been used with immigrant women. Immigrant women's multiple roles in the home and the workplace may impede access to health services. ${ }^{395,396}$ Availability of child care facilities, transportation and support from family members and spouses can facilitate their seeking help. Group meetings can be an effective way to provide social support and health-promotion information.

\section{Adolescents and children}

The US Preventive Services Task Force has recommended screening adolescents (age 12-18 years) when integrated systems of treatment are available, including assessment, psychotherapy and follow-up. ${ }^{410,411}$ It is unclear which of the more than 30 available depression scales is best for screening and diagnosing depression among immigrant and refugee youth.

\section{Elderly people}

Migrant elderly people have not been well studied but may have a high risk of depression because of social isolation, loss of familiar surroundings and the changing nature of the family as members adapt to the new social context. ${ }^{412}$

\section{What are the potential implementation issues?}

Linguistic and cultural differences may constitute substantial barriers to recognition of depression and subsequent treatment negotiation and delivery. ${ }^{413,414}$ Medical interpreters, "culture brokers," bilingual and bicultural mental health practitioners, clinician training in cultural competence and cultural consultation may mitigate these potential barriers. ${ }^{52,415-417}$ Screening for depression produces benefits only when it is linked to an integrated system of care. An integrated system involves the following elements: systematic patient education, availability of allied health professionals to support continuity of care, frequent follow-up, a caseload registry to track patients, caseload supervision by a psychiatrist if indicated, stepped care and a plan for preventing relapse. ${ }^{418}$ Stepped care involves a progression of levels from patient education and self-management to medication or psychotherapy and, for complex cases, referral to a mental health practitioner. ${ }^{419}$

The clinical relationship is central to detection and treatment of mental health problems in primary care. Screening with structured questionnaires cannot replace clinical sensitivity, systematic inquiry and relationship-building. Given the great diversity of immigrant and refugee patients, no single approach is likely to be sufficient for optimal recognition and appropriate treatment of depression.

\section{Recommendations of other groups}

The Canadian Task Force on Preventive Health Care recommends screening adults for depression in primary care when integrated systems that include diagnostic, treatment and follow-up components are in place. ${ }^{388}$ The US Preventive Services Task Force recommends screening adolescents (age 12-18 years) when integrated systems of treatment, including assessment, psychotherapy and follow-up, are in place; however, it concludes that evidence is insufficient to make any recommendation for children $7-11$ years of age. ${ }^{410,411}$ Our recommendations highlight the value of screening for depression in the context of integrated treatment programs.

\section{Take-home messages}

- Rates of depression are lower among new immigrants to Canada, but over time these rates generally rise to match the rate in the general population.

- The prevalence of depression among refugees is comparable to that in the general population.

- Existing guidelines for depression suggest that all patients should be screened for depression when integrated systems are in place to provide follow-up treatment.

- For immigrants, use information about depression in relevant languages, translated screening questions and trained interpreters, as well as systematic inquiries about losses, stressors and symptoms.

- Moderate to severe depression should be treated with a stepped-care model, beginning with psychoeducation and antidepressant medication, close follow-up and culturally appropriate counselling.

For the complete evidence review for depression in immigrant populations, see Appendix 10, available at www.cmaj.ca/lookup /suppl/doi:10.1503/cmaj.090313/-/DC1.

More detailed information and resources for screening, assessment and treatment of depression can be found at: www.mmhrc.ca. 


\section{Post-traumatic stress disorder}

A large proportion of new immigrants to Canada come from countries experiencing social turmoil, and some are directly affected by protracted conflicts or war ${ }^{420}$ Refugees and others who face significant trauma and loss are at risk for mental health consequences, including post-traumatic stress disorder. For three main reasons, primary care practitioners play a key role in the recognition and management of post-traumatic stress disorder in immigrants and refugees. First, immigrants and refugees underutilize formal mental health services. ${ }^{420}$ Second, an integrated treatment approach is often needed for extreme traumas, common in refugees, such as torture and rape, which have severe and long-lasting consequences for both physical and mental health. ${ }^{421}$ Third, a family perspective is essential because trauma stemming from organized violence tends to affect the whole family, particularly children, who may not display dramatic or easily recognizable symptoms. We conducted an evidence review to determine the burden of post-traumatic stress disorder within immigrant and refugee populations, to evaluate the effectiveness of screening and treatment, and to identify barriers for primary care. The recommendations of the Canadian Collaboration for Immigrant and Refugee Health on post-traumatic stress disorder are outlined in Box 13A.

\section{Box 13A: Recommendations from the Canadian Collaboration for Immigrant and Refugee Health: post-traumatic stress disorder}

Do not conduct routine screening for exposure to traumatic events, because pushing for disclosure of traumatic events in well-functioning individuals may result in more harm than good.

Be alert for signs and symptoms of post-traumatic stress disorder, especially in the context of unexplained somatic symptoms, sleep disorders or mental health disorders such as depression or panic disorder, and perform clinical assessment as needed to address functional impairment.

\section{Basis of recommendation}

\section{Balance of benefits and harms}

Many persons who have been exposed to trauma do fine once they find safety and social supports. Brief screening instruments overestimate the rate of disease because they focus on symptoms and do not measure functional impairment. Detailed inquiry and pushing for disclosure without indications of distress or disorder could be harmful. There are no clinical trials demonstrating the benefits of routine screening for post-traumatic stress disorder.

\section{Quality of evidence}

Low (evidence available for refugee populations)

\section{Values and preferences}

The committee attributed more value to preventing potential harms from routine screening in the absence of clear evidence of benefits and determined that posttraumatic stress disorder was best dealt with through primary care practitioners remaining alert for signs and symptoms of this condition and performing clinical assessment to address functional impairment.

\section{Methods}

We used the 14-step method developed by the Canadian Collaboration for Immigrant and Refugee Health ${ }^{16}$ (summarized in section 3 of this article, above). We considered the epidemiology of post-traumatic stress disorder in immigrant populations and defined clinical preventive actions (interventions), outcomes and key clinical questions. We searched MEDLINE, Embase, CINAHL, PsychLIT, the Cochrane Library and other sources from Jan. 1, 2002, to Dec. 31, 2010. Detailed methods, search terms, case studies and clinical considerations can be found in the complete evidence review for post-traumatic stress disorder (Appendix 11, available at www.cmaj.ca/lookup/suppl/doi:10.1503/cmaj.090313/-/DC1).

\section{Results}

We identified 16 systematic reviews relevant to immigrants and refugees and five guidelines. We selected the 2005 guidelines commissioned by the National Institute for Clinical Excellence for the management of post-traumatic stress disorder in primary care, ${ }^{422}$ but none of the selected intervention studies in those guidelines provided evidence for immigrants or refugees. We also selected four Cochrane reviews on treatment of post-traumatic stress disorder, ${ }^{423-426}$ the practice guidelines from the International Society for Traumatic Stress Studies $^{427}$ and a systematic review on treatment of this condition in refugees and asylum seekers. ${ }^{428}$

\section{What is the burden of illness of post- traumatic stress disorder in immigrant populations?}

Most persons who experience traumatic events have a favourable mental health prognosis. ${ }^{53}$ When symptoms of post-traumatic stress disorder or acute stress disorder develop, there is, in most cases, substantial natural recovery (estimated at about $80 \%$ ). However, those in whom posttraumatic stress develops may remain symptomatic for years and are at risk of secondary problems, such as substance abuse..$^{54} \mathrm{~A}$ meta-analysis of studies involving adult refugees resettled in developed countries reported a 9\% prevalence of post-traumatic stress disorder, and 5\% had major depression. Among refugees with major depression, $71 \%$ also had posttraumatic stress disorder. Conversely, $44 \%$ of refugees with post-traumatic stress disorder also had major depression. ${ }^{51}$ Studies of child refugees report $11 \%$ prevalence of posttraumatic stress disorder. ${ }^{429,430}$ Symptoms may be reactivated when faced with new traumas, particularly if reminiscent of earlier traumatic experiences. ${ }^{431}$ Torture and cumulative trauma are the strongest predictors of post-traumatic stress disorder and are associated with chronic physical and mental health problems ${ }^{430}$ Fear of repatriation may exacerbate consequences of premigratory traumas. 
Longitudinal studies from Canada indicate that most adults and children with refugee status adapt well, in spite of a high level of exposure to premigratory trauma ${ }^{432,433}$ A populationbased health survey from Quebec similarly found that nonrefugee immigrants also experienced high levels of premigratory trauma, but that most immigrants were in good mental health. ${ }^{434}$

\section{Does screening for post-traumatic stress disorder decrease morbidity and mortality?}

\section{Screening}

Several short screening instruments practical for primary care settings have been developed. ${ }^{435}$ The four-item primary care post-traumatic stress disorder screening scale ${ }^{436}$ and the Breslau seven-item screening scale (available at http://ajp .psychiatryonline.org/cgi/content/full/156/6/908\#T2) are two simple means of identifying symptoms in primary care patients. In both cases, their cultural validity is unknown. Very few screening instruments have been tested for diagnostic accuracy among immigrants, refugees and asylum seekers. However, it may be reasonable to use questionnaires to assist in identifying symptoms, as part of a clinical assessment when addressing functional impairment.

\section{Relative benefits and harms of psychological treatment (adults and children)}

The systematic review and meta-analysis commissioned by the National Institute for Clinical Excellence ${ }^{422}$ provided evidence that psychological treatments, including trauma-focused cognitive-behavioural therapy and eye movement desensitization and processing, reduce the symptoms of post-traumatic stress disorder. We rated the quality of this evidence as low because of study limitations and inconsistency of results. Two Cochrane reviews ${ }^{425,426}$ provided similar evidence of effectiveness. A recent systematic review ${ }^{428}$ showed that psychological treatments (cognitive-behavioural therapy and narrative exposure therapy) can reduce symptoms of post-traumatic stress disorder among refugees, but we rated this evidence as very low quality. Other authors have reported that patients may experience adverse effects with therapy, such as re-experiencing traumatic events, and rates of withdrawal from active therapy may approach $30 \% .{ }^{437}$

\section{Clinical considerations}

\section{What are the potential implementation issues?}

Primary care practitioners need to be aware that immigrants and refugees may have been exposed to traumatic events. If a patient discloses a traumatic experience, it may be helpful to acknowledge the pain and suffering associated with the experience, to explain that a reaction is common for anyone who has undergone trauma and to offer empathetic reassurance that the situation is likely to get better. Several Canadian cities have centres and experts available to help care for survivors of trauma and torture.

Exploration of trauma and its consequences is not recom- mended in the first meeting with a patient unless it is the patient's primary complaint. However, certain symptom presentations should alert clinicians to the need for assessment for post-traumatic stress disorder, including unexplained physical complaints, sleep disorders, ${ }^{422}$ depression, panic disorder and somatoform disorder. ${ }^{51}$ Other presentations, such as severe dissociation mimicking brief reactive psychosis, dissociative disorders (amnesia and conversion) and psychotic depression, although less frequent, may also be related to post-traumatic stress disorder. Key elements of the assessment include level of psychological distress, the impairment associated with the symptoms in the patient and his or her family, substance abuse and suicidality.

Familiarity with the cultural background of the patient is recommended, and assessment should involve a professional interpreter if the patient's language ability is inadequate to express psychological distress and narrate the experience. ${ }^{422}$ Disclosing traumatic experience through relatives, family members or, particularly, children can be traumatic. ${ }^{438}$

Although not supported by clinical trials, the National Institute for Clinical Excellence ${ }^{422}$ recommends a phased intervention model, reflecting a pragmatic approach for refugees and asylum seekers who face the possibility of being returned to a traumatic environment. Phase I is defined as the period in which safety has not yet been established and during which intervention should focus on practical, family and social support. Phases II and III should focus on the patient's priorities, which may include social integration and/or treatment of symptoms. Unemployment, isolation and discrimination may overshadow the efficacy of mental health treatment in many patients ${ }^{430}$ which suggests that multifaceted interventions that include primary care, community organizations and other social institutions may be effective. ${ }^{432}$

\section{Recommendations of other groups}

The UK National Institute for Clinical Excellence ${ }^{422}$ recommends against routine systematic provision of brief, singlesession interventions. It recommends that consideration be given to the use of a brief screening instrument to detect posttraumatic stress disorder among refugees and asylum seekers, but does not suggest any specific instrument for screening or provide evidence of effectiveness of treatment in refugees. It also recommends that children and youth with post-traumatic stress disorder be offered a course of trauma-focused cognitive behaviour therapy. For sleep disorders, the National Institute for Clinical Excellence recommends the short-term use of hypnotic medication for adults or, if longer-term treatment is required, the use of suitable antidepressants to reduce the risk of dependence. For significant comorbid depression or severe hyperarousal, the National Institute for Clinical Excellence recommends paroxetine and mirtazapine. The US Centers for Disease Control and Prevention state that, in general, the majority of people who experience reactions to stress after disasters and emergencies show resilience and do not go on to experience long-term psychopathology ${ }^{439}$ Our recommendations highlight the paucity of evidence for routine screening and the potential for harms. 


\section{Take-home messages}

- Forty percent of Canadian immigrants and refugees from countries involved in war or with significant social unrest have been exposed to traumatic events before migration.

- Most (estimated at $80 \%$ ) individuals who experience traumatic events heal spontaneously after reaching safety.

- Empathy, reassurance and advocacy are key clinical elements of the recovery process.

- Pushing for disclosure of traumatic events by well-functioning individuals may result in more harm than good.
For the complete evidence review for post-traumatic stress disorder in immigrants, see Appendix 11, available at www .cmaj.ca/lookup/suppl/doi:10.1503/cmaj.090313/-/DC1.

More detailed information and resources for assessment and treatment of trauma and survivors of torture can be found at: www.mmhrc.ca. 


\section{Child maltreatment}

Child maltreatment is an important public health problem worldwide. ${ }^{400}$ The 2003 Canadian incidence study of reported child abuse and neglect estimated an incidence rate of 22 per thousand for child maltreatment. ${ }^{441}$ Of reported cases, $15 \%$ involved emotional maltreatment, $28 \%$ involved exposure to domestic violence, $24 \%$ involved physical abuse, $30 \%$ involved neglect and 3\% involved sexual abuse. Surveys conducted with nonrepresentative ethnic minority samples (which have likely included immigrants and refugees) have yielded higher rates of maltreatment than appear in official reports. ${ }^{42}$ This review was undertaken to clarify reports of child maltreatment in ethnic communities, to determine whether existing tools to screen for child maltreatment are appropriate for immigrant and refugee children, and to recommend strategies to improve the quality of care for these populations. The recommendations of the Canadian Collaboration for Immigrant and Refugee Health related to child maltreatment are outlined in Box 14A.

\section{Methods}

We used the 14-step approach developed by the Canadian Collaboration for Immigrant and Refugee Health ${ }^{16}$ (summarized in section 3 of this article, above). We considered the epidemiology of child maltreatment in immigrant populations and defined clinical preventive actions (interventions), outcomes and key clinical questions. We searched MEDLINE, Embase CINAHL, PsychLIT, the Cochrane Library and other sources from Jan. 1, 1995, to Dec. 31, 2010. Detailed methods, search terms, case studies and clinical considerations can be found in the complete evidence review for child maltreatment (Appendix 12, available at www.cmaj.ca/lookup/suppl/doi:10.1503 /cmaj.090313/-/DC1).

\section{Results}

We found no systematic reviews or guidelines on screening, prevention or treatment for child maltreatment in recently settled immigrants or refugees. The general literature search identified 180 titles with reference to child maltreatment. Seventeen citations were selected, and five key reviews retained as evidence. ${ }^{443-447}$ Studies conducted with general population and ethnic minority samples provided additional evidence that informed our recommendations related to child maltreatment among immigrants and refugees.

\section{What is the burden of child maltreatment in immigrant populations?}

The prevalence and incidence of child maltreatment among immigrant and/or refugee children in Canada are unknown. The evidence on maltreatment among ethnic minority children in the United States and Canada suggests that some ethnic minority children are disproportionately over- and under-represented in child protection services. ${ }^{448}$ These children are more likely to be screened for child maltreatment and also more likely to be reported to child protection services. Higher rates of screening result in a higher rate of

\section{Box 14A: Recommendations from the Canadian Collaboration for Immigrant and Refugee Health: child maltreatment}

\section{Screening}

Do not conduct routine screening for child maltreatment.

Be alert for signs and symptoms of child maltreatment during physical and mental examinations, and assess further when reasonable doubt exists or after patient disclosure.

\section{Basis of recommendations}

\section{Balance of benefits and harms}

The committee recommends against routine screening because of poor performance of screening instruments and the potential harms caused by the very high false-positive rates. Sensitivity ranged between $25 \%$ and $100 \%$, specificity between $16.5 \%$ and $94.3 \%$, and positive predictive value (when available) between $1.7 \%$ and $28.2 \%$.

\section{Quality of evidence}

Low

\section{Values and preferences}

The committee attributed more value to evidence for the negative effects of screening in relation to the high potential for harms. Harms could result from false positives leading to inappropriate labelling, psychological distress, inappropriate family separation, impaired clinician-patient rapport, potential reduction in use of general medical services and legal ramifications associated with involvement of child protection services.

\section{Prevention of child maltreatment and associated} outcomes

A home visitation program encompassing the first two years of life should be offered to immigrant and refugee mothers living in high-risk conditions, including teenage motherhood, single parent status, social isolation, low socioeconomic status, or living with mental health or drug abuse problems.

\section{Basis of recommendation}

\section{Balance of benefits and harms}

Home visitation programs for high-risk mothers, provided by nurses, reduced days in hospital for children $(p<0.001)$. Harms from surveillance and reporting to child protection services were not clearly demonstrated.

\section{Quality of evidence}

Moderate

\section{Values and preferences}

The committee attributed more value to supporting high-risk mothers with an offer of a home visitation program to provide practical support for families and the program's potential to improve health outcomes for children than to the potential risks associated with increased reporting to child protection services. 
inappropriate referral to child protection services. Ethnic minority children who received medical examinations were twice as likely $(p<0.001)$ to be reported to child protection services. ${ }^{55}$

The Canadian incidence study of reported child abuse and neglect ${ }^{441}$ found that ethnic minority children had a 1.8 times greater likelihood to be over-represented, whereas white and Arab children were under-represented. The higher rates were found among Aboriginals, Blacks, Latinos and Asians (the latter group for only physical abuse). This racial bias ${ }^{49}$ may be one explanation why ethnic minority children are disproportionately represented at all levels of the child protection process, ${ }^{450-452}$ despite the fact that they do not seem to be at higher risk of maltreatment. ${ }^{453}$ Another explanation may be professionals' divergent views as to what should be considered grounds for clinical suspicion of child maltreatment, ${ }^{454}$ which is associated with recency of training in child abuse, prejudices about the perpetrator ${ }^{454,455}$ and the professionals' beliefs in the positive or negative consequences of reporting a given family to child protection services. ${ }^{454}$

\section{Does screening for child maltreatment reduce harm and premature death or disability?}

\section{Screening tools}

Most screening methods consist of self-administered questionnaires generally completed by the mother, interviews or checklists completed by the professional who collects information directly from the child or clinical judgments by nurse or professional teams. ${ }^{443,445}$ All screening methods attempt to predict child maltreatment on the basis of either parents' potential for maltreatment or the presence or level of risk factors associated with maltreatment, rather than on the occurrence of actual maltreatment. Three systematic reviews have reported that these instruments tend to have high sensitivity but poor specificity and false-positive rates too high for use in clinical settings. ${ }^{443-445}$ Sensitivity ranged between $25 \%$ and $100 \%$, specificity between $16.5 \%$ and $94.3 \%$, and positive predictive value (when available) between $1.7 \%$ and $28.2 \%$.

Table 14A: Summary of findings for home visitation by nurses to prevent child maltreatment

Patient or population: Pregnant first-time mothers with at least one "sociodemographic risk characteristic" Settings: US clinic with free prenatal services and private obstetricians' offices; ${ }^{462}$ US public system of obstetric care ${ }^{459}$ Intervention: Home visitation by nurses

Comparison: Usual care

Sources: MacMillan HL; Canadian Task Force on Preventive Health Care. Preventive health care, 2000 update: prevention of child maltreatment. CMAJ 2000;163:1451-8. ${ }^{444}$ Olds DL, Eckenrode J, Henderson CR Jr, et al. Long-term effects of home visitation on maternal life course and child abuse and neglect: fifteen-year follow-up of a randomized trial. JAMA 1997;278:637-43. ${ }^{459}$ Kitzman H, Olds DL, Henderson CR Jr, et al. Effect of prenatal and infancy home visitation by nurses on pregnancy outcomes, childhood injuries, and repeated childbearing: a randomized controlled trial. JAMA 1997;278:644-52. ${ }^{462}$

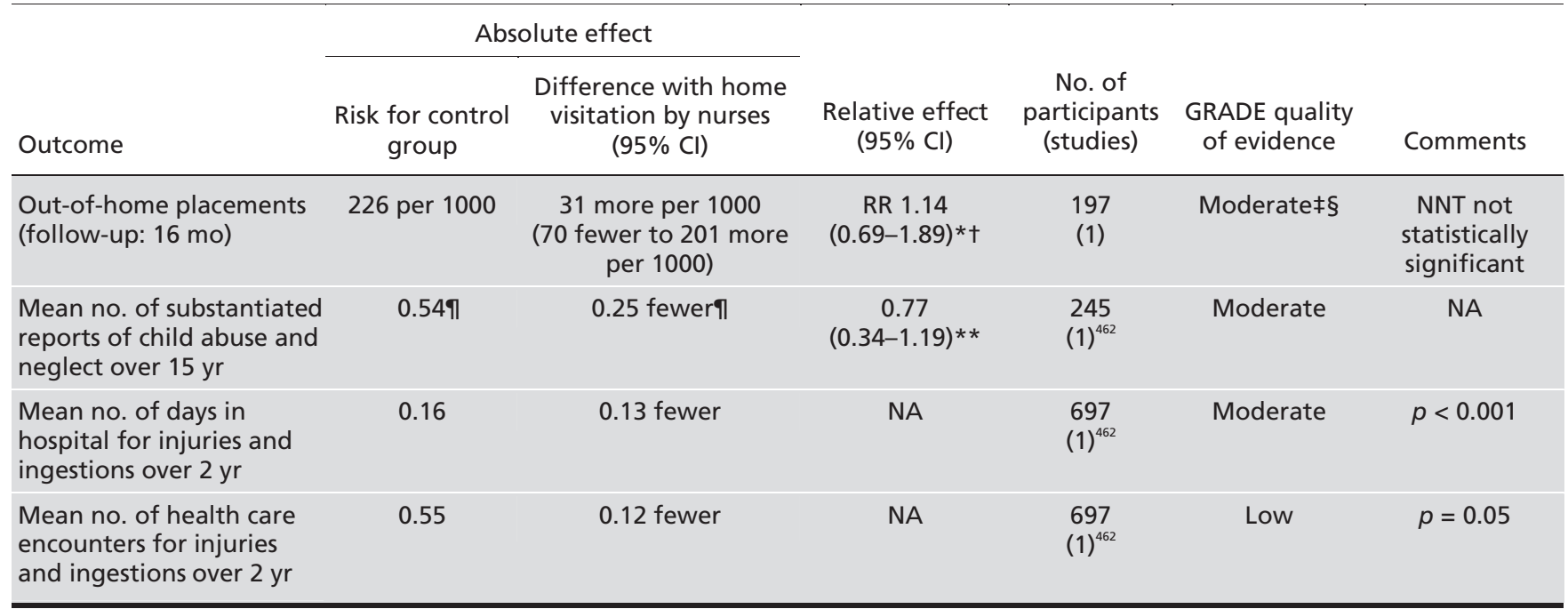

Note: $\mathrm{Cl}=$ confidence interval; GRADE = Grading of Recommendations Assessment, Development and Evaluation; NA = not applicable; NNT = number needed to treat; $\mathrm{RR}=$ risk ratio.

*Calculated using http://statpages.org/ctab2x2.html.

†Because RR crosses 0 (i.e., not statistically significant), the NNT could not be estimated.

‡Pregnant women with "specified psychosocial risk factors": substance abuse, homelessness, domestic violence, psychiatric illness, incarceration, HIV infection or lack of social support.

$\S$ "When the recommendation is in favour of an intervention and the $95 \%$ confidence interval (or alternative estimate of precision) around the pooled or best estimate of effect includes no effect and the upper confidence limit includes an effect that, if it were real, would represent a benefit that would outweigh the downsides" (GRADE Pro software).

TAdjusted for socioeconomic status, marital status, maternal age, education, locus of control, support from husband or boyfriend, working status, and husband or boyfriend use of public assistance at registration.

**Estimate $=$ (comparison log incidence) - (intervention log incidence). 


\section{Relative benefits and harms from screening}

False-positive ratings, which are the most common result in low-risk populations, can lead to a number of negative consequences, such as inappropriate labelling and punitive attitudes, psychological distress, ${ }^{56}$ inappropriate separation of children from family support systems, destruction of family supports, loss of resources and loss of autonomy for those falsely accused. ${ }^{456}$ This may leave parents wary of any subsequent assistance that may be offered, ${ }^{56}$ thus reducing their access to care. A systematic review of the performance of screening tests concluded that adding a screening protocol to the clinical encounter yielded additional false-positives that exceeded additional abused children detected. ${ }^{457}$

Compared with the general population, immigrant and refugee families may be more likely to suffer from the direct and indirect harms related to screening. Screening instruments have not been culturally validated and are less likely to be accurate because of factors such as language barriers, different cultural norms of behaviours and different attitudes toward institutional authority. ${ }^{458}$ Given the limited state of knowledge in immigrant populations, potential harms from routine screening for child maltreatment outweigh benefits, which have not yet been clearly established.

\section{Relative benefits and harms of preventing child maltreatment}

Home visitation programs by nurses aim to prevent child maltreatment by assessing and supporting families. To date, the 15 -year longitudinal study by Olds and associates ${ }^{459}$ has provided the best evidence for the effectiveness of a nursefamily partnership program in reducing actual child maltreatment. The effectiveness of this program is particularly evident for first-time mothers who are younger than 19 years of age, single or economically disadvantaged (Table 14A). ${ }^{44,446,460,461}$ Another prevention program (the Early Start Program) has also shown efficacy in reducing hospital admissions for child injuries at 36 months (17.5\% v. $26.3 \%$ for control group). ${ }^{463}$

\section{Relative benefits and harms of treatment for child maltreatment}

Several specific forms of intervention have been devised to reduce the consequences of child maltreatment. Traumafocused cognitive-behavioural therapy reduces sexually abused children's symptoms of anxiety, depression and sexual behaviour problems ${ }^{464}$ in both general population and ethnic minority children. Table 14B presents the outcomes of cognitive behavioural interventions. ${ }^{447}$ Parent-child interaction therapy ${ }^{465}$ showed a reduction in repeated reports of physical abuse in treatment relative to control groups (standard psychoeducational program) (19\% v. 49\%). In most other studies, the outcomes were not statistically significant but there was a consistent tendency in favour of treatment programs. The lack of evidence of efficacy for immigrant or

Table 14B: Summary of findings for cognitive-behavioural therapy for sexually abused children

Patient or population: Sexually abused children aged 2-18 yr

Settings: Unted States and Australia, communities and hospitals

Intervention: Cognitive-behavioural therapy for children

Comparison: Variable: group information-based approach, cognitive-behavioural therapy for parents and children, community control, wait-list control

Source: Macdonald G, Higgins JPT, Ramchandani P. Cognitive-behavioural interventions for children who have been sexually abused. Cochrane Database Syst Rev 2006;(4):CD001930. ${ }^{447}$

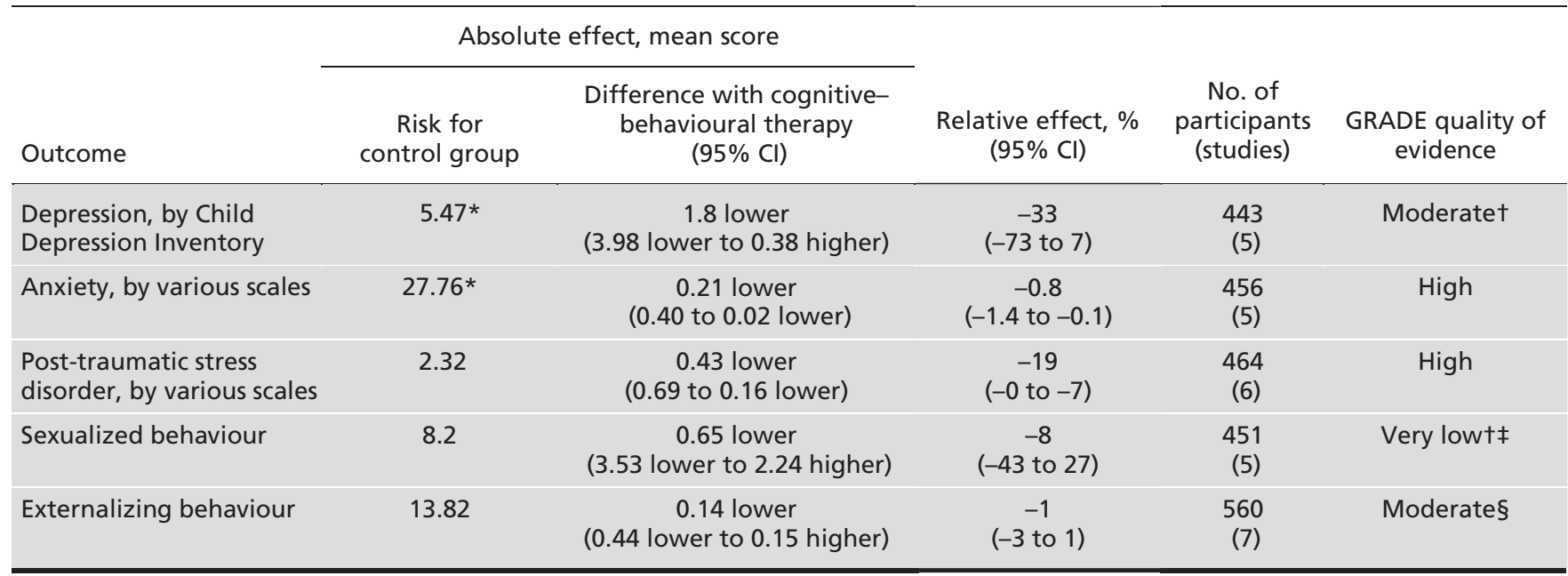

Note: $\mathrm{Cl}=$ confidence interval; GRADE = Grading of Recommendations Assessment, Development and Evaluation.

*Representative study chosen on basis of sample size.

$+95 \% \mathrm{Cl}$ includes no effect and the upper or lower confidence limit crosses the minimal important difference (MID), either for benefit or harm. (GRADE Pro software recommends that "if the MID is not known or the use of different outcomes measures required calculation of an effect size [ES], we suggest downgrading if the upper or lower confidence limit crosses an effect size of 0.5 in either direction.")

$\neq$ Test for heterogeneity $p=0.02$.

$\S$ Test for heterogeneity $p=0.01$. 
refugee children precludes extrapolation of the findings to these groups.

\section{Clinical considerations}

\section{What are the potential implementation issues?}

Some forms of child discipline may be unusual or outside Canadian social norms but are not pathological ${ }^{466}$ or dangerous for the child. Immigrant or refugee families may resort to other disciplinary behaviours (e.g., hitting a child with an object) that are condoned in their cultural context but that contravene child protection laws in Canada. Some cultural practices (e.g., scarification as part of life cycle rituals among some African children or cupping, a common traditional healing method in some Asian cultures that leaves circular ecchymoses) may be misinterpreted as signs of child abuse. Other culture-specific practices (e.g., female genital cutting) contravene child protection and civil laws in Canada. In situations where child maltreatment is suspected, observed or disclosed, the practitioner must take action in accordance with the child protection law in his or her region.

Language barriers, fear of separation from the child, fear of punitive institutional power and fear of deportation may constitute major barriers to disclosure of child maltreatment. Failure to investigate family dynamics and intergenerational conflicts, after disclosure of maltreatment by an immigrant child, may further disempower the parents and attribute greater power to the child, consequently aggravating his or her problem. Immigrant and refugee children placed in foster care may suffer from loss of connection with language of origin and religious, familial and cultural traditions. As a preventive strategy, clinicians may want to provide families with sources of information about their province's child protection law, their legal rights and their obligations regarding children, in addition to addressing other risk factors for child maltreatment. Recent research is showing that the SEEK (Safe Environment for Every Kid) model is promising. ${ }^{467}$

\section{Recommendations of other groups}

The US Preventive Services Task Force concluded that there is insufficient evidence for or against routine screening of child abuse. ${ }^{456}$ The Canadian Task Force on Preventive Health Care concluded that there is fair evidence to exclude screening for child maltreatment. ${ }^{79}$ The American Academy of Paediatrics ${ }^{468}$ and the American Medical Association ${ }^{469,40}$ do not support universal screening, but recommend that physicians be alert for signs and symptoms of child maltreatment during routine physical examination. The Task Force on Community Preventive Services of the US Centers for Disease Control and Prevention recommends early childhood home visitation for the prevention of child maltreatment in high-risk families and families with low-birth-weight infants. ${ }^{41}$ Our recommendations highlight the importance of prevention and the potential harms of routine screening in the context of cultural and linguistic diversity.

\section{Take-home messages}

- Children from ethnic minorities, including recently settled immigrants and refugees, are eight times more likely to be subjected to screening for child maltreatment than children in the general population.

- Immigrant and refugee families may be particularly vulnerable to the harms that can occur because of legal and institutional interventions consequent to false-positive screening results, such as over-reporting for child maltreatment and unnecessary separation of the child from his or her family.

For the complete evidence review for child maltreatment in immigrant populations, see Appendix 12, available at www .cmaj.ca/lookup/suppl/doi:10.1503/cmaj.090313/-/DC1.

More detailed information and resources on cultural aspects of child maltreatment can be found at: www.mmhrc.ca. 


\section{Intimate partner violence}

Intimate partner violence, defined as physical, emotional, financial and/or sexual abuse perpetrated against the victim by his or her intimate partner, ${ }^{472}$ is a significant public health problem worldwide. ${ }^{40}$ In Canada, a 1999 study of a nationally representative sample of 26000 participants reported $8 \%$ intimate partner violence against a female and $7 \%$ against a male by a previous or current partner in the past five years. ${ }^{473}$ Women, however, are more likely than men to be the victims of serious violent acts such as sexual abuse, beatings $(25 \% \mathrm{v}$. $10 \%)$, being choked ( $20 \%$ v. $4 \%$ ) or being threatened or having a weapon used against them $(13 \%$ v. $7 \%){ }^{474}$ They are also more likely than men to be injured during the violent act $(40 \%$ v. $13 \%)$ and to be fearful for their lives $(40 \%$ v. $<10 \%) .{ }^{474} \mathrm{In}$ this review we aimed to determine whether existing screening tools and approaches for intimate partner violence are appropriate for immigrant and refugee women and to identify care barriers for these populations. The recommendations of the Canadian Collaboration for Immigrant and Refugee Health related to intimate partner violence are outlined in Box 15A.

\section{Methods}

We used the 14-step approach developed by the Canadian Collaboration for Immigrant and Refugee Health ${ }^{16}$ (summarized in section 3 of this article, above). We considered the epidemiology of intimate partner violence in immigrant populations and defined clinical preventive actions (interventions), outcomes and key clinical questions. We searched MEDLINE, Embase, CINAHL, PsychLIT, the Cochrane Library and other sources from Jan. 1, 1995, to Dec. 31, 2010. Detailed methods, search terms, case studies and clinical considerations can be found in the complete evidence review for intimate partner violence (Appendix 13, available at www .cmaj.ca/lookup/suppl/doi:10.1503/cmaj.090313/-/DC1).

\section{Results}

We found no systematic reviews or evidence-based guidelines on screening, prevention or treatment for intimate partner violence in immigrants or refugees. The general literature search identified 409 titles on intimate partner violence, and after appraisals, we retained two key reviews as evidence. ${ }^{475,476}$ After the search update, we selected two additional key reviews and one randomized controlled trial. ${ }^{477-479}$ Studies conducted with general population and ethnic minority samples informed our clinical recommendations.

\section{What is the burden of intimate partner violence in immigrant populations?}

Three studies provided secondary analyses of the 1999 Statistics Canada General Social Survey. Women born in developing countries reported the highest prevalence rates of intimate partner violence, followed by Canadian-born women and immigrant women from developed countries. However, when all other variables in the model were controlled for, the analysis showed that recently settled immigrant women (i.e., in Canada for less than 10 years) had significantly lower odds of intimate partner violence victimization than longer-term immigrants and Canadian-born women. ${ }^{480}$ Single, divorced, separated or widowed immigrant women were 10 times more likely to report intimate partner violence than immigrant women married or in a common-law relationship. ${ }^{481}$ Immigrant women reported higher rates of emotional abuse than Canadian-born women $(14.7 \%$ v. $8.7 \%)$, with the strongest risk factor being their partner's low educational level. ${ }^{482}$

Regional surveys on intimate partner violence have yielded higher rates. MacMillan and colleagues ${ }^{483}$ reported rates that ranged from $4.1 \%$ to $17.7 \%$ for Canadian-born women and $12.6 \%$ for foreign-born women. Ahmad and coauthors ${ }^{484}$ reported a $22 \%$ rate of intimate partner violence following computer screening. Prevalence rates also vary in relation to the health care setting (highest prevalence in emergency departments). Finally, women in war zones, disaster zones, during flight or displaced in refugee camps in countries of asylum may be at higher risk for intimate partner violence. ${ }^{485}$

\section{Does screening for intimate partner violence reduce morbidity or mortality?}

\section{Screening tools}

Screening for intimate partner violence differs from tradi-

Box 15A: Recommendations from the Canadian Collaboration for Immigrant and Refugee Health: intimate partner violence

Do not conduct routine screening for intimate partner violence.

Be alert for potential signs and symptoms related to intimate partner violence, and assess further when reasonable doubt exists or after patient disclosure.

Basis of recommendation

\section{Balance of benefits and harms}

Current evidence does not demonstrate clear benefits from screening women for intimate partner violence, and harms have resulted from screening. Compared with the general population, there may be greater risk among immigrant and refugee women for harm directly related to screening (e.g., risk of loss of migration status and sponsorship agreements). Harm may occur indirectly through impaired patient-physician rapport and subsequent reduction in use of medical and mental health services.

\section{Quality of evidence}

Moderate

\section{Values and preferences}

The committee attributed more value to evidence of harms and lack of evidence of benefits and less value to recommending uncertain interventions, even in the face of significant concerns. 
tional screening for medical disorders because the target of clinical concern is a behavioural event, which women usually recognize as a problem but which they may not view as appropriate for medical attention. ${ }^{485,486}$ Four short self-report questionnaires have received the most study. The "Hurt, Insulted, Threatened, or Screamed at" questionnaire (four items) yields sensitivity ranging from $30 \%$ to $100 \%$ and specificity from $86 \%$ to $99 \% .{ }^{476}$ The Partner Violence Screen (three items) provides sensitivity from $35 \%$ to $71 \%$ and specificity from $80 \%$ to $94 \%{ }^{477}$ The Women Abuse Screening Tool (eight items) yields $47 \%$ sensitivity and $96 \%$ specificity. ${ }^{479}$ The Abuse Assessment Screen (five items) yields sensitivity ranging from $32 \%$ to $94 \%$ and specificity from $55 \%$ to $99 \% .487$

A Canadian randomized controlled trial found women preferred self-completed approaches. ${ }^{483}$ However, other studies comparing administration methods of screening instruments (e.g., face-to-face interviews, computer screening, written screening) have shown inconsistent results. ${ }^{48,488,489}$ Furthermore, it is unknown whether these results apply to immigrant and refugee women.

\section{Relative benefits and harms of screening}

A Canadian trial on the effect of screening found no statistically significant differences between women screened or not screened at 6,12 or 18 months follow-up for recurrence of intimate partner violence (Table 15A) ${ }^{478}$ More than half of the women who disclosed being victims of intimate partner violence on screening did not discuss the violence with their practitioner during the health care visit. An important study limitation was that no specific intervention was provided to women who disclosed or screened positive. ${ }^{478}$

Other studies have found screening benefits such as decreasing isolation, increasing support, relief, breaking the silence and validating women's feelings. ${ }^{485,490}$ However, these same studies identified several harms, including feeling that the practitioner is too busy or not interested, feeling judged and being disappointed by the practitioner's response, increased anxiety, concerns about privacy, ${ }^{484}$ breaches of confidentiality and legal repercussions, fear of being reported to child protective services, ${ }^{485}$ and concern about or actual increased risk of retaliation or further harm from the partner. ${ }^{485}$

\section{Relative benefits and harms of treatment}

The strongest evidence for treatment has come from studies of the Experimental Social Innovation and Dissemination program, ${ }^{491,492}$ which reported decreased physical and emotional abuse at 12-24 months follow-up and improvement of women's quality of life at 12 months follow-up. Ramsay and coworkers ${ }^{472}$ reported that, while promising, the results were inconclusive. In Table 15B, we report the efficacy of the Experimental Social Innovation and Dissemination advocacy and counselling intervention program in decreasing the incidence of intimate partner violence ${ }^{475}$ in an ethnically

Table 15A: Summary of findings on screening for intimate partner violence to reduce morbidity due to such violence

Patient or population: English-speaking female patients

Settings: Health care settings in Ontario

Intervention: Screening for intimate partner violence

Comparison: No screening

Source: Macmillan $\mathrm{HL}$, Wathen $\mathrm{CN}$, Jamieson $\mathrm{E}$, et al. Screening for intimate partner violence in health care settings: a randomized trial. JAMA 2009;302:493-501. ${ }^{478}$

\begin{tabular}{|c|c|c|c|c|c|c|}
\hline \multirow[b]{2}{*}{$\begin{array}{l}\text { Outcome } \\
\text { (18-mo follow-up) }\end{array}$} & \multicolumn{2}{|c|}{ Absolute effect } & \multirow[b]{2}{*}{$\begin{array}{l}\text { Relative effect } \\
\quad(95 \% \mathrm{Cl})\end{array}$} & \multirow[b]{2}{*}{$\begin{array}{l}\text { No. of } \\
\text { participants } \\
\text { (studies) }\end{array}$} & \multirow[b]{2}{*}{$\begin{array}{l}\text { GRADE } \\
\text { quality of } \\
\text { evidence }\end{array}$} & \multirow[b]{2}{*}{$\begin{array}{l}\text { Comments } \\
(95 \% \mathrm{Cl})\end{array}$} \\
\hline & $\begin{array}{l}\text { Risk for } \\
\text { control } \\
\text { group }\end{array}$ & $\begin{array}{l}\text { Difference with } \\
\text { screening } \\
(95 \% \mathrm{Cl})\end{array}$ & & & & \\
\hline $\begin{array}{l}\text { Intimate partner } \\
\text { violence, by } \\
\text { Composite Abuse } \\
\text { Scale }\end{array}$ & 530 per 1000 & $\begin{array}{c}74 \text { fewer per } 1000 \\
\text { (159 fewer to } 32 \text { more } \\
\text { per 1000) }\end{array}$ & $\begin{array}{c}\text { RR } 0.86 \\
(0.70-1.06)^{*}\end{array}$ & $\begin{array}{l}379 \\
(1)\end{array}$ & Moderate†‡ & $\begin{array}{l}\text { NNT not } \\
\text { statistically } \\
\text { significant }\end{array}$ \\
\hline $\begin{array}{l}\text { Post-traumatic stress } \\
\text { disorder screening, by } \\
\text { SPAN (startle, } \\
\text { physically upset by } \\
\text { reminders, anger, } \\
\text { numbness) }\end{array}$ & 601 per 1000 & $\begin{array}{l}162 \text { fewer per } 1000 \\
(246 \text { to } 66 \text { fewer } \\
\text { per } 1000)\end{array}$ & $\begin{array}{c}\text { RR } 0.73 \\
(0.59-0.89)^{*}\end{array}$ & $\begin{array}{r}379 \\
(1)\end{array}$ & Moderate†‡ & $\begin{array}{l}\text { NNT } 7 \\
(5-16)\end{array}$ \\
\hline $\begin{array}{l}\text { Quality of life, by } \\
\text { WHO Brief }\end{array}$ & $\begin{array}{c}\text { Mean score } \\
52.7\end{array}$ & $\begin{array}{l}\text { Mean score } 5.8 \text { higher } \\
\text { (2.14 to } 9.46 \text { higher) }\end{array}$ & NA & $\begin{array}{r}379 \\
(1)\end{array}$ & Moderate†§ & NA \\
\hline Depression & $\begin{array}{c}\text { Mean score } \\
24.4\end{array}$ & $\begin{array}{c}\text { Mean score } 3.4 \text { lower } \\
\text { (5.8 to } 1.0 \text { lower) }\end{array}$ & NA & $\begin{array}{r}379 \\
(1)\end{array}$ & Moderate†§ & NA \\
\hline
\end{tabular}

Note: $\mathrm{Cl}=$ confidence interval; GRADE = Grading of Recommendations Assessment, Development and Evaluation; NA = not applicable; NNT = number needed to treat; RR = relative risk; WHO = World Health Organization.

*Calculated using Review Manager on the basis of observed counts.

tOnly one study.

¥Dichotomous outcome: total number of events was less than 300 .

§Continuous outcome: total population size was less than 400 . 
diverse sample of women who had spent at least one night in a shelter.

\section{Clinical considerations}

\section{What are potential implementation issues?}

Signs and symptoms of intimate partner violence differ significantly among women. They may be absent in some women or be of a psychological (depression, anxiety, suicidal ideation, alcohol or drug abuse), social (social isolation) and/or physical (injuries, bruises and aches) nature in other women. Patient-physician rapport thus remains a key element in the detection of intimate partner violence.

Recently settled immigrant women in Canada are more likely to report intimate partner violence to the police than women in the general population but are less likely to use social services. ${ }^{494}$ Barriers to help-seeking included fear of deportation or not accessing Canadian citizenship, lack of knowledge of services or language-specific services, experiences of racism or discrimination. ${ }^{494}$ Culturally specific perceptions of spousal relationships, gender roles, negative experiences with authorities, aggression and abuse may affect reporting and disclosure. ${ }^{45}$ Involvement with police or criminal proceedings may put immigrant women at risk of losing their sponsorship agreements. ${ }^{485,494}$

Intimate partner violence is now considered a form of child maltreatment. Women may delay disclosure of violence because of fear of losing custody of their children (child protection services often cite the mother's failure to protect her children) ${ }^{485,494}$ In addition, some women feel coerced into staying in a shelter to keep custody of their children. Although this may protect them from further intimate partner violence, it may also isolate them from extended family and community networks that might otherwise be integrated effectively into the intervention plan. ${ }^{458}$

Services that can defuse conflict situations and reduce family stress include social welfare, reliable childcare, safe housing, language classes, and other educational and vocational training opportunities. Community grassroots organizations can provide information and support groups in appropriate languages and in a culturally competent manner. ${ }^{495-498}$ Research is beginning to show benefits when screening and interventions target women with specific conditions, for example pregnancy, mental illness and substance abuse, but this work has yet to consider the immigrant context.

\section{Recommendations of other groups}

National clinical preventive screening committees, the Canadian Task Force on Preventive Health Care, the UK National Screening Committee and the US Preventive Services Task Force have not found sufficient evidence to recommend for or against screening all women for intimate partner violence. ${ }^{476-478}$ The UK National Screening Committee concluded that "screening for domestic violence should not be introduced" in periodic health examinations. The American Medical Association, the American Academy of Family Physicians and the American College of Obstetricians and Gynecologists have recommended routinely screening all women for intimate partner violence. ${ }^{479} \mathrm{How}$ ever, these organizations have not based their recommendations on systematic reviews of effectiveness. Our guidelines

Table 15B: Summary of findings for advocacy programs to prevent further intimate partner violence

Patient or population: Women in a Midwest shelter program for women with abusive partners who had (i) spent at least one night in the shelter and (ii) planned on staying in the general vicinity for the first three months after leaving the shelter

Setting: Community setting

Intervention: Advocacy programs

Comparison: No advocacy program

Sources: Wathen CN, Macmillan HL. Interventions for violence against women: scientific review. JAMA 2003;289:589-600. ${ }^{475}$ Sullivan CM, Bybee DI. Reducing violence using community-based advocacy for women with abusive partners. J Consult Clin Psychol $1999 ; 67: 43-53 .{ }^{491}$

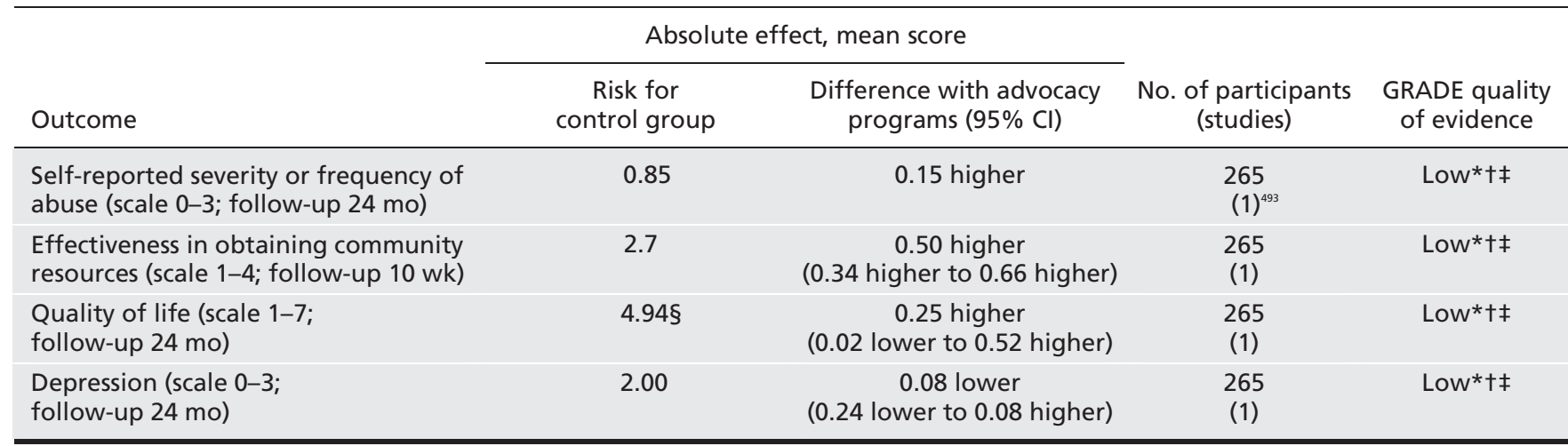

Note: $\mathrm{Cl}=$ confidence interval; GRADE = Grading of Recommendations Assessment, Development and Evaluation.

*Only one study.

tConcerns about directness and applicability only to women seen in primary care who have been in a shelter.

¥Fewer than 300 events.

$\S$ Postintervention scores. 
highlight the paucity of data on the effectiveness of screening programs and the concern for potential harms from routine screening.

\section{Take-home messages}

- The rate of reporting of intimate partner violence is lower among recently settled immigrant women than among longer-term immigrants and Canadian-born women.

- Linguistic barriers, financial dependencies, fear of losing custody of children and limited knowledge of laws and health services constitute significant barriers to both disclosure and adherence to interventions among immigrant and refugee women.
- To decrease the rate of abuse, practitioners should refer women who report spending at least one night in a shelter to a structured program of patient-centred (advocacy) support services.

For the complete evidence review for intimate partner violence in immigrant populations, see Appendix 13, available at www .cmaj.ca/lookup/suppl/doi:10.1503/cmaj.090313/-/DC1.

More detailed information and resources on cultural aspects of intimate partner violence can be found at: www.mmhrc.ca. 


\section{Type 2 diabetes mellitus}

More than two million Canadians have diabetes mellitus and, by the end of this decade, this number is expected to rise to three million. ${ }^{499}$ People of South Asian, Latin American and African ethnicity have a two to four times greater risk for development of type 2 diabetes than the white population. ${ }^{57,500}$ Up to 50\% of Canada's recent immigrants come from South Asia, Latin America and the Caribbean. ${ }^{57}$ The efficiency of diabetes screening improves when targeted to undiagnosed and high-risk populations. ${ }^{501} \mathrm{We}$ conducted an evidence review to estimate the diabetes burden for immigrant populations, to evaluate the effectiveness of screening for prevention and treatment, and to identify barriers and facilitators to prevention and treatment of diabetes in primary care. The recommendations of the Canadian Collaboration for Immigrant and Refugee Health on screening and treating prediabetes and diabetes are outlined in Box 16A.

\section{Methods}

We used the 14-step approach developed by the Canadian Collaboration for Immigrant and Refugee Health ${ }^{16}$ (summarized in section 3 of this article, above). We considered the epidemiology of diabetes in immigrant populations and defined clinical preventive actions (interventions), outcomes and key clinical questions. We searched MEDLINE, Embase, CINAHL, the Cochrane Library and other sources from Jan. 1, 1995, to July 14, 2010. Detailed methods, search terms, case studies and clinical considerations can be found in the complete evidence review for type 2 diabetes (Appendix 14, available at www .cmaj.ca/lookup/suppl/doi:10.1503/cmaj.090313/-/DC1).

\section{Results}

We found no randomized controlled trials evaluating the effect of diabetes screening programs on morbidity and mortality. We identified three reviews focusing on the genetic risk factors predisposing high-risk ethnic groups to diabetes. ${ }^{502-504}$ Additionally, we identified a systematic review ${ }^{505}$ and a metaanalysis $^{506}$ on the disparities in type 2 diabetes treatment between high-risk ethnic groups and the general white population and two systematic reviews on culturally appropriate treatment of type 2 diabetes in high-risk populations. ${ }^{507,508} \mathrm{An}$ updating search, building on the 2008 guidelines of the Canadian Diabetes Association ${ }^{509}$ and the systematic review of the US Preventive Services Task Force ${ }^{501}$ on type 2 diabetes, yielded an additional 21 articles, of which two were selected..$^{58,510} \mathrm{We}$ also identified a meta-analysis of randomized controlled trials ${ }^{511}$ that studied the impact of tight gylycemic controls on morbidity and mortality.

\section{What is the burden of type 2 diabetes in immigrant populations?}

The prevalence of diabetes is increasing worldwide, most notably in South Asia, sub-Saharan Africa and Latin America, regions that are major contributors to international migration. Contributory factors cited for increased prevalence of type 2 diabetes include genetic susceptibility, ${ }^{50,504}$ increasing sedentary lifestyle, changing food habits ${ }^{512}$ and increasing levels of adult and childhood obesity.

In Canada, up to $50 \%$ of new immigrants face a twofold to fourfold higher prevalence of type 2 diabetes than whites ${ }^{513}$ and experience a younger age of onset. ${ }^{514}$ A prevalence study in Ontario $^{57}$ reported higher prevalences for South Asians (odds ratio [OR] 4.01 for men, 3.22 for women), Latin Americans (OR 2.18 for men, 2.40 for women) and sub-Saharan Africans (OR 2.31 for men, 1.83 for women). The average age at diagnosis of diabetes is decreasing, for example, from 52.0 to 46.0 years in the United States $(p<0.05)^{514}$ and, most dramatically, in black, Asian and Hispanic populations. In India, for example, there is a significantly higher prevalence of type 2 diabetes in people 35 to 44 years $(8.5 \%-11.5 \%, p=0.05) .{ }^{515} \mathrm{Cen}$ tral adiposity (overweight or obesity) results in a higher degree of insulin resistance and hence confers a higher risk for diabetes and premature coronary artery disease. ${ }^{516} \mathrm{~A}$ study conducted in a high-risk population from Jamaica found that the sensitivity of waist circumference in predicting type 2 diabetes was $71 \%$ in men and $65 \%$ in women and that the specificity of this measure was $79 \%$ in men and $60 \%$ in women, ${ }^{517}$ which

\section{Box 16A: Recommendations from the Canadian Collaboration for Immigrant and Refugee Health: type 2 diabetes mellitus}

Screen immigrants and refugees $>35$ years of age from ethnic groups at high risk for type 2 diabetes (South Asian, Latin American and African) with fasting blood glucose.

\section{Basis of recommendation}

\section{Balance of benefits and harms}

Detecting impaired fasting blood glucose and treating with diet and exercise can delay the onset of diabetes (number needed to treat [NNT] 5, 95\% confidence interval [CI] 4-6). Treating patients with diabetes with intensive blood pressure interventions can decrease mortality (NNT $38,95 \% \mathrm{Cl} 23-203)$, and tight glucose control can decrease myocardial infarctions (NNT 131, Cl 87-298). People of South Asian, Latin American or African ethnicity face a twofold to fourfold higher prevalence of type 2 diabetes with earlier onset compared with white people. Minimal harms are reported for lifestyle interventions and adverse effects of antihyperglycemic agents.

\section{Quality of evidence}

Moderate

\section{Values and preferences}

The committee attributed more value to delaying the onset of diabetes than to the current uncertainty of impact on mortality for lifestyle interventions. The committee also attributed greater value to the potential to decrease morbidity and mortality with treatment of hypertension and hyperglycemia in high-risk ethnic populations than to concern about harms due to treatments. 
suggests that waist circumference can be a useful alternative to body mass index. The International Diabetes Federation has recommended the use of ethnospecific waist circumference as a cut-off parameter for screening these high-risk populations (Table 16A). ${ }^{518,519}$

\section{Does screening decrease the incidence of diabetes and/or related cardiovascular complications?}

\section{Screening tests}

Fasting blood glucose is considered the most appropriate screening test for diabetes because of its feasibility and specificity. ${ }^{510}$ A fasting blood glucose level of $7.0 \mathrm{mmol} / \mathrm{L}$ has a moderate sensitivity $(40 \%-87 \%)$, but good specificity $(96 \%-$ $99 \%$ ), to predict a blood glucose level of $11.1 \mathrm{mmol} / \mathrm{L}$ in a two-hour oral glucose tolerance test. A recent systematic review also concluded that hemoglobin $\mathrm{A}_{1 \mathrm{C}}$ and fasting blood glucose are equally effective screening tools, but hemoglobin $\mathrm{A}_{1 \mathrm{C}}$ is more costly than fasting blood glucose. ${ }^{520}$

\section{Relative benefits and harms of treatment}

The 2008 meta-analysis of the US Preventive Services Task Force on lifestyle interventions for prediabetes ${ }^{501}$ showed a significant decrease in onset of diabetes with minimum harms. We rated the quality of this evidence as high but noted the need to study the long-term effects of delaying diabetes onset (Table 16B). ${ }^{521}$ Additionally, we identified a Cochrane systematic review ${ }^{58}$ showing that culturally appropriate health education was effective in reducing blood glucose and increasing diabetes knowledge in ethnic minority groups. None of these trials reported significant adverse effects from lifestyle interventions. The evidence was insufficient to comment on the negative theoretical impact of labelling.

Blood pressure control in diabetes reduces cardiovascular morbidity and mortality. ${ }^{510,521}$ Trials reported minor adverse drug reactions such as cough with some antihypertensives and no harms related to the socioeconomic implications of diagnosis and treatment (Table 16C). ${ }^{522} \mathrm{~A}$ recent meta-analysis ${ }^{511}$ of five prospective randomized controlled trials evaluating the effect of intensive glucose control showed a small but significant decrease in myocardial infarctions in the treatment group, which had a hemoglobin $\mathrm{A}_{1 \mathrm{C}}$ level $0.9 \%$ lower than the control group, but no effect on events of stroke or mortality. Very intensive glucose control (hemoglobin $\mathrm{A}_{1 \mathrm{C}}<6.5 \%$ ) has not been shown to decrease mortality and has been associated with hypoglycemia. Populations and individuals who would benefit the most from screening have undiagnosed, longstanding disease, and intensive lifestyle and/or hypertension interventions would have the greatest absolute effect.

\section{Clinical considerations}

\section{What are the potential implementation issues?}

A Cochrane review ${ }^{523}$ found that diet and lifestyle interventions reduced weight and the incidence of diabetes, but these measures were more effective in younger populations. Others have found that diabetes education is less effective in situations of low socioeconomic status and cultural factors. Language barriers, migration stress and cultural diversity (diets, lifestyles, perceptions of weight, fasting at Ramadan) may complicate diabetes care and education.

Ethnic groups prefer culturally appropriate dietary and exercise advice. ${ }^{58}$ A Cochrane review ${ }^{58}$ on culturally appropriate health education showed improved glycemic control and diabetic knowledge scores compared with standard diabetic education approaches at 12 months. Other outcomes, such as lipid levels and blood pressure, showed no significant difference from control groups. Another review, ${ }^{524}$ looking at diabetic care in socially disadvantaged populations, identified interventions that may improve outcomes, including culturally tailored care, a focus on individuals in assessment and feedback, and incorporation of treatment algorithms.

\section{Recommendations of other groups}

The US Preventive Services Task Force recommends screening in all asymptomatic hypertensive populations ${ }^{525}$ and suggests population-targeted screening for those with hyperten-

Table 16A: Country- and ethnic-specific values for waist circumference ${ }^{518,519}$

\begin{tabular}{l}
$\begin{array}{l}\text { Country or ethnic } \\
\text { group }\end{array}$ \\
(as meast circumference, $\mathrm{cm}^{*}$ \\
\hline
\end{tabular}

\begin{tabular}{|c|c|}
\hline \multicolumn{2}{|l|}{ Europidt } \\
\hline Male & $\geq 94$ \\
\hline Female & $\geq 80$ \\
\hline \multicolumn{2}{|c|}{ South Asian $¥$} \\
\hline Male & $\geq 90$ \\
\hline Female & $\geq 80$ \\
\hline \multicolumn{2}{|l|}{ Chinese } \\
\hline Male & $\geq 90$ \\
\hline Female & $\geq 80$ \\
\hline \multicolumn{2}{|l|}{ Japanese§ } \\
\hline Male & $\geq 85$ \\
\hline Female & $\geq 90$ \\
\hline
\end{tabular}

Ethnic South and Central American

Use South Asian recommendations until more specific data are available

Sub-Saharan African

Eastern

Mediterranean and Middle East

Use European data until more specific data are available

Use European data until more specific data are available for (Arab) populations

*These are pragmatic cut points, and better data are required to link them to risk. Ethnicity rather than country of residence should be the basis for classification. In future epidemiologic studies of populations of Europid origin, preference should be given to using both European and North American cut points to allow better comparisons.

tIn the United States, the Adult Treatment Panel III values $(102 \mathrm{~cm}$ for males, $88 \mathrm{~cm}$ for females) are likely to continue to be used for clinical purposes.

\#Based on a Chinese, Malay and Asian-Indian population.

$\S$ Subsequent analyses suggested that Asian values $(90 \mathrm{~cm}$ for males, $80 \mathrm{~cm}$ for females) should be used for the Japanese population until more data are available. 
sion, advanced age and obesity. The Canadian Diabetes Association $^{509}$ recommends diabetes screening for all adults over 40 years of age. Earlier testing should be considered in people with additional risk factors, and ethnic-specific waist circumference cut-off points should be used for risk stratification. The American Diabetes Association ${ }^{526}$ recommends screening

Table 16B: Summary of findings for lifestyle modifications to prevent type 2 diabetes mellitus

Patient or population: Patients at high risk for type 2 diabetes, with impaired fasting glucose

Settings: Outpatient or community health clinics

Intervention: Lifestyle modifications for patients with impaired fasting glucose

Comparison: No intervention

Source: Harris R, Donahue K, Rathore SS, et al. Screening adults for type 2 diabetes: a review of the evidence for the U.S.

Preventive Services Task Force. Ann Intern Med 2003;138:215-29. ${ }^{521}$

\begin{tabular}{|c|c|c|c|c|c|c|}
\hline Outcome & \multicolumn{2}{|c|}{ Absolute effect } & $\begin{array}{l}\text { Relative effect } \\
\qquad(95 \% \mathrm{Cl})\end{array}$ & $\begin{array}{l}\text { No. of } \\
\text { participants } \\
\text { (studies) }\end{array}$ & $\begin{array}{l}\text { GRADE } \\
\text { quality of } \\
\text { evidence }\end{array}$ & Comments \\
\hline $\begin{array}{l}\text { Decreased incidence } \\
\text { of type } 2 \text { diabetes: } \\
\text { FPG, OGTT (mean } \\
\text { follow-up } 3.8 \mathrm{yr} \text { ) }\end{array}$ & 471 per 1000 & $\begin{array}{c}245 \text { fewer per } 1000 \\
(169 \text { to } 254 \text { fewer } \\
\text { per } 1000)\end{array}$ & $\begin{array}{c}\text { RR } 0.48 \\
(0.4-0.58)\end{array}$ & $\begin{array}{c}5275 \\
(5)\end{array}$ & High*†‡ & $\begin{array}{l}\text { NNT } 5 \\
(4-6)\end{array}$ \\
\hline
\end{tabular}

Note: $\mathrm{Cl}=$ confidence interval; FPG = gasting plasma glucose; GRADE = Grading of Recommendations Assessment, Development and Evaluation; NNT = number needed to treat; OGTT = oral glucose tolerance test; $\mathrm{RR}=$ risk ratio.

*Test for heterogeneity: $Q=6.104, p=0.192$.

tThese studies applied to our population group (i.e., age $>35 \mathrm{yr}$, Canadian, ethnic groups).

$\ddagger$ There were sufficient participants, and most events recorded were minimal in the intervention group compared with the control group.

Table 16C: Summary of findings for intensive antihypertensive treatment in patients with type 2 diabetes mellitus to prevent cardiovascular disease

Patient or population: Patients with type 2 diabetes and hypertension

Settings: Hospital outpatient or community health clinics

Intervention: Intensive blood pressure control

Comparison: Moderate blood pressure control

Source: Turnbull F, Neal B, Algert C, et al. Effects of different blood pressure-lowering regimens on major cardiovascular events in individuals with and without diabetes mellitus: results of prospectively designed overviews of randomized trials. Arch Intern Med 2005; 165:1410-9. . $22^{2}$

\begin{tabular}{|c|c|c|c|c|c|c|}
\hline $\begin{array}{l}\text { Outcome* (mean } \\
\text { follow-up } 166.9 \mathrm{yr} \text { ) }\end{array}$ & \multicolumn{2}{|c|}{ Absolute effect } & $\begin{array}{l}\text { Relative effect } \\
\quad(95 \% \mathrm{Cl})\end{array}$ & $\begin{array}{l}\text { No. of } \\
\text { participants } \\
\text { (studies) }\end{array}$ & $\begin{array}{l}\text { GRADE } \\
\text { quality of } \\
\text { evidence }\end{array}$ & $\begin{array}{c}\text { Comments } \\
(95 \% \mathrm{Cl})\end{array}$ \\
\hline $\begin{array}{l}\text { Decrease in } \\
\text { incidence of stroke }\end{array}$ & 46 per 1000 & $\begin{array}{l}17 \text { fewer per } 1000 \\
(9 \text { to } 29 \text { fewer } \\
\text { per } 1000)\end{array}$ & $\begin{array}{c}\text { RR } 0.64 \\
(0.46-0.89)\end{array}$ & $\begin{array}{l}3599 \\
(27)\end{array}$ & Moderate†‡ & $\begin{array}{l}\text { NNT } 61 \\
(41-198)\end{array}$ \\
\hline $\begin{array}{l}\text { Decrease in } \\
\text { cardiovascular events }\end{array}$ & 140 per 1000 & $\begin{array}{l}35 \text { fewer per } 1000 \\
(15 \text { to } 62 \text { fewer } \\
\text { per } 1000)\end{array}$ & $\begin{array}{c}\text { RR } 0.75 \\
(0.61-0.94)\end{array}$ & $\begin{array}{l}3599 \\
(27)\end{array}$ & Moderate†‡ & $\begin{array}{l}\text { NNT } 29 \\
(19-120)\end{array}$ \\
\hline $\begin{array}{l}\text { Decrease in } \\
\text { cardiovascular death }\end{array}$ & 64 per 1000 & $\begin{array}{l}21 \text { fewer per } 1000 \\
\text { (4 to } 52 \text { fewer } \\
\text { per } 1000)\end{array}$ & $\begin{array}{c}\text { RR } 0.67 \\
(0.40-1.12)\end{array}$ & $\begin{array}{l}3599 \\
(27)\end{array}$ & Low†‡§ & $\begin{array}{l}\text { NNT not } \\
\text { statistically } \\
\text { significant }\end{array}$ \\
\hline
\end{tabular}

Note: $\mathrm{Cl}=$ confidence interval; GRADE = Grading of Recommendations Assessment, Development and Evaluation; NNT = number needed to treat; RR = risk ratio. *Definitions from the International Classification of Disease, Ninth Revision.

tHeterogeneity was not indicated for the studies involving diabetic patients; reported heterogeneity value is for all studies, including patients with and without diabetes.

¥The population of interest would consist of people at least 35 years of age, whereas the mean age of participants in these studies was 59.6 to 60 years of age. Additionally, most study participants with diabetes might have been diagnosed many years ago and be symptomatic, whereas our clinical action would involve screening patients who are asymptomatic.

§Wide confidence interval including null effect. 
with fasting glucose or hemoglobin $\mathrm{A}_{1 \mathrm{C}}$ in adults who are overweight or obese (body mass index $\geq 25 \mathrm{~kg} / \mathrm{m}^{2}$ ) and who have one additional risk factor (e.g., physical inactivity, firstdegree relative with diabetes, members of high-risk ethnic populations, hypertension). The International Diabetes Federation guidelines ${ }^{518}$ do not recommend universal screening but suggest that detection programs should target high-risk people identified by assessment of risk factors. Our recommendations focus on improving the precision of screening for high risk ethnic populations.

\section{Take-home messages}

- Persons of South Asian, Latin American and African origin develop hyperglycemia at a younger age and face a twofold to fourfold higher prevalence of type 2 diabetes than white people.

- Persons with hypertension and hypercholesterolemia are at high risk for complications from diabetes and have the most to benefit from treatment of obesity, high cholesterol, hypertension and hyperglycemia.

- Culturally appropriate diabetes education and lifestyle interventions are more effective at controlling levels of hemoglobin $\mathrm{A}_{1 \mathrm{C}}$ than standard approaches.

For the complete evidence review for diabetes in immigrant populations, see Appendix 14, available at www.cmaj.ca/lookup /suppl/doi:10.1503/cmaj.090313/-/DC1. 


\section{Iron-deficiency anemia}

Iron-deficiency is the most common cause of anemia, and iron-deficiency anemia is the most common nutritional disorder in the world. ${ }^{527}$ Other causes of anemia (such as malaria and hemoglobinopathies) may coexist, depending on patients' diets, living conditions and genetic predispositions..$^{59} \mathrm{We}$ focus here on iron-deficiency anemia, which can lead to poor pregnancy outcomes ${ }^{527}$ impaired physical ${ }^{528}$ and cognitive development $^{529}$ in children and reduced work productivity in women. ${ }^{530}$ No routine iron-deficiency screening or supplementation program is offered in Canada for immigrants, either before or after their arrival. We conducted an evidence review on the effectiveness of screening and iron-supplement interventions for immigrant populations. The recommendations of the Canadian Collaboration for Immigrant and Refugee Health on screening for and treatment of iron-deficiency anemia are outlined in Box 17A.

\section{Methods}

We used the 14-step method developed by the Canadian Collaboration for Immigrant and Refugee Health ${ }^{16}$ (summarized in section 3 of this article, above). We considered the epidemiology of iron-deficiency anemia in immigrant populations and defined clinical preventive actions (interventions), outcomes and key clinical questions. We searched MEDLINE, Embase, CINAHL, the Cochrane Library and other sources from Jan. 1, 1996, to Jan. 1, 2010. Detailed methods, search terms, case studies and clinical considerations can be found in the complete evidence review for iron-deficiency anemia (Appendix 15, available at www.cmaj.ca/lookup/suppl/doi:10.1503/cmaj .090313/-/DC1).

\section{Results}

We found no systematic reviews or guidelines on screening for iron-deficiency anemia among immigrants and refugees. We based our search update on the US Preventive Services Task Force's systematic review on iron-deficiency anemia. ${ }^{531}$ We identified relevant clinical trials that would help assess the effectiveness of screening for and treating iron-deficiency anemia $^{530,532}$ and that added new and primary evidence to the published systematic reviews. ${ }^{528,529,533}$ We identified 76 articles as relevant to screening and treatment of iron-deficiency anemia among immigrants and refugees.

\section{What is the burden of iron-deficiency anemia in immigrant populations?}

Newly arriving immigrant and refugee children and women have a higher prevalence of anemia $(15 \%-28 \%)^{60}$ than the Canadian-born population (2\%-10\%), excluding First Nations populations. The World Health Organization has estimated that the prevalence of iron-deficiency anemia among preschool children ranges from $21 \%$ to $68 \% .^{59}$ The prevalence of iron-deficiency anemia for women of reproductive age ranges from $18 \%$ to $48 \%$ (Table 17A). ${ }^{59}$

The main risk factors for iron-deficiency anemia are low iron intake, poor absorption of iron related to diets high in phytate or phenolic compounds, and periods of life with high iron demand. ${ }^{59}$ Studies on newly arriving refugee children have identified exclusive breastfeeding after six months of age without the use of iron supplements or iron-rich complemen-

\section{Box 17A: Recommendations from the Canadian Collaboration for Immigrant and Refugee Health: iron-deficiency anemia \\ Women}

Screen immigrant and refugee women of reproductive age for iron-deficiency anemia (with hemoglobin).

\section{Basis of recommendation}

\section{Balance of benefits and harms}

Treating iron-deficiency anemia provides an average net change in hemoglobin concentration of $15 \mathrm{~g} / \mathrm{L}$ (number needed to treat [NNT] 2, 95\% confidence interval [Cl] 2-3) and an increase in function and provides a net change in the productivity ratio (NNT 4, 95\% Cl 3-8). The prevalence of iron deficiency is higher among immigrant women than among Canadian-born women (>15\% v. $<15 \%)$. Harms are minimal and include diarrhea and the personal costs of iron supplements.

\section{Quality of evidence}

Moderate

\section{Values and preferences}

The committee attributed more value to improving health among women of child-bearing age and less value to uncertainty about whether asymptomatic immigrant and refugee women value the treatment outcomes.

\section{Children}

Screen immigrant and refugee children aged one to four years for iron-deficiency anemia (with hemoglobin).

\section{Basis of recommendation}

\section{Balance of benefits and harms}

Treating children with iron-deficiency anemia improves cognitive development, with a standardized mean difference of 0.30 , equivalent to a modest effect of 1.5-2 intelligence quotient points (NNT 7, 95\% Cl 5-14). Immigrant and refugee children have a higher prevalence of iron-deficiency anemia than Canadian-born children ( $>20 \%$ v. $<20 \%$ ). Adverse effects from iron treatment are minimal. The NNT for immigrant and refugee children is expected to be similar because many of the studies were conducted in developing countries.

\section{Quality of evidence}

Moderate

\section{Values and preferences}

The committee attributed more value to ensuring optimal opportunities for immigrant children and potential reduction of disparities in education, literacy and wages between immigrant and Canadian-born populations and less value to the discomfort of testing and treatment risk of diarrhea. 
tary foods, use of cow's milk or nonfortified infant formula as weaning food, early and frequent consumption of tea and relatively infrequent consumption of meat. ${ }^{534}$ Other important considerations in children are the presence of other illnesses ${ }^{527}$ and obesity. ${ }^{535}$
Migrants from regions in the developing world with a high prevalence of hookworm and malaria, ${ }^{536}$ high parity and genetic predisposition for red blood cell disorders (hemoglobinopathies) are at increased risk for anemia. Alpha- and betathalassemias are most common in Africa, the Mediterranean,

Table 17A: Anemia prevalence and number of people affected among preschool-age children and nonpregnant women in each World Health Organization (WHO) region*

\begin{tabular}{|c|c|c|c|c|}
\hline \multirow[b]{2}{*}{ WHO region } & \multicolumn{2}{|c|}{ Preschool-age children $\dagger$} & \multicolumn{2}{|c|}{ Nonpregnant women } \\
\hline & $\begin{array}{l}\text { Prevalence, } \% \\
\quad(95 \% \mathrm{Cl})\end{array}$ & $\begin{array}{c}\text { No. affected, millions } \\
(95 \% \mathrm{Cl})\end{array}$ & $\begin{array}{l}\text { Prevalence } \\
(95 \% \mathrm{Cl})\end{array}$ & $\begin{array}{l}\text { No. affected, millions } \\
(95 \% \mathrm{Cl})\end{array}$ \\
\hline Africa & $67.6(64.3-71.0)$ & $83.5 \quad(79.4-87.6)$ & $47.5(43.4-51.6)$ & $69.9 \quad(63.9-75.9)$ \\
\hline Americas & $29.3(26.8-31.9)$ & $23.1 \quad(21.2-25.1)$ & $17.8(12.9-22.7)$ & $39.0 \quad(28.3-49.7)$ \\
\hline Southeast Asia & $65.5(61.0-70.0)$ & $115.3(107.3-123.2)$ & 45.7 (41.9-49.4) & $182.0(166.9-197.1)$ \\
\hline Europe & 21.7 (15.4-28.0) & $11.1 \quad(7.9-14.4)$ & $19.0(14.7-23.3)$ & $40.8 \quad(31.5-50.1)$ \\
\hline Eastern Mediterranean & $46.7(42.2-51.2)$ & $0.8 \quad(0.4-1.1)$ & $32.4(29.2-35.6)$ & $39.8 \quad(35.8-43.8)$ \\
\hline Western Pacific & $23.1(21.9-24.4)$ & $27.4 \quad(25.9-28.9)$ & $21.5(20.8-22.2)$ & $(94.0-100.0)$ \\
\hline Global & $47.4(45.7-49.1)$ & $293.1(282.8-303.5)$ & $30.2(28.7-31.6)$ & 468.4 (446.2-490.6) \\
\hline
\end{tabular}

Note: $\mathrm{Cl}=$ confidence interval.

*Adapted, with permission, from the World Health Organization. ${ }^{59}$

tPreschool-age children: 0.00-4.99 yr.

‡Nonpregnant women: $15.00-49.99 \mathrm{yr}$

Table 17B: Summary of findings for iron supplements to treat iron deficiency in children

Patient or population: Children with iron deficiency

Setting: International locations (developed and developing countries), participants identified within communities or schools

Intervention: Iron supplements

Comparison: Children without iron deficiency

Sources: Sachdev H, Gera T, Nestel P. Effect of iron supplementation on mental and motor development in children: systematic review of randomised controlled trials. Public Health Nutr 2005;8:117-32. ${ }^{529}$ Gera T, Sachdev HP. Effect of iron supplementation on incidence of infectious illness in children: systematic review. BMJ 2002;325:1-10. ${ }^{533}$ Sachdev H, Gera T, Nestel P. Effect of iron supplementation on physical growth in children: systematic review of randomized controlled trials. Public Health Nutr 2006;9: $904-20 .{ }^{528}$

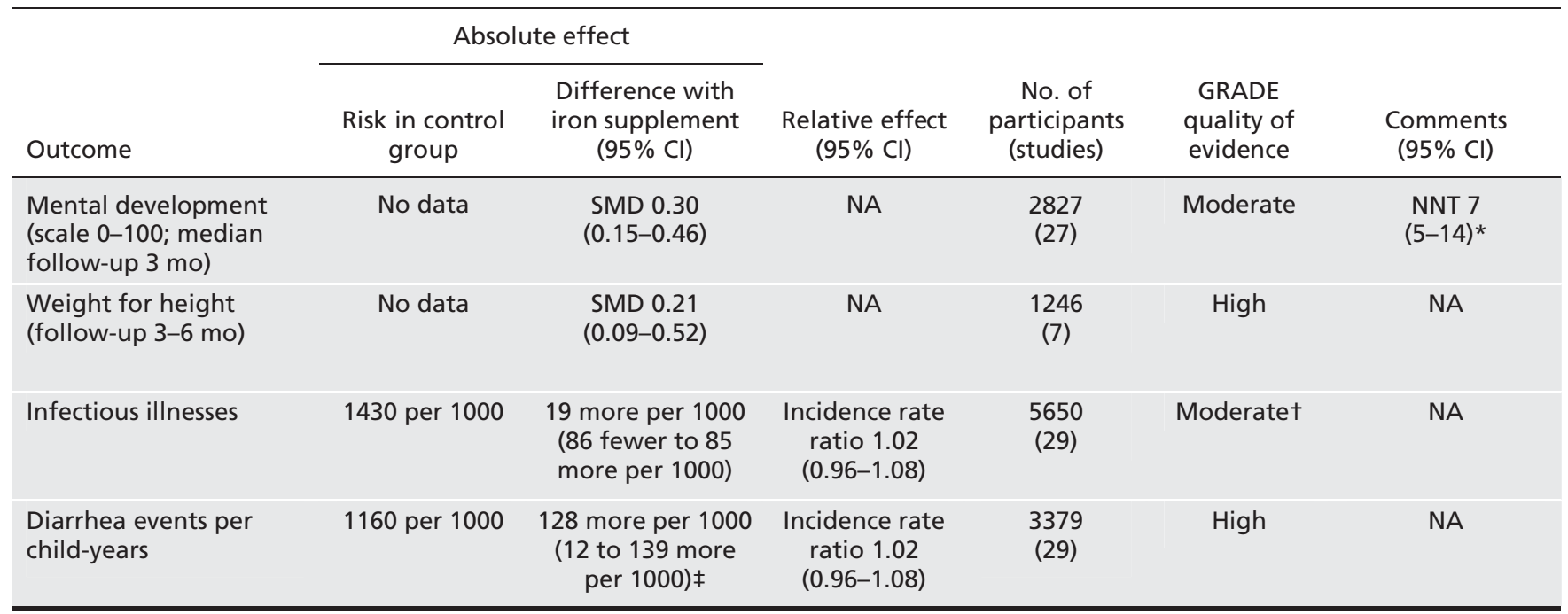

Note: $\mathrm{Cl}=$ confidence interval; GRADE = Grading of Recommendations Assessment, Development and Evaluation; NA = not applicable; NNT = number needed to treat; $\mathrm{SMD}=$ standard mean difference.

*This is a modest effect, equivalent to 1.5-2.0 intelligence quotient points. In younger children (age $\leq 27$ months), no effect of iron supplementation on mental development was detected.

tConsistency in infectious illnesses: heterogeneity $Q=78.29, \mathrm{df}=28, p<0.0001$.

fIntervention and control group numbers are the number of events per child-years (observation/exposure time). 
India and Southeast Asia, whereas sickle cell anemia is most often found in people of African descent. Glucose-6-phosphate deficiency (worldwide equatorial distribution) can lead to hemolysis in relation to oxidative injury from certain medications (e.g., nitrofurantoin, sulfamethoxazole) and thus can contribute to iron deficiency. ${ }^{59}$

\section{Does screening for iron-deficiency anemia decrease morbidity?}

\section{Screening}

The positive predictive value of hemoglobin concentration below $110 \mathrm{~g} / \mathrm{L}$ alone for iron deficiency in children (12-35 months) is $29 \%$ (95\% confidence interval [CI] 20\%-38\%), and the sensitivity is $30 \%$ ( $95 \%$ confidence interval [CI] $20 \%-40 \%){ }^{531}$ Diagnostic measures used to confirm irondeficiency anemia include serum ferritin and hemoglobin or hematocrit response to administration of iron.

\section{Relative benefits and harms of treatment}

Iron deficiency can be addressed through primary prevention by ensuring adequate iron intake $\mathrm{e}^{527,537-539}$ or through secondary prevention by detecting the condition and treating it with iron supplements or diet education. ${ }^{540}$

\section{Cognitive development in children}

Sachdev and colleagues ${ }^{528}$ evaluated changes in weight-forheight between placebo and iron-treatment groups in children and found no statistically significant difference. Sachdev and colleagues $^{529}$ also conducted a meta-analysis combining 17 trials (10 of which were conducted in developing countries) of iron supplementation in infants or in children up to 12 years of age and found increased mental development scores in the treatment group (Table 17B). In the subgroup analyses, improvement in mental development scores was attributed mainly to five trials in children seven years of age or older, and the effect was only intermediate for children between two and five years of age. Common adverse effects associated with iron supplements include dose-related reversible gastrointestinal symptoms ${ }^{531,533}$ and unintentional overdose.

\section{Work productivity in women of reproductive age}

We identified three randomized controlled trials studying the effect of iron supplements on work productivity. ${ }^{530,541,542}$ These trials, conducted in China, Indonesia and Sri Lanka, all reported increases in work productivity with iron supplements. Table 17C presents the study from China, which we chose as the best-quality study for women of child-bearing age who are not pregnant. ${ }^{530}$ Because of the complexity of measuring work productivity and the significance for only one of the two primary measures, we rated the quality of this evidence as low.

\section{Clinical considerations}

Poverty, food insecurity, culture, customs and limited education attainment can affect the choice and availability of ironrich foods. ${ }^{543}$ Many refugees and some immigrants have ingested iron-insufficient diets for an extended period and do not recognize symptoms of iron deficiency. Culturally appropriate nutritional assessment and counselling by a registered dietician, when available, can identify specific nutrition problems and support appropriate change. ${ }^{531}$

\section{What are the potential implementation issues?}

Accessibility issues include language barriers, lower levels of education, low levels of ongoing support and follow-up, and

Table 17C: Summary of findings for iron supplements compared with placebo for women of reproductive age

Patient or population: Women of reproductive age (19-44 yr)

Setting: Factory in China (workers in cotton mill)

Intervention: Oral iron supplementation

Comparison: Placebo

Source: Li R, Chen X, Yan H, et al. Functional consequences of iron supplementation in iron-deficient female cotton mill workers in Beijing, China. Am J Clin Nutr 1994;59:908-13. ${ }^{330}$

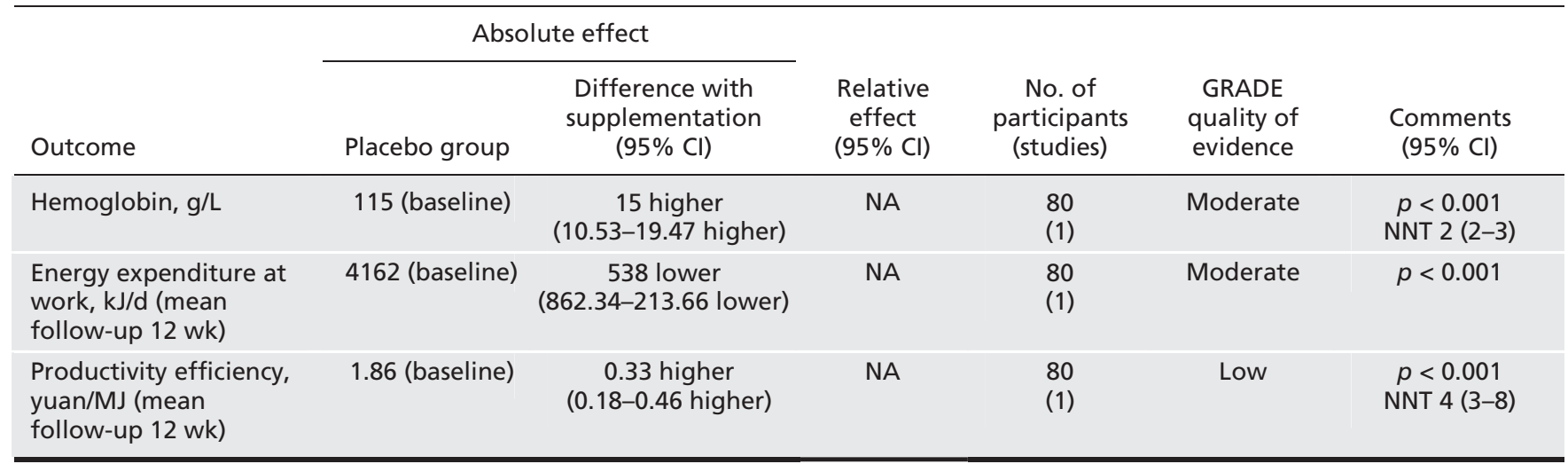

Note: $\mathrm{Cl}=$ confidence interval; GRADE = Grading of Recommendations Assessment, Development and Evaluation; NA = not applicable; NNT = number needed to treat. 
access to supplements. ${ }^{54}$ Gastrointestinal side effects, changes in stool colour and three times daily dosing have been shown to reduce adherence rates. ${ }^{531}$

\section{Recommendations of other groups}

The US Preventive Services Task Force recommends screening for iron-deficiency anemia in children 6-12 months of age and pregnant women who are at risk for iron deficiency. ${ }^{531}$ The US Centers for Disease Control and Prevention recommend routine screening for all children and women of reproductive age, citing the linked risks of iron-deficiency anemia in pregnancy affecting both women and infants. ${ }^{527}$ The World Health Organization recommends screening for at-risk women and children. ${ }^{54}$ Our recommendations highlight the importance of screening for iron-deficiency anemia in immigrant children and women of child-bearing age.

\section{Take-home messages}

- Immigrants and refugees coming from regions with limited access to iron-rich foods, higher rates of infectious disease and higher parity are at risk for iron deficiency.

- To improve their cognitive development, growing children aged one to four years should be screened for iron deficiency by means of hemoglobin measurement.

- To improve hemoglobin levels and work productivity, immigrant and refugee women of reproductive age should also be screened by means of hemoglobin measurement.

For the complete evidence review for iron-deficiency anemia in immigrant populations, see Appendix 15, available at www .cmaj.ca/lookup/suppl/doi:10.1503/cmaj.090313/-/DC1. 


\section{Dental disease}

Dental caries affect virtually $100 \%$ of adults and $60 \%-90 \%$ of children worldwide, and periodontal disease is found in $5 \%-20 \%$ of most adult populations. ${ }^{546}$ Both are preventable chronic infectious diseases influenced by sociobehavioural, economic and environmental risk factors. In addition to oral pain, infection, tooth loss and associated dysfunction, chronic oral conditions can have a profound effect on general health and quality of life. Pain and disability associated with poor oral health can compromise a person's ability to eat properly, which affects nutrition status and body weight of both children and older adults. ${ }^{546}$ We conducted an evidence review to guide primary care practitioners in the early detection, prevention and treatment of common oral conditions for newly arriving immigrants. The recommendations of the Canadian Collaboration for Immigrant and Refugee Health on screening for and treatment of dental and periodontal disease are found in Box 18A.

\section{Methods}

We used the 14-step method developed by the Canadian Collaboration for Immigrant and Refugee Health ${ }^{16}$ (summarized in section 3 of this article, above). We considered the epidemiology of oral disease in immigrant populations and defined clinical preventive actions (interventions), outcomes and key clinical questions. We searched MEDLINE, Embase, CINAHL, HealthSTAR, the Cochrane Library and other sources from Jan. 1, 1996, to Jan. 1, 2010. Detailed methods, search terms, case studies, clinical considerations and research needs can be found in the complete evidence review for dental disease (Appendix 16, available at www.cmaj.ca/lookup/suppl /doi:10.1503/cmaj.090313/-/DC1).

\section{Results}

We found no systematic reviews or evidence-based guidelines specific to immigrants and refugees. The next search produced 35 relevant manuscripts, and five high-quality systematic reviews met the inclusion criteria. ${ }^{61,62,547-549}$ The focused guideline searches resulted in five relevant evidence-based, consensus-driven guidelines. The search for preventive and restorative interventions for dental referral yielded 10 high-quality systematic reviews. ${ }^{550-599}$ Fifty-six articles addressing prevalence, burden of disease, disease risk and access to care in immigrant populations were selected for detailed review.

\section{What is the burden of oral disease in immigrant populations?}

Immigrant adolescent children were five times more likely to have dental caries than children born in Canada, and 22.9\% required restorative dental care for carries, compared with only $3.5 \%$ of those born in Canada. ${ }^{560}$ Although levels of oral disease in immigrants decreased relative to length of time in
Canada, immigrant adolescents continued to be at a disadvantage for dental caries, gingivitis and level of oral hygiene when compared with their Canadian counterparts.

Epidemiologic information from the World Health Organization shows that development of caries is on the rise in developing countries in Africa and Asia. The increased consumption of refined sugar and inadequate exposure to the topical fluorides available in toothpastes and professionally applied fluoride products available in developed nations contribute to high rates of disease. ${ }^{546}$

\section{Does screening decrease morbidity from common oral diseases?}

\section{Screening}

The mouth should be examined using a tongue depressor to determine swelling, bleeding gums, loose teeth, broken teeth or holes in teeth, odour, mouth ulcers and sores. A systematic review and randomized controlled trial provide evidence that

\section{Box 18A: Recommendations from the Canadian Collaboration for Immigrant and Refugee Health: dental disease}

Screen all immigrants for dental pain (asking, "Do you have any problems or pain with your mouth, teeth or dentures?"). Treat dental pain with nonsteroidal antiinflammatory drugs (NSAIDs) and refer patients to a dentist.

Screen all immigrant children and adults for obvious dental caries and oral disease (examine mouth with penlight and tongue depressor). Refer patients with obvious dental disease to a dentist or oral health specialist.

\section{Basis of recommendation}

\section{Balance of benefits and harms}

Screening and treating dental pain led to a substantial decrease in pain and swelling (number needed to treat [NNT] 34, 95\% confidence interval [Cl] not estimable). Screening and referring patients for treatment of dental disease led to a significant decrease in dental caries (NNT $2.9,95 \% \mathrm{Cl} 2.1-3.4$ ). Given the higher prevalence of dental caries in new immigrants (adolescents: $23 \%$ v. $3.5 \%$ of Canadian-born), the number needed to screen and NNT for net benefits are expected to be lower, despite potential issues affecting access to care. Harms of pain control are minimal and included adverse events from short-term NSAIDs. Harms of referral included patient-borne costs and discomfort or anxiety.

\section{Quality of evidence}

Moderate

\section{Values and preferences}

The committee attributed more value to reducing dental pain and less value to the small risk of adverse gastrointestinal effects with NSAID therapy. For referrals, the committee attributed more value to reducing oral health disparities in immigrant communities and less value to burden of screening and potential costs of dental care for patients. 
physicians can screen preschool children for dental caries with a high degree of accuracy. ${ }^{62}$ The evidence supports the effectiveness of prevention through application of sealants in children (Table 18A). ${ }^{557}$

\section{Relative benefits and harms of treatment}

The management of underlying causes of dental pain by dentists is effective for prevention and management of dental disease. ${ }^{548}$ Potential harms include the cost to patients to access dental care and adverse reactions to treatment. Nonsteroidal anti-inflammatory drugs (NSAIDs) manage oral pain effectively (Table 18B). ${ }^{548}$ Antibiotics should be prescribed only in the presence of concomitant systemic symptoms, such as lymphadenopathy, fever, and associated cellulitis. ${ }^{561}$ Singledose studies showed no difference in short-term adverse effects between oral ibuprofen and placebo. ${ }^{562}$ In patients given NSAIDs over longer periods, adverse reactions included abdominal pain, diarrhea, edema, dry mouth, rash, dizziness, headache and fatigue. These are generally considered to be mild to moderately severe. ${ }^{563}$

\section{Clinical considerations}

\section{What are potential implementation issues?}

Lower income and immigrant status are both associated with fewer visits for preventive dental care. ${ }^{564}$ Language barriers reduce access to services and quality of care. ${ }^{565}$ Experiential influences (fear of dentists, history of inadequate care, embarrassment about oral condition) are likely hindrances for individuals who require professional dental care. Financial barriers decrease access to dental care. When nondentists refer on the basis of an oral health screening, people are twice as likely to go to the dentist. ${ }^{62}$ However, active referral (e.g., specific clinics identified, a patient information notice) is warranted. ${ }^{566}$

\section{Financial coverage of dental care}

During the settlement period, Convention Refugees, refugee claimants and other protected people are eligible to apply for Interim Federal Health Program coverage for dental care. Details of services covered, the application process and a hand-

Table 18A: Summary of findings for use of sealant to prevent carious lesions

Patient or population: Children and adolescents (aged 6-19 yr) with carious lesions on permanent teeth

Setting: Dental clinics in Brazil, United States, Canada, Zimbabwe

Intervention: Sealants

Comparison: No sealant

Source: Griffin SO, Oong E, Kohn W, et al.; CDC Dental Sealant Systematic Review Work Group. The effectiveness of sealants in managing caries lesions. J Dent Res 2008;87:169-74..$^{557}$

\begin{tabular}{|c|c|c|c|c|c|c|}
\hline Outcome & \multicolumn{2}{|c|}{ Absolute effect } & $\begin{array}{l}\text { Relative } \\
\text { effect } \\
(95 \% \mathrm{Cl})\end{array}$ & $\begin{array}{l}\text { No. of } \\
\text { participants } \\
\text { (studies) }\end{array}$ & $\begin{array}{l}\text { GRADE } \\
\text { quality of } \\
\text { evidence }\end{array}$ & $\begin{array}{c}\text { Comments } \\
(95 \% \mathrm{Cl})\end{array}$ \\
\hline Progressing lesions & 480 per 1000 & $\begin{array}{l}350 \text { fewer per } 1000 \\
(345 \text { to } 168 \text { fewer } \\
\text { per } 1000)\end{array}$ & $\begin{array}{c}\text { RR } 0.27 \\
(0.01-0.38)\end{array}$ & $\begin{array}{l}200 \\
(4)\end{array}$ & High & $\begin{array}{l}\text { NNT } 2.9 \\
(2.1-3.4)\end{array}$ \\
\hline
\end{tabular}

Note: $\mathrm{Cl}=$ confidence interval; GRADE = Grading of Recommendations Assessment, Development and Evaluation; NNT = number needed to treat; RR = risk ratio.

Table 18B: Summary of findings for use of NSAIDS as treatment for pain and swelling of dental origin

Patient or population: Adults with pain (without localized swelling) of dental origin and no systemic symptoms (such as fever or lymphadenopathy)

Setting: Dental offices and hospitals in United States, United Kingdom

Intervention: NSAIDs

Comparison: Placebo

Source: Sutherland SE, Matthews DC. Emergency management of acute apical periodontitis in the permanent dentition: a systematic review of the literature. J Can Dent Assoc 2003; 69:160. ${ }^{548}$

\begin{tabular}{|c|c|c|c|c|c|c|}
\hline Outcome & \multicolumn{2}{|c|}{ Absolute effect } & $\begin{array}{l}\text { Relative effect } \\
\quad(95 \% \mathrm{Cl})\end{array}$ & $\begin{array}{l}\text { No. of } \\
\text { participants } \\
\text { (studies) }\end{array}$ & $\begin{array}{l}\text { GRADE } \\
\text { quality of } \\
\text { evidence }\end{array}$ & $\begin{array}{c}\text { Comments } \\
(95 \% \mathrm{Cl})\end{array}$ \\
\hline $\begin{array}{l}\text { Mean pain relief } \\
\text { (100-mm visual analogue } \\
\text { scale) at } 24 \mathrm{~h}\end{array}$ & NA & NA & $\begin{array}{l}\text { Weighted mean } \\
\text { difference } 22.7 \\
(-36.2 \text { to }-9.21)\end{array}$ & $\begin{array}{l}619 \\
(6)\end{array}$ & High & $\begin{array}{c}\text { NNT } 35 \\
(95 \% \mathrm{Cl} \text { not } \\
\text { estimable)* }\end{array}$ \\
\hline
\end{tabular}

Note: $\mathrm{Cl}=$ confidence interval; GRADE = Grading of Recommendations Assessment, Development and Evaluation; NA = not applicable; NSAID = nonsteroidal anti-

*Data in source article were insufficient to allow estimation of $95 \% \mathrm{Cl}$. 
book for health care providers are available through the Citizenship and Immigration Canada website (www.cic.gc.ca). In addition to the provincial public dental programs, there are low-cost or no-cost clinics in several of the larger cities. Information on many of these programs and clinics is available from the website of the Federal, Provincial and Territorial Dental Working Group (www.fptdwg.ca) and the Canadian Association of Public Health Dentistry (www.caphd-acsdp.org). In the absence of comprehensive dental services for marginalized populations, physicians' involvement in oral health care is increasing ${ }^{567}$ and the application of fluoride varnish by primary care practitioners shows great promise. ${ }^{568}$

\section{Recommendations of other groups}

The Canadian Collaboration for Clinical Practice Guidelines in Dentistry, in a 2003 guideline, suggested that the most efficacious method to reduce tooth pain with or without localized swelling (when dental therapy cannot be started immediately) is with NSAIDs ${ }^{561}$ Other guideline development groups have reported moderate- to high-quality evidence to support recommendations for the application of fluoride varnish to teeth of children at high risk for caries and the recommendation that teeth be brushed twice daily with toothpaste containing 1000 ppm fluoride. ${ }^{569,570}$ Our recommendations focus on screening all immigrants for dental pain and highlight the benefit of practitioner referrals for those with dental pathology.

\section{Take-home messages}

- Dental pain can be reduced if physicians ask whether patients have problems with their mouth, teeth or dentures. NSAIDs can be used effectively to treat dental pain.

- Migrants arriving from countries with limited dental care and where diets are high in sugar are at the highest risk for disease.

- Screening and referral for dental disease can facilitate treatment and prevention of dental disease.

- Patients are twice as likely to go for dental treatment when they are actively examined and referred by a physician.

- Tooth-brushing twice daily with fluoridated toothpaste is effective in reducing the risk of dental decay.

For the complete evidence review for dental disease in immigrant populations, see Appendix 16, available at www.cmaj.ca/lookup /suppl/doi:10.1503/cmaj.090313/-/DC1. 


\section{Vision health}

Together, blinding disorders are the seventh leading cause of burden of disease, ahead of diabetes and cancer. ${ }^{571,572}$ Vision loss can limit the ability to work, drive and complete other activities of daily living. Even modest visual impairment (visual acuity $<6 / 12$ ) is associated with substantial morbidity..$^{573-575}$ Uncorrected refractive error is the leading cause of visual impairment worldwide. ${ }^{576}$ Vision loss from most of these conditions is largely preventable with timely diagnosis and treatment. Even age-related macular degeneration is yielding somewhat to interventions. ${ }^{577.578}$ However, access to even basic vision care is often limited in developing countries. ${ }^{579}$ In light of evidence that source countries for new immigrants and refugees to Canada have higher burdens of vision loss, ${ }^{63}$ we reviewed evidence on prevalence, screening of asymptomatic populations, treatment effectiveness, population-specific concerns and implementation. The recommendations of the Canadian Collaboration for Immigrant and Refugee Health on vision health are outlined in Box 19A.

\section{Methods}

We used the 14-step method developed by the Canadian Collaboration for Immigrant and Refugee Health ${ }^{16}$ (summarized in section 3 of this article, above). We defined clinical preventive actions (interventions) and relevant clinical outcomes, concentrating on the body of evidence for screening tests for vision loss. We searched MEDLINE, Embase, CINAHL, the Cochrane Library and other sources from Jan. 1, 1996, to Jan. 1, 2010. Detailed methods, search terms and references, case studies and clinical considerations can be found in the complete evidence review for vision health (Appendix 17, available at www.cmaj.ca/lookup/suppl/doi:10.1503/cmaj.090313 /-/DC1).

\section{Results}

Our search did not yield any systematic reviews or guidelines related to vision screening tests in immigrants or refugees. However, we identified 23 systematic reviews and guidelines related to screening tests for vision loss in the general population. Our review identified four clinical trials of screening tests for vision loss in community-dwelling elderly people. ${ }^{580}$ These studies demonstrated no benefit, but none of them could be generalized to newly arrived immigrants and refugees. Eight guidelines for adults and one article focusing on amblyopia in children were identified; all recommended vision screening tests in varying forms and frequency.

\section{What is the burden of vision loss in immigrant populations?}

Visual acuity is the single most important indicator of ocular health. Decreased presenting vision is most frequently due to uncorrected refractive error but can signify underlying sightthreatening eye disease. International data ${ }^{63}$ suggest that vision loss and undiagnosed sight-threatening eye disease should be more common in new immigrants and refugees to Canada who originate from developing countries, but we could not find any Canadian data to confirm this assertion. Much of the high prevalence of blindness in developing countries can be attributed to disparities in access to care. ${ }^{579}$ The World Health Organization has estimated that $80 \%$ of blindness in developing countries could be avoided through cost-effective prevention and treatment. Glaucoma, the leading cause of irreversible blindness worldwide,${ }^{63}$ reflects the global disparity in access to care, with only $10 \%$ of cases of open-angle glaucoma diagnosed in developing countries compared with $50 \%$ in developed countries. ${ }^{581}$ Similar global disparities in access to care are anticipated for other common causes of vision loss. ${ }^{579}$

In the United States, minority populations have higher levels of vision loss and undiagnosed eye disease ${ }^{582-585}$ than does the general population. Numerous factors contribute to this disparity, including socioeconomic status, access to care, societal conventions, and physiologic and anatomic differences. ${ }^{54}$ These factors, in turn, influence the prevalence of vision loss from cataract, diabetic retinopathy, glaucoma and

\section{Box 19A: Recommendations from the Canadian Collaboration for Immigrant and Refugee Health: vision health}

Perform age-appropriate screening for visual impairment.* If presenting vision $<6 / 12$ (with habitual correction in place), refer patients to an optometrist or ophthalmologist for comprehensive ophthalmic evaluation.

\section{Basis of recommendation}

\section{Balance of benefits and harms}

Uncorrected refractive error, the most common cause of visual impairment, is amenable to correction with eyeglasses (number needed to screen to find one person with vision worse than $6 / 15$ or $20 / 50$ due to uncorrected refractive error $=19$ ). Prevalence of uncorrected refractive error in immigrant populations is higher than in the general population; however, economic and cultural barriers could reduce rates of referral and use of corrective eyeglasses. Harms are minimal and can include out-of-pocket costs.

\section{Quality of evidence}

Very low

\section{Values and preferences}

The committee attributed more value to ensuring that visual acuity is adequate for daily functioning and employment and to detecting serious underlying ocular disease. The committee attributed less value to the burden of screening and the cost of eyeglasses.

*Visual acuity should be measured with distance glasses or contact lenses in place if worn habitually. Age-appropriate measurement in children is required at 0-3 months (infant should react to light), at 6-12 months (baby's eyes should fix and follow light) and at 3-5 years (child should use visual acuity chart where possible). Additional screening manoeuvres are useful for children: at each screening interval, assess for red reflex and inspect external ocular structures. For patients 6 months and older, also assess for strabismus. 
uncorrected refractive error. ${ }^{584,586-588}$ Recent immigration $^{582,583}$ has been identified as a risk factor for vision loss, the most common cause of which is uncorrected refractive error..$^{52,585,589}$ The prevalence of vision loss $(<6 / 15)$ due to uncorrected refractive error is estimated at $5.4 \%$ for those 12 and older in the general population and substantially higher in minority populations. $^{589}$

Although trachoma, onchocerciasis (river blindness) and vitamin A deficiency figure prominently as causes of blindness in some low-income countries, these conditions have not been reported among immigrants and refugees in Canada. Anecdotal evidence suggests that the rare cases in immigrants and refugees to Canada most frequently take the form of subconjunctival scarring from cicatricial trachoma (noninfectious) evident on eversion of the upper eyelids. Typically, the threat to vision posed by these conditions in asymptomatic patients dissipates harmlessly on arrival to Canada through a vitamin A-sufficient diet or by breaking the cycle of re-infection that underlies the decades-long descent into blindness from trachoma or onchocerciasis.

\section{Do screening tests and treatment for eye disease decrease morbidity?}

\section{Screening}

Measuring visual acuity is a simple manoeuvre that can be carried out accurately (sensitivity of $94 \%$ and specificity of $89 \%)^{590}$ in a primary care setting. When measuring vision in immigrants and refugees, a standardized chart with the tumbling $\mathrm{E}$ or the Landholt $\mathrm{C}$ optotypes could be helpful, because familiarity with an alphabet or numbers is not required. In some cases, a cut-out "E" or "C" for the reader to orient to the chart will simplify communication. The ETDRS-type of visual acuity chart is the preferred chart layout and has several advantages, including five optotypes on each line. ${ }^{591}$ However, an inexpensive Snellen chart, which uses ambient room light- ing, is adequate for screening purposes. A pinhole viewing device can be added as a simple adjunct to determine whether the visual impairment is due to refractive error (sensitivity $79 \%$, specificity $98 \%) .{ }^{592}$

\section{Relative benefits and harms of treatment}

We did not find direct evidence that routinely screening and treating immigrant children in primary care for visual impairment results in improved visual acuity. However, a randomized clinical trial has shown that intensive screening procedures, compared with usual vision surveillance, leads to improved visual acuity. ${ }^{593}$ Amblyopia and its leading causes (strabismus, astigmatism and anisometropia) are reversible if diagnosed and treated early after onset within the first seven or eight years of life, after which reversibility is minimal.

For adults, we focused our effectiveness synthesis on the prevalence of vision loss from uncorrected refractive error, which is amenable to simple correction with eyeglasses. ${ }^{589} \mathrm{We}$ were unable to identify any evidence to estimate the effect of adherence to recommendations for an eye examination on whether subsequent prescriptions for eyeglasses are filled. Using the GRADE rating system, we rated the quality of this evidence as very low. Our evidence review found no data on harm to the patient for measuring vision (Table 19A).

\section{Clinical considerations}

\section{What are the potential implementation issues?}

Comprehensive ophthalmic examination is not covered for those aged 18 to 64 in any Canadian province. ${ }^{594}$ However, specific nonrefractive indications for performing an evaluation - such as being at increased risk for glaucoma, screening for diabetic retinopathy or excluding clinically evident cataract as a cause of vision loss - are typically covered by provincial health plans. Convention refugees, covered under the Interim Federal Health Program, receive coverage for cor-

Table 19A: Summary of findings for vision screening and correction to reduce visual impairment

Patient or population: General US population, age $\geq 12 \mathrm{yr}$

Setting: Household study of adolescents and adults

Intervention: Screening and correction of visual impairment

Comparison: Usual care

Source: Vitale S, Cotch MF, Sperduto RD. Prevalence of visual impairment in the United States. JAMA 2006;295:2158-63. ${ }^{589}$

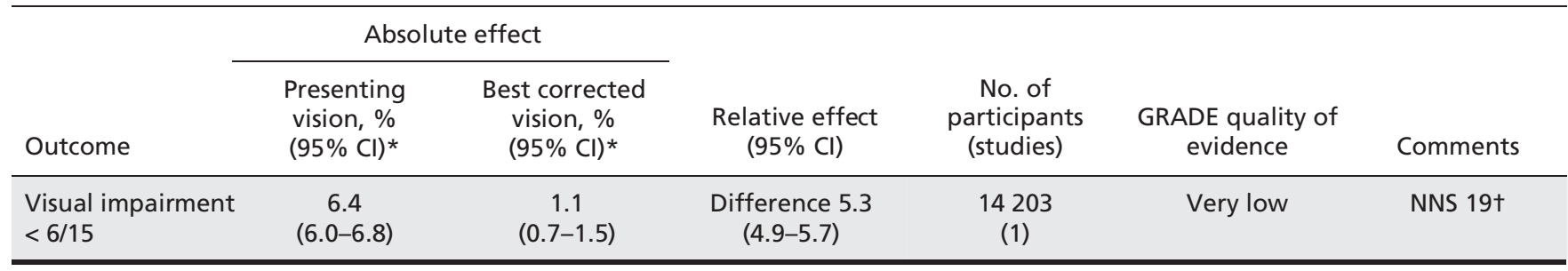

Note: $\mathrm{Cl}=$ confidence interval; GRADE = Grading of Recommendations Assessment, Development and Evaluation; NNS = number need to screen to find one person with vision worse than $6 / 15$ or $20 / 50$ because of uncorrected refractive error.

*Presenting visual impairment is based on vision with habitual correction, if worn, in place. Best-corrected visual impairment is based on vision with best possible refractive correction in place.

†Acceptance of correction through eyeglasses can only be inferred. The study was a large population-based examination survey, not a treatment study. Proportion of participants who would benefit from correction using eyeglasses was estimated by the change in prevalence of visual impairment based on refraction compared with presenting vision. In practice, the NNS to improve vision would be greater because not everyone whose vision would improve to better than $20 / 50$ would accept the cost and inconvenience of eyeglasses. 
rection with eyeglasses and other urgent care for up to one year after arrival.

Beyond issues of financial access, age and sex can be influential. Age can influence the decision to seek care or to treat, a decision that is sometimes beyond the control of elderly patients with vision loss. Female patients appear to face greater barriers to assessment and treatment, and sex discrimination is more influential in low- and middle-income countries than in high-income countries. ${ }^{595}$ Finally, stigma associated with wearing glasses can also influence eye care for refugees and immigrants to Canada. The desire to conform with societal norms and beauty standards is often stronger than the desire for treatment. ${ }^{596}$

\section{Recommendations of other groups}

The recommendations of other groups regarding vision health are presented below. Our recommendations highlight the importance of screening all immigrants for visual impairment.

\section{Screening tests for visual impairment in children}

The Canadian Task Force on the Periodic Health Examination $^{597,598}$ (now the Canadian Task Force on Preventive Health Care) and the US Preventive Services Task Force recommend screening tests for visual impairment and strabismus for children younger than five years. Additional manoeuvres recommended for children include assessing for a red reflex and inspecting the external eye beginning with newborns. ${ }^{599}$

\section{Screening tests for visual impairment in adults}

For adults, both the Canadian Task Force on Preventive Health Care ${ }^{600}$ and the US Preventive Services Task Force ${ }^{601}$ recommend screening tests for visual impairment by primary care practitioners but suggest limiting these tests to people older than 65 years of age. Since these influential guidelines were published more than a decade ago, experts have recognized that the epidemiology of "best corrected" visual acuity on which they are based does not address uncorrected refractive error, ${ }^{602}$ now recognized as the leading cause of vision loss worldwide. ${ }^{576}$

\section{Other conditions}

In the case of glaucoma and diabetic retinopathy, patients do not typically present with vision impairment until damage has become irreversible. The Canadian Task Force on Preventive Health Care and the US Preventive Services Task Force have presented compelling evidence supporting recommendations for referral of asymptomatic adults at high risk (those older than 65 years, blacks older than 40 years, those with a firstdegree relative who has glaucoma and myopia exceeding -6 diopters) for glaucoma testing. ${ }^{600,603}$ Similarly, the Canadian Diabetes Association clinical practice guidelines make a compelling case for periodic screening tests for diabetic retinopathy in people with diabetes. ${ }^{604}$

\section{Take-home messages}

- Loss of vision and undiagnosed sight-threatening eye diseases are more common among new immigrants and refugees than in the general population.

- New immigrants should be screened for vision loss within their first year in Canada and should be referred to an optometrist or ophthalmologist if their presenting vision (with habitual correction in place) is less than 6/12 (i.e., less than 20/40).

- Referral for assessment is also warranted in the presence of other risk factors for blinding eye disease, including diabetes, age older than 65 years (or age older than 40 years for those of black ethnicity), glaucoma in a first-degree relative and myopia exceeding -6 diopters.

- Regionally prominent "tropical" eye diseases, such as onchocerciasis (river blindness), active trachoma and xerophthalmia, have not been reported in immigrants or refugees to Canada.

- Asymptomatic forms of these diseases should resolve or stabilize away from endemic conditions after arrival in Canada.

For the complete evidence review for vision health in immigrant populations, see Appendix 17, available at www.cmaj.ca/lookup /suppl/doi:10.1503/cmaj.090313/-/DC1 


\section{Contraception}

Reproductive and sexual health care is central to improving the health of women and is a basic human right. Couples and individuals have the right to "decide ... the number, spacing and time of their children and to have the information and the means to do so." ${ }^{{ }_{605}}$ Worldwide, about 120 million women have an unmet need for contraception. ${ }^{606,607}$ As a consequence, many experience unintended pregnancies, which can be associated with such negative outcomes as abortion, failure to adopt healthy pregnancy recommendations and limitation of women's ability to achieve educational, employment and economic goals. ${ }^{606,608}$ We reviewed the evidence for the effectiveness of screening and counselling for unmet contraceptive needs for immigrant populations. The recommendations of the Canadian Collaboration for Immigrant and Refugee Health related to contraception are outlined in Box 20A.

\section{Methods}

We used the 14-step method developed by the Canadian Collaboration on Immigrant and Refugee Health ${ }^{16}$ (summarized in section 3 of this article, above). We considered the epidemiology of unmet needs for contraception in immigrant populations and defined clinical considerations and potential key clinical actions. We searched MEDLINE, Embase, CINAHL, POPLINE, the Cochrane Library and

\section{Box 20A: Recommendations from the Canadian Collaboration for Immigrant and Refugee Health: contraception}

Screen immigrant women of reproductive age for unmet contraceptive needs.

Provide culturally sensitive, patient-centred contraceptive counselling to decrease unintended pregnancy and promote patient satisfaction.

\section{Basis of recommendation}

\section{Balance of benefits and harms}

Contraceptive counselling led to improved patient satisfaction (number needed to treat [NNT] 3, 95\% confidence interval $[\mathrm{Cl}] 2-5$ ) and improved continuation rates (NNT 4, 95\% Cl 3-7). Evidence that in-depth counselling reduces unintended pregnancy rates shows some uncertainty (relative risk $0.47,95 \% \mathrm{Cl} 0.16-1.34$ ); however, the committee judged that contraceptive continuation rates are an acceptable surrogate for unintended pregnancy rates. There is a high prevalence of unmet need for contraception among immigrant and refugee women $(5 \%-40 \%)$. Harms were minimal. No data were available on couple or family discord.

\section{Quality of evidence \\ Moderate}

\section{Values and preferences}

The committee attributed more value to supporting informed choice to meet future family needs and the woman's personal needs (empowerment) and less value to concern about causing couple and family discord. other sources from Jan. 1, 1996, to Jan. 1, 2010. Detailed methods, search terms, case studies and clinical considerations, as well as the complete reference list, can be found in the evidence review for contraception (Appendix 18, available at www.cmaj.ca/lookup/suppl/doi:10.1503/cmaj.090313 /-/DC1).

\section{Results}

We found no systematic reviews or guidelines for immigrant screening related to contraception. Our search on contraceptive counselling in the general population yielded 789 titles. We retained four reviews, two guidelines and six primary studies as the basis for our evidence..$^{64,609-619}$ In our search to update the reference systematic reviews, we found 203 new studies. Our evidence comes from studies in both general and high-risk populations of sexually active women from developed, low-income and middle-income countries.

\section{What unmet contraceptive needs affect immigrant and refugee women?}

Most immigrants to Canada come from developing countries, where unmet needs for contraception range from 5\% to $40 \%$. Rates are highest in sub-Saharan Africa, among young women and women who have had more than three births. ${ }^{608}$ Rural, uneducated and poor women are also at higher risk. ${ }^{608,620}$ Worldwide, more than one-third of pregnancies are unintended, and $50 \%$ of these end in abortion. ${ }^{606}$

Studies from Europe suggest that immigrant and refugee women have higher rates of unintended pregnancy and abortion than native-born women, and more than half of immigrants who seek abortion are not using any form of contraception. ${ }^{621}$ In a recent US study, ${ }^{622}$ foreign-born and native-born women had similar abortion rates, although rates were higher for visible minority and poorer women, characteristics that are associated with immigrant status in Canada. Immigrant and refugee women are also less likely than the general population to seek counselling for family planning (Table 20A). ${ }^{618,619}$

\section{Do screening and counselling decrease unintended pregnancy or increase patient satisfaction?}

A Guatemalan study provided moderate-quality evidence that screening and counselling are associated with an increase in informed contraceptive choice (relative risk [RR] 3.25, 95\% confidence interval [CI] 1.55-6.82) and receipt of or referral for a contraceptive method (RR 2.65, 95\% CI 1.48-4.74). ${ }^{618}$ One high-quality randomized trial found that women using medroxyprogesterone who received structured counselling about side effects were more likely to continue the method 
than those who received regular counselling (Table 20B). ${ }^{614} \mathrm{In}$ another randomized trial, which provided moderate-quality evidence for individualized counselling and follow-up, women attending a sexually transmitted disease clinic who had individualized contraceptive counselling and follow-up had higher rates of effective contraceptive use at four and eight months than those receiving regular information on options, although the effect diminished over time and by 12 months was not statistically significant. ${ }^{615}$ The cohort study by Nawar and colleagues ${ }^{617}$ of 590 Egyptian women provided moderate-quality evidence that women receiving clientcentred care are more satisfied than those receiving usual care (RR 2.17, 95\% CI 1.76-2.68). We found no data on harm of screening or contraceptive counselling, other than personnel and costs.

Evidence from systematic reviews, observational studies and guidelines suggests that a client-centred approach, giving women their method of choice, providing the contraceptive method on site and having a good personal relationship, improve patient satisfaction and contraception continuation rates. ${ }^{609-611,623}$ Provider pressure to adopt a method has been shown to be associated with method discontinuation. A randomized trial demonstrated better knowledge improvement when a simpler rather than more detailed chart of contraceptive effectiveness was used. ${ }^{62}$ High-quality contraceptive care respects each woman's human and reproductive rights and enables her to make an informed contraceptive choice consistent with her personal values, needs and beliefs. ${ }^{62,526}$ (Tables $20 \mathrm{~A}$ and $20 \mathrm{~B}$ ).

\section{Clinical considerations}

\section{Which women might need special consideration for contraceptive counselling?}

With migration to a more stable environment, fertility rates of refugee women sometimes increase as they choose to rebuild families. Refugee women who have been in their country of resettlement for less than three months appear to have the highest levels of fertility of all resettled populations, although whether these pregnancies are intended or unintended is unknown. ${ }^{627}$ Alternatively, a desire to prevent pregnancy could increase if women perceive uncertainty and instability with migration. ${ }^{627,628}$ Contraceptive needs can fluctuate because of family reunification and an ability to visit partners in their home countries. Pregnancy intention, contraceptive options and emergency contraception should therefore be discussed early in resettlement and should be reassessed as circumstances change.

Unmarried women could be vulnerable to unintended pregnancy if cultural proscriptions on premarital sex prevent them from identifying and seeking support for their contraceptive needs. The most common reason for not using contraception among sexually active, unmarried women surveyed across all developing regions was a low perceived risk of pregnancy because of infrequent sexual activity. ${ }^{608}$

Adolescent risk-taking and experimentation with sexuality put teenagers at risk for unintended pregnancy. Most young people become sexually active between 15 and 19 years of age. In Canada, teenagers who are recent immigrants have lower rates of sexual activity and pregnancy than Canadian-

Table 20A: Summary of findings for contraceptive screening and counselling of women of reproductive age with unmet contraceptive needs

Patient or population: Women of reproductive age

Setting: Community health posts and clinics in Guatemala and Bolivia*

Intervention: Contraceptive screening and counselling

Comparison: Usual care

Sources: Mendez F, Lopez F, Brambila C, et al. Screening family planning needs: an operations research project in Guatemala. BMC Int Health Hum Rights 2004;4:2. ${ }^{618}$ Foreit JR, Vernon R, Hamel PR. Use of systematic screening to increase the provision of reproductive health services in Bolivia. FRONTIERS Final Report. Washington (DC): Population Council; $2005 .{ }^{619}$

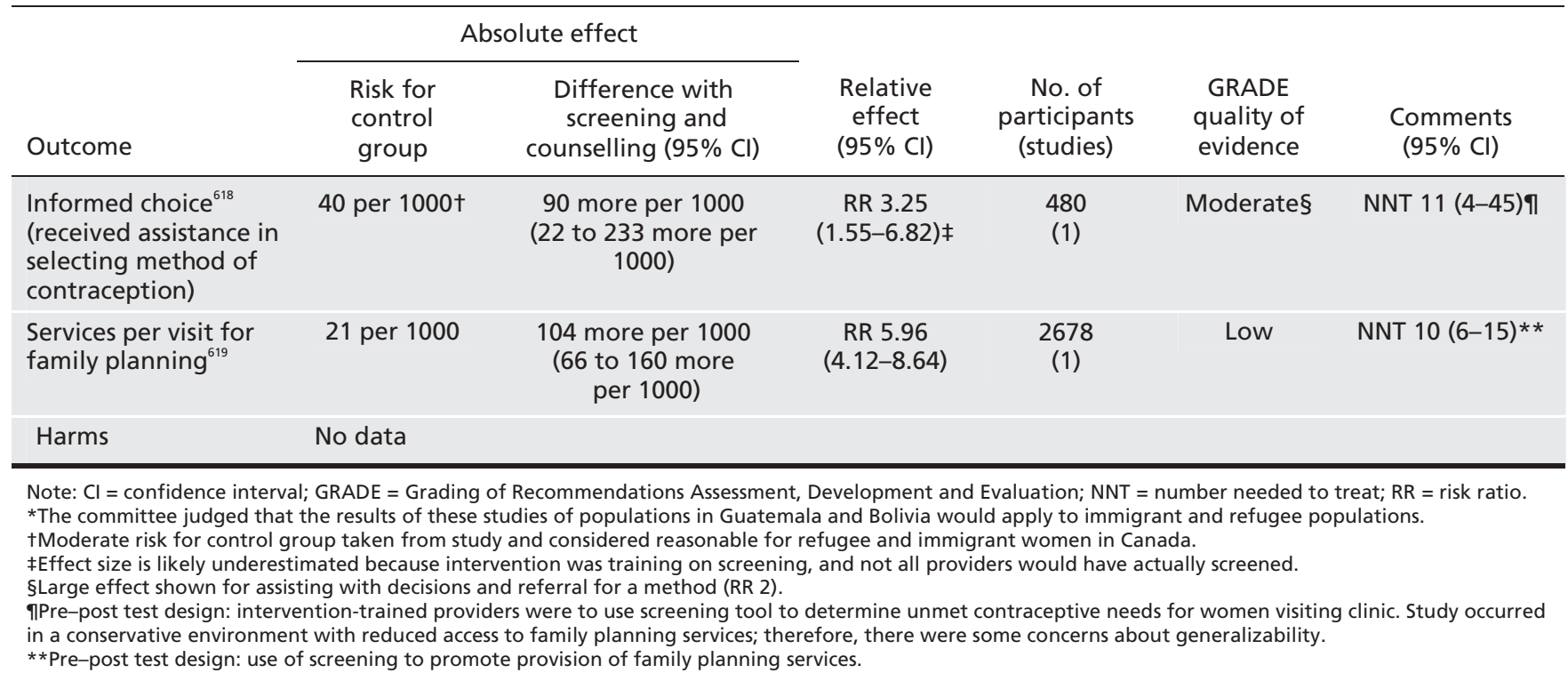


born teenagers. However, other countries of resettlement show the opposite pattern. Like North American-born teenagers, those from the developing world are interested in discussing sexual health concerns with health care providers, although needing to raise the topic, confidentiality concerns and parental presence often discourage this discussion. Adolescent newcomers sometimes experience conflict between their families' attitudes toward teenagers' sexuality and attitudes in their country of resettlement.

\section{What social and cultural factors influence contraceptive counselling?}

Women arriving from developing countries might have insufficient knowledge about reproduction and contraception to make an informed decision about family planning. ${ }^{608,620}$ Increased education and knowledge about reproduction correlate with more positive attitudes and increased use of and adherence to contraception. ${ }^{609}$ Women of all cultures use social networks for much of their sexual health information, which might be inaccurate or incomplete. ${ }^{620}$

Cultural attitudes toward pregnancy and family planning vary. Providential (“children are God's will") or pronatalist cultures discourage pregnancy prevention. In some cultures, women who bear many children are highly esteemed. ${ }^{628}$ Religious beliefs about the acceptability of contraceptive practices also influence some women. Contraception used to space births is acceptable in most religions. Among women of any particular faith, attitudes toward contraception vary widely. Health care providers should avoid assumptions and should assess each woman or couple individually ${ }^{608}$ Longer residence in the host country, educational and professional attainment, and youth favour positive attitudes toward modern contraception.

A woman might not perceive herself to be the decisionmaker for contraception, but she could be strongly influenced by her spouse, mother-in-law, sex role and religious beliefs. Worldwide, $11 \%-12 \%$ of married women do not use contra-

Table 20B: Summary of findings for contraceptive counselling of women of reproductive age with unmet contraceptive needs

Patient or population: Women of reproductive age with unmet contraceptive needs, ${ }^{614,615,617}$

Settings: Family planning clinics, ${ }^{614}$ primary health clinics ${ }^{617}$ and sexually transmitted diseases clinic ${ }^{615}$ in Mexico, Egypt and the United States Intervention: Contraceptive counselling

Comparison: Usual care

Sources: Canto De Cetina TE, Canto P, Ordoñez Luna M. Effect of counseling to improve compliance in Mexican women receiving depotmedroxyprogesterone acetate. Contraception 2001;63:143-6. ${ }^{614}$ Shlay JC, Mayhugh B, Foster M, et al. Initiating contraception in sexually transmitted disease clinic setting: a randomized trial. Am J Obstet Gynecol 2003; 189:473-81. ${ }^{615}$ Nawar L, Kharboush I, Ibrahim MA, et al. Impact of improved client-provider interaction on women's achievement of fertility goals in Egypt. FRONTIERS Final Report. Washington (DC): Population Council; $2004 .^{617}$

\begin{tabular}{|c|c|c|c|c|c|c|}
\hline \multirow[b]{2}{*}{ Outcome } & \multicolumn{2}{|c|}{ Absolute effect } & \multirow[b]{2}{*}{$\begin{array}{l}\text { Relative } \\
\text { effect } \\
(95 \% \mathrm{Cl})\end{array}$} & \multirow[b]{2}{*}{$\begin{array}{l}\text { No. of } \\
\text { participants } \\
\text { (studies) }\end{array}$} & \multirow[b]{2}{*}{$\begin{array}{l}\text { GRADE } \\
\text { quality of } \\
\text { evidence }\end{array}$} & \multirow[b]{2}{*}{$\begin{array}{c}\text { Comments } \\
(95 \% \mathrm{Cl})\end{array}$} \\
\hline & $\begin{array}{c}\text { Risk for } \\
\text { control group }\end{array}$ & $\begin{array}{c}\text { Difference with } \\
\text { contraceptive } \\
\text { counselling }(95 \% \mathrm{Cl})\end{array}$ & & & & \\
\hline \multirow{2}{*}{$\begin{array}{l}\text { Continuation }{ }^{614} \\
\text { (mean follow-up } \\
12 \text { mo) }\end{array}$} & \multicolumn{2}{|c|}{ Medium-risk population* } & \multirow{2}{*}{$\begin{array}{c}\text { RR } 1.46 \\
(1.27-1.7)\end{array}$} & \multirow{2}{*}{$\begin{array}{l}350 \\
(1)\end{array}$} & \multirow[t]{2}{*}{ High } & \multirow[t]{2}{*}{ NNT $4(3-7)$} \\
\hline & 560 per 1000 & $\begin{array}{c}258 \text { more per } 1000 \\
(151 \text { to } 392 \text { more } \\
\text { per } 1000)\end{array}$ & & & & \\
\hline \multirow{2}{*}{$\begin{array}{l}\text { Effective contraceptive } \\
\text { use }^{615} \text { (self-reported use of } \\
\text { effective method for }>75 \% \\
\text { of coitus or sexual } \\
\text { abstinence; mean } \\
\text { follow-up } 12 \text { mo) }\end{array}$} & \multicolumn{2}{|c|}{ High-risk population } & \multirow{2}{*}{$\begin{array}{c}\text { RR } 1.22 \\
(0.96-1.55)\end{array}$} & \multirow{2}{*}{$\begin{array}{c}632 \\
(1)\end{array}$} & \multirow[t]{2}{*}{ Moderate $†$} & \multirow{2}{*}{$\begin{array}{l}\text { NNT not } \\
\text { statistically } \\
\text { significant }\end{array}$} \\
\hline & 260 per 1000 & $\begin{array}{c}57 \text { more per } 1000 \\
\text { (10 fewer to } 143 \text { more } \\
\text { per } 1000)\end{array}$ & & & & \\
\hline \multirow{2}{*}{$\begin{array}{l}\text { Satisfaction }{ }^{617} \text { (no. of people } \\
\text { endorsing } 10-13 \text { of } 13 \text { items } \\
\text { related to satisfaction with } \\
\text { services; mean follow-up } 7 \\
\text { mo) }\end{array}$} & \multicolumn{2}{|c|}{ Medium-risk population } & \multirow{2}{*}{$\begin{array}{c}\text { RR } 2.17 \\
(1.76-2.68)\end{array}$} & \multirow{2}{*}{$\begin{array}{l}590 \\
(1)\end{array}$} & \multirow[t]{2}{*}{ Low } & \multirow[t]{2}{*}{ NNT $3(2-5)$} \\
\hline & 270 per 1000 & $\begin{array}{c}316 \text { more per } 1000 \\
(205 \text { to } 454 \text { more } \\
\text { per } 1000)\end{array}$ & & & & \\
\hline \multirow{4}{*}{$\begin{array}{l}\text { Unintended pregnancy }{ }^{615} \\
\text { (self-reported; mean } \\
\text { follow-up } 12 \text { mo) }\end{array}$} & \multicolumn{2}{|c|}{ Low-risk population } & \multirow{4}{*}{$\begin{array}{c}\text { RR } 0.83 \\
(0.63-1.09)\end{array}$} & \multirow{4}{*}{$\begin{array}{l}632 \\
(1)\end{array}$} & \multirow[t]{4}{*}{ Low } & \multirow{4}{*}{$\begin{array}{l}\text { NNT not } \\
\text { statistically } \\
\text { significant }\end{array}$} \\
\hline & 30 per 1000 & $\begin{array}{c}5 \text { fewer per } 1000 \\
(11 \text { fewer to } 3 \text { more } \\
\text { per 1000) }\end{array}$ & & & & \\
\hline & \multicolumn{2}{|c|}{ Medium-risk population } & & & & \\
\hline & 104 per 1000 & $\begin{array}{l}18 \text { fewer per } 1000 \\
\text { (38 fewer to } 9 \text { more } \\
\text { per } 1000 \text { ) }\end{array}$ & & & & \\
\hline
\end{tabular}

Note: $\mathrm{Cl}=$ confidence interval; GRADE = Grading of Recommendations Assessment, Development and Evaluation; NNT = number needed to treat; RR = risk ratio.

*Control-group risk of $56 \%$ continuation for medroxyprogesterone at 1 year from US national survey of family growth.

tSerious limitations because $30 \%$ lost to follow-up, self-reported unintended pregnancy rate, only $45 \%$ of women invited agreed to participate, women who declined were older, and method of randomization and blinding was not described.

fUnintended pregnancy data were rated as imprecise because RR was 0.47 (95\% Cl 0.00-1.34). 
ception because of opposition from one or more influential parties. In sub-Saharan Africa, this figure is $23 \%{ }^{608}$ Recognition of the partner's influence and his involvement, where appropriate, are important in counselling and supporting women's choices. Some men consider contraception to be their spouse's responsibility, but often this responsibility is shared, and involvement in counselling may be welcomed. In a population-based study of six African countries, ${ }^{629}$ women with supportive male partners were more likely to use modern contraceptives. In some traditional cultures, fathering many children is a sign of masculinity; however, perceived economic advantages of smaller families and better future opportunities for children can encourage men's support for contraception.

\section{How acceptable are specific contraceptive methods?}

Contraceptive use is increasing worldwide, but the mix and acceptability of contraceptive methods vary (Table 20C). ${ }^{626}$ The average rate of contraceptive use by married women from low- and middle-income countries is $60 \%$, with highest rates in Latin America and the Caribbean and much lower rates in sub-Saharan Africa. ${ }^{626}$ Effectiveness and freedom from adverse effects are the most important characteristics influencing contraceptive choice. Reluctance to use modern methods (e.g., oral contraceptives) can be influenced by culturespecific fear of adverse effects. For example, although many North American women choose to eliminate menstrual bleeding, those from African cultures often prefer monthly bleeding. Spotting and bleeding associated with some methods are problems for women who have religious and cultural restrictions on intercourse or other activities related to bleeding. ${ }^{610}$

In many African and Latin American populations, condom use has connotations of infidelity, promiscuity, extramarital relationships or sexually transmitted infection. Although used by only $1 \%-2 \%$ of North American women aged $15-44$ years, the intrauterine device is the most commonly used and most effective reversible contraceptive method worldwide. ${ }^{626,630,631}$

In sub-Saharan Africa, about $25 \%$ of women practising contraception use injectables. Breastfeeding is commonly used in low- and middle-income countries to control fertility.

Worldwide, many couples depend on tubal ligation for contraception. Some religions prohibit sterilization, and in many countries, tubal ligation is illegal or spousal consent is required. Most women from developing countries are unaware of emergency contraception. Availability of legal abortion varies greatly around the world, as does its cultural acceptance. Abortion rates are highest in Eastern Europe and central Asia, where contraceptive options have been limited and abortions freely available..$^{632}$

\section{What are the medical considerations?}

Condom use should be encouraged for women at risk for sexually transmitted infections, irrespective of additional contraceptive use. More common in immigrant and refugee women, HIV infection and sickle cell anemia bear special consideration. Guidelines suggest that hormonal methods can be safely used by HIV-positive women. Although serum levels of contraceptive hormones can be reduced by some antiretroviral medications, the clinical implications are unknown. ${ }^{625}$ Intrauterine devices are considered safe for women with HIV who are immunocompetent. ${ }^{62}$ Pregnancy is risky for women with sickle cell anemia. Although combination hormonal contraceptives are considered reasonably safe for women with sickle cell anemia, ${ }^{625}$ progestin-only contraceptives such as medroxyprogesterone have the added benefit of reducing sickle cell crises. ${ }^{633,634}$

\section{What are the potential implementation issues?}

Language barriers and lack of familiarity with the Canadian

Table 20C: Regional prevalence of use of main contraceptive methods, from most effective (sterilization) to least effective (periodic abstinence $^{626}$

Contraceptive method; \% of women of reproductive age in a marital or other union

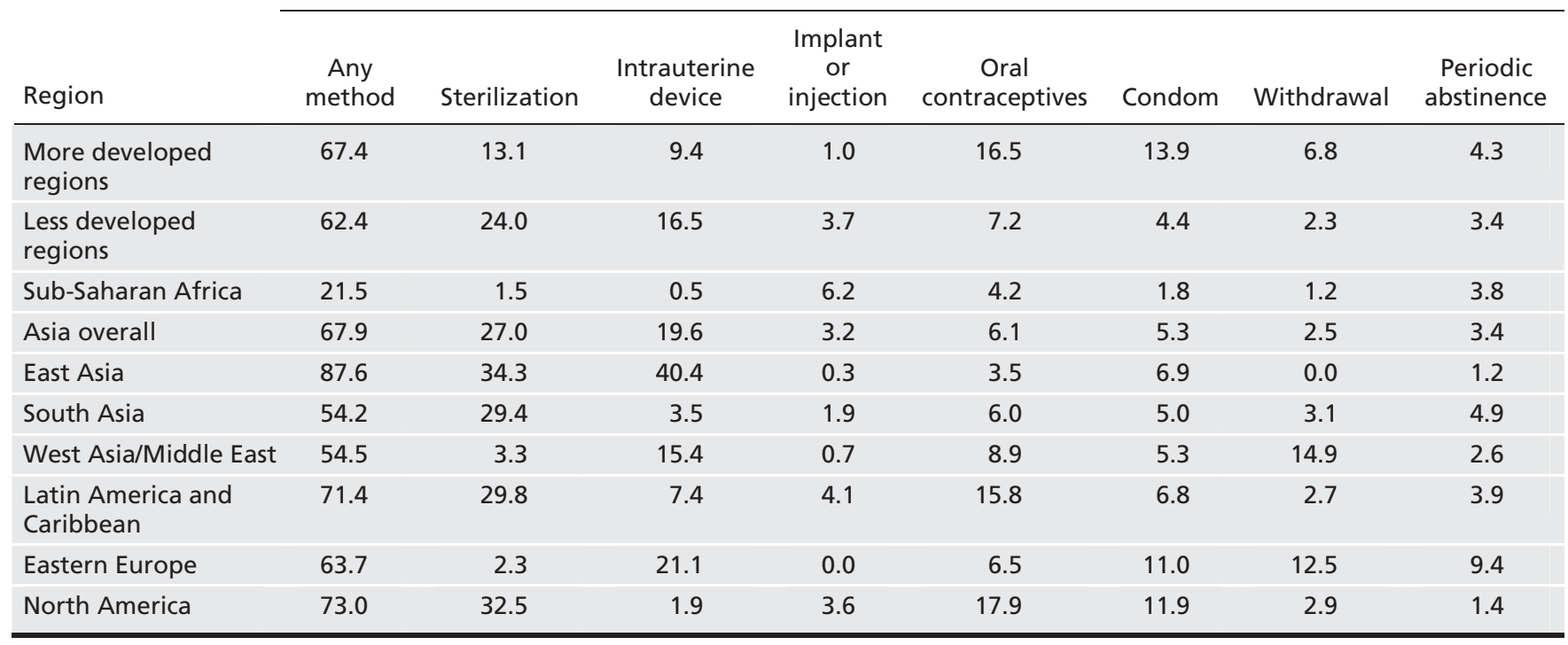


health care system can limit immigrant and refugee women's access to contraceptive care. In some communities of origin, the husband's accompaniment or written consent is required to obtain contraception. ${ }^{635}$ In others, hormonal contraception is available in pharmacies without a prescription.

The caregiver's sex is important for women in many cultures, particularly for refugee women, many of whom have a history of sexual assault and abuse. The advantages of ethnic and sex matching must be weighed against research suggesting a preference for "Canadian" professionals by immigrant and refugee women. ${ }^{636}$ Unnecessary medical barriers, such as examinations, blood tests and Papanicolaou smears, and a lack of culturally appropriate teaching aids are additional obstacles to contraceptive use. ${ }^{610}$

The Interim Federal Health Program covers the cost of contraceptives for Convention refugees, refugee claimants and protected people. Newcomers without health insurance can be guided to publicly funded sexual health clinics that provide services and low-cost contraceptives, regardless of health insurance status. The Society of Obstetricians and Gynaecologists of Canada also has a Compassionate Contraceptive Assistance Program that assists women in financial need (www .sogc.org/compassionate/pdf/compassionnate_form_e.pdf).

\section{Recommendations of other groups}

In 1996, the US Preventive Services Task Force recommended periodic counselling to prevent unintended pregnancy in teenagers and women of reproductive age, based on information taken from a sexual history ${ }^{612}$ However, subsequent guidelines from this task force do not include this recommendation. The Institute for Clinical Systems Improvement gave prevention counselling for unintended pregnancy a level III recommendation (incomplete evidence and action left to judgment of group or clinician). ${ }^{637}$ Our recommendations highlight the importance of early screening of immigrant women for unmet contraceptive needs.

\section{Take-home messages}

- Screening for unmet contraceptive needs among immigrant women should begin soon after their arrival in Canada.

- Women from developing countries are often unaware of emergency contraception.

- Acceptability of contraception and preferences for particular methods vary across world regions (e.g., use of intrauterine devices is predominant in Asia and Latin America).

- In some communities, condoms have connotations of infidelity, promiscuity or sexually transmitted infection or are used only with nonmarital partners.

- Giving women their method of choice, providing the contraceptive method on site and having a good personal relationship improve outcomes.

For the complete evidence review for contraception in immigrant populations, see Appendix 18, available at www.cmaj.ca/lookup /suppl/doi:10.1503/cmaj.090313/-/DC1 


\section{Cervical cancer}

Cervical cancer is one of the most preventable forms of cancer, yet deaths from cervical cancer persist among socially disadvantaged groups. ${ }^{638,639}$ The introduction of cervical cancer screening programs is associated with dramatic decreases in morbidity and mortality from cervical cancer. ${ }^{640,641}$ However, Canadian studies have documented significantly lower rates of screening among immigrants and refugees. ${ }^{6,642}$ Recent data from the Canadian Human Mortality Database (2000-2002) ${ }^{643}$ suggest a high mortality rate from cervical cancer among foreign-born women. We reviewed the evidence related to cervical cancer to guide practitioners in the prevention, early detection and treatment of cervical cancer for new immigrants. The recommendations of the Canadian Collaboration for Immigrant and Refugee Health on prevention of and screening for cervical cancer are outlined in Box 21A.

\section{Methods}

We used the 14-step method developed by the Canadian Collaboration for Immigrant and Refugee Health ${ }^{16}$ (summarized in section 3 of this article, above). We considered the epidemiology of cervical cancer in immigrant populations and defined clinical preventive actions (interventions), outcomes and key clinical questions. We searched MEDLINE, Embase, CINAHL, the Cochrane Library and other sources from Jan. 1, 1996, to Jan. 1, 2010. Detailed methods, search terms, case studies and clinical considerations can be found in the complete evidence review for cervical cancer (Appendix 19, available at www.cmaj.ca/lookup/suppl/doi:10.1503/cmaj.090313 /-/DC1).

\section{Results}

We found no systematic reviews or guidelines specifically focused on immigrants. We identified 13 (out of 934) general population titles as relevant systematic reviews and, after critical appraisal, retained eight. ${ }^{64-651}$ Our search for new and pertinent studies identified four cohort studies relating to cervical cytology screening..$^{652-655}$ A related study ${ }^{600}$ provided historical time trend mortality data related to cervical cancer screening programs. Finally, we identified one additional study reporting on the adverse events from human papillomavirus (HPV) vaccination in Australia ${ }^{656}$ and one meta-analysis reporting adverse pregnancy outcomes associated with treatment of cervical dysplasia. ${ }^{67}$ We retrieved 104 articles that addressed epidemiology, knowledge and compliance, and vaccination in immigrant populations.

\section{What is the burden of cervical cancer in immigrant populations?}

Data from the Canadian Human Mortality Database ${ }^{643}$ (nonage-standardized) showed that mortality rates from cervical cancer were 1.4 times higher among foreign-born women than among Canadian-born women (2000-2002). In the United States, the incidence of cervical cancer among VietnameseAmerican women has been estimated at five times the incidence among white American women (incidence rate 43 per 100000 v. 8.7 per 100 000). ${ }^{657}$ The Public Health Agency of Canada linked a sample of immigrants arriving to Canada (1980-1990) with cancer incidence data for the period (1980 1998) and found that overall in this cohort, foreign-born women had lower incidence rates of cervical cancer than Canadian-born women. However, rates among refugee women and Canadian-born women were similar, and older refugee women had higher rates of cervical cancer than Canadian-born women. ${ }^{658}$ Women who have never had cervical screening, or have not had cervical screening in the previous five years, account for $60 \%-90 \%$ of invasive cervical cancers overall. ${ }^{650}$ Also, several cross-sectional Canadian studies have

\section{Box 21A: Recommendations from the Canadian Collaboration for Immigrant and Refugee Health: cervical cancer}

Vaccination against human papillomavirus (HPV)

Vaccinate 9- to 26-year-old female patients against HPV.

Basis of recommendation

\section{Balance of benefits and harms}

Vaccination against HPV prevented invasive changes related to cervical cancer (number needed to vaccinate $139,95 \%$ confidence interval [CI] 117-180) in studies with a duration of 15-48 months. Access to cytology screening is often limited among immigrant women, and prevalence of HPV infection is higher in developing countries. Potential harms include anaphylaxis, which is amenable to treatment and which occurs in fewer than one in 100000 doses.

\section{Quality of evidence}

Moderate

Values and preferences

The committee attributed more value to preventing cervical cancer and less value to current uncertainty of long-term effect on cancer deaths.

\section{Cervical cytology screening}

Screen sexually active women for cervical abnormalities (by Papanicolaou test) to detect and treat invasive changes.

\section{Basis of recommendation}

\section{Balance of benefits and harms}

Identifying and treating early cervical cancer reduces mortality. The number needed to screen to prevent one death from cervical cancer is 3497 ( $95 \% \mathrm{Cl} 2361-90$ 909). The rate of cytology screening is lower among immigrant women than among Canadian-born women (40\%-60\% v. $60 \%-80 \%$ ). Harms are minimal and depend on the course of treatment.

\section{Quality of evidence}

Low

\section{Values and preferences}

The committee attributed more value to preventing cervical cancer and less value to uncertainty of size of effect and burden of screening on health services. 
documented lower rates of screening among immigrant populations. ${ }^{66,642}$ Foreign-born women aged 25-64 years, especially those born in Asia, are at higher risk of having never had a Papanicolaou (Pap) test (odds ratio 10.8). ${ }^{66}$

Infection with HPV is strongly associated with cervical cancer. Prevalence estimates for HPV are particularly high for Africa $(22.1 \%$, 95\% confidence interval [CI] 20.9\%$23.4 \%)$ and Central America $(20.4 \%$, $95 \%$ CI $19.3 \%-$ 21.4\%). ${ }^{659,660}$ Women with HIV and women who have been victims of sexual trauma are at higher risk for HPV infection and cervical cancer ${ }^{646}$ Refugee women in particular are disproportionately victims of sexual and sex-based violence, which can include rape, domestic violence and female genital mutilation.

\section{Does vaccination against HPV decrease morbidity and mortality?}

\section{Relative benefits and harms of vaccination}

A systematic review of vaccination against HPV showed reduction of high-grade cervical cancer lesions with no serious adverse events ${ }^{647}$ (Table 21A). We downgraded the quality of this evidence to moderate because of indirectness, since high-grade cervical lesions are considered surrogate outcomes for cervical cancer mortality. There were fewer than 15 anaphylactic events in a longitudinal study of more than one million doses of HPV vaccination among women in Aus- tralia. ${ }^{656}$ This adverse reaction occurred within 15 minutes of vaccination and was amenable to treatment.

\section{Does cervical cancer cytology screening and treatment decrease morbidity and mortality?}

\section{Screening tests}

Cervical cytology testing (liquid-based or conventional) is $60 \%-80 \%$ sensitive for high-grade lesions and $98 \%$ specific. ${ }^{645}$ Identifying cervical cancer using testing for HPV DNA is more sensitive but less specific than cervical cytology. ${ }^{661}$ Ninety-two per cent of women will survive five years when cervical cancer is localized, but only $13 \%$ will survive distant disease. ${ }^{644}$

\section{Relative benefits and harms of screening and treatment programs}

For cervical cancer screening programs, we found two largescale observational studies ${ }^{653,654}$ and two systematic reviews. ${ }^{644,645}$ The screening programs used invitation and reminder letters and practitioner incentives to increase screening rates from $61 \%$ to $83 \%$ of the population. Data from Rieck and coworkers ${ }^{654}$ showed a reduction in cervical cancer (relative risk $0.48,95 \%$ CI $0.23-0.98$ ) (Table 21B). Adverse effects resulting from referral for colposcopy include anxiety about pain and discomfort. ${ }^{662}$ However, no data quantifying these adverse effects were found. Perinatal

Table 21A: Summary of findings for prophylactic HPV vaccination against cervical cancer in women

Patient or population: Women aged 15-25 yr not previously infected with HPV (diverse ethnic backgrounds)

Setting: Multinational, but primarily North America, Latin America, Asia Pacific and Europe

Intervention: HPV vaccination

Comparison: Placebo or "no HPV vaccination"

Source: Rambout L, Hopkins L, Hutton B, et al. Prophylactic vaccination against human papillomavirus infection and disease in women: a systematic review of randomized controlled trials. CMAJ 2007;177:469-79. ${ }^{647}$

\begin{tabular}{|c|c|c|c|c|c|c|}
\hline Outcome & \multicolumn{2}{|c|}{ Absolute effect } & $\begin{array}{l}\text { Relative effect } \\
\quad(95 \% \mathrm{Cl})\end{array}$ & $\begin{array}{l}\text { No. of } \\
\text { participants } \\
\text { (studies) }\end{array}$ & $\begin{array}{l}\text { GRADE } \\
\text { quality of } \\
\text { evidence }\end{array}$ & $\begin{array}{l}\text { Comments } \\
(95 \% \mathrm{Cl})\end{array}$ \\
\hline $\begin{array}{l}\text { High-grade cervical } \\
\text { lesion }\end{array}$ & 15 per 1000 & $\begin{array}{l}7 \text { fewer per } 1000 \\
(8.5 \text { to } 5.5 \text { fewer } \\
\text { per } 1000)\end{array}$ & $\begin{array}{c}\text { RR } 0.52 \\
(0.43-0.63)\end{array}$ & $\begin{array}{c}36266 \\
(5)\end{array}$ & Moderate & $\begin{array}{l}\text { NNT } 139 \\
(117-180)\end{array}$ \\
\hline $\begin{array}{l}\geq 1 \text { serious adverse } \\
\text { event* }\end{array}$ & 22 per 1000 & $\begin{array}{c}0 \text { more per } 1000 \\
(2 \text { fewer to } 4 \text { more } \\
\text { per } 1000)\end{array}$ & $\begin{array}{c}\text { RR } 1.00 \\
(0.87-1.14)\end{array}$ & $\begin{array}{c}39609 \\
(6)\end{array}$ & Moderate & $\begin{array}{l}\text { Two trials did not } \\
\text { report allocation } \\
\text { concealment }\end{array}$ \\
\hline $\begin{array}{l}\text { Death from adverse } \\
\text { event }\end{array}$ & $\begin{array}{l}60 \text { per } \\
100000\end{array}$ & $\begin{array}{c}6 \text { fewer per } 100000 \\
(37 \text { fewer to } 70 \\
\text { more per } 100000)\end{array}$ & $\begin{array}{c}\text { RR } 0.91 \\
(0.39-2.14)\end{array}$ & $\begin{array}{c}36783 \\
(4)\end{array}$ & Moderate & $\begin{array}{l}\text { Two trials did not } \\
\text { report allocation } \\
\text { concealment }\end{array}$ \\
\hline
\end{tabular}

Note: $\mathrm{Cl}=$ confidence interval; GRADE = Grading of Recommendations Assessment, Development and Evaluation; HPV = human papillomavirus; NA = not applicable; NNT = number needed to treat; $R R=$ relative risk.

*Serious adverse events: bronchospasm, gastroenteritis, headache, hypertension, pain at injection site or impaired joint movement in injected limb. 
mortality and adverse pregnancy outcomes have also emerged as rare though important potential harms in treatment of cervical intraepithelial neoplasm relevant for young women; however, the quality of this evidence remains very low $^{651}$ (Table 21B).

Introduction of screening programs to populations naive to screening reduces cervical cancer rates by $60 \%-90 \%$ within three years of implementation. ${ }^{663}$ Key factors that improve the effectiveness of programs include high participation rate, quality control in smear interpretation, reliable follow-up for abnormal results and facilities for adequate treatment. ${ }^{664}$

\section{Clinical considerations}

Initiating a pelvic examination with simple nonmedical language can build rapport, increase comfort and empower patients. ${ }^{665}$ Cervical cancer screening will be a new concept for many immigrant women. Factors that can reduce rates of screening among immigrant women included limited English- or French-language proficiency, employment and educational demands, transportation and childcare difficulties, as well as male physicians trying to respect patient's modesty. ${ }^{666}$ Before screening for cervical cancer in women who have been victims of sexual violence, practitioners should develop rapport, a process that can take several visits. Abuse may continue or increase after arrival in Canada because of additional stresses, so it is important to take time to listen to patients.

Women who perceive their risk of HPV infection as high are more accepting of HPV vaccination. ${ }^{677}$ Other factors influencing acceptance of HPV vaccine include perceived efficacy of the vaccine and physicians' recommendation of vaccination. The most common barrier to vaccine acceptance is cost. Evidence demonstrating the acceptability of vaccination to those at highest risk for cervical cancer (including ethnic minorities) is limited. ${ }^{67}$ In most provinces and territories in Canada, the HPV vaccine is publicly funded only for girls through a school-based immunization program, with no catch-up vaccination provision for newly arriving older immigrant girls.

\section{What are the potential implementation issues?}

Immigrant women often have little knowledge and many misconceptions about the benefits of screening for cervical cancer. Many cultures are very private regarding sexuality, leaving women reluctant to ask for cervical screening, and some women feel uncomfortable undressing in front of a stranger. ${ }^{668}$ Patients, especially female Muslim patients, may prefer female practitioners, ${ }^{668}$ and some also prefer a caregiver from the same culture. Immigrant community health workers and other community interventions that provide information and offer transport, female physicians and interpreters in informal clinic settings may improve uptake of screening..$^{65}$

\section{Recommendations of other groups}

The Canadian Immunization Committee recommends vaccination of Canadian female patients against HPV types 16 and 18. ${ }^{699}$ The Society of Obstetricians and Gynaecologists of Canada $^{670}$ and the Canadian Task Force on the Periodic Health Examination (now the Canadian Task Force on Preventive

Table 21B: Summary of findings for organized screening program compared with opportunistic screening to prevent cervical cancer

Patient or population: Sexually active women, target group for preventing cervical cancer

Setting: UK primary care ${ }^{653}$; Wales primary care ${ }^{654}$

Intervention: Organized screening program (invitation letters, practice-based incentives to improve screening rates)

Comparison: Opportunistic screening (screening recommended by practitioner without additional program aids)

Sources: Quinn M, Babb P, Jones J, et al. Effect of screening on incidence of and mortality from cancer of cervix in England: evaluation based on routinely collected statistics. BMJ 1999;318:904-8. ${ }^{653}$ Rieck GC, Tristram A, Hauke A, et al. Cervical screening in 20-24 year olds. J Med Screen 2006;13:64-71. .54 $^{20}$

\begin{tabular}{|c|c|c|c|c|c|c|}
\hline \multirow[b]{2}{*}{ Outcome } & \multicolumn{2}{|c|}{$\begin{array}{c}\text { Absolute effect in } \\
\text { medium-risk population }\end{array}$} & \multirow[b]{2}{*}{$\begin{array}{l}\text { Relative } \\
\text { effect } \\
(95 \% \mathrm{Cl})\end{array}$} & \multirow[b]{2}{*}{$\begin{array}{c}\text { No. of } \\
\text { participant } \\
\text { s (studies) }\end{array}$} & \multirow[b]{2}{*}{$\begin{array}{l}\text { GRADE } \\
\text { quality of } \\
\text { evidence }\end{array}$} & \multirow[b]{2}{*}{ Comments } \\
\hline & $\begin{array}{c}\text { Before } \\
\text { implementing } \\
\text { cytology }\end{array}$ & $\begin{array}{l}\text { Difference with } \\
\text { screening + recall }\end{array}$ & & & & \\
\hline Cervical cancer rate ${ }^{654}$ & 11 per 100000 & $\begin{array}{c}5 \text { fewer per } 100000 \\
(8.5 \text { to } 0 \text { fewer } \\
\text { per } 100000)\end{array}$ & $\begin{array}{c}\text { RR } 0.48 \\
(0.23-0.98)\end{array}$ & $\begin{array}{l}400000 \\
\text { (1) }\end{array}$ & Low & $\begin{array}{c}\text { NNT } 17483 \\
(11806-454545)\end{array}$ \\
\hline $\begin{array}{l}\text { Invasive cervical } \\
\text { cancer }\end{array}$ & 15 per 100000 & $\begin{array}{c}5 \text { fewer per } 100000 \\
(11.5 \text { fewer to } 7 \\
\text { more per } 100000)\end{array}$ & $\begin{array}{c}\text { RR } 0.67 \\
(0.30-1.48)\end{array}$ & $\begin{array}{l}200000 \\
\text { (1) }\end{array}$ & Very low & $\begin{array}{l}\text { NNT not statistically } \\
\text { significant }\end{array}$ \\
\hline $\begin{array}{l}\text { Death from cervical } \\
\text { cancer (surveillance) }^{654}\end{array}$ & 4 per 100000 & $\begin{array}{c}3 \text { fewer per } 100000 \\
(3.6 \text { fewer to } 1.6 \\
\text { more per } 100000)\end{array}$ & $\begin{array}{c}\text { RR } 0.38 \\
(0.10-1.41)\end{array}$ & $\begin{array}{l}400000 \\
\text { (1) }\end{array}$ & Very low & $\begin{array}{l}\text { NNT not statistically } \\
\text { significant }\end{array}$ \\
\hline
\end{tabular}

Note: $\mathrm{Cl}=$ confidence interval; GRADE = Grading of Recommendations Assessment, Development and Evaluation; NNT = number needed to treat; RR = relative risk 
Health Care $)^{671}$ recommend cervical cytology screening programs for sexually active adolescents and women. The International Agency for Research on Cancer recommends cervical screening for women 25-65 years of age and suggests a threeyear screening interval be considered in countries with adequate resources. ${ }^{638}$ Our recommendations highlight the need to improve the delivery of cervical cancer screening for immigrant women and vaccination against HPV.

\section{Take-home messages}

- Vaccination against HPV is recommended for 9- to 26year-old female patients to reduce invasive changes related to cervical cancer.
- All sexually active women should be screened for cervical abnormalities (with Pap smear) to detect and treat invasive changes.

- Providing clear information about cervical screening, building rapport and offering access to a female practitioner improve acceptance of Pap tests.

- Immigrant girls may miss out on school vaccination programs, depending on their age upon arrival in Canada.

For the complete evidence review for cervical cancer in immigrant women, see Appendix 19, available at www.cmaj.ca/lookup /suppl/doi:10.1503/cmaj.090313/-/DC1. 


\section{Pregnancy}

Every migrant woman, regardless of her status, has the fundamental right to receive complete prenatal, birth and postnatal care with dignity. ${ }^{672}$ However, evidence shows that access to pregnancy care is inadequate ${ }^{673-675}$ From 1996 to 2001, 22\% of all births in Canada were to foreign-born women..$^{20}$ Some births to migrant women are the result of sexual abuse in the case of civil unrest, sexual abuse during their flight from civil unrest, or sexual persuasion or favours granted to those organizing their transit to receiving countries ${ }^{676}$ Higher rates of cesarean section among newly arrived women $(33.0 \%-35.8 \%)$ than among Canadian-born women (26\%) have been reported. ${ }^{67}$ Maternal mortality rates in the United Kingdom for black African women are 5.6 times higher than for white women; for black Caribbean women they are 3.7 times higher, and for Middle Eastern women they are 2.9 times higher. ${ }^{677}$ Upon investigation, these higher mortality rates were found to be related to poor overall health, including unrecognized medical conditions, practitioners' ignorance of female genital mutilation, cultural practices, attitudes of male partners, inadequate interpreter services and suboptimal care. In this section we review and compile existing evidence for pregnancy care of women who have arrived in Canada within the past five years. The recommendations of the Canadian Collaboration for Immigrant and Refugee Health on pregnancy care are outlined in Box 22A.

\section{Methods}

We used the 14-step approach developed by the Canadian Collaboration for Immigrant and Refugee Health ${ }^{16}$ (summarized in section 3 of this article, above). We searched MEDLINE, Embase, CINAHL, HealthSTAR, the Cochrane Library and other sources from Jan. 1, 1996, to Jan. 1, 2010. We limited the literature search to the most salient issues for newly arrived pregnant women, and searched as systematically as possible, by focusing on issues with evidence of disease burden affecting this population more than other pregnant women, as well as on suggestions that clinical responses for this population should differ from responses for other pregnant women and on issues for which a concurrent evidence review was not already being conducted. Detailed methods, search terms, case studies and clinical considerations can be found in the complete evidence review for pregnancy (see Appendix 20, available at www.cmaj.ca/cgi/lookup/doi /10.1503/cmaj.090313/-/DC1).

\section{Results}

The search for guidelines and systematic reviews on pregnancy specific to immigrants yielded 20 titles, of which none were systematic reviews. After we applied our exclusion criteria to the search for articles on pregnancy for the general population, psychosocial concerns were restricted to social isolation, violence was restricted to sexual abuse, and prenatal screening for blood disorders was restricted to hemoglobinopathy. Work exposure and female genital mutilation remained as factors to consider in their entirety. Only four of 251 articles fulfilled our inclusion criteria. A further three were subsequently excluded because they lacked specificity for the screening or treatment intervention under consideration. The Committee of Obstetrics of the American College of Obstetricians and Gynecologists published clinical guidelines in 2007 and the Society of Obstetricians and Gynaecologists of Canada published clinical guidelines in 2008, both focused on hemoglobinopathy.

\section{How do pregnancy-related issues affect immigrant populations?}

\section{Social isolation}

One Canadian report showed that, after giving birth, $14.7 \%$ of asylum seekers and $7.7 \%$ of refugee women live alone, whereas $2.7 \%$ of Canadian-born women live alone. ${ }^{67}$ Perceived lack of social support was reported among $15.4 \%$ of immigrants versus $7.2 \%$ of Canadian-born women. ${ }^{678}$ Lack of psychosocial resources for nonmigrants has been found to lead to small-for-gestational-age infants. ${ }^{679}$

\section{Sexual abuse}

Sexual abuse is common in armed conflict and internal strife. Abuse is sometimes used to dominate, sexual torture can be used as a method of interrogation, and refugee camp guards and male refugees sometimes regard unaccompanied women and girls as common sexual property. Violence and rape

\section{Box 22A: Recommendations from the Canadian Collaboration for Immigrant and Refugee Health: pregnancy}

Develop and study interventions to reduce social isolation, given the risk for maternal morbidity and small-forgestational-age infants.

Basis of recommendation

\section{Balance of benefits and harms}

Pregnant immigrant and refugee women face an elevated risk of social isolation (15\% v. $7.5 \%$ for Canadian-born women), which is associated with maternal morbidity and small-for-gestational-age infants. However, in the absence of evidence showing that social interventions work, such interventions could cause harm. Therefore, the committee recommends development and study of interventions for pregnant immigrant and refugee women who are socially isolated.

\section{Quality of evidence}

Very low, with no intervention evidence available Values and preferences

The committee attributed more value to preventing uncertain harms than to providing uncertain benefits through unstudied social interventions. 
among refugee women in camps ranges from $24.4 \%$ to $40.0 \%{ }^{680}$ Many refugee women (23\%-50\%) also report violence at the hands of their husbands. ${ }^{680}$ Pregnancy-related effects include unwanted pregnancies, sexually transmitted infections, chronic pelvic infection, reproductive tract trauma, psychological trauma, social rejection, and mistreatment or abandonment of resulting infants. ${ }^{676}$

\section{Hemoglobinopathy}

Hemoglobinopathy (predominantly thalassemia and sicklecell anemia) is more common among newly arrived women from certain regions of the world. ${ }^{681-683}$ Hemoglobinopathy can lead to serious maternal complications, severe anemia in infants and painful vaso-occlusive crises. ${ }^{682}$

\section{Exposure to hazards in the workplace}

Newly arrived women are often exposed to unprotected and unregulated work environments, where the risk of adverse pregnancy outcomes is great. Their lack of knowledge of employee rights exaggerates these exposures.

\section{Female genital mutilation}

The prevalence of female genital mutilation ranges from 5\% to $97 \%$ among women in countries where it is practised. ${ }^{684}$ There are four types of female genital mutilation: type I, excision of the prepuce, with or without excision of all or part of the clitoris; type II, excision of the clitoris with partial or total removal of the labia minora; type III, excision of part or all of the external genitalia and stitching or narrowing of the vaginal opening, also called "infibulation"; and type IV, "unclassified," which can include pricking, piercing, incising, stretching, cauterizing, scraping, cutting, introducing corrosive substances or other methods. Type III causes a mechanical barrier to delivery; however, types I, II and IV can produce severe vulval and vaginal scarring that may also obstruct delivery. ${ }^{685}$ Types II and III are associated with greater risks of cesarean section, postpartum hemorrhage, extended hospital stay, infant resuscitation, and stillbirth or early neonatal death. ${ }^{686}$ An estimated $15 \%$ of all circumcised women have undergone type III mutilation. ${ }^{67}$ Most cases of female genital mutilation in Djibouti, Somalia and Sudan (80\%-90\%) are type III. ${ }^{687}$ Practitioners attending Somalian births in Canada have been found to lack knowledge of female genital mutilation and to manifest unprofessional attitudes toward these women. ${ }^{688}$

\section{Does screening for social isolation or exposure to unregulated work environments decrease pregnancy-related morbidity and mortality?}

Currently, no screening tools or interventions, apart from provision of information (www.workrights.ca), focus specifically on social isolation or exposure to unprotected work environments among pregnant women. Although these topics are recognized as issues in the literature, no relevant guidelines or systematic reviews were specific to pregnant women.

\section{Clinical considerations}

Language barriers and cultural considerations ${ }^{689-691}$ as well as eligibility for health services, play important roles in access to care. Patients may fear that requests for additional services will reduce their chances of successfully remaining in Canada. Practitioners should consider the patient's interest in screening for and treating these conditions, ${ }^{683}$ eligibility for services ${ }^{692}$ and interacting in culturally sensitive ways about these issues. ${ }^{68.933-696}$ Treatment preferences and expectations are often based on what was done in the country of origin, which could differ from Canadian care (e.g., expectation of a greater number of ultrasound examinations). The procedures themselves could be unacceptable (e.g., amniocentesis) or the notion of statistical probability might not be well understood. Further, if the testing must be paid for and families have limited resources, they could be unable to have the tests done. Closely spaced pregnancies (resulting in physical health problems and difficult social integration) are another culturally driven consideration.

Primary care practitioners in the author group noted that the woman's health and pregnancy history might be incorrect or incomplete for several reasons. She could fear jeopardizing her asylum or claimant application (e.g., if the application states she has no other children, she might not want to report now that she has other children), her records from the previous country might be unavailable, or she could have delayed the start of prenatal care. These issues could be particularly important in determining the appropriateness of a cesarean birth.

In Canada, immigrants face a three-month waiting period for provincial health coverage in certain provinces, limited numbers of clinicians accept the Interim Federal Health Program plan, and migrants often lack of awareness of services. ${ }^{67,675}$ Delayed prenatal care in Ontario has been found to be as high as $60 \%$ in migrant pregnant women. ${ }^{697}$

\section{Recommendations of other groups}

The recommendations of other groups in three specific areas are outlined below. Our recommendations highlight the research needed to develop interventions and to reduce social isolation for pregnant immigrant women.

\section{Female genital mutilation}

Recommendations from the World Health Organization are available, ${ }^{685}$ as is a review of clinical practice. ${ }^{698}$ Guidelines for care in cases of female genital mutilation were published by Health Canada, ${ }^{699}$ although no evidence of the effectiveness of suggested interventions was provided. An article entitled "What Somali women say about giving birth in Canada" 688 provides suggestions for providing more respectful and less interventionist care, as well as greater sensitivity for crosscultural practices.

\section{Sexual abuse}

Health Canada's guidelines on family-centred maternity and newborn care (published in 2000) ${ }^{699}$ recommend using the ALPHA form ${ }^{700}$ to assess current and past abuse (including 
sexual abuse), although sensitivity and specificity (and other measures) are not provided for this tool. ${ }^{701}$ No recommendations are available specific to the screening and care of sexual abuse victims during pregnancy, a time during which bodily changes and vaginal examinations can elicit untoward memories of sexual abuses.

\section{Hemoglobinopathy}

The guidelines of the Society of Obstetricians and Gynaecologists of Canada ${ }^{62}$ recommend that those who originate from countries outside northern Europe should be considered at high risk for hemoglobinopathy. As well "the definitive test for carriers of hemoglobin S, C, or D is hemoglobin electrophoresis, which should be offered to all couples of African or Caribbean descent." ${ }_{682}$ The guidelines of the American College of Obstetricians and Gynecologists' (based on level A scientific evidence, i.e., good and consistent) ${ }^{702}$ note that "Individuals of African, Southeast Asian, and Mediterranean descent are at increased risk for being carriers of hemoglobinopathies and should be offered carrier screening and, if both parents are determined to be carriers, genetic counselling."

\section{Take-home messages}

- Women newly arrived to Canada are responsible for an important proportion of total births.

- In the hopes of reducing maternal morbidity and reducing the number of infants who are small for their gestational age, further development and study is required of interventions to address the social isolation of immigrants and refugees who are pregnant.

- However, we lack data to support actions to be taken by primary care practitioners related to these disparities.

For the complete evidence review for pregnancy in immigrant populations, see Appendix 20, available at www.cmaj.ca/lookup /suppl/doi:10.1503/cmaj.090313/-/DC1. 


\section{References}

1. International Organization for Migration. The world migration report 2008: managing labour mobility in the evolving global economy. Geneva $(\mathrm{CH})$ : The Organization; 2008 .

2. MacPherson DW, Gushulak BD, Macdonald L. Health and foreign policy: influences of migration and population mobility. Bull World Health Organ 2007;85: 200-6.

3. Human development report 2009. Overcoming barriers: human mobility and development. New York (NY): United Nations Development Program, Human Development Report Office; 2009. Available: http://hdr.undp.org/en/reports/global/hdr2009 /chapters/ (accessed 2010 May 25).

4. Projections of the diversity of the Canadian population 2006-2031. Ottawa (ON): Statistics Canada; 2010

5. Gushulak BD, Pottie K, Hatcher Roberts J, et al. Migration and health in Canada: health in the global village. CMAJ 2010 June 28 [Epub ahead of print].

6. Facts and figures 2007. Ottawa (ON): Citizenship and Immigration Canada; 2008. Cat. no. Ci1-8/2007E.PDF.

7. Reitz JG. Closing the gaps between skilled immigration and Canadian labour markets: emerging policy issues and priorities. Toronto $(\mathrm{ON})$ : University of Toronto; 2007.

8. Visiting Canada. Ottawa (ON): Citizenship and Immigration Canada; 2007. Available: www.cic.gc.ca/english/visit/index.asp (accessed 2010 Apr. 21).

9. Wilkins R, Tjepkema M, Mustar C, et al. The Canadian census mortality follow-up study, 1991 through 2001. Health Rep 2008;19:25-43

10. DesMeules M, Gold J, McDermott S, et al. Disparities in mortality patterns among Canadian immigrants and refugees, 1980-1998: results of a national cohort study. $J$ Immigr Health 2005;7:221-32.

11. Steele LS, Lemieux C, Clark J, et al. The impact of policy changes on the health of recent immigrants and refugees in the inner city. Can J Public Health 2002;93:118-22.

12. Commission on Social Determinants of Health. Closing the gap in a generation: health equity through action on the social determinants of health. Final report. Geneva $(\mathrm{CH})$ : World Health Organization; 2008. Available: www.who.int/social _determinants/en/ (accessed 2011 Feb. 10).

13. Beach MC, Gary T, Price E, et al. Improving health care quality for racial/ethnic minorities: a systematic review of the best evidence regarding provider and organization interventions. BMC Public Health 2006;6:104

14. Pottie K, Torres S. Systematic review for guidelines for immigrants and refugees: policy report for the Public Health Agency of Canada. Ottawa (ON): Canadian Collaboration for Immigrant and Refugee Health; 2005. Available: www.ccirh.uottawa .ca/eng/index.html (accessed 2011 Feb. 10)

15. Swinkels H, Pottie K, Tugwell P, et al. Development of guidelines for recently arrived immigrants and refugees to Canada: Delphi consensus on selecting preventable and treatable conditions. CMAJ 2010 June 28 [Epub ahead of print].

16. Tugwell P, Pottie K, Welch V, et al. Evaluation of evidence-based literature and formation of recommendations for the clinical preventive guidelines for immigrants and refugees in Canada. CMAJ 2010 June 23 [Epub ahead of print]

17. Schünemann HJ, Jaeschke R, Cook DJ, et al. An official ATS statement: grading the quality of evidence and strength of recommendations in ATS guidelines and recommendations. Am J Respir Crit Care Med 2006;174:605-14.

18. Guyatt GH, Oxman AD, Vist GE, et al. GRADE: an emerging consensus on rating quality of evidence and strength of recommendations. BMJ 2008;336:1049-51.

19. San Martin C, Ross N. Experiencing difficulties accessing first-contact health services in Canada. Healthc Policy 2006;1:103-19.

20. Gushulak BD, MacPherson DW. Migration medicine and health: principles and practice. Hamilton (ON): BC Decker; 2006.

21. Australian Government, National Health and Medical Research Council AG. Cultural competency in health: a guide for policy, partnerships and participation. Canberra (AU): The Council; 2005.

22. Walker PF, Barnett ED, Stauffer WM, editors. Immigrant medicine. Philadelphia (PA): Saunders Elsevier; 2007

23. Murray R, Davis J, Krause V, et al. Diagnosis, management and prevention of infections in recently arrived refugees. Sydney (AU): Australasian Society for Infectious Diseases; 2009. Available: www.asid.net.au/downloads/RefugeeGuidelines.pdf (accessed 2011 May 5).

24. Barnett ED. Infectious disease screening for refugees resettled in the United States. Clin Infect Dis 2004;39:833-41

25. Stauffer WM, Karnat D, Walker PF. Screening of international immigrants, refugees, and adoptees. Prim Care 2002;29:879-905.

26. Gavagan T, Brodyaga L. Medical care for immigrants and refugees. Am Fam Physician 1998;57:1061-8.

27. HPA migrant health guide. London (UK): Health Protection Agency; 2011. Available: www.hpa.org.uk/migranthealthguide (accessed 2011 Feb. 10)

28. Kirmayer LJ, Narasiah L, Munoz M, et al. Common mental health problems in immigrants and refugees: general approach in primary care. CMAJ 2010 July 5 [Epub ahead of print].

29. Immigration and Refugee Protection Act, S.C. 2001, c. 27

30. Hyman I. Immigration and health: reviewing evidence of the healthy immigrant effect in Canada. CERIS Working Paper No. 55. Toronto (ON): Joint Centre of Excellence for Research on Immigration and Settlement; 2007

31. Ng E, Wilkins R, Gendron F, et al. Dynamics of immigrants' health in Canada: evidence from the National Population Health Survey. Ottawa (ON): Statistics Canada; 2005

32. Newbold KB, Danforth J. Health status and Canada's immigrant population. Soc
Sci Med 2003;57:1981-95

33. Dunn JR, Dyck I. Social determinants of health in Canada's immigrant population: results from the National Population Health Survey. Soc Sci Med 2000;51:1573-93.

34. McDonald JT, Kennedy S. Insights into the 'healthy immigrant effect': health status and health service use of immigrants to Canada. Soc Sci Med 2004;59:1613-27.

35. Ng E, Pottie K, Spitzer D. Limited official language proficiency and decline in health status: a dynamic view from the longitudinal survey of immigrants to Canada. Health Rep. In press.

36. Pottie K, Ng E, Spitzer D, et al. Language proficiency, gender and self-reported health: an analysis of the first two waves of the Longitudinal Survey of Immigrants to Canada. Can J Public Health 2008;99:505-10.

37. Yuan L, Lau W, Thipphawong J, et al. Diphtheria and tetanus immunity among blood donors in Toronto. CMAJ 1997;156:985-90

38. Greenaway C, Dongier P, Boivin JF, et al. Susceptibility to measles, mumps, and rubella in newly arrived adult immigrants and refugees. Ann Intern Med 2007;146: 20-4.

39. Christiansen D, Barnett ED. Comparison of varicella history with presence of varicella antibody in refugees. Vaccine 2004;22:4233-7.

40. Greenaway C, Dongier P, Boivin JF, et al. Viral hepatitis in newly arrived immigrants and refugees. 56th Annual Meeting of American Society of Tropical Medicine and Hygiene; 2007 Nov. 4-8.

41. Public Health Agency of Canada. Tuberculosis in Canada 2006: pre-release Ottawa (ON): The Agency; 2007.

42. Greenaway C, Sandoe A, Vissandjee B, et al. Tuberculosis: evidence review for newly arriving immigrants and refugees. CMAJ 2010 July 15 [Epub ahead of print]

43. Hirsch-Moverman Y, Daftary A, Franks J, et al. Adherence to treatment for latent tuberculosis infection: systematic review of studies in the US and Canada. Int J Tuberc Lung Dis 2008;12:1235-54.

44. Cass AD, Talavera GA, Gresham LS, et al. Structured behavioral intervention to increase children's adherence to treatment for latent tuberculosis infection. Int $J$ Tuberc Lung Dis 2005;9:415-20.

45. Newman PA, Williams CC, Massaquoi N, et al. HIV prevention for black women structural barriers and opportunities. J Health Care Poor Underserved 2008;19: 829-41.

46. Pattullo V, Heathcote J, Wong D. Superior reponse to pegylated interferon and ribavirin in Asians with chronic hepatitis C. Hepatol Int 2010;4:723-31.

47. Chan MS. The global burden of intestinal nematode infections - fifty years on Parasitol Today 1997;13:438-43.

48. Biggs BA, Caruana S, Mihrshahi S, et al. Management of chronic strongyloidiasis in immigrants and refugees: Is serologic testing useful? Am J Trop Med Hyg 2009; 80:788-91.

49. World Health Organization. World malaria report. Geneva $(\mathrm{CH})$ : The Organization; 2008.

50. Kain KC, Harrington MA, Tennyson S, et al. Imported malaria: prospective analysis of problems in diagnosis and management. Clin Infect Dis 1998;27:142-9.

51. Fazel M, Wheeler J, Danesh J. Prevalence of serious mental disorder in 7000 refugees resettled in Western countries: a systematic review. Lancet 2005;365: 1309-14.

52. Bhui K, Warfa N, Edonya $\mathrm{P}$, et al. Cultural competence in mental health care: a review of model evaluations. BMC Health Serv Res 2007;7:15.

53. Copeland WE, Keeler G, Angold A, et al. Traumatic events and posttraumatic stress in childhood. Arch Gen Psychiatry 2007;64:577-84

54. Kessler RC, Sonnega A, Bromet E, al. Posttraumatic stress disorder in the National Comorbidity Survey. Arch Gen Psychiatry 1995;52:1048-60.

55. Lane WG, Rubin D, Monteith R, et al. Racial differences in the evaluation of pediatric fractures for physical abuse. JAMA 2002;288:1603-9.

56. Taylor J, Baldwin N, Spencer N. Predicting child abuse and neglect: ethical, theoretical and methodological challenges. J Clin Nurs 2008;17:1193-200.

57. Creatore MI, Moineddin R, Booth G, et al. Age- and sex-related prevalence of diabetes mellitus among immigrants to Ontario, Canada. CMAJ 2010;182:781-9.

58. Hawthorne K, Robles Y, Cannings-John R, et al. Culturally appropriate health education for type 2 diabetes mellitus in ethnic minority groups. Cochrane Database Syst Rev 2008;(3):CD006424.

59. World Health Organization. Worldwide prevalence of anaemia 1993-2005. Geneva $(\mathrm{CH})$ : The Organization; 2008. Available: http://whqlibdoc.who.int/publications /2008/9789241596657_eng.pdf (accessed 2008 Sept. 10).

60. Bindra GS, Gibson RS. Iron status of predominantly lacto-ovo vegetarian Eas Indian immigrants to Canada: a model approach. Am J Clin Nutr 1986;44:643-52.

61. Matthews DC, Sutherland S, Basrani B. Emergency management of acute apical abscesses in the permanent dentition: a systematic review of the literature. J Can Dent Assoc 2003:69:660.

62. Bader JD, Rozier RG, Lohr KN, et al. Physicians' roles in preventing dental caries in preschool children: a summary of the evidence for the U.S. Preventive Services Task Force. Am J Prev Med 2004;26:315-25.

63. Resnikoff S, Pascolini D, Etya'ale D, et al. Global data on visual impairment in the year 2002. Bull World Health Organ 2004;82:844-51.

64. Halpern V, Grimes DA, Lopez L, et al. Strategies to improve adherence and acceptability of hormonal methods for contraception. Cochrane Database Syst Rev 2006 (1):CD004317

65. Lam TK, McPhee SJ, Mock J, et al. Encouraging Vietnamese-American women to obtain Pap tests through lay health worker outreach and media education. $J$ Gen Intern Med 2003;18:516-24

66. Maxwell CJ, Bancej CM, Snider J, et al. Factors important in promoting cervica 
cancer screening among Canadian women: findings from the 1996-97 National Population Health Survey (NPHS). Can J Public Health 2001;92:127-33.

67. Gagnon AJ, Dougherty G, Platt RW, et al. Refugee and refugee-claimant women and infants post-birth: migration histories as a predictor of Canadian health system response to needs. Can J Public Health 2007;98:287-91

68. van Doorslaer E, Masseria C, Koolman X. Inequalities in access to medical care by income in developed countries. CMAJ 2006;174:177-83.

69. Schünemann HJ, Hill SR, Kakad M, et al. Transparent development of the WHO rapid advice guidelines. PLoS Med 2007;4:e119.

70. Oxman AD, Schünemann HJ, Fretheim A. Improving the use of research evidence in guideline development: 2. Priority setting. Health Res Policy Syst 2006;4:14

71. Battista RN, Hodge MJ. Setting priorities and selecting topics for clinical practice guidelines. CMAJ 1995;153:1233-7.

72. AGREE Collaboration. Development and validation of an international appraisal instrument for assessing the quality of clinical practice guidelines: the AGREE project. Qual Saf Health Care 2003;12:18-23.

73. Schünemann H, Fretheim A, Oxman A. Improving the use of research evidence in guideline development: 10. Integrating values and consumer involvement. Health Res Policy Syst 2006:4:22.

74. Atkins D, Best D, Briss PA, et al. Grading quality of evidence and strength of recommendations. BMJ 2004;328:1490.

75. Jones J, Hunter D. Qualitative research: consensus methods for medical and health services research. BMJ 1995;311:376-80.

76. Loo R. The Delphi method: a powerful tool for strategic management. Int J Policy Strat Manage 2002;25:762-9.

77. Sullivan WF, Heng J, Cameron D, et al. Consensus guidelines for primary health care of adults with developmental disabilities. Can Fam Physician 2006;52:1410-8.

78. Briss PA, Zasa S, Pappaioanou M, et al. Developing an evidence-based guide to community preventive services — methods. Am J Prev Med 2000;18:35-43.

79. Canadian Task Force on Preventive Health Care. What the Task Force does. Ottawa (ON): The Task Force; 2009. Available: www.canadiantaskforce.ca (accessed 2007 Sept. 25)

80. Benson J, Skull S. Hiding from the sun - vitamin D deficiency in refugees. Aust Fam Physician 2007;36:355-7.

81. Canadian Paediatrics Society. Children and youth new to Canada: a healthcare guide. Ottawa (ON): The Society; 1999.

82. Gushulak BD, MacPherson DW. Population mobility and health: an overview of the relationships between movement and population health. J Travel Med 2004;11: $171-8$

83. Selikowitz HS. Acknowledging cultural differences in the care of refugee and immigrants. Int Dent J 1994;44:59-61.

84. Davis D, Goldman J, Palda VA. Handbook on clinical practice guidelines. Ottawa (ON): Canadian Medical Association; 2007. Available: www.cma.ca//multimedia /CMA/Content Images/CMAInfobase/EN/handbook.pdf (accessed 2009 Sept. 17).

85. Schünemann $\mathrm{H}$, Brozek J, Oxman A, editors. GRADE handbook for grading quality of evidence and strength of recommendations. Version 3.2. The GRADE Working Group; 2009. Available: www.cc-ims.net/gradepro (accessed 2010 June 9)

86. Fervers B, Burgers JS, Haugh MC, et al. Adaptation of clinical guidelines: literature review and proposition for a framework and procedure. Int J Qual Health Care 2006; $18: 167-76$

87. Schünemann HJ, Oxman AD, Vist GE, et al. Chapter 12: Interpreting results and drawing conclusions. In: Higgins JPT, Green S, editors. Cochrane handbook for systematic reviews of interventions. Version 5.0.2. The Cochrane Collaboration; 2009. Available: www.cochrane-handbook.org (accessed 2009 Oct. 17)

88. US Department of Health and Human Services, Agency for Healthcare Research and Quality. Procedure manual [US Preventive Services Task Force]. Publ. No. 08-05118EF. Washington (DC): The Agency; 2008. Available: www.ahrq.gov/clinic/uspstf08 /methods/procmanual.htm (accessed 2010 Jun. 17).

89. Lefebvre C, Manheimer E, Glanville J. Chapter 6: Searching for studies. In: Higgins JPT, Green S, editors. Cochrane handbook for systematic reviews of interventions. Version 5.0.2. The Cochrane Collaboration; 2009. Available: www.cochrane-handbook.org (accessed 2009 Oct. 17).

90. Moher D, Liberati A, Tetzlaff J, et al. Preferred reporting items for systematic reviews and meta-analyses: the PRISMA statement. PLoS Med 2009;6:e1000097.

91. Swann C, Falce C, Morgan A, et al. HDA evidence base. Process and quality standards manual for evidence briefings. 3rd ed. London (UK): Health Development Agency; 2005. Available: www.nice.org.uk/niceMedia/docs/Process_And Quality_Standards_Manual_For_Evidence_Briefings-March2005[1].pdf (accessed 2009 Sept. 17).

92. Cochrane Effective Practice and Organisation of Care Review Group. Data collection checklist. Ottawa (ON): The Cochrane Collaboration; 2002. Available: http://epoc.cochrane.org/sites/epoc.cochrane.org/files/uploads/datacollectionchecklist .pdf (accessed 2007 Sept. 14).

93. Wells GA, Shea B, O'Connell D, et al. Newcastle-Ottawa Scale (NOS) for assessing the quality of non-randomized studies in meta-analyses. Ottawa (ON): The Ottawa Hospital Research Institute; 2007. Available: www.ohri.ca/programs/clinical epidemiology/oxford.htm (accessed 2007 Dec. 13).

94. Glasziou P, Oxman AD, Higgins J. Summary of findings tables within Cochrane reviews: draft specification for RevMan 5.0, December 2004. In: Oxman AD, Glasziou P, Higgins J, editors. Obtaining a consensus on the content and methods of a summary of findings table for Cochrane reviews: report to the Cochrane Collaboration Steering Group. Ottawa (ON): Cochrane Collaboration Steering Group; 2005.

95. Plotkin SA, Orenstein WA, Offit P. Vaccines. 5th ed. Elsevier; 2008.
96. Maciosek MV, Coffield AB, Edwards NM, et al. Priorities among effective clinical preventive services. results of a systematic review and analysis. Am J Prev Med 2006;31:52-61.

97. Roush SW, Murphy DG; Vaccine-Preventable Disease Table Working Group. Historical comparisons of morbidity and mortality for vaccine-preventable diseases in the United States. JAMA 2007;298:2155-63.

98. Peltola H, Kulkarni P, Kapre S, et al. Mumps outbreaks in Canada and the United States: time for new thinking on mumps vaccines. Clin Infect Dis 2007;45:459-66.

99. World Health Organization. Poliomeylitis in Tajikistan — update. Wkly Epidemiol $\operatorname{Rec} 2010 ; 85: 165-6$

100. 2006 Census: immigration and citizenship. Ottawa (ON): Statistics Canada; 2007 Available: http://www12.statcan.ca/census-recensement/2006/rt-td/immcit-eng.cfm (accessed 2011 May 6)

101. World Health Organization. WHO vaccine-preventable diseases: monitoring system. 2004 global summary. Geneva; The Organization; 2004.

102. Walker PF, Jaranson J. Refugee and immigrant health care. Med Clin North Am 1999;83:1103-20.

103. Cheffins T, Chan A, Keane RJ, et al. The impact of rubella immunisation on the incidence of rubella, congenital rubella syndrome and rubella-related terminations of pregnancy in South Australia. Br J Obstet Gynaecol 1998;105:998-1004.

104. McQuillan GM, Kruszon-Moran D, Deforest A, et al. Serologic immunity to diphtheria and tetanus in the United States. Ann Intern Med 2002;136:660-6.

105. Danovaro-Holliday MC, LeBaron CW, Allensworth C, et al. A large rubella outbreak with spread from the workplace to the community. JAMA 2000;284:2733-9.

106. Craig AS, Reed GW, Mohon RT, et al. Neonatal tetanus in the United States: a sentinel event in the foreign-born. Pediatr Infect Dis J 1997;16:955-9.

107. Tiong ACD, Patel MS, Gardiner J, et al. Health issues in newly arrived Afican refugees attending general practice clinics in Melbourne. Med J Aust 2006;185: $602-6$.

108. King A, Varughese P, De Serres G, et al.; Working Group on Measles Elimination. Measles elimination in Canada. J Infect Dis 2004;189(Suppl 1):S236-42.

109. Plotkin S, Orenstein W. Vaccines. 4th ed. Philadelphia (PA): Saunders; 2004

110. Anderson RM. The concept of herd immunity and the design of community-based immunization programs. Vaccine 1992;10:928-35.

111. Public Health Agency of Canada, National Advisory Committee on Immunization. Canadian immunization guide. 7th ed. Ottawa (ON): The Agency; 2006

112. Quinlisk MP. Mumps control today. J Infect Dis 2010;202:655-6.

113. Demicheli V, Jefferson $\mathrm{T}$, Rivetti A, et al. Vaccines for measles, mumps and rubella in children. Cochrane Database Syst Rev 2005;(4):CD004407.

114. Jefferson T, Price D, Demicheli V, et al. Unintended events following immunization with MMR: a systematic review. Vaccine 2003;21:3954-60

115. Wakefield AJ, Murch SH, Anthony A, et al. Ileal-lymphoid-nodular hyperplasia, non-specific colitis, and pervasive developmental disorder in children. Lancet 1998; 351:637-41. Retraction in: Lancet 2010;375:445.

116. Madsen KM, Hviid A, Vestergaard M, et al. Population-based study of measles, mumps, and rubella vaccination and autism. N Engl J Med 2002;347:1477-82.

117. Jefferson T, Rudin M, Di Pietrantonj C. Adverse events after immunisation with aluminium-containing DTP vaccines: systematic review of the evidence. Lancet Infect Dis 2004;4:84-90.

118. Cohen AL, Veenstra D. Economic analysis of prevaccination serotesting compared with presumptive immunization for polio, diphtheria, and tetanus in internationally adopted and immigrant infants. Pediatrics 2006;117:1650-5.

119. Murray TS, Groth ME, Weitzman C, et al. Epidemiology and management of infectious diseases in international adoptees. Clin Microbiol Rev 2005;18:510-20.

120. Barnett ED. Immunizations and infectious disease screening for internationally adopted children. Pediatr Clin North Am 2005;52:1287-309.

121. Guttmann A, Manuel D, Stukel TA, et al. Immunization coverage among young children of urban immigrant mothers: findings from a universal health care system. Ambul Pediatr 2008;8:205-9.

122. Falagas ME, Zarkadoulia E. Factors associated with suboptimal compliance to vaccinations in children in developed countries: a systematic review. Curr Med Res Opin 2008;24:1719-41

123. Skull SA, Ngeow JYY, Hogg G, et al. Incomplete immunity and missed vaccination opportunities in East African immigrants settling in Australia. J Immigr Minor Health 2008;10:263-8.

124. Infectious Diseases Society of America, National and Global Health Committee Immunization Work Group. Executive summary - actions to strengthen adult and adolescent immunization coverage in the United States: policy principles of the Infectious Diseases Society of America. Clin Infect Dis 2007;44:1529-31

125. Figueira M, Christiansen D, Barnett ED. Cost-effectiveness of serotesting compared with universal immunization for varicella in refugee children from six geographic regions. J Travel Med 2003;10:203-7

126. Nysse LJ, Pinsky NA, Bratberg JP, et al. Seroprevalence of antibody to varicell among Somali refugees. Mayo Clin Proc 2007;82:175-80.

127. Knowles SJ, Grundy K, Cahill I, et al. Susceptibility to infectious rash illness in pregnant women from diverse geographical regions. Commun Dis Public Health 2004;7:344-8.

128. Merrett P, Schwartzman K, Rivest P, et al. Strategies to prevent varicella among newly arrived adult immigrants and refugees: a cost-effectiveness analysis. Clin Infect Dis 2007;44:1040-8.

129. Guess HA, Broughton DD, Melton LJ 3rd, et al. Population-based studies of varicella complications. Pediatrics 1986;78:723-7.

130. Gabutti G, Fedele A, Aprile V, et al. Immigration flows and new epidemiological 
evidence in southern Italy. Vaccine 2003;21:399-400

131. Public health dispatch: varicella outbreaks among Mexican adults - Alabama, 2000. Morb Mortal Wkly Rep 2000;49:735-6.

132. Hastie IR. Varicella-zoster virus affecting immigrant nurses. Lancet 1980;2:154-5.

133. Deny P, Leport C, Jestin C. Chickenpox in one hundred Srilankan immigrants in France. Med Mal Infect 1986;16:595-8.

134. Longfield JN, Winn RE, Gibson RL, et al. Varicella outbreaks in army recruits from Puerto Rico. Varicella susceptibility in a population from the tropics. Arch Intern Med 1990;150:970-3.

135. Nguyen HQ, Jumaan AO, Seward JF. Decline in mortality due to varicella after implementation of varicella vaccination in the United States. N Engl J Med 2005; 352:450-8.

136. Zhou F, Harpaz R, Jumaan A, et al. Impact of varicella vaccination on health care utilization. JAMA 2005;294:797-802.

137. Kjersem H, Jepsen S. Varicella among immigrants from the tropics, a health problem. Scand J Soc Med 1990;18:171-4.

138. Danovaro-Holliday MC, Gordon ER, Jumaan AO, et al. High rate of varicella complications among Mexican-born adults in Alabama. Clin Infect Dis 2004;39:1633-39.

139. Pastuszak AL, Levy M, Schick B, et al. Outcome after maternal varicella infection in the first 20 weeks of pregnancy. N Engl J Med 1994;330:901-5.

140. Paryani SG, Arvin AM. Intrauterine infection with varicella-zoster virus after maternal varicella. N Engl J Med 1986;314:1542-6.

141. Zambrano MA, Martinz A, Minguez JA, et al. Varicella pneumonia complicating pregnancy. Acta Obstet Gynecol Scand 1995;74:318-20.

142. Breuer J, Schmid DS, Gershon AA. Use and limitations of varicella-zoster virusspecific serological testing to evaluate breakthrough disease in vaccinees and to screen for susceptibility to varicella. J Infect Dis 2008;197(Suppl 2):S147-51.

143. Holmes CN. Predictive value of a history of varicella infection. Can Fam Physician 2005:51:60-5

144. MacMahon E, Brown LJ, Bexley S, et al. Identification of potential candidates for varicella vaccination by history: questionnaire and seroprevalence study. $B M J$ 2004;329:551-2

145. Seward JF, Marin M, Vazquez M. Varicella vaccine effectiveness in the US vaccination program: a review. J Infect Dis 2008;197:1.

146. Marin M, Guris D, Chaves SS, et al.; Advisory Committee on Immunization Practices, Centers for Disease Control and Prevention. Prevention of varicella: recommendations of the Advisory Committee on Immunization Practices (ACIP). MMWR Recomm Rep 2007;56(RR-4):1-40.

147. National Advisory Committee on Immunization (NACI). Varicella vaccination two-dose recommendations. Can Commun Dis Rep 2010;36(ACS-8):1-36.

148. Chaves SS, Haber P, Walton K, et al. Safety of varicella vaccine after licensure in the United States: experience from reports to the vaccine adverse event reporting system, 1995-2005. J Infect Dis 2008;197(Suppl 2):S170-7.

149. National Advisory Committee on Immunization (NACI). Updated recommendations for the use of varicella and MMR vaccines in HIV-infected individuals. Can Commun Dis Rep 2010;36(ACS-7):1-19.

150. Kwong JC, Tanuseputro P, Zagorski B, et al. Impact of varicella vaccination on health care outcomes in Ontario, Canada: Effect of a publicly funded program? Vaccine 2008;26:6006-12.

151. Rozenbaum MH, Van Hoek AJ, Vegter S, et al. Cost-effectiveness of varicella vaccination programs: an update of the literature. Expert Rev Vaccines 2008;7:753-82.

152. Zhou F, Ortega-Sanchez IR, Guris D, et al. An economic analysis of the universal varicella vaccination program in the United States. J Infect Dis 2008;197(Suppl 2): S156-64.

153. Mills E, Jadad AR, Ross C, et al. Systematic review of qualitative studies exploring parental beliefs and attitudes toward childhood vaccination identifies common barriers to vaccination. J Clin Epidemiol 2005;58:1081-8.

154. Pickering LK, Baker CJ, Freed GL, et al. Immunization programs for infants, children, adolescents and adults: clinical practice guidelines by the Infectious Diseases Society of America. Clin Infect Dis 2009;49:817-40.

155. Jacobson VJC, Szilagyi P. Patient reminder and patient recall systems to improve immunization rates. Cochrane Database Syst Rev 2005;(3):CD003941.

156. Briss PA, Rodewald LE, Hinman AR, et al. Reviews of evidence regarding interventions to improve vaccination coverage in children, adolescents, and adults. Am J Prev Med 2000;18(1 Suppl):97-140.

157. Custer B, Sullivan S, Hazlet T, et al. Global epidemiology of hepatitis B virus. $J$ Clin Gastroenterol 2004;38(Suppl):S158-68.

158. Armstrong L, Goldstein S. Hepatitis B: global epidemiology, diagnosis and prevention. In: Walker P, Barnett E, editors. Immigrant medicine. Philadelphia (PA): Saunders Elsevier; 2007. p. 321-41

159. Public Health Agency of Canada. Immunization \& vaccines: immunization schedules. Ottawa (ON): The Agency; 2008. Available: www.phac-aspc.gc.ca/im/is-vc-eng .php (accessed 2009 Sept. 14)

160. Thompson Coon J, Rogers G, Hewson P, et al. Surveillance of cirrhosis for hepatocellular carcinoma: systematic review and economic analysis. Health Technol Assess 2007;11:1-206.

161. Kao JH, Chen DS. Global control of hepatitis B virus infection. Lancet Infect Dis 2002;2:395-403.

162. Sherman M. Surveillance for hepatocellular carcinoma and early diagnosis. Clin Liver Dis 2007;11:817-37

163. Pohani G, Zou S, Tepper M. Trends of hepatitis B and hepatitis C mortality in Canada 1979-1997. Can J Public Health 2001;92:250-4.

164. ElSaadany S, Tepper M, Mao Y, et al. An epidemiologic study of hepatocellular carcinoma in Canada. Can J Public Health 2002;93:443-6.

165. Gilca V, Duval B, Boulianne N, et al. Impact of the Quebec school-based hepatitis $B$ immunization program and potential benefit of the addition of an infant immunization program. Pediatr Infect Dis J 2006;25:372-4.

166. Manual of clinical microbiology. 9th ed. Washington (DC): ASM Press; 2007.

167. Mandell G, Bennett J, Dolin R. Principles and practice of infectious diseases. 5th ed. Philadelphia (PA): Churchill Livingstone; 2000.

168. Liaw YF, Sung JJ, Chow WC, et al. Lamivudine for patients with chronic hepatitis B and advanced liver disease. N Engl J Med 2004;351:1521-31.

169. Niederau C, Heintges T, Lange S, et al. Long-term follow-up of HBeAg-positive patients treated with interferon alfa for chronic hepatitis B. N Engl J Med 1996;334: 1422-7.

170. Shamliyan TA, MacDonald R, Shaukat A, et al. Antiviral therapy for adults with chronic hepatitis B: a systematic review for a National Institutes of Health Consensus Development conference. Ann Intern Med 2009;150:111-24.

171. Sherman M, Shafran S, Burak KW, et al. Management of chronic hepatitis B: consensus guidelines. Can J Gastroenterol 2007;21(Suppl C):5C-24C.

172. Keeffe EB, Dieterich DT, Han SHB, et al. A treatment algorithm for the management of chronic hepatitis B virus infection in the United States: 2008 update. Clin Gastroenterol Hepatol 2008;6:1315-41.

173. Lok AS, McMahon BJ. AASLD practice guidelines: chronic hepatitis B. Hepatol ogy 2007; $45: 507-39$.

174. Bruix J, Sherman M. Management of hepatocellular carcinoma. Hepatology 2005; 42:1208-36.

175. Zhang BH, Yang BH, Tang ZY. Randomized controlled trial of screening for hepatocellular carcinoma. J Cancer Res Clin Oncol 2004;130:417-22.

176. Lin K, Vickery J. Screening for hepatitis B virus infection in pregnant women: evidence for the US Preventive Services Task Force Reaffirmation Recommendation Statement. Ann Intern Med 2009;150:874-6.

177. Mast EE, Weinbaum CM, Fiore AE, et al.; Advisory Committee on Immunization Practices (ACIP) Centers for Disease Control and Prevention. A comprehensive immunization strategy to eliminate transmission of hepatitis B virus infection in the United States: recommendations of the Advisory Committee on Immunization Practices (ACIP). Part II: immunization of adults. MMWR Morb Mortal Wkly Rep 2006;55(RR-16):1-40.

178. Lee C, Gong Y, Brok J, et al. Hepatitis B immunisation for newborn infants of hepatitis B surface antigen-positive mothers. Cochrane Database Syst Rev 2006; (2):CD004790.

179. Mast EE, Margolis HS, Fiori AE, et al.; Advisory Committee on Immunization Practices (ACIP). A comprehensive immunization strategy to eliminate transmission of hepatitis B virus infection in the United States: recommendations of the Advisory Committee on Immunization Practices (ACIP). Part 1: immunization of infants, children, and adolescents. MMWR Recomm Rep 2005;54(RR-16):1-39.

180. McMahon BJ, Rhoades ER, Heyward W, et al. A comprehensive programme to reduce the incidence of hepatitis B virus infection and its sequelae in Alaskan natives. Lancet 1987; 2:1134-6.

181. Wilson N, Ruff T, Jung Rana B, et al. The effectiveness of the infant hepatitis B immunisation program in Fiji, Kiribati, Tonga and Vanuatu. Vaccine 2000;18: 3059-66.

182. Ni YH, Chang MH, Huang LM, et al. Hepatitis B virus infection in children and adolescents in a hyperendemic area: 15 years after mass hepatitis B vaccination. Ann Intern Med 2001;135:796-800.

183. Del Poggio P, Mazzoleni M. Screening in liver disease. World J Gsatroenterol 2006; $12: 5272-80$

184. Chang MH, Chen CJ, Lai MS, et al. Universal hepatitis B vaccination in Taiwan and the incidence of hepatocellular carcinoma in children. N Engl J Med 1997;336: 1855-9.

185. Chen W, Gluud C. Vaccines for preventing hepatitis B in health care workers Cochrane Database Syst Rev 2005;(4):CD000100.

186. Yuan L, Robinson G. Hepatitis B vaccination and screening for markers at a sexually transmitted disease clinic for men. Can J Public Health 1994;85:338-41.

187. Blostein J, Clark P. Cost-effectiveness of preimmunization hepatitis B screening in high-risk adolescents. Public Health Rep 2001;116:165-8.

188. Hutton DW, Tan D, So SK, et al. Cost-effectiveness of screening and vaccinating Asian and Pacific Islander adults for hepatitis B. Ann Intern Med 2007;147:460-9.

189. Huerga H, Lopez-Velez R. Infectious diseases in sub-Saharan African immigrant children in Madrid, Spain. Pediatr Infect Dis J 2002;21:830-4

190. Hayes EB, Talbot SB, Matheson ES, et al. Health status of pediatric refugees in Portland, Me. Arch Pediatr Adolesc Med 1998;152:564-8.

191. Hislop TG, Teh C, Low A, et al. Hepatitis B knowledge, testing and vaccination levels in Chinese immigrants to British Columbia, Canada. Can J Public Health 2007;98:125-9.

192. Nguyen TT, Taylor V, Chen MS Jr, et al. Awareness, knowledge, and screening among Asian Americans. J Cancer Educ 2007;22:266-72.

193. Vryheid RE. A survey of vaccinations of immigrants and refugees in San Diego County, California. Asian Am Pac Isl J Health 2001;9:221-30.

194. Kim YO. Access to hepatitis B vaccination among Korean American children in immigrant families. J Health Care Poor Underserved 2004;15:170-82.

195. Kim YOR, Telleen S. Predictors of hepatitis B immunization status in Korean American children. J Immigr Health 2001;3:181-92.

196. Centers for Disease Control and Prevention. Recommendations for identification and public health management of person with chronic hepatitis B virus infection. MMWR Morb Mortal Wkly Rep 2008;57(RR-8):1-20. 
197. Menzies D, Oxlade O, Lewis M. Costs for tuberculosis care in Canada. Ottawa (ON): Public Health Agency of Canada; 2006

198. Canadian tuberculosis standards. 6th ed. Ottawa (ON): Tuberculosis Prevention and Control, Public Health Agency of Canada, Canadian Lung Association/Canadian Thoracic Society; 2007.

199. World Health Organization. WHO Report 2008. Global tuberculosis control. Surveillance, planning, financing. Geneva (Switzerland): The Organization; 2008.

200. Public Health Agency of Canada. Tuberculosis in Canada 2004. Ottawa (ON): The Agency; 2007.

201. Tuberculosis in Canada 2000. Ottawa (ON): Health Canada, Tuberculosis and Bacterial Respiratory Diseases; 2003.

202. Cain KP, Haley CA, Armstrong LR, et al. Tuberculosis among foreign-born persons in the United States: achieving tuberculosis elimination. Am J Respir Crit Care Med 2007;175:75-9.

203. Creatore MI, Lam M, Wobeser WL. Patterns of tuberculosis risk over time among recent immigrants to Ontario, Canada. Int J Tuberc Lung Dis 2005;9:667-72.

204. Farah MG, Meyer HE, Selmer R, et al. Long-term risk of tuberculosis among immigrants in Norway. Int J Epidemiol 2005;34:1005-11.

205. Watkins RE, Brennan R, Plant AJ. Tuberculin reactivity and the risk of tuberculosis: a review. Int J Tuberc Lung Dis 2000;4:895-903

206. Morrison J, Pai M, Hopewell PC. Tuberculosis and latent tuberculosis infection in close contacts of people with pumonary tuberculosis in low-income and middleincome countries: a systematic review and meta-analysis. Lancet Infect Dis 2008;8: 359-68.

207. Tuberculosis among Indochinese refugees — an update. MMWR Morb Mortal Wkly Rep 1981;30:603-6.

208. Enarson DA. Active tuberculosis in Indochinese refugees in British Columbia. CMAJ 1984;131:39-42.

209. Wilcke JT, Poulsen S, Askgaard DS, et al. Tuberculosis in a cohort of Vietnamese refugees after arrival in Denmark 1979-1982. Int J Tuberc Lung Dis 1998;2:219-24.

210. Thorpe LE, Laserson K, Cookson S, et al. Infectious tuberculosis among newly arrived refugees in the United States. N Engl J Med 2004;350:2105-6.

211. Marras TK, Wilson J, Wang EEL, et al. Tuberculosis among Tibetan refugee claimants in Toronto: 1998 to 2000. Chest 2003;124:915-21

212. Menzies D, Gardiner G, Farhat M, et al. Thinking in three dimensions: a web-based algorithm to aid the interpretation of tuberculin skin test results. Int J Tuberc Lung Dis 2008;12:498-505.

213. Pai M, Zwerling A, Menzies D. Systematic review: T-cell-based assays for the diagnosis of latent tuberculosis infection: an update. Ann Intern Med 2008;149:177-84.

214. Farhat M, Greenaway C, Pai M, et al. False-positive tuberculin skin tests: What is the absolute effect of BCG and non-tuberculous mycobacteria? Int J Tuberc Lung Dis 2006;10:1192-204

215. Marcus JH, Khassis Y. Tuberculin sensitivity in BCG vaccinated infants and children in Israel. Acta Tuberc Pneumol Scand 1965;46:113-22.

216. Karalliede S, Katugha LP, Uragoda CG. Tuberculin response of Sri Lankan children after BCG vaccination at birth. Tubercle 1987;68:33-8.

217. Reid JK, Ward H, Marciniuk D, et al. The effect of neonatal BCG vaccination on PPD testing on Canadian Aboriginal children. Chest 2007;131:1806-10.

218. Sepulveda RL, Ferrer X, Latrach C, et al. The influence of Calmette-Guérin bacillus immunization on the booster effect of tuberculin testing in healthy young adults. Am Rev Respir Dis 1990;142:24-8.

219. Updated recommendations on interferon gamma release assays for latent tuberculosis infection. An Advisory Committee Statement (ACS). Can Commun Dis Rep 2008;34:1-13.

220. Oxlade O, Schwartzman K, Menzies D. Interferon-gamma release assays and TB screening in high-income countries: a cost-effectiveness analysis. Int J Tuberc Lung Dis 2007;11:16-26.

221. Smieja MJ, Marchetti CA, Cook DJ, et al. Isoniazid for preventing tuberculosis in non-HIV infected persons. Cochrane Database Syst Rev 1999;(1):CD001363.

222. Bucher HC, Griffith LE, Guyatt GH, et al. Isoniazid prophylaxis for tuberculosis in HIV infection: a meta-analysis of randomized controlled trials. AIDS 1999;13:501-7.

223. International Union Against Tuberculosis Committee on Prophylaxis. Efficacy of various durations of isoniazid preventive therapy for tuberculosis: five years of follow-up in the IUAT trial. Bull World Health Organ 1982;60:555-64.

224. Fountain FF, Tolley E, Chrsman CR, et al. Isoniazid hepatotoxicity associated with treatment of latent tuberculosis infection: a 7-year evaluation from a public health tuberculosis clinic. Chest 2005;128:116-23.

225. Comstock GW. How much isoniazid is needed for prevention of tuberculosis among immunocompetent adults? Int J Tuberc Lung Dis 1999;3:847-50.

226. Public Health Agency of Canada. Tuberculosis drug resistance in Canada 2007. Ottawa (ON): The Agency; 2007.

227. Khan K, Muennig P, Behta M, et al. Global drug-resistance patterns and the management of latent tuberculosis infection in immigrants to the United States. $N$ Engl J Med 2002;347:1850-9.

228. Nolan CM, Goldberg SV, Buskin SE. Hepatotoxicity associated with isoniazid preventive therapy: a 7-year survey from a public health tuberculosis clinic. JAMA 1999;281:1014-8.

229. LoBue PA, Moser KS. Use of isoniazid for latent tuberculosis infection in a public health clinic. Am J Respir Crit Care Med 2003;168:443-7.

230. Pediatric Tuberculosis Collaborative Group. Targeted tuberculin skin testing and treatment of latent tuberculosis infection in children and adolescents. Pediatrics 2004;114:1175-201.

231. Saukkonen J, Cohn D, Jasmer R, et al. An official ATS statement: hepatotoxicity of antituberculosis therapy. Am J Respir Crit Care Med 2006;174:935-52

232. Mandalakas AM, Starke JR. Current concepts of childhood tuberculosis. Semin Pediatr Infect Dis 2005;16:93-104.

233. Carvalho AC, Saleri N, El-Hamad I, et al. Completion of screening for latent tuberculosis infection among immigrants. Epidemiol Infect 2005;133:179-85.

234. Wyss LL, Alderman MK. Using theory to interpret beliefs in migrants diagnosed with latent TB. Online J Issues Nurs 2006;12:7.

235. Brewin P, Jones A, Kelly M, et al. Is screening for tuberculosis acceptable to immigrants? A qualitative study. J Public Health (Oxf) 2006;28:253-60.

236. Coreil J, Lauzardo M, Heurtelou M. Cultural feasibility assessment of tuberculosis prevention among persons of Haitian origin in South Florida. J Immigr Health 2004; 6:63-9.

237. Griffiths C, Sturdy P, Brewin P, et al. Educational outreach to promote screening for tuberculosis in primary care: a cluster randomised controlled trial. Lancet 2007; 369:1528-34.

238. Ailinger RL, Dear MR. Adherence to tuberculosis preventive therapy among Latino immigrants. Public Health Nurs 1998;15:19-24.

239. Catlos EK, Cantwell MF, Bhatia G, et al. Public health interventions to encourage TB class A/B1/B2 immigrants to present for TB screening. Am J Respir Crit Care Med 1998; 158:1037-41.

240. Goldberg SV, Wallace J, Jackson JC, et al. Cultural case management of latent tuberculosis infection. Int J Tuberc Lung Dis 2004;8:76-82.

241. AIDS epidemic update 2007. Geneva (CH): Joint United Nations Programme on HIV/AIDS (UNAIDS) and World Health Organization; 2007. Available: http://data.unaids.org/pub/EPISlides/2007/2007_epiupdate_en.pdf (accessed 2010 June 1).

242. Public Health Agency of Canada. Population-specific HIV/AIDS status report: people from countries where HIV is endemic - Black people of African and Caribbean descent living in Canada. Ottawa (ON): The Agency; 2009. Available: www.phac-aspc.gc.ca/aids-sida/publication/ps-pd/africacaribbe/frw-avpro-eng.php (accessed 2010 June 24).

243. Public Health Agency of Canada, Centre for Infectious Disease Prevention and Control, Surveillance and Risk Assessment Division. HIV/AIDS epi updates 2006. Ottawa (ON): The Agency; 2006. Available: www.phac-aspc.gc.ca/publicat/epiu-aepi/epi-06 /index-eng.php (accessed 2010 June 1).

244. Adrien A, Leaune V, Remis RS, et al. Migration and HIV: an epidemiological study of Montréalers of Haitian origin. Int J STD AIDS 1999;10:237-42.

245. Spiegel PB, Bennedsen AR, Claass J, et al. Prevalence of HIV infection in conflictaffected and displaced people in seven sub-Saharan African countries: a systematic review. Lancet 2007;369:2187-95.

246. Rosen S, Fox MP, Gill CJ. Patient retention in antiretroviral therapy programs in sub-Saharan Africa: a systematic review. PLoS Med 2007;4:e298.

247. Siegfried NL, Van Deventer PJU, Mahomed FA, et al. Stavudine, lamivudine and nevirapine combination therapy for treatment of HIV infection and AIDS in adults. Cochrane Database Syst Rev 2006;(2):CD004535.

248. US Preventive Services Task Force. Screening for HIV: recommendation statement. Ann Intern Med 2005;143:32-7.

249. Weinhardt LS, Carey MP, Johnson BT, et al. Effects of HIV counseling and testing on sexual risk behavior: a meta-analytic review of published research, 1985-1997. Am J Public Health 1999;89:1397-405.

250. Elwy AR, Hart GJ, Hawkes S, et al. Effectiveness of interventions to prevent sexually transmitted infections and human immunodeficiency virus in heterosexual men - a systematic review. Arch Intern Med 2002;162:1818-30.

251. HIV Trialists Collaborative Group. Zidovudine, didanosine, and zalcitabine in the treatment of HIV infection: meta-analyses of the randomised evidence. Lance 1999;353:2014-25

252. Ioannidis JPA, Collier AC, Cooper DA, et al. Clinical efficacy of high-dose acyclovir in patients with human immunodeficiency virus infection: a meta-analysis of randomized individual patient data. J Infect Dis 1998;178:349-59.

253. Rogstad KE, Palfreeman A, Rooney G, et al. United Kingdom national guidelines on HIV testing. London (UK): British Association for Sexual Health and HIV, Clinical Effectiveness Group; 2006.

254. Crepaz N, Hart TA, Marks G. Highly active antiretroviral therapy and sexual risk behavior - a meta-analytic review. JAMA 2004;292:224-36.

255. Burdge DR, Money DM, Forbes JC, et al. Canadian consensus guidelines for the management of pregnancy, labour and delivery and for postpartum care in HIVpositive pregnant women and their offspring (summary of 2002 guidelines). CMAJ 2003; $168: 1671-4$

256. Enanoria WTA, Ng C, Saha SR, et al. Treatment outcomes after highly active antiretroviral therapy: a meta-analysis of randomised controlled trials. Lancet Infect Dis 2004;4:414-25

257. Merson MH, Dayton JM, O'Reilly K. Effectiveness of HIV prevention interventions in developing countries. AIDS 2000;14:S68-84

258. Samson L, King S. Evidence-based guidelines for universal counselling and offering of HIV testing in pregnancy in Canada. CMAJ 1998;158:1449-57.

259. Branson BM, Handsfield HH, Lampe MA, et al. Revised recommendations for HIV testing of adults, adolescents, and pregnant women in health-care settings. MMWR Recomm Rep 2006;55:1-17.

260. Palella FJ, Delaney KM, Moorman AC, et al. Declining morbidity and mortality among patients with advanced human immunodeficiency virus infection. $N$ Engl J Med 1998;338:853-60.

261. Riddler SA, Smit E, Cole SR, et al. Impact of HIV infection and HAART on serum lipids in men. JAMA 2003;289:2978-82. 
262. Zencovich M, Kennedy K, MacPherson DW, et al. Immigration medical screening and HIV infection in Canada. Int J STD AIDS 2006;17:813-6.

263. Public Health Agency of Canada, Surveillance and Risk Assessment Division. HIV/AIDS in Canada. Surveillance report to December 31, 2006. Ottawa (ON): The Agency; 2007.

264. amfAR (Foundation for AIDS Research). Women and HIV/AIDS. Fact sheet no. 2. Washington (DC): The Foundation, Public Policy Office; 2008.

265. Welz T, Hosegood V, Jaffer S, et al. Continued very high prevalence of HIV infection in rural Kwazulul-Natal, South Africa: a population-based longitudinal study. AIDS 2007;21:1467-72.

266. Rodriguez C. Sexual violence in South Kivu, Congo. Forced Migr Rev 2007;27:45-6.

267. Martino JL, Vermund SH. Vaginal douching: evidence for risks or benefits to women's health. Epidemiol Rev 2002;24:109-24

268. Castilla J, del Romero J, Hernando V, et al. Effectiveness of highly active antiretroviral therapy in reducing heterosexual transmission of HIV. J Acquir Immune Defic Syndr 2005:40:96-101.

269. Paltiel AD, Weinstein MC, Kimmel AD, et al. Expanded screening for HIV in the United States: an analysis of cost-effectiveness. N Engl J Med 2005;352:586-95.

270. Lawson E, Gardezi F, Calzavara L, et al. HIV/AIDS stigma, denial, fear and discrimination, experiences and responses of people from African and Caribbean communities in Toronto. Toronto (ON): African and Caribbean Council on HIV/AIDS in Ontario and University of Toronto, HIV Social, Behavioural and Epidemiological Studies Unit; 2006.

271. Vermund SH, Wilson CM. Barriers to HIV testing - Where next? Lancet 2002; 360:1186-7.

272. Herek GM, Capitanio JP, Widaman KF. Stigma, social risk, and health policy: public attitudes toward HIV surveillance policies and the social construction of illness. $J$ Health Psychol 2003;22:533-40.

273. Tharao E, Massaquio N, Teclom S. The silent voices of HIV/AIDS epidemic: African and Caribbean women in Toronto 2002-2004. Toronto (ON): Women's Health in Women's Hands; 2006.

274. Te HS, Jensen DM. Epidemiology of hepatitis B and C viruses: a global overview. Clin Liver Dis 2010;14:1-21.

275. Remis R. A study to characterize the epidemiology of hepatitis $C$ infection in Canada, 2002. Ottawa (ON): Health Canada, Population and Public Health Branch, Centre for Infectious Disease Prevention and Control, Hepatitis C Prevention Support and Research Program; 2003.

276. Nordenstedt H, White D, El-Serag $\mathrm{H}$. The changing pattern of epidemiology in hepatocellular carcinoma. Dig Liver Dis 2010;42:S206-14.

277. Sherman M, Shafran S, Burak KW, et al. Management of chronic hepatitis C: consensus guidelines. Can J Gastroenterol 2007;21(Suppl C):25C-34C.

278. Ghany MG, Strader DB, Thomas DL, et al.; American Association for the Study of Liver D. Diagnosis, management, and treatment of hepatitis C: an update. Hepatology 2009;49:1335-74

279. Asselah T, Marcellin P. New direct-acting antivirals combination for the treatment of chronic hepatitis C. Liver Int 2011;31(Suppl 1):68-77.

280. Manzardo C, Treviño B, Cabezos J, et al. Communicable diseases in the immigrant population attended to in a tropical medicine unit: epidemiological aspects and public health issues. Travel Med Infect Dis 2008;6:4-11.

281. Batash S, Khaykis I, Raicht RF, et al. High prevalence of hepatitis C virus infection among immigrants from the former Soviet Union in the New York City metropoli$\tan$ area: results of a community-based screening program. Am J Gastroenterol 2008;103:922-7

282. Lopez-Velez R, Huerga H, Turrientes MC. Infectious diseases in immigrants from the perspective of a tropical medicine referral unit. Am J Trop Med Hyg 2003;69: 115-21

283. World Health Organization. Global prevalence of hepatitis A, B and C. Wkly Epidemiol Rec 2002;77:41-8.

284. World Health Organization. Hepatitis C - global prevalence (update). Wkly Epidemiol Rec 1999;74:425-7.

285. Hauri AM, Armstrong G, Hutin YJF. The global burden of disease attributable to contaminated injections given in the health care setting. Int J STD AIDS 2004;15:7-16.

286. Missiha SB, Ostrowski M, Heathcote EJ. Disease progression in chronic hepatitis C: modifiable and nonmodifiable factors. Gastroenterology 2008;134:1699-714.

287. Sherman M, Bain V, Villeneuve JP, et al. The management of chronic viral hepatitis: a Canadian consensus conference 2004. Can J Gastroenterol 2004;18:715-28.

288. Shepherd J, Jones J, Hartwell D, et al. Interferon alfa (pegylated and nonpegylated) and ribavirin for the treatment of mild chronic hepatitis $\mathrm{C}$ : a systematic review and economic evaluation. Health Technol Assess 2007;11:1-205

289. Hartwell D, Shepherd J. Pegylated and non-pegylated interferon-alfa and ribavirin for the treatment of mild chronic hepatitis $\mathrm{C}$ : a systematic review and meta-analysis. Int J Technol Assess Health Care 2009;25:56-62.

290. Simin M, Brok J, Stimac D, et al. Cochrane systematic review: pegylated interferon plus ribavirin vs. interferon plus ribavirin for chronic hepatitis C. Aliment Pharmacol Ther 2007;25:1153-62

291. Shepherd J, Brodin H, Cave C, et al. Pegylated interferon alpha-2a and -2b in combination with ribavirin in the treatment of chronic hepatitis $\mathrm{C}$ : a systematic review and economic evaluation. Health Technol Assess 2004;8:iii-iv, 1-125.

292. Bruno S, Stroffolini T, Colombo M, et al. Sustained virological response to interferon-alpha is associated with improved outcome in HCV-related cirrhosis: a retrospective study. Hepatology 2007:45:579-87.

293. Veldt BJ, Heathcote J, Wedemeyer H, et al. Sustained virologic response and clinical outcomes in pateints with chronic hepatitis $\mathrm{C}$ and advanced fibrosis. Ann Intern
Med 2007; 147:677-84.

294. Alberti A. Impact of a sustained virological response on the long-term outcome of hepatitis C. Liver Int 2011;31(Suppl 1):18-22.

295. Andriulli A, Mangia A, Iacobellis A, et al. Meta-analysis: the outcome of anti-vira therapy in HCV genotype 2 and genotype 3 infected patients with chronic hepatitis. Aliment Pharmacol Ther 2008;28:397-404.

296. Petta S, Craxi A. Optimal therapy in hepatitis C virus gneotypes 2 and 3 patients Liver Int 2011;31(Suppl 1):36-44.

297. Kamal SM. Hepatitis C virus genotype 4 therapy: progress and challenges. Liver Int 2011;31(Suppl 1):45-52.

298. Antaki N, Craxi A, Kamal Sanaa M, et al. The neglected hepatitis C virus genotypes 4, 5 and 6: an international consensus report. Liver Int 2010;30:342-55.

299. Borroni G, Andreoletti M, Casiraghi M, et al. Effectiveness of pegylated interferon/ribavirin combination in "real world" patients with chronic hepatitis C virus infection. Aliment Pharmacol Ther 2008;27:790-7.

300. Zeuzem S. Heterogeneous virologic response rates to interferon-based therapy in patients with chronic hepatitis C: Who responds less well? Ann Intern Med 2004; 140:370-81.

301. Heathcote EJ, Shiffman ML, Cooksley WG, et al. Peginterferon alfa-2a in patients with chronic hepatitis C and cirrhosis. N Engl J Med 2000;343:1673-80.

302. Gane E. Future hepatitis C virus treatment: interferon-sparing combinations. Liver Int 2011;31(Suppl 1):62-7.

303. Sroczynski G, Esteban E, Conrads-Frank A, et al. Long-term effectiveness and cost-effectiveness of screening for hepatitis C virus infection. Eur J Public Health 2009; 19:245-53.

304. Plunkett BA, Grobman WA. Routine hepatitis C virus screening in pregnancy: a cost-effectiveness analysis. Am J Obstet Gynecol 2005; 192:1153-61.

305. Singer ME, Younossi ZM. Cost effectiveness of screening for hepatitis $C$ virus in asymptomatic, average-risk adults. Am J Med 2001;111:614-21.

306. Caruana SR, Kelly HA, De Silva SL, et al. Knowledge about hepatitis and previous exposure to hepatitis viruses in immigrants and refugees from the Mekong region. Aus N Z J Public Health 2005;29:64-8.

307. O'Connor CC, Shaw M, Wen LM, et al. Low knowledge and high infection rates of hepatitis in Vietnamese men in Sydney. Sex Health 2008;5:299-302.

308. Zickmund SL, Brown KE, Bielefeldt K. A systematic review of provider knowledge of hepatitis C: Is it enough for a complex disease? Dig Dis Sci 2007;52:2550-6.

309. Shehab TM, Sonnad SS, Lok AS. Management of hepatitis C patients by primary care physicians in the USA: results of a national survey. $J$ Viral Hepat 2001;8:377-83.

310. Cozzolongo R, Cuppone R, Petruzzi J, et al. Approach of primary care physician to hepatitis C: an educational survey from a southern Italian area. J Infect 2005;51: 396-400.

311. Ferrante JM, Winston D, Chen P, et al. Family physicians' knowledge and screening of chronic hepatitis and liver cancer. Fam Med 2008;40:345-51.

312. Chou R, Clark EC, Helfand M, et al. Screening for hepatitis C virus infection: a review of the evidence for the U.S. Preventive Services Task Force. Ann Intern Med 2004;140:465-79.

313. Boulware DR, Stauffer WM, Hendel-Paterson BR, et al. Maltreatment of Strongyloides infection: case series and worldwide physicians-in-training survey. Am J Med 2007;120:545 .e1-8.

314. Genta RM. Predictive value of an enzyme-linked immunosorbent assay (ELISA) for the serodiagnosis of strongyloidiasis. Am J Clin Pathol 1988;89:391-4.

315. Sudarshi S, Stumpfle R, Armstrong M, et al. Clinical presentation and diagnostic sensitivity of laboratory tests for Strongyloides stercoralis in travellers compared with immigrants in a non-endemic country. Trop Med Int Health 2003;8:728-32.

316. Gyorkos TW, Genta RM, Viens P, et al. Seroepidemiology of strongyloides infection in the Southeast Asian refugee population in Canada. Am J Epidemiol 1990; 132:257-64.

317. Whitty CJ, Mabey DC, Armstrong M, et al. Presentation and outcome of 1107 cases of schistosomiasis from Africa diagnosed in a nonendemic country. Trans $R$ Soc Trop Med Hyg 2000;94:531-4.

318. Kumar V, Gryseels B. Use of praziquantel against schistosomiasis: a review of current status. Int J Antimicrob Agents 1994;4:313-20.

319. Saconato H, Atallah A. Interventions for treating schistosomiasis mansoni Cochrane Database Syst Rev 2000;(2):CD000528.

320. Toma H. Comparative studies on the efficacy of three anthelminthics on treatment of human strongyloidiasis in Okinawa, Japan. Southeast Asian J Trop Med Public Health 2000;31:147-51.

321. Refugee health guidelines: domestic guidelines. Guidelines for evaluation of refugees for intestinal and tissue-invasive parasitic infections during domestic medical examination. Atlanta (GA): Centers for Disease Control and Prevention. Available: http://198.246.98.21/immigrantrefugeehealth/pdf/intestinal-parasites-domestic .pdf (accessed 2011 May 5)

322. Buchwald D, Lam M, Hooton TM. Prevalence of intestinal parasites and association with symptoms in Southeast Asian refugees. J Clin Pharm Ther 1995;20:271-5.

323. Lifson AR, Thai D, O'Fallon A, et al. Prevalence of tuberculosis, hepatitis B virus, and intestinal parasitic infections among refugees to Minnesota. Public Health Rep 2002;117:69-77.

324. Martin JA, Mak DB. Changing faces: a review of infectious disease screening of refugees by the Migrant Health Unit, Western Australia in 2003 and 2004. Med J Aust 2006;185:607-10.

325. McLeod A, Reeve M. The health status of quota refugees screened by New Zealand's Auckland Public Health Service between 1995 and 2000. N Z Med J 2005;118:U1702. 
326. Miller JM, Boyd HA, Ostrowski SR, et al. Malaria, intestinal parasites, and schistosomiasis among Barawan Somali refugees resettling to the United States: a strategy to reduce morbidity and decrease the risk of imported infections. Am J Trop Med Hyg 2000;62:115-21.

327. Molina CD, Molina MM, Molina JM. Intestinal parasites in Southeast Asian refugees two years after immigration. West J Med 1988;149:422-5.

328. Brodine SK, Thomas A, Huang R, et al. Community based parasitic screening and treatment of Sudanese refugees: application and assessment of Centers for Disease Control guidelines. Am J Trop Med Hyg 2009;80:425-30.

329. Caruana SR, Kelly HA, Ngeow JY, et al. Undiagnosed and potentially lethal parasite infections among immigrants and refugees in Australia. J Travel Med 2006;13: 233-9.

330. de Silva S, Saykao P, Kelly H, et al. Chronic strongyloides stercoralis infection in Laotian immigrants and refugees 7-20 years after resettlement in Australia. Epidemiol Infect 2002;128:439-44.

331. Posey DL, Blackburn BG, Weinberg M, et al. High prevalence and presumptive treatment of schistosomiasis and strongyloidiasis among African refugees. Clin Infect Dis 2007;45:1310-5.

332. Rice JE, Skull SA, Pearce C, et al. Screening for intestinal parasites in recently arrived children from East Africa. J Paediatr Child Health 2003;39:456-9.

333. Seybolt LM, Christiansen D, Barnett ED. Diagnostic evaluation of newly arrived asymptomatic refugees with eosinophilia. Clin Infect Dis 2006;42:363-7.

334 Franco-Paredes C, Dismukes R, Nicolls D, et al. Persistent and untreated tropical infectious diseases among Sudanese refugees in the United States. Am J Trop Med Hyg 2007;77:633-5.

335. Chitsulo L, Engels D, Montresor A, et al. The global status of schistosomiasis and its control. Acta Trop 2000;77:41-51.

336. Garg PK, Perry S, Dorn M, et al. Risk of intestinal helminth and protozoan infection in a refugee population. Am J Trop Med Hyg 2005;73:386-91.

337. Davis J, Webber M. A prospective audit of 215 newly arrived African refugees. $J$ Paediatr Child Health 2007;42(Suppl 1):A11.

338. Gibney KB, Mihrshahi S, Torresi J, et al. The profile of health problems in African immigrants attending an infectious disease unit in Melbourne, Australia. Am J Trop Med Hyg 2009;80:805-11.

339. Nielsen PB, Mojon M. Improved diagnosis of Strongyloides stercoralis by seven consecutive stool specimens. Zentralbl Bakteriol Mikrobiol Hyg [A] 1987;263:616-8.

340. Karunajeewa H, Kelly H, Leslie D, et al. Parasite-specific IgG response and peripheral blood eosinophil count following albendazole treatment for presumed chronic strongyloidiasis. J Travel Med 2006;13:84-91

341. Kobayashi J, Sato Y, Toma H, et al. Application of enzyme immunoassay for postchemotherapy evaluation of human strongyloidiasis. Diagn Microbiol Infect Dis 1994;18:19-23.

342. Lindo JF, Atkins NS, Lee MG, et al. Short report: long-term serum antibody isotype responses to Strongyloides stercoralis filariform antigens in eight patients treated with ivermectin. Am J Trop Med Hyg 1996;55:474-6.

343. Loutfy MR, Wilson M, Keystone JS, et al. Serology and eosinophil count in the diagnosis and management of strongyloidiasis in a non-endemic area. Am J Trop Med Hyg 2002;66:749-52.

344. Drugs for parasitic infections. New Rochelle (NY): The Medical Letter Inc.; 2007

345. Lim S, Katz K, Krajden S, et al. Complicated and fatal Strongyloides infection in Canadians: risk factors, diagnosis and management. CMAJ 2004;171:479-84.

346. Lagacé-Wiens PR, Harding GK. A Canadian immigrant with coinfection of Strongyloides stercoralis and human T-lymphotropic virus 1. CMAJ 2007;177:451-3.

347. Drugs and health products [database] Ottawa (ON): Health Canada; 2008 Available: www.hc-sc.gc.ca/dhp-mps/acces/drugs-drogues/index-eng.php (accessed 2010 Nov. 1).

348. World Health Organization. Guidelines for the treatment of malaria. 2nd ed. Geneva (Switzerland): The Organization; 2010.

349. Taylor SM, Molyneux ME, Simel DL, et al. Does this patient have malaria? JAMA 2010;304:2048-56.

350. Canadian recommendations for the prevention and treatment of malaria among international travellers. Can Commun Dis Rep 2009;35(Suppl 1):1-82.

351. Mali S, Steele S, Slutsker L, et al. Malaria surveillance - United States, 2007. MMWR Surveill Summ 2009;58(SS02):1-16.

352. Monge-Maillo B, Jiménez C, Pérez-Molina JA, et al. Imported infectious diseases in mobile populations, Spain. Emerg Infect Dis 2009;15:1745-52.

353. Prematunga C, McCarthy A. Severe malaria surveillance and treatment delays in Canada [abstract 51.024]. Int J Infect Dis 2010;14(Supp 1):e211.

354. Kain KC, MacPherson DW, Kelton T, et al. Malaria deaths in visitors to Canada and in Canadian travellers: a case series. CMAJ 2001;164:654-9.

355. Lavoie PM, Carceller A, Robert M, et al. Malaria in immigrant and traveler children: clinical presentation and risk factors for complications. J Pediatr Infect Dis 2006;1:29-37.

356. Skarbinski J, Eliades J, Causer LM, et al. Malaria surveillance - United States, 2004. MMWR Surveill Summ 2006;55(SS04):23-37.

357. Thwing J, Skarbinski J, Newman RD, et al. Malaria surveillance — United States. MMWR Surveill Summ 2007;56(SS06):23-40.

358. Dal-Bianco MP, Koster KB, Kombila UD, et al. High prevalence of asymptomatic Plasmodium falciparum infection in Gabonese adults. Am J Trop Med Hyg 2007;77:939-42.

359. Marangi M, Di Tullio R, Mens PF, et al. Prevalence of Plasmodium spp. in malaria asymptomatic African migrants assessed by nucleic acid sequence based amplification. Malar J 2009;8:12.
360. Njama-Meya D, Kamya MR, Dorsey G. Asymptomatic parasitaemia as a risk factor for symptomatic malaria in a cohort of Ugandan children. Trop Med Int Health 2004;9:862-8.

361. Stauffer WM, Newberry AM, Cartwright CP, et al. Evaluation of malaria screening in newly arrived refugees to the United States by microscopy and rapid antigen capture enzyme assay. Pediatr Infect Dis $J$ 2006;25:948-50.

362. Omari AAA, Gamble CL, Garner P. Artemether-lumefantrine (six-dose regimen) for treating uncomplicated falciparum malaria. Cochrane Database Syst Rev 2005; (4):CD005564.

363. Osei-Akoto AO, Owusu-Ofori S. Atovaquone-proguanil for treating uncomplicated malaria. Cochrane Database Syst Rev 2005;(4):CD004529.

364. Maroushek SR, Aguilar EF, Stauffer W, et al. Malaria among refugee children at arrival in the United States. Pediatr Infect Dis $J$ 2005;24:450-2

365. Olliaro P, Pinoges L, Checchi F, et al. Risk associated with asymptomatic parasitaemia occurring post-antimalarial treatment. Trop Med Int Health 2008;13:83-90.

366. Benson J, Davis J. Malaria in the Australian refugee population. Aust Fam Physician 2007;36:639-41.

367. Stauffer WM, Weinberg M. Emerging clinical issues in refugees. Curr Opin Infect Dis 2009;22:436-42.

368. Miller K, Banerji A. Epidemiology of malaria presenting at British Columbia's Children's Hospital, 1984-2001: lessons for prevention. Can J Public Health 2004:95:245-8.

369. Wongsrichanalai C, Barcus MJ, Muth S, et al. A review of malaria diagnostic tools: microscopy and rapid diagnostic test (RDT). Am J Trop Med Hyg 2007;77(Suppl 6): 119-27.

370. Murray CK, Gasser RAJ, Magill AJ, et al. Update on rapid diagnostic testing for malaria. Clin Microbiol Rev 2008;21:97-110.

371. Mens P, Spieker N, Omar S, et al. Is molecular biology the best alternative for diag nosis of malaria to microscopy? A comparison between microscopy, antigen detection and molecular tests in rural Kenya and urban Tanzania. Trop Med Int Health 2007; $12: 238-44$

372. Ndao M, Bandyayera E, Kokoskin E, et al. Comparison of blood smear, antigen detection, and nested-PCR methods for screening refugees from regions where malaria is endemic after a malaria outbreak in Quebec, Canada. J Clin Microbiol 2004; 42:2694-700

373. Phares C, Kapella B, Casano A, et al. Imported Plasmodium falciparum malaria disease in newly arrived refugees: United States [poster presentation]. 46th Annual Meeting of Infectious Diseases Society of America; 2008 Oct. 25-28; Washington (DC).

374. Cherian S, Fagan JM, Thambiran A, et al. Severe Plasmodium falciparum malaria in refugee children despite reported predeparture antimalarial treatment. Med J Aust 2006; 185:611

375. Malaria in refugees from Tanzania: King County, Washington, 2007. MMWR Morb Mortal Wkly Rep 2008;57:869-72.

376. Cooke G, Hargreaves S, Natkunarajah J, et al. Impact on and use of an inner-city London Infectious Diseases Department by international migrants: a questionnaire survey. BMC Health Serv Res 2007;7:113.

377. Dias SF, Severo M, Barros H. Determinants of health care utilization by immigrants in Portugal. BMC Health Serv Res 2008;8:207.

378. Lenz K. Migrants in Germany: delay of diagnosis and treatment. J Travel Med 2006;13:133-7

379. Eliades MJ, Shah S, Nguyen-Dinh P, et al. Malaria surveillance - United States, 2003. MMWR Surveill Summ 2005;54(SS02):25-40.

380. Smith AD, Bradley DJ, Smith V, et al. Imported malaria and high risk groups: observational study using UK surveillance data 1987-2006 BMJ 2008;337:a120.

381. Spinazzola F, Nicastri E, Vlassi C, et al. Imported malaria at Italy's National Institute for Infectious Diseases. Eur J Clin Microbiol Infect Dis 2007;26:175-9.

382. Recommendations for pre-departure presumptive treatment and directed treatment for malaria for all refugees from sub Saharan Africa. Atlanta (GA): Centers for Disease Control and Prevention; 2010. Available: www.cdc.gov/immigrantrefugeehealth /guidelines/overseas/malaria-guidelines-overseas.html (accessed 2011 May 5).

383. Patten SB, Kennedy SH, Lam RW, et al. Canadian Network for Mood and Anxiety Treatments (CANMAT) clinical guidelines for the management of major depressive disorder in adults. I. Classification, burden and principles of management. $J$ Affect Disord 2009;117(Suppl 1):S5-14.

384. Bland R. Depression and its management in primary care. Can J Psychiatry 2007; 52:75-6.

385. Kirmayer LJ, Weinfeld M, Burgos G, et al. Use of health care services for psychological distress by immigrants in an urban multicultural milieu. Can J Psychiatry 2007;52:295-304

386. Beiser M. Resettling refugees and safeguarding their mental health: lessons learned from the Canadian Refugee Resettlement Project. Transcult Psychiatry 2009;46: 539-83.

387. Kandula NR, Kersey M, Lurie N. Assuring the health of immigrants: what the leading health indicators tell us. Annu Rev Public Health 2004;25:357-76.

388. MacMillan HL, Patterson CJ, Wathen CN, et al. Screening for depression in primary care: recommendation statement from the Canadian Task Force on Preventive Health Care. CMAJ 2005;172:33-5.

389. U.S. Preventive Services Task Force. Screening for depression: recommendations and rationale. Ann Intern Med 2002;136:760-4.

390. Screening for depression in adults: U.S. Preventive Services Task Force recommendation statement. Ann Intern Med 2009;151:784-92.

391. American Psychiatric Association. Practice guideline for the treatment of patients 
with major depressive disorder (revision). Am J Psychiatry 2000;157(Suppl):1-45.

392. Depression: management of depression in primary and secondary care. Clinical Guideline 23. London (UK): National Institute for Clinical Excellence. ; 2004.

393. Patten SB, Wang JL, Williams JV, et al. Descriptive epidemiology of major depression in Canada. Can J Psychiatry 2006;51:84-90.

394. Ali J. Mental health of Canada's immigrants. Health Rep 2002;13(Suppl):1-11.

395. Stewart DE, Gagnon A, Saucier JF, et al. Postpartum depression symptoms in newcomers. Can J Psychiatry 2008;53:121-4.

396. Zelkowitz P, Schinazi J, Katofsky L, et al. Factors associated with depression in pregnant immigrant women. Transcult Psychiatry 2004;41:445-64.

397. Vesga-Lopez O, Blanco C, Keyes K, et al. Psychiatric disorders in pregnant and postpartum women in the United States. Arch Gen Psychiatry 2008;65:805-15.

398. Gilbody S, Sheldon T, House A. Screening and case-finding instruments for depression: a meta-analysis. CMAJ 2008;178:997-1003.

399. Mitchell AJ, Coyne JC. Do ultrashort screening instruments accurately detect depression in primary care? A pooled analysis and meta-analysis of 22 studies. $\mathrm{Br} \mathrm{J}$ Gen Pract 2007;57:144-51.

400. Pignone MP, Gaynes BN, Rushton JL, et al. Screening for depression in adults: a summary of the evidence for the U.S. Preventive Services Task Force. Ann Intern Med 2002;136:765-76.

401. O'Connor EA, Whitlock EP, Beil TL, et al. Screening for depression in adult patients in primary care settings: a systematic evidence review. Ann Intern Med 2009; 151:793-803.

402. Gilbody S, Bower P, Fletcher J, et al. Collaborative care for depression: a cumulative meta-analysis and review of longer-term outcomes. Arch Intern Med 2006;166: 2314-21.

403. Wells K, Sherbourne C, Schoenbaum M, et al. Five-year impact of quality improvement for depression: results of a group-level randomized controlled trial. Arch Gen Psychiatry 2004;61:378-86.

404. Schraufnagel TJ, Wagner AW, Miranda J, et al. Treating minority patients with depression and anxiety: What does the evidence tell us? Gen Hosp Psychiatry 2006;28:27-36

405. Kirmayer LJ. Cultural variations in the clinical presentation of depression and anxiety: implications for diagnosis and treatment. J Clin Psychiatry 2001;62(Suppl 13): 22-8, discussion 29-30.

406. Kirmayer LJ, Robbins JM, Dworkind M, et al. Somatization and the recognition of depression and anxiety in primary care. Am J Psychiatry 1993;150:734-41.

407. Reproductive mental health guideline 4. Mental illness during the perinatal period: major depression. Vancouver (BC): British Columbia Reproductive Care Program; 2003.

408. McQueen K, Montgomery P, Lappan-Gracon S, et al. Evidence-based recommendations for depressive symptoms in postpartum women. J Obstet Gynecol Neonatal Nurs 2008;37:127-36.

409. ACOG Committee on Health Care for Underserved Women. ACOG Committee Opinion No. 343: psychosocial risk factors: perinatal screening and intervention. Obstet Gynecol 2006;108:469-77.

410. U.S. Preventive Services Task Force. Screening and treatment for major depressive disorder in children and adolescents: US Preventive Services Task Force Recommendation Statement. Pediatrics 2009;123:1223-8

411. Williams SB, O'Connor EA, Eder M, et al. Screening for child and adolescent depression in primary care settings: a systematic evidence review for the US Preventive Services Task Force. Pediatrics 2009;123:e716-35.

412. Minas H, Klimidis S, Ranieri N, et al. Relative prevalence of psychological morbidity in older immigrants. Int J Cult Ment Health 2008;1:58-72.

413. Scheppers E, van Dongen E, Dekker J, et al. Potential barriers to the use of health services among ethnic minorities: a review. Fam Pract 2006;23:325-48.

414. Chen AW, Kazanjian A, Wong H. Why do Chinese Canadians not consult mental health services: health status, language or culture? Transcult Psychiatry 2009;46:623-41.

415. Betancourt JR, Green AR, Carrillo JE, et al. Cultural competence and health care disparities: key perspectives and trends. Health Aff (Millwood) 2005;24:499-505.

416. Goode TD, Dunne MC, Bronheim SM. The evidence base for cultural and linguistic competency in health care. New York (NY): The Commonwealth Fund; 2006.

417. Beach MC, Price EG, Gary TL, et al. Cultural competence: a systematic review of health care provider educational interventions. Med Care 2005;43:356-73.

418. Katon WJ, Seelig M. Population-based care of depression: team care approaches to improving outcomes. J Occup Environ Med 2008;50:459-67.

419. Kates N, Mach M. Chronic disease management for depression in primary care: a summary of the current literature and implications for practice. Can J Psychiatry 2007;52:77-85.

420. Whitley R, Kirmayer L, Groleau D. Understanding immigrants' reluctance to use mental health services: a qualitative study from Montreal. Can J Psychiatry 2006;51:205-9

421. Steel Z, Chey T, Silove D, et al. Association of torture and other potentially traumatic events with mental health outcomes among populations exposed to mass conflict and displacement: a systematic review and meta-analysis. JAMA 2009;302:537-49.

422. National Collaborating Centre for Mental Health, Royal College of Psychiatrists' Research Unit. Post-traumatic stress disorder. The management of PTSD in adults and children in primary and secondary care. National Clinical Practice Guideline no. 26. London (UK): Gaskell and the British Psychological Society; 2005.

423. Ipser J, Carey P, Dhansay Y, et al. Pharmacotherapy augmentation strategies in treatment-resistant anxiety disorders. Cochrane Database Syst Rev 2006;(4):CD005473.
424. Stein DJ, Ipser J, Seedat S. Pharmacotherapy for post traumatic stress disorder (PTSD). Cochrane Database Syst Rev 2006;(1):CD002795.

425. Roberts NP, Kitchiner NJ, Knardy J, et al. Early psychological interventions to treat acute traumatic stress symptoms. Cochrane Database Syst Rev 2010;(3):CD007944.

426. Bisson J, Andrew M. Psychological treatment of post-traumatic stress disorder (PTSD). Cochrane Database Syst Rev 2007;(3):CD003388.

427. Foa EB, Keane TM, Friedman MJ, et al., editors. Effective treatment for PTSD: practice guidelines from the International Society for Traumatic Stress. 2nd ed. New York (NY): The Guilford Press; 2009.

428. Crumlish N, O'Rourke K. A systematic review of treatments for post-traumatic stress disorder among refugees and asylum-seekers. J Nerv Ment Dis 2010;198: 237-51.

429. Lustig SL, Kia-Keating M, Knight WG, et al. Review of child and adolescent refugee mental health. J Am Acad Child Adolesc Psychiatry 2004;43:24-36.

430. Carlson JM. Mental health and health-related quality of life in tortured refugees. Copenhagen (Denmark): University of Copenhagen; 2005.

431. Ursano RJ, Bell C, Eth S, et al. Practice guideline for the treatment of patients with acute stress disorder and posttraumatic stress disorder. Am J Psychiatry 2004;161: 3-31.

432. Beiser M. Strangers at the gate: the "boat people's" first ten years in Canada. Toronto (ON): University of Toronto Press; 1999.

433. Rousseau C, Drapeau A. Are refugee children an at-risk group? A longitudinal study of Cambodian adolescents. J Refug Stud 2003;16:67-81.

434. Rousseau C, Drapeau A. Premigration exposure to political violence among independent immigrants and its association with emotional distress. J Nerv Ment Dis 2004;192:852-6

435. Davis SM, Whitworth JD, Rickett K. What are the most practical primary care screens for post-traumatic stress disorder? J Fam Pract 2009;58:100-1.

436. Prins A, Ouimette P, Kimerling R, et al. The primary care PTSD screen (PC-PTSD): development and operating characteristics. Prim Care Psychiatry 2003;9:9-14.

437. Hackmann A, Ehlers A, Speckens A, et al. Characteristics and content of intrusive memories of PTSD and the changes with treatment. J Trauma Stress 2004;17:231-40.

438. Moreno A, Grodin MA. Torture and its neurological sequelae. Spinal Cord 2002; 40:213-23.

439. Guidelines for mental health screening during the domestic medical examination for newly arrived refugees. Atlanta (GA): Centers for Disease Control and Prevention; 2011. Available: www.cdc.gov/immigrantrefugeehealth/guidelines/domestic/mentalhealth-screening-guidelines.html (accessed 2011 July 1).

440. Prévention de la violence. In: Cinquantième Assemblée mondiale de la santé; 1997 May 5-14; Geneva. Available: www.who.int/violence_injury_prevention/resources /publications/en/WHA5019_fre.pdf

441. Trocmé N, Fallon B, MacLaurin B, et al. Canadian incidence study of reported child abuse and neglect - 2003: major findings. Ottawa (ON): Minister of Public Works and Government Services Canada; 2005.

442. Wyatt GE, Peters SD. Issues in the definition of child sexual abuse in prevalence research. Child Abuse Negl 1986;10:231-40.

443. Nygren P, Nelson HD, Klein J. Screening children for family violence: a review of the evidence for the US Preventive Services Task Force. Ann Fam Med 2004;2:161-9.

444. MacMillan HL; Canadian Task Force on Preventive Health Care. Preventive health care, 2000 update: prevention of child maltreatment. CMAJ 2000;163:1451-8.

445. Peters R, Barlow J. Systematic review of instruments designed to predict child maltreatment during the antenatal and postnatal periods. Child Abuse Rev 2003;12:416-39.

446. Barlow J, Johnston I, Kendrick D, et al. Individual and group-based parenting programmes for the treatment of physical child abuse and neglect. Cochrane Database Syst Rev 2006;(3):CD005463.

447. Macdonald G, Higgins JPT, Ramchandani P. Cognitive-behavioural interventions for children who have been sexually abused. Cochrane Database Syst Rev 2006; (4):CD001930

448. Hill RB. Synthesis of research on disproportionality in child welfare: an update Seattle (WA): Casey-CSSP Alliance for Racial Equity; 2006

449. Trocmé N, Knoke D, Fallon B, et al. CIS-2003: Understanding the case substantiation decision. Ottawa (ON): Public Health Agency of Canada; 2006.

450. Elliott K, Urquiza A. Ethnicity, culture, and child maltreatment. J Soc Issues 2006; 62:787-809.

451. Dezerotes DM, Poertner J, Testa MF, editors. Race matters in child welfare: the overrepresentation of African Americans in the system. Washington (DC): Child Welfare League of America Press; 2005.

452. Eckenrode J, Powers J, Doris J, et al. Substantiation of child abuse and neglect reports. J Consult Clin Psychol 1988;56:9-16.

453. Sedlak A, Schultz D. Racial differences in child protective services investigation of abused and neglected children. In: Derezotes D, Testa MF, Poertner J, editors. Race matters in child welfare: the overrepresentation of African Americans in the system. Washington (DC): Child Welfare League of America Press; 2005. p. 97-118.

454. Terao SY, Borrego J Jr, Urquiza AJ. A reporting and response model for culture and child maltreatment. Child Maltreat 2001;6:158-68.

455. Zellman GL. The impact of case characteristics on child abuse reporting decisions. Child Abuse Negl 1992;16:57-74.

456. U.S. Preventive Service Task Force. Screening for family and intimate partner violence: recommendation statement. Ann Intern Med 2004;140:382-6.

457. Woodman J, Pitt M, Wentz R, et al. Performance of screening tests for child physical abuse in accident and emergency departments. Health Technol Assess 2008;12:iii, xi-xiii, 1-95. 
458. Morh WK, Tulman LJ. Children exposed to violence: measurement considerations within an ecological framework. ANS Adv Nurs Sci 2000;23:59-68.

459. Olds DL, Eckenrode J, Henderson CR Jr, et al. Long-term effects of home visitation on maternal life course and child abuse and neglect: fifteen-year follow-up of a randomized trial. JAMA 1997;278:637-43.

460. Mikton C, Butchart A. Child maltreatment prevention: a systematic review of reviews. Bull World Health Organ 2009;87:353-61.

461. Olds D, Henderson C, Kitzman H, et al. Effects of prenatal and infancy nurse home visitation on surveillance of child maltreatment. Pediatrics 1995;95:365-72.

462. Kitzman H, Olds DL, Henderson CR Jr, et al. Effect of prenatal and infancy home visitation by nurses on pregnancy outcomes, childhood injuries, and repeated childbearing: a randomized controlled trial. JAMA 1997;278:644-52.

463. Macmillan HL, Wathen CN, Barlow J, et al. Interventions to prevent child maltreatment and associated impairment. Lancet 2009;373:250-66.

464. Cohen JA, Mannarino AP, Murray LK, et al. Psychosocial interventions for maltreated and violence-exposed children. J Soc Issues 2006;62:737-66.

465. Chaffin M, Silovsky JF, Funderburk B, et al. Parent-child interaction therapy with physically abusive parents: efficacy for reducing future abuse reports. $J$ Consult Clin Psychol 2004:72:500-10.

466. Larrivée MC, Tourigny M, Bouchard C. Child physical abuse with and without other forms of maltreatment: dysfunctionality versus dysnormality. Child Maltreat 2007;12:303-13.

467. Dubowitz H, Feigelman S, Lane W, et al. Pediatric primary care to help prevent child maltreatment: the Safe Environment for Every Kid (SEEK) model. Pediatrics 2009; 123:858-64.

468. American Academy of Pediatrics, Committee on Child Abuse and Neglect. Guidelines for the evaluation of sexual abuse of children. Pediatrics 1991;87:254-60

469. American Medical Association. Diagnostic and treatment guidelines on child sexual abuse. Arch Fam Med 1993;2:19-27.

470. American Medical Association. Diagnostic and treatment guidelines on child physical abuse and neglect. Arch Fam Med 1992;1:187-97.

471. Violence prevention focused on children and youth: early childhood home visitation. In: Guide to community preventive services. Atlanta (GA): Centers for Disease Control and Prevention; 2010. Available: www.thecommunityguide.org/violence /home/index.html (accessed 2011 May 5).

472. Ramsay J, Carter Y, Davidson L, et al. Advocacy interventions to reduce or eliminate violence and promote the physical and psychosocial well-being of women who experience intimate partner abuse. Cochrane Database Syst Rev 2009; (3):CD005043

473. Canadian Centre for Justice Statistics. A profile of criminal victimization: results of the 1999 General Social Survey. Ottawa (ON): The Centre; 2000. Cat. no. 85-553-XIE. Available: www.statcan.gc.ca/pub/85-553-x/85-553-x1999001-eng.pdf (accessed 2011 May 6)

474. Family violence in Canada: a statistical profile. Ottawa (ON): Statistics Canada; 2002. Cat no. 85-224-XWE

475. Wathen CN, Macmillan HL. Interventions for violence against women: scientific review. JAMA 2003;289:589-600.

476. Nelson HD, Nygren P, McInerney Y. Screening for family and intimate partner violence: systematic evidence review. Rockville (MD): US Preventive Services Task Force; 2004.

477. Feder G, Ramsay J, Dunne D, et al. How far does screening women for domestic (partner) violence in different health-care settings meet criteria for a screening programme? Systematic reviews of nine UK National Screening Committee criteria. Health Technol Assess 2009; 13:iii-iv, xi-xiii, 1-113, 137-347.

478. Macmillan HL, Wathen CN, Jamieson E, et al. Screening for intimate partner violence in health care settings: a randomized trial. JAMA 2009;302:493-501.

479. Rabin RF, Jennings JM, Campbell JC, et al. Intimate partner violence screening tools: a systematic review. Am J Prev Med 2009;36:439-45.

480. Brownridge DA, Halli SS. Double jeopardy: violence against immigrant women in Canada. Violence Vict 2002;17:455-71.

481. Hyman I, Forte T, Du Mont J, et al. The association between length of stay in Canada and intimate partner violence among immigrant women. Am J Public Health 2006;96:654-9.

482. Ahmad F, Ali M, Stewart DE. Spousal-abuse among Canadian immigrant women. J Immigr Health 2005;7:239-46.

483. Macmillan HL, Wathen CN, Jamieson E. Approaches to screening for intimate partner violence in health care settings: a randomized trial. JAMA 2006;296:530-6.

484. Ahmad F, Hogg-Johnson S, Stewart DE, et al. Computer-assisted screening for intimate partner violence and control: a randomized trial. Ann Intern Med 2009; 151:93-102.

485. Cherniak D, Grant L, Mason R, et al. Intimate partner violence consensus statement. J Obstet Gynaecol Can. 2005;27:365-388.

486. Taket A, Wathen CN, Macmillan H. Should health professionals screen all women for domestic violence? PLoS Med 2004; e4. [Epub 2004 Oct 19].

487. Macmillan HL, Wathen CN. Prevention and treatment of violence against women: systematic review and recommendations. London $(\mathrm{ON})$ : Canadian Task Force on Preventive Health Care; 2001.

488. Glass N, Dearwater S, Campbell J. Intimate partner violence screening and intervention: data from eleven Pennsylvania and California community hospital emergency departments. J Emerg Nurs 2001;27:141-9.

489. Furbee PM, Sikora R, Williams JM, et al. Comparison of domestic violence screening methods: a pilot study. Ann Emerg Med 1998;31:495-501.

490. Chang JC, Decker M, Moracco KE, et al. What happens when health care providers ask about intimate partner violence? A description of consequences from the perspectives of female survivors. J Am Med Womens Assoc 2003;58:76-81.

491. Sullivan CM, Bybee DI. Reducing violence using community-based advocacy for women with abusive partners. J Consult Clin Psychol 1999;67:43-53.

492. Sullivan CM. Using the ESID model to reduce intimate male violence against women. Am J Community Psychol 2003:32:295-303.

493. Sullivan CM, Tan C, Basta J, et al. An advocacy intervention program for women with abusive partners: initial evaluation. Am J Community Psychol 1992;20:309-32.

494. Hyman I, Forte T, Du Mont J, et al. Help-seeking rates for intimate partner violence (IPV) among Canadian immigrant women. Health Care Women Int 2006;27:682-94.

495. Dutton MA, Orloff LE, Aguilar Hass G. Characteristics of help-seeking behaviors, resources and service needs of battered immigrant latinas: legal and policy implications. Georgetown J Poverty Law Policy 2000;7:245-305.

496. Bhuyan R, Senturia K. Understanding domestic violence resource utilization and survivor solutions among immigrant and refugee women: introduction to the special issue. J Interpers Violence 2005;20:895-901.

497. Crandall M, Senturia K, Sullivan M, et al. "No way out": Russian-speaking women's experiences with domestic violence. J Interpers Violence 2005;20:941-58.

498. Sullivan M, Senturia K, Negash T, et al. "For us it is like living in the dark": Ethiopian women's experiences with domestic violence. J Interpers Violence 2005; 20:922-40.

499. Diabetes report 2005. Assessing provincial, territorial and federal government policies and programs for Canadians with diabetes. Ottawa (ON): Canadian Diabetes Association; 2009. Available: www.diabetes.ca/files/diabetesreport2005 /CDA-diabetesreport-2005-en.pdf (accessed 2009 Oct. 27).

500. Carter JS, Pugh JA, Monterrosa A. Non-insulin-dependent diabetes mellitus in minorities in the United States. Ann Intern Med 1996;125:221-32.

501. U.S. Preventive Services Task Force. Screening for type 2 diabetes mellitus in adults: U.S. Preventive Services Task Force recommendation statement. Ann Intern Med 2008;148:846-54

502. Cauchi S, ElAchhab Y, Choquet H, et al. TCF7L2 is reproducibly associated with type 2 diabetes in various ethnic groups: a global meta-analysis. J Mol Med 2007; $85: 777-82$

503. Kunz R, Bork JP, Fritsche L, et al. Association between the angiotensin-converting enzyme-insertion/deletion polymorphism and diabetic nephropathy: a methodologic appraisal and systematic review. J Am Soc Nephrol 1998;9:1653-63.

504. Ludovico O, Pellegrini F, Di Paola R, et al. Heterogeneous effect of peroxisome proliferator-activated receptor gamma2 Ala12 variant on type 2 diabetes risk. Obesity (Silver Spring) 2007;15:1076-81

505. Peek ME, Cargill A, Huang ES. Diabetes health disparities: a systematic review of health care interventions. Med Care Res Rev 2007;64(5 Suppl):101S-56S

506. Kirk JK, D'Agostino RB Jr, Bell RA, et al. Disparities in HbA1c levels between African-American and non-Hispanic white adults with diabetes: a meta-analysis. Diabetes Care 2006;29:2130-6.

507. Caban A, Walker EA. A systematic review of research on culturally relevant issues for Hispanics with diabetes. Diabetes Educ 2006;32:584-95.

508. Whittemore R. Culturally competent interventions for Hispanic adults with type 2 diabetes: a systematic review. J Transcult Nurs 2007;18:157-66.

509. Canadian Diabetes Association Clinical Practice Guidelines Expert Committee. Canadian Diabetes Association 2008 clinical practice guidelines for the prevention and management of diabetes in Canada. Can J Diabetes 2008;32(Suppl 1):S1-201.

510. US Preventive Services Task Force. Screening for type 2 diabetes mellitus in adults: recommendations and rationale. Ann Intern Med 2003;138:212-4.

511. Ray KK, Seshasai SR, Wijesuriya S, et al. Effect of intensive control of glucose on cardiovascular outcomes and death in patients with diabetes mellitus: a metaanalysis of randomised controlled trials. Lancet 2009;373:1765-72.

512. Kandula NR, Diez-Roux AV, Chan C, et al. Association of acculturation levels and prevalence of diabetes in the multi-ethnic study of atherosclerosis (MESA). Diabetes Care 2008;31:1621-8.

513. Davis TM, Cull CA, Holman RR. Relationship between ethnicity and glycemic control, lipid profiles, and blood pressure during the first 9 years of type 2 diabetes: U.K. Prospective Diabetes Study (UKPDS 55). Diabetes Care 2001;24:1167-74.

514. Koopman RJ, Mainous AG 3rd, Diaz VA, et al. Changes in age at diagnosis of type 2 diabetes mellitus in the United States, 1988 to 2000. Ann Fam Med 2005;3:60-3.

515. Ramachandran A, Snehalatha C, Latha E, et al. Rising prevalence of NIDDM in an urban population in India. Diabetologia 1997;40:232-7.

516. Vazquez G, Duval S, Jacobs DR Jr, et al. Comparison of body mass index, waist circumference, and waist/hip ratio in predicting incident diabetes: a meta-analysis. Epidemiol Rev 2007;29:115-28.

517. Sargeant LA, Bennett FI, Forrester TE, et al. Predicting incident diabetes in Jamaica: the role of anthropometry. Obes Res 2002;10:792-8.

518. Clinical Guidelines Taskforce. Global guideline for type 2 diabetes. Brussels (Belgium): International Diabetes Federation; 2005. Available: www.idf.org/webdata /docs/IDF\%20GGT2D.pdf (accessed 2011 May 6).

519. Alberti KGMM, Zimmet P, Shaw J. Metabolic syndrome: a new worldwide definition. A consensus statement from the International Diabetes Federation. Diabe Med 2006;23:469-80.

520. Bennett CM, Guo M, Dharmage SC. $\mathrm{HbA}(1 \mathrm{c})$ as a screening tool for detection of type 2 diabetes: a systematic review. Diabet Med 2007;24:333-43.

521. Harris R, Donahue K, Rathore SS, et al. Screening adults for type 2 diabetes: a review of the evidence for the U.S. Preventive Services Task Force. Ann Intern Med 2003;138:215-29.

522. Turnbull F, Neal B, Algert C, et al. Effects of different blood pressure-lowering 
regimens on major cardiovascular events in individuals with and without diabetes mellitus: results of prospectively designed overviews of randomized trials. Arch Intern Med 2005;165:1410-9.

523. Orozco LJ, Buchleitner AM, Gimenez-Perez G, et al. Exercise or exercise and diet for preventing type 2 diabetes mellitus. Cochrane Database Syst Rev 2008;(3): CD003054.

524. Glazier RH, Bajcar J, Kennie NR, et al. A systematic review of interventions to improve diabetes care in socially disadvantaged populations. Diabetes Care 2006;29:1675-88

525. Norris SL, Kansagara D, Bougatsos C, et al. Screening adults for type 2 diabetes: a review of the evidence for the U.S. Preventive Services Task Force. Ann Intern Med 2008;148:855-68

526. American Diabetes Association. Standards of medical care in diabetes - 2010. Diabetes Care 2010;33(Suppl 1):S11-S61.

527. Recommendations to prevent and control iron deficiency in the United States. MMWR Recomm Rep 1998;47:1-29.

528. Sachdev H, Gera T, Nestel P. Effect of iron supplementation on physical growth in children: systematic review of randomized controlled trials. Public Health Nutr 2006;9:904-20

529. Sachdev H, Gera T, Nestel P. Effect of iron supplementation on mental and motor development in children: systematic review of randomised controlled trials. Public Health Nutr 2005;8:117-32.

530. Li R, Chen X, Yan H, et al. Functional consequences of iron supplementation in iron-deficient female cotton mill workers in Beijing, China. Am J Clin Nutr 1994; 59:908-13.

531. U.S. Preventive Services Task Force. Screening for iron deficiency anemia including iron supplementation for children and pregnant women: recommendation statement. Rockville (MD): Agency for Healthcare Research and Quality; 2006. Available: www.ahrq.gov/clinic/uspstf06/ironsc/ironrs.htm (accessed 2008 June 30).

532. Murray-Kolb LE, Beard JL. Iron treatment normalizes cognitive functioning in young women. Am J Clin Nutr 2007;85:778-87.

533. Gera T, Sachdev HP. Effect of iron supplementation on incidence of infectious illness in children: systematic review. BMJ 2002;325:1-10.

534. Hassan K, Sullivan KM, Yip R, et al. Factors associated with anemia in refugee children. J Nutr 1997;127:2194-8.

535. Brotanek JM, Gosz J, Weitzman M, et al. Iron deficiency in early childhood in the United States: risk factors and racial/ethnic disparities. Pediatrics 2007;120:568-75.

536. López-Vélez R, Huerga H, Turrientes MC. Infectious disease in immigrants from the perspective of a tropical medicine referral unit. Am J Trop Med Hyg 2003;69: 115-21

537. Pena-Rosas JP, Viteri FE. Effects of routine oral iron supplementation with or without folic acid for women during pregnancy. Cochrane Database Syst Rev 2007;(3):CD004736

538. Reveiz GL, Cuervo LG. Treatments for iron-deficiency anaemia in pregnancy. Cochrane Database Syst Rev 2007;(1):CD003094.

539. Sloan NL, Jordan E, Winikoff B. Effects of iron supplementation on maternal hematologic status in pregnancy. Am J Public Health 2002;92:288-93.

540. Feightner JW. Prevention of iron deficiency anemia in infants. In: Canadian Task Force on the Periodic Health Examination. Canadian guide to clinical preventive health care. Ottawa (ON): Health Canada; 1994. p. 244-55.

541. Basta SS, Soekirman, Karyadi D, et al. Iron deficiency anemia and the productivity of adult males in Indonesia. Am J Clin Nutr 1979;32:916-25.

542. Edgerton VR, Gardner GW, Ohira Y, et al. Iron-deficiency anaemia and its effect on worker productivity and activity patterns. BMJ 1979;2:1546-9.

543. Menon P, Ruel MT, Loechl CU, et al. Micronutrient sprinkles reduce anemia among 9- to 2-mo-old children when delivered through an Integrated Health and Nutrition Program in rural Haiti. J Nutr 2007;137:1023-30.

544. Anderson J, Kirkham RS. Constructing nation: the gendering and racialization of the Canadian health care system. In: Strong-Boag V, Grace S, Eisenberg A, et al., editors. Painting the maple: essays on race, gender and the constructions of Canada. Vancouver (BC): UBC Press; 1998. p. 242-61.

545. World Health Organization. Iron deficiency anaemia: assessment, prevention and control. A guide for programme managers. Geneva (CH): The Organization; 2001. Available: www.who.int/nutrition/publications/micronutrients/anaemia_iron deficiency/WHO_NHD_01.3/en/index.html (accessed 2009 Nov. 1).

546. Petersen PE, Bourgeois D, Ogawa H, et al. The global burden of oral diseases and risks to oral health. Bull World Health Organ 2005;83:661-9.

547. Kay E, Locker D. A systematic review of the effectiveness of health promotion aimed at improving oral health. Community Dent Health 1998;15:132-44.

548. Sutherland S, Matthews DC. Emergency management of acute apical periodontitis in the permanent dentition: a systematic review of the literature. J Can Dent Assoc 2003;69:160. [to be incorporated into numbering scheme]

549. Fedorowicz Z, Keenan JV, Farman AG, et al. Antibiotic use for irreversible pulpitis. Cochrane Database Syst Rev 2005;(2):CD004969.

550. Azarpazhooh A, Main PA. Fluoride varnish in the prevention of dental caries in children and adolescents: a systematic review. J Can Dent Assoc 2008;74:73-9.

551. Marinho VC, Higgins JP, Sheiham A, et al. Combinations of topical fluoride (toothpastes, mouthrinses, gels, varnishes) versus single topical fluoride for preventing dental caries in children and adolescents. Cochrane Database Syst Rev 2004;(1):CD002781

552. Marinho VC, Higgins JP, Sheiham A, et al. One topical fluoride (toothpastes, or mouthrinses, or gels, or varnishes) versus another for preventing dental caries in children and adolescents. Cochrane Database Syst Rev 2004;(1):CD002780.
553. Marinho VC, Higgins JP, Logan S, et al. Fluoride mouthrinses for preventing dental caries in children and adolescents. Cochrane Database Syst Rev 2003;(3): CD002284.

554. Marinho VCC. Topical fluoride (toothpastes, mouthrinses, gels or varnishes) for preventing dental caries in children and adolescents. Cochrane Database Syst Rev 2003;(4):CD002782.

555. Ahovuo-Saloranta A, Hiiri A, Nordblad A, et al. Pit and fissure sealants for preventing dental decay in the permanent teeth of children and adolescents. Cochrane Database Syst Rev 2004;(4):CD001830.

556. Hiiri A, Ahovuo-Saloranta A, Nordblad A, et al. Pit and fissure sealants versus fluoride varnishes for preventing dental decay in children and adolescents. Cochrane Database Syst Rev 2010;(3):CD003067.

557. Griffin SO, Oong E, Kohn W, et al.; CDC Dental Sealant Systematic Review Work Group. The effectiveness of sealants in managing caries lesions. J Dent Res 2008;87:169-74

558. Griffin SO, Regnier E, Griffin PM, et al. Effectiveness of fluoride in preventing caries in adults. $J$ Dent Res 2007;86:410-5.

559. Ricketts DN, Kidd EA, Innes N, et al. Complete or ultraconservative removal of decayed tissue in unfilled teeth. Cochrane Database Syst Rev 2006;(3):CD003808.

560. Locker D, Clarke M, Murray H. Oral health status of Canadian-born and immigrant adolescents in North York, Ontario. Community Dent Oral Epidemiol 1998;26:177-81.

561. Full report: Emergency management of acute apical periodontitis in adults. Canadian Collaboration on Clinical Practice Guidelines in Dentistry; 2003. Available: www.cda-adc.ca/_files/dental_profession/practising/clinical_practice_guidelines/FULL _VERSION.pdf (accessed 2010 Nov. 5).

562. Barden J, Edwards JE, McQuay HJ, et al. Relative efficacy of oral analgesics after third molar extraction. Br Dent J 2004;197:407-11; discussion 397.

563. Roelofs PD, Deyo RA, Koes BW, et al. Non-steroidal anti-inflammatory drugs for low back pain. Cochrane Database Syst Rev 2008;(1):CD000396.

564. Bedos C, Brodeur J, Benigeri M, et al. [Utilization of preventive dental services by recent immigrants in Quebec]. Can J Public Health 2004;95:219-23. French.

565. Cruz GD, Shore R, Le Geros RZ, et al. Effect of acculturation on objective measures of oral health in Haitian immigrants in New York City. J Dent Res 2004;83: $180-4$

566. Ma M, Lindsell CJ, Jauch EC, et al. Effect of education and guidelines for treatment of uncomplicated dental pain on patient and provider behavior. Ann Emerg Med 2004;44:323-9.

567. Lewis CW, Grossman DC, Domoto PK, et al. The role of the pediatrician in the oral health of children: a national survey. Pediatrics 2000;106:E84.

568. Lawrence HP, Binguis D, Douglas J, et al. A 2-year community-randomized controlled trial of fluoride varnish to prevent early childhood caries in aboriginal children. Community Dent Oral Epidemiol 2008;36:503-16.

569. Scottish Intercollegiate Guidelines Network. SIGN 47: Preventing dental caries in children at high caries risk targeted prevention of dental caries in the permanent teeth of 6-16 year olds presenting for dental care. Edinburgh (UK): The Network; 2000. Available: www.sign.ac.uk/guidelines/fulltext/47/index.html (accessed 2010 Nov. 5).

570. Scottish Intercollegiate Guidelines Network. SIGN 83: Prevention and manage ment of dental decay in the pre-school child. Edinburgh (UK): The Network; 2005. Available: www.sign.ac.uk/pdf/sign83.pdf (accessed 2010 Nov. 5).

571. Chiang PP, Keeffe JE, Le Mesurier RT, et al. Global burden of disease and visual impairment. Lancet 2006;368:365.

572. Lopez AD, Mathers CD, Ezzati M, et al. Global and regional burden of disease and risk factors, 2001: systematic analysis of population health data. Lancet 2006;367: 1747-57.

573. West SK, Munoz B, Rubin GS, et al. Function and visual impairment in a population-based study of older adults. The SEE project. Salisbury Eye Evaluation. Invest Ophthalmol Vis Sci 1997;38:72-82.

574. Rovner BW, Ganguli M. Depression and disability associated with impaired vision: the MoVies Project. J Am Geriatr Soc 1998;46:617-9.

575. Klein BEK, Klein R, Lee KE, et al. Performance-based and self-assessed measures of visual function as related to history of falls, hip fractures, and measured gait time. Ophthalmology 1998;105:160-4.

576. Dandona L, Dandona R. What is the global burden of visual impairment? BMC Med 2006;4:6.

577. Vedula SS, Krzystolik MG. Antiangiogenic therapy with anti-vascular endothelial growth factor modalities for neovascular age-related macular degeneration. Cochrane Database Syst Rev 2008;(2):CD005139.

578. Age-Related Eye Disease Study Research Group. A randomized, placebocontrolled, clinical trial of high-dose supplementation with vitamins $\mathrm{C}$ and $\mathrm{E}$, beta carotene, and zinc for age-related macular degeneration and vision loss: AREDS report No. 8. Arch Ophthalmol 2001;119:1417-36.

579. Taylor HR, Keeffe JE. World blindness: a 21 st century perspective. Br J Ophthalmol 2001;85:261-6.

580. Smeeth L, Iliffe S. Community screening for visual impairment in the elderly. Cochrane Database Syst Rev 2006;(3):001054.

581. Quigley HA, Broman AT. The number of people with glaucoma worldwide in 2010 and 2020. Br J Ophthalmol 2006;90:262-7.

582. Varma R, Wang MY, Ying-Lai M, et al. The prevalence and risk indicators of uncorrected refractive error and unmet refractive need in Latinos: the Los Angeles Latino Eye Study. Invest Ophthalmol Vis Sci 2008;49:5264-73.

583. Varma R, Mohanty SA, Deneen J, et al. Burden and predictors of undetected eye disease in Mexican-Americans: the Los Angeles Latino Eye Study. Med Care 
2008;46:497-506

584. Wilson MR, Eezzuduemhoi DR. Ophthalmologic disorders in minority populations. Med Clin North Am 2005;89:795-804.

585. Munoz B, West SK, Rodriguez J, et al. Blindness, visual impairment and the problem of uncorrected refractive error in a Mexican-American population: Proyecto VER. Invest Ophthalmol Vis Sci 2002;43:608-14

586. Tielsch JM, Sommer A, Katz J, et al. Racial variations in the prevalence of primary open-angle glaucoma. The Baltimore Eye Survey. JAMA 1991;266:369-74.

587. Harris MI, Klein R, Cowie CC, et al. Is the risk of diabetic retinopathy greater in non-Hispanic blacks and Mexican Americans than in non-Hispanic whites with type 2 diabetes? A U.S. population study. Diabetes Care 1998;21:1230-5.

588. Sommer A, Tielsch JM, Katz J, et al. Racial differences in the cause-specific prevalence of blindness in east Baltimore. N Engl J Med 1991;325:1412-7.

589. Vitale S, Cotch MF, Sperduto RD. Prevalence of visual impairment in the United States. JAMA 2006;295:2158-63

590. Ederer F, Krueger DE, Mowery RL, et al. Lessons from the Visual Acuity Impairment Survey pilot study. Am J Public Health 1986;76:160-5.

591. Ferris FL 3rd, Kassoff A, Bresnick GH, et al. New visual acuity charts for clinical research. Am J Ophthalmol 1982;94:91-6.

592. Loewenstein JI, Palmberg PF, Connett JE, et al. Effectiveness of a pinhole method for visual acuity screening. Arch Ophthalmol 1985;103:222-3.

593. Williams C, Harrad RA, Harvey I, et al. Screening for amblyopia in preschool children: results of a population-based, randomised controlled trial. ALSPAC Study Team. Avon Longitudinal Study of Pregnancy and Childhood. Ophthalmic Epidemiol 2001;8:279-95.

594. Foundations for a Canadian Vision Health Strategy: towards preventing avoidable blindness and promoting vision health. Toronto $(\mathrm{ON})$ : National Coalition for Vision Health; 2007.

595. Lewallen S, Courtright P. Gender and use of cataract surgical services in developing countries. Bull World Health Organ 2002;80:300-3.

596. Holden BA, Sulaiman S, Knox K. The challenge of providing spectacles in the developing world. Community Eye Health 2000;13:9-10.

597. Feldman W, Fletcher AE. Well-baby care in the first two years of life. In: Goldbloom R, editor. The Canadian Task Force on the Periodic Health Examination. The Canadian guide to clinical preventive health care. Ottawa (ON): Health Canada; 1994. p. 258-66.

598. Feightner JW. Routine preschool screening for visual and hearing problems. In: Goldbloom R, editor. The Canadian Task Force on the Periodic Health Examination. The Canadian guide to clinical preventive health care. Ottawa $(\mathrm{ON})$ : Health Canada; 1994. p. 298-304

599. Eye examination in infants, children, and young adults by pediatricians. Pediatrics 2003;111(4 pt 1):902-7.

600. Canadian Task Force on the Periodic Health Examination. Periodic health examination, 1995 update: 3 . Screening for visual problems among elderly patients. CMAJ 1995; 152:1211-22.

601. Blustein JFD. Screening for visual impairment. In: Digiseppi C, Atkins D, Woolfe $\mathrm{S}$, et al., editors. Guide to clinical preventive services-report of the U.S. Preventive Services Task Force. 2nd ed. Alexandria (VA): International Medical Publishing; 1996. p. 373-82.

602. Dandona L, Dandona R. Revision of visual impairment definitions in the International Statistical Classification of Diseases. BMC Med 2006:4:7.

603. Atkins D, Patterson C. Screening for glaucoma. In: Digiseppi C, Atkins D, Woolfe $\mathrm{S}$, et al., editors. Guide to clinical preventive services-report of the U.S. Preventive Services Task Force. 2nd ed. Alexandria (VA): International Medical Publishing; 1996. p. 383-91.

604. Canadian Diabetes Association Clinical Practice Guidelines Expert Committee. Canadian Diabetes Association 2003 clinical practice guidelines for the prevention and management of diabetes in Canada. Can J Diabetes 2003;27:S76-80.

605. Programme of action of the United Nations International Conference on Population and Development (ICPD); 1994 Sept. 5-13; Cairo (EG). New York (NY): United Nations Department of Public Information; 1995.

606. Glasier A, Gulmezoglu AM, Schmid GP, et al. Sexual and reproductive health: a matter of life and death. Lancet 2006;368:1595-607.

607. Ross JA, Winfrey WL. Unmet need for contraception in the developing world and former Soviet Union: an updated estimate. Int Fam Plan Perspect 2002;28:138-43.

608. Sedgh G, Hussain R, Bankole A, et al. Women with an unmet need for contraception in developing countries and their reasons for not using a method. New York (NY) and Washington (DC): Guttmacher Institute; 2007. Available: www.policyarchive .org/handle/10207/5945 (accessed 2010 Mar. 9)

609. Moos MK, Bartholomew NE, Lohr KN. Counseling in the clinical setting to prevent unintended pregnancy: an evidence-based research agenda. Contraception 2003;67:115-32.

610. Paine K, Thorogood M, Wellings K. The impact of the quality of family planning services on safe and effective contraceptive use: a systematic literature review. Hum Fertil (Camb) 2000;3:186-93.

611. Becker D, Koenig MA, Kim YM, et al. The quality of family planning services in the United States: findings from a literature review. Perspect Sex Reprod Health 2007:39:206-15

612. U.S. Preventive Services Task Force. Counseling to prevent unintended pregnancy. In: Guide to clinical preventive services. 2nd ed. Rockville (MD): Agency for Healthcare Research and Quality; 1996. p. 739-53.

613. One to one interventions to reduce the transmission of sexually transmitted infections (STIs) including HIV, and to reduce the rate of under 18 conceptions, espe- cially among vulnerable and at risk groups. NICE Public Health Intervention Guidance 3. London (UK): National Institute for Health and Clinical Excellence; 2007. Available: www.nice.org.uk/PHI003 (accessed 2010 Mar. 9).

614. Canto De Cetina TE, Canto P, Ordoñez Luna M. Effect of counseling to improve compliance in Mexican women receiving depot-medroxyprogesterone acetate Contraception 2001:63:143-6.

615. Shlay JC, Mayhugh B, Foster M, et al. Initiating contraception in sexually transmitted disease clinic setting: a randomized trial. Am J Obstet Gynecol 2003;189:473-81.

616. Winter L, Breckenmaker LC. Tailoring family planning services to the specia needs of adolescents. Fam Plann Perspect 1991;23:24-30.

617. Nawar L, Kharboush I, Ibrahim MA, et al. Impact of improved client-provide interaction on women's achievement of fertility goals in Egypt. FRONTIERS final report. Washington (DC): Population Council; 2004. Available: www.popcounci .org/pdfs/frontiers/FR_FinalReports/Egypt_CPI.pdf (accessed 2010 Mar. 9).

618. Mendez F, Lopez F, Brambila C, et al. Screening family planning needs: an operations research project in Guatemala. BMC Int Health Hum Rights 2004;4:2.

619. Foreit JR, Vernon R, Hamel PR. Use of systematic screening to increase the provision of reproductive health services in Bolivia. FRONTIERS final report. Washington (DC): Population Council; 2005. Available: www.popcouncil.org/pdfs/frontiers /FR_FinalReports/Bolivia_Screen.pdf (accessed 2010 Mar. 9)

620. Lindstrom DP, Hernandez CH. Internal migration and contraceptive knowledge and use in Guatemala. Int Fam Plan Perspect 2006;32:146-53.

621. Wolff H, Stalder H, Epiney M, et al. Health care and illegality: a survey of undocumented pregnant immigrants in Geneva. Soc Sci Med 2005;60:2149-54.

622. Jones RK, Finer LB, Singh S. Characteristics of US abortion patients, 2008. New York (NY): Guttmacher Institute, 2010.

623. Moreau C, Bouyer J, Gilbert F, et al. Social demographic and situation characteristics associated with inconsistent use of oral contraceptive; evidence from France. Perspect Sex Reprod Health 2006;38:190-6.

624. Steiner MJ, Dalebout S, Condom S, et al. Understanding risk: a randomized controlled trial of communicating contraceptive effectiveness. Obstet Gynecol 2003:102:709-17.

625. Medical eligibility criteria for contraceptive use. 3rd ed. Geneva $(\mathrm{CH})$ : World Health Organization; 2004. Available: http://whqlibdoc.who.int/publications /2004/9241562668.pdf (accessed 2010 Mar. 9).

626. World contraceptive use 2007. New York (NY): United Nations, Department of Economic and Social Affairs, Population Division; 2007. Available: www.un.org/esa /population/publications/contraceptive2007/contraceptive_2007_table.pdf (accessed 2009 Aug. 24)

627. Gagnon A, Merry L, Robinson C. A systematic review of refugee women's reproductive health. Refuge Can Period Refug 2002;21:6-17.

628. Busza J, Lush L. Planning reproductive health in conflict: a conceptual framework. Soc Sci Med 1999;49:155-71.

629. Stephenson R, Baschieri A, Clements S, et al. Contextual influences on modern contraceptive use in sub-Saharan Africa. Am J Public Health 2007;97:1233-40.

630. Fisher W, Boroditsky R, Morris B. The 2002 Canadian contraception study: part 1. J Obstet Gynaecol Can 2004;26:580-90.

631. Martin K, Wu Z. Contraceptive use in Canada: 1984-1995. Fam Plann Perspect 2000;32:65-73.

632. Sedgh G, Henshaw SK, Singh S, et al. Legal abortion worldwide: incidence and recent trends. Int Fam Plan Perspect 2007;33:106-16.

633. Legardy JK, Curtis KM. Progestogen-only contraceptive use among women with sickle cell anemia: a systematic review. Contraception 2006;73:195-204.

634. Manchikanti A, Grimes DA, Lopez LM, et al. Steroid hormones for contraception in women with sickle cell disease. Cochrane Database Syst Rev 2007;(2):CD006261.

635. Cleland J, Bernstein S, Ezeh A, et al. Family planning: the unfinished agenda Lancet 2006;368:1810-27.

636. Mulvihill MA, Mailloux L, Atkin W. Advancing policy and research responses to immigrant and refugee women's wealth in Canada. Winnipeg (MB): Centres of Excellence in Women's Health; 2001.

637. Preventive services for adults. 15th ed. Bloomington (MN): Institute for Clinical Systems Improvement; 2009.

638. International Agency for Research on Cancer (IARC). Cervix cancer screening. IARC handbooks for cancer prevention. Vol 10. Lyon (FR): IARC Press; 2005.

639. Yang BH, Bray FI, Parkin DM, et al. Cervical cancer as a priority for prevention in different world regions: an evaluation using years of life lost. Int J Cancer 2004; 109:418-24.

640. Screening for cervical cancer: recommendations and rationale. Washington (DC): US Preventive Services Task Force; 2003. p. 101-9.

641. Laara E, Day NE, Hakama M. Trends in mortality from cervical cancer in the Nordic countries: association with organised screening programmes. Lancet 1987; $1: 1247-9$.

642. Hyman I, Singh PM, Meana M, et al. Physician-related determinants of cervica cancer screening among Caribbean women in Toronto. Ethn Dis 2002;12:268-75.

643. Canadian human mortality database. Montréal (QC): Université de Montréal, Department of Demography; 2007. Available: www.demo.umontreal.ca/chmd/ (accessed 2008 Oct. 10).

644. Screening for cervical cancer. Systematic evidence review no. 25. Rockville (MD): Agency for Healthcare Research and Quality; 2002.

645. McLachlin CM, Mai V, Murphy J, et al.; Cervical Screening Guidelines Development Committee. Cervical screening: a clinical practice guideline. Toronto (ON): Cancer Care Ontario; 2005.

646. Saslow D, Runowicz CD, Solomon D, et al. American Cancer Society guideline for 
the early detection of cervical neoplasia and cancer. CA Cancer J Clin 2002; $52: 342-62$.

647. Rambout L, Hopkins L, Hutton B, et al. Prophylactic vaccination against human papillomavirus infection and disease in women: a systematic review of randomized controlled trials. CMAJ 2007; 177:469-79.

648. Markowitz LE, Dunne EF, Saraiya M, et al. Quadrivalent human papillomavirus vaccine. Recommendations of the Advisory Committee on Immunization Practices $(A C I P)$. Atlanta (GA): Centers for Disease Control and Prevention; 2007. Available: www.cdc.gov/mmwr/preview/mmwrhtml/rr5602a1.htm (accessed 2008 Oct. 2).

649. Barr E, Tamms G, Gellin B, et al. Quadrivalent human papillomavirus vaccine. Clin Infect Dis 2007;45:609-17.

650. Saslow D, Castle PE, Cox JT, et al. American Cancer Society Guideline for human papillomavirus (HPV) vaccine use to prevent cervical cancer and its precursors. $C A$ Cancer J Clin 2007;57:7-28.

651. Arbyn M, Kyrgiou M, Simoens C, et al. Perinatal mortality and other severe adverse pregnancy outcomes associated with treatment of cervical intraepithelial neoplasia: meta-analysis. BMJ 2008;337:a1284.

652. Peto J, Gilham C, Fletcher O. The cervical cancer epidemic that screening has prevented in the UK. Lancet 2004;364:249-56.

653. Quinn M, Babb P, Jones J, et al. Effect of screening on incidence of and mortality from cancer of cervix in England: evaluation based on routinely collected statistics. BMJ 1999:318:904-8.

654. Rieck GC, Tristram A, Hauke A, et al. Cervical screening in 20-24 year olds. $J$ Med Screen 2006;13:64-71

655. Raffle AE, Alden B, Mackenzie EF. Detection rates for abnormal cervical smears: What are we screening for? Lancet 1995;345:1469-73.

656. Brotherton JM, Gold MS, Kemp AS, et al.; New South Wales Health HPV Adverse Events Panel. Anaphylaxis following quadrivalent human papillomavirus vaccination. CMAJ 2008;179:525-33.

657. Miller BA, Kolonel LN, Bernstein L, et al., editors. Racial/ethnic patterns of cancer in the United States 1988-1992. NIH Publ. No. 96-4104. Bethesda (MD): National Cancer Institute; 1996.

658. McDermott S, DesMeules M, Lewis R, et al. Cancer incidence among Canadian immigrants, 1980-1998: results from a national cohort study. J Immigr Minor Health 2011;13:15-26.

659. de Sanjosé S, Diaz M, Castellsagué X, et al. Worldwide prevalence and genotype distribution of cervical human papillomavirus DNA in women with normal cytology: a meta-analysis. Lancet Infect Dis 2007;7:453-9.

660. Clifford GM, Rana RK, Franceschi S, et al. Human papillomavirus genotype distribution in low-grade cervical lesions: comparison by geographic region and with cervical cancer. Cancer Epidemiol Biomarkers Prev 2005;14:1157-64.

661. Mayrand MH, Duarte-Franco E, Rodrigues I, et al.; Canadian Cervical Cancer Screening Trial Study Group. Human papillomavirus DNA versus Papanicolaou screening tests for cervical cancer. N Engl J Med 2007;357:1579-88.

662. Galaal KA, Deane K, Sangal S, et al. Interventions for reducing anxiety in women undergoing colposcopy. Cochrane Database Syst Rev 2007;(3):CD006013.

663. Sasieni PD, Cuzick J, Lynch-Farmery E. Estimating the efficacy of screening by auditing smear histories of women with and without cervical cancer. National Coordinating Network for Cervical Screening Working Group. Br J Cancer 1996;73: 1001-5.

664. Austoker J. Cancer prevention in primary care. Screening for cervical cancer. BMJ 1994;309:241-8.

665. Grewal S, Bottorff JL, Balneaves LG. A Pap test screening clinic in a South Asian community of Vancouver, British Columbia: challenges to maintaining utilization. Public Health Nurs 2004;21:412-8.

666. Matin ML, Lebaron S. Attitudes toward cervical cancer screening among Muslim women: a pilot study. Women Health 2004;39:63-77.

667. Brewer NT, Fazekas KI. Predictors of HPV vaccine acceptability: a theoryinformed, systematic review. Prev Med 2007;45:107-14.

668. Bottorff JL, Balneaves LG, Sent L, et al. Cervical cancer screening in ethnocultural groups: case studies in women-centered care. Women Health 2001;33:29-46.

669. Canadian Immunization Committee. Recommendations on a human papillomavirus immunization program. Ottawa (ON): Public Health Agency of Canada; 2007. Available: www.phac-aspc.gc.ca/publicat/2008/papillomavirus-papillome/papillomaviruspapillome-index-eng.php (accessed 2010 July 21).

670. Murphy KJ, Howlett R. Canadian consensus guidelines on human papillomavirus. Chapter 5: screening for cervical cancer. J Obstet Gynaecol Can 2007;29:S1-60.

671. Morrison BJ. Screening for cervical cancer. In: Canadian Task Force on the Periodic Health Examination. Canadian guide to clinical preventive health care. Ottawa (ON): Health Canada; 1994. p. 870-81.

672. Fiftieth anniversary of the Universal Declaration of Human Rights. New York (NY): United Nations Office of Public Information; 1998. Available: www.un.org/rights/50/decla.htm (accessed 2010 July 21).

673. Caulford P, Vali Y. Providing health care to medically uninsured immigrants and refugees. CMAJ 2006;174:1253-4.

674. Gagnon AJ. The responsiveness of the Canadian health care system towards new- comers. In: Forest PG, Marchildon GP, McIntosh T, editors. Changing health care in Canada. Toronto (ON): University of Toronto Press; 2004. p. 349-88.

675. Bowen S. Language barriers in access to health care. Ottawa (ON): Health Canada; 2000.

676. Reproductive health in refugee situations. Geneva $(\mathrm{CH})$ : United Nations High Commissioner for Refugees; 1999.

677. Saving mothers' lives: reviewing maternal deaths to make motherhood safer - 2003 2005. London (UK): Confidential Enquiry into Maternal and Child Health; 2007. Available: www.cmace.org.uk/getattachment/927cf18a-735a-47a0-9200cdea103781c7/Saving-Mothers-Lives-2003-2005_full.aspx (accessed 2010 Apr. 26).

678. Sword W, Watt S, Krueger P. Postpartum health, service needs, and access to care experiences of immigrant and Canadian-born women. J Obstet Gynecol Neonatal Nurs 2006;35:717-27.

679. Dejin-Karlsson E, Ostergren PO. Country of origin, social support and the risk of small for gestational age birth. Scand J Public Health 2004;32:442-9.

680. Gender-based violence. Minneapolis (MN): Reproductive Health Response in Conflict Consortium; 2007. Available: www.rhrc.org/rhr_basics/gbv.html (accessed 2010 Apr. 26).

681. Vichinsky EP, MacKlin EA, Waye JS, et al. Changes in the epidemiology of thalassemia in North America: a new minority disease. Pediatrics 2005;116:e818-25.

682. Longlois S, Ford J, Chitayat D. Carrier screening for thalassemia and hemoglobinopathies in Canada. Joint SOGC-CCMG Clinical Practice Guideline No. 218. Society of Obstetricians and Gynaecologists of Canada and Canadian College of Medical Geneticists; 2008

683. Giordano PC, Dihal AA, Harteveld CL. Estimating the attitude of immigrants toward primary prevention of the hemoglobinopathies. Prenat Diagn 2005;25:885-93.

684. Innocenti Digest: changing a harmful social convention: female genital mutilation/cutting. Florence (IT); UNICEF Innocenti Research Centre; 2005. Available: www.unicef-irc.org/publications/pdf/fgm eng.pdf (accessed 2010 Apr. 26).

685. Management of pregnancy, childbirth and the postpartum period in the presence of female genital mutilation. Geneva $(\mathrm{CH})$ : World Health Organization; 2001.

686. World Health Organization. Female genital mutilation and obstetric outcome: WHO collaborative prospective study in six African countries. Lancet 2006;367: 1835-41.

687. Female genital mutilation (FGM). Legal prohibitions worldwide. New York (NY): Center for Reproductive Rights; 2007. Available: http://reproductiverights.org/en /document/female-genital-mutilation-fgm-legal-prohibitions-worldwide (accessed 2010 Apr. 26).

688. Chalmers B, Omer-Hashi K. What Somali women say about giving birth in Canada. J Reprod Infant Psychol 2002;20:267-82.

689. Hjelm K, Bard K, Nyberg P, et al. Swedish and Middle-Eastern-born women's beliefs about gestational diabetes. Midwifery 2005;21:44-60.

690. Thornton PL, Kieffer EC, Salabarria-Pena Y, et al. Weight, diet, and physical activity-related beliefs and practices among pregnant and postpartum Latino women: the role of social support. Matern Child Health J 2006;10:95-104.

691. van Eijsden M, van der Wal MF, Bonsel GJ. Folic acid knowledge and use in a multi-ethnic pregnancy cohort: the role of language proficiency. BJOG 2006;113: 1446-51.

692. Haelterman E, Qvist R, Barlow P, et al. Social deprivation and poor access to care as risk factors for severe pre-eclampsia. Eur J Obstet Gynecol Reprod Biol 2003; 111:25-32.

693. Rodriguez MA, Bauer HM, Flores-Ortiz Y, et al. Factors affecting patientphysician communication for abused Latina and Asian immigrant women. $J$ Fam Pract 1998;47:309-11.

694. Ottani PA. Embracing global similarities: a framework for cross-cultural obstetric care. J Obstet Gynecol Neonatal Nurs 2002;31:33-8.

695. Small R, Yelland J, Lumley J, et al. Immigrant women's views about care during labor and birth: an Australian study of Vietnamese, Turkish, and Filipino women. Birth 2002;29:266-77.

696. Thierfelder C, Tanner M, Bodiang CM, et al. Female genital mutilation in the context of migration: experience of African women with the Swiss health care system. Eur J Public Health 2005;15:86-90.

697. Caulford P, Vali Y. Providing health care to medically uninsured immigrants and refugees. CMAJ 2006;174:1253-4.

698. Reyners M. Health consequences of female genital mutilation. Gynecol Prat 2004 $4: 242-51$

699. Health Canada. Family-centred maternity and newborn care: national guidelines. Ottawa (ON): Health Canada; 2000.

700. Reid AJ, Biringer A, Carroll JD, et al. Using the ALPHA form in practice to assess antenatal psychosocial health. Antenatal Psychosocial Health Assessment. CMAJ 1998; 159:677-84

701. Wathen CN, MacMillan HL; Canadian Task Force on Preventive Health Care. Prevention of violence against women: recommendation statement from the Canadian Task Force on Preventive Health Care. CMAJ 2003;169:582-4.

702. ACOG Committee on Obstetrics. ACOG Practice Bulletin No. 78: hemoglobinopathies in pregnancy. Obstet Gynecol 2007;109:229-37. 
Competing interests: Elizabeth Barnett has received grant funding (through her institution) from Sanofi Pasteur and Intercell for work unrelated to this publication; funding for lectures from Merck; royalties from Elsevier; and funding for development of educational presentations from PriMed. She has also received payment for expert testimony. Jennifer Blake is the associate chair of the Department of Obstetrics and Gynecology, Sunnybrook Health Sciences Centre, which has received unrestricted educational grants from pharmaceutical companies in support of educational services; she is vice-chair of Genesis Research Foundation, which receives philanthropic donations from many organizations and which funds research trainees; she is chair of the Canadian Foundation for Women's Health, which receives philanthropic gifts and funds research; and she has served on advisory boards in contraception and has received honoraria for lectures. Marc Deschenes has attended meetings of the Gilead Sciences Advisory Board. Sheila Dunn was on the advisory board of Trimedic in 2008, received speaker fees from Paladin Labs in 2008, received research grants for clinical trials of contraceptives from Organon (ScheringPlough) and provided information (without compensation) to the National Drug Safety and Advisory Committee, in collaboration with Paladin Labs, to support deregulation of emergency contraception in 2007. John Feightner has advised and consulted with the Canadian Task Force on Preventive Health Care. Elizabeth Harvey has served as a national advisory board member for Sanofi Aventis and as a consultant for Lifescan; and her institution receives program funding from the Ontario Diabetes Strategy. In addition, Pfizer, Becton Dickinson, Novo-Nordisk, Eli Lilly, Sanofi Aventis, Bayer, Merck, Astra Zeneca and Bristol Myers Squibb have provided grants to her institution and payments for lectures related to the development and presentation of educational programs about diabetes for primary care providers. Jenny Heathcote has served as a consultant to Axcan Pharma, Gilead Sciences, Hoffmann-La Roche, Merck, Schering-Plough and Tibotec; has received restricted grants from Axcan Pharma, Boehringer Ingelheim, Bristol-Myers Squibb, Debio Pharma, Gilead Sciences, GlaxoSmithKline, Hoffmann-La Roche, Human Genome Sciences, Intercept Pharmaceuticals, Schering-Plough, Tibotec and Vertex; has received unrestricted grants from Axcan Pharma, Boehringer Ingelheim, Gilead Sciences, Hoffmann-La Roche, Schering-Plough and Vertex; and has received speaker's fees from Axcan Pharma, Gilead Sciences, Hoffmann-La Roche, Schering-Plough and Tibotec. Charles Hui has received grant support through his institution, as well as payment for lectures and travel expenses, from Abbott International, and has also served on a scientific advisory board for Novartis. Jay Keystone has received speaker fees and payments for development of educational presentations from GlaxoSmithKline, Sanofi Pasteur and Merck. Stan Kutcher occasionally serves as an expert witness. Noni MacDonald, Section Editor, Population and Public Health, CMAJ, is a coauthor of this article; she was not involved in the vetting of this article before publication. Anne McCarthy has served as an ad hoc consultant on travel medicine to Shorelands Inc. Lavanya Narasiah received speaker fees from GlaxoSmithKline for presentations on travel health. Pierre Plourde received speaker fees from GlaxoSmithKline for presentations on travel health and tropical medicine. Peter Tugwell, chair of the CMA Journal Oversight Committee, is a coauthor of this article; he was not involved in the vetting of this article before publication. David Wong has received educational grants related to hosting the University of Toronto Sheila Sherlock Liver Research Day and the University of Toronto Hepatology Update; he also has received honoraria for educational sessions from Roche, Schering, Gilead, Bristol-Myers Squibb and Novartis (in all instances, the slides used for sessions have been his own). No competing interests declared by Deborah Assayag, Beverly Brockest, Ralf Buhrmann, Giovani Burgos, Glenn Campbell, Andrea Chambers, Angie Chan, Marianne Cheetham, Walter Delpero, Shafik Dharamsi, Arunmozhi Dominic, Ann Duggan, Nancy Durand, Allison Eyre, Anita Gagnon, Jennifer Grant, Christina Greenaway, Doug Gruner, Sinclair Harris, Stewart Harris, Ghayda Hassan, Christine Heidebrecht, Willam Hodge, Danielle Hone, Susan Hum, Praseedha Janakiram, Khairun Jivani, Tomas Jurcik, Kamran Khan, Laurence Kirmayer, Ian Kitai, Srinivasan Krishnamurthy, Susan Kuhn, Robert LaRoche, Carmen Logie, Michelle Martin, Dominique Elien Massenat, Debora Matthews, Barry Maze, Mary McNally, Dick Menzies, Marie Munoz, Félicité Murangira, Amy Nolen, Kevin Pottie, Meb Rashid, Cécile Rousseau, Hélène Rousseau, Andrew Ryder, Amelia Sandoe, Kevin Schwartzman, Jennifer Sears, William Stauffer, Helena Swinkels, Brett Thombs, Patricia Topp, Andrew Toren, Sara Torres, Erin Ueffing, Ahsan Ullah, Sunil Varghese, Bilkis Vissandjee, Vivian Welch, Michel Welt, Wendy Wobeser, Phyllis Zelkowitz, Jianwei Zhong, Stanley Zlotkin.

Contributors: Section 1 (Review): Kevin Pottie, Christina Greenaway, John Feightner, Vivian Welch, Helena Swinkels, Meb Rashid, Lavanya Narasiah, Laurence J. Kirmayer, Erin Ueffing, Noni E. MacDonald, Ghayda Hassan,
Mary McNally, Kamran Khan, Ralf Buhrmann, Sheila Dunn, Arunmozhi Dominic, Anne E. McCarthy, Anita J. Gagnon, Cécile Rousseau, and Peter Tugwell contributed as members of the steering committee and/or lead authors on reviews and played an active role in formulating the recommendations. The other coauthors of the Canadian Collaboration for Immigrant and Refugee Health (see complete list on the title page) provided substantive contributions for the individual systematic reviews as listed below.

Section 2 (Selection of potentially preventable and treatable conditions): Helena Swinkels, Kevin Pottie, Peter Tugwell, Meb Rashid and Lavanya Narasiah contributed to the conception and refinement of the study design and to the analysis and interpretation of the data. Helena Swinkels drafted the initial manuscript, and all authors provided critical revisions and approved the final manuscript submitted for publication.

Section 3 (Evaluation of evidence-based literature): Peter Tugwell, Kevin Pottie, Vivian Welch, Erin Ueffing, Andrea Chambers and John Feightner contributed to the conception, writing and critical revision of the manuscript and approved the final version submitted for publication.

Section 4 (Measles, mumps, rubella, diphtheria, tetanus, pertussis and polio): Christina Greenaway, Marie Munoz, Elizabeth Barnett, Amelia Sandoe, Erin Ueffing, Kevin Pottie, Susan Kuhn and Jay Keystone contributed to the conception and refinement of the study design and the analysis and interpretation of the data. Christina Greenaway drafted the initial draft and all authors provided critical revisions and approved the final manuscript submitted for publication.

Section 5 (Varicella): Christina Greenaway, Meb Rashid, Elizabeth D. Barnett, Amelia Sandoe, Erin Ueffing, Helena Swinkels, Marie Munoz, Kevin Pottie and Jay Keystone contributed to the conception and refinements of the study design and the analysis and interpretation of the data. Christina Greenaway drafted the initial manuscript, and all authors provided critical revisions and approved the final manuscript submitted for publication.

Section 6 (Hepatitis B): Christina Greenaway, Lavanya Narasiah, Pierre Plourde, Erin Ueffing, Kevin Pottie, Marc Deschenes, David K.H. Wong, Susan Kuhn and Jenny Heathcote contributed to the conception and refinement of the study design and to the analysis and interpretation of the data. Christina Greenaway drafted the initial manuscript, and all authors provided critical revisions and approved the final manuscript submitted for publication.

Section 7 (Tuberculosis): Christina Greenaway, Amelia Sandoe, Bilkis Vissandjée, Ian Kitai, Doug Gruner, Kevin Pottie, Erin Ueffing, Wendy Wobeser, Dick Menzies and Kevin Schwartzman contributed to the conception and refinement of the study design and to the analysis and interpretation of the data. Christina Greenaway drafted the initial manuscript, and all authors provided critical revisions and approved the final manuscript submitted for publication.

Section 8 (HIV): Kevin Pottie, Bilkis Vissandjée, Jennifer Grant, Carmen Logie, Ahsan Ullah, Félicité Murangira and Vivian Welch contributed to the conception and refinement of the study design and the analysis and interpretation of the data. Kevin Pottie wrote the initial draft, and all authors provided critical revisions and approved the final manuscript submitted for publication.

Section 9 (Hepatitis C): Christina Greenaway, David K.H. Wong, Deborah Assayag, Marc Deschenes, Chuck Hui, Erin Ueffing, Kevin Pottie, Amelia Sandoe, Meb Rashid and Jenny Heathcote contributed to the conception and refinement of the study design and the analysis and interpretation of the data. Christina Greenaway wrote the initial draft, and all authors provided critical revisions and approved the final manuscript submitted for publication.

Section 10 (Intestinal parasites): Kamran Khan, Christine Heidebrecht, Jennifer Sears, Angie Chan, Meb Rashid, Christina Greenaway, William Stauffer, Lavanya Narasiah and Kevin Pottie contributed to the conception and refinement of the study design and the analysis and interpretation of the data. Kamran Khan drafted the initial manuscript, and all authors provided critical revisions and approved the final manuscript submitted for publication.

Section 11 (Malaria): Anne E. McCarthy, Sunil Varghese, Ann Duggan, Glenn Campbell, Kevin Pottie and Susan Kuhn contributed to the conception and refinement of the study design and the analysis and interpretation of the data. Anne E. McCarthy drafted the initial manuscript and all authors provided critical feedback and approved the final manuscript submitted for publication.

Section 12 (Depression): Laurence J. Kirmayer, Lavanya Narasiah, Andrew G. Ryder, Giovani Burgos, Phyllis Zelkowitz, Kevin Pottie and Stan Kutcher contributed to conception and refinement of the study design and the analysis and interpretation of the data. Laurence J. Kirmayer drafted the initial manuscript and all authors provided critical feedback and approved the final manuscript submitted for publication.

Section 13 (Post-traumatic stress disorder): Cécile Rousseau, Kevin Pottie, Brett D. Thombs, Marie Munoz and Tomas Jurcik contributed to the conception and refinement of the study design and the analysis and interpretation 
of the data. Cécile Rousseau and Kevin Pottie drafted the initial manuscript and all authors provided critical feedback and approved the final manuscript submitted for publication.

Section 14 (Child maltreatment): Ghayda Hassan, Brett D. Thombs, Cécile Rousseau, Laurence J. Kirmayer, John Feightner, Erin Ueffing and Kevin Pottie contributed to the conception and refinement of the study design and the analysis and interpretation of the data. Ghayda Hassan drafted the initial manuscript and all authors provided critical revisions and approved the final manuscript submitted for publication.

Section 15 (Intimate partner violence): Ghayda Hassan, Brett D. Thombs, Cécile Rousseau, Laurence J. Kirmayer, John Feightner, Erin Ueffing and Kevin Pottie contributed to the conception and refinement of the study design and the analysis and interpretation of the data. Ghayda Hassan drafted the initial manuscript, and all authors provided critical revisions and approved the final manuscript submitted for publication.

Section 16 (Diabetes mellitus): Arunmozhi Dominic, Kevin Pottie, Dominique Elien Massenat, Elizabeth Harvey, Jianwei Zhong and Stewart B. Harris contributed to the conception and refinement of the study design and the analysis and interpretation of the data. Arunmozhi Dominic drafted the initial manuscript, and all authors provided critical revisions and approved the final manuscript submitted for publication.

Section 17 (Iron-deficiency anemia): Kevin Pottie, Andrea Chambers, Beverly Brockest, Vivian Welch and Stanley Zlotkin contributed to the conception and refinement of the study design and the analysis and interpretation of the data. Kevin Pottie drafted the initial manuscript and all authors provided critical revisions and approved the final manuscript submitted for publication.

Section 18 (Dental disease): Mary McNally, Debora Matthews, Kevin Pottie, Barry Maze, Shafik Dharamsi, Helena Swinkels, Khairun Jivani, Vivian Welch and Ahsan Ullah contributed to the conception and refinement of the study design and the analysis and interpretation of the data. Mary McNally drafted the initial manuscript, and all authors provided critical revisions and approved the final manuscript submitted for publication.

Section 19 (Vision health): Ralf Buhrmann, Andrew Toren, William Hodge, Allison Eyre, Michelle Martin, Walter Delpero, Robert Laroche and Kevin Pottie contributed to the conception and refinement of the study design and the analysis and interpretation of the data. Ralf Buhrmann and Andrew Toren drafted the initial manuscript and all authors provided critical revisions and approved the final manuscript submitted for publication.

Section 20 (Contraception): Sheila Dunn, Praseedha Janakiram, Jennifer Blake, Susan Hum, Marianne Cheetham, Vivian Welch and Kevin Pottie contributed to the conception and refinement of the study design and the analysis and interpretation of the data. Sheila Dunn led the evidence review and all authors provided critical revisions and approved the final manuscript submitted for publication.

Section 21 (Cervical cancer): Kevin Pottie, Amy Nolen, Patricia Topp, Sara Torres, Vivian Welch and Nancy Durand contributed to the conception and refinement of the study design and the analysis and interpretation of the data. Amy Nolen and Kevin Pottie drafted the initial manuscript and provided critical revisions and approved the final manuscript for publication.

Section 22 (Pregnancy): Anita J. Gagnon, Hélène Rousseau, Michel Welt, Sinclair Harris, Danielle Hone, Srinivasan Krishnamurthy and Kevin Pottie contributed to the conception, writing and critical revision of the manuscript and approved the final version submitted for publication.

Funding: The Public Health Agency of Canada (PHAC) contributed funding for the development and publication of reviews of the scientific evidence on select topics related to PHAC programs of work. The conclusions and recommendations made in the guidelines were independently developed by the Canadian Collaboration for Immigrant and Refugee Health. The views expressed in this report are the views of the authors and do not necessarily reflect those of PHAC or any other agency that provided funding support. In addition to the support from PHAC, the Canadian Collaboration for Immigrant and Refugee Health acknowledges funding support for this project from the Canadian Institutes of Health Research (Institute of Health Services and Policy Research), the Champlain Local Health Integrated Network and the Calgary Refugee Program. The Calgary Refugee Program, the Champlain Local Health Integrated Network and the Canadian Institutes of Health Research (Canadian Institute of Health Services and Policy Research) contributed to dissemination of the guidelines. The Office of the Chief Dental Officer, Health Canada, provided support for the evidence review on dental health (section 18). Anita J. Gagnon's participation in the project was supported by McGill University through a William Dawson Scholarship. Christina Greenaway receives salary support from the Fonds de la recherche en santé du Québec (FRSQ) through the Chercheur Boursier Clinicien. Carmen Logie receives funding support from the Social Sciences and Humanities Research Council of Canada through a doctoral fellowship. Dick Menzies receives salary support from the FRSQ through the Chercheur National program. Brett Thombs is supported by a New Investigator Award from the Canadian Institutes of Health Research and an Établissement de Jeunes Chercheurs award from the FRSQ.

Acknowledgements: The authors acknowledge the participation of the following primary care practitioners: Jessica Audley (Toronto), Rolando Barrios (Vancouver), Ricardo Batista (Ottawa), Denis Bédard (Québec), Angela Carol (Hamilton), Juan Carlos Luis Chirgwin (Montréal), Tyler Curtis (Toronto), Gilles de Margerie (Montréal), Mike Dillon (Winnipeg), Pierre Dongier (Montréal), Omar Ezzat (Newfoundland and Labrador), Lynn Farrales (Vancouver), Mariella Ferreyra (Toronto), Susanne Fremming (Vancouver), Carol Geller (Ottawa), Réka Gustafson (Vancouver), Susan Hoffman (Toronto), Lanice Jones (Calgary), Valerie Krinke (Edmonton), Britta Laslo (Hamilton), Kay Lee (Ottawa), Lise Loubert (Vancouver), Kathie McNally (Charlottetown), Marie-Jo Ouimet (Montréal), Bill Pegg (Toronto), Eva Purkey (Kingston), Leslie Rourke (St. John's), Millaray Sanchez (Ottawa), Kerry Telford (Vancouver), Gail Webber (Ottawa) and Ed White (Charlottetown). The authors also thank immigrant community stakeholder partners Yvonne Chiu and Lucenia Ortiz, who provided feedback on behalf of the Edmonton Multicultural Health Brokers Cooperative.

The authors thank Patricia Thille, Jennifer Creer, Roo Deinstadt and Ayesha Ratnayake for writing and formatting support, assistance in developing the section on cultural considerations and assistance with the literature reviews; Karin Okrainec for her assistance in launching the Delphi process; other staff members at the Institute of Population Health, University of Ottawa - Erika Espinosa, Belinda Smith, Liz Lacasse, Maria Benkhalti, Leanne Idzerda, Govinda Dahal and Jessie McGowan - for their general project support and assistance in retrieving articles; Lynn Dunikowski, MLS, Director of Library Services, College of Family Physicians of Canada, for expert medical librarian support and for designing the literature searches; Jocelyne Andrews and Teodora Constantinu for conducting certain of the bibliographic searches; Kay Berckmans and Antonella Clerici for secretarial support; Hilary Elkins, Lisa Merry and Diane Habbouche for general support; Sarah McDermott for feedback on the evidence review for diabetes mellitus; Glenda Dare for helpful comments on an earlier version of the HIV review; Sudeep Chaklabanis for assistance in coordinating the review process for depression; Michaela Beder, Cristiano Martello, Vincenzo Spigonardo and Lorin Young for help in reviewing the literature on depression; Radmika Santhanam-Martin and Yasir Khan for assistance with the evidence review for post-traumatic stress disorder; Megan Galeucia and Vanessa Lecompte for help in reviewing the literature on child maltreatment; Juan Carlos Luis Chirgwin for helpful feedback on earlier drafts of the evidence reviews; Rimma Orneman, Megan Galeucia and Mylène Boivin for contributions to the literature review on intimate partner violence; and Sheila Innis for helpful feedback on an earlier draft of the evidence review for iron-deficiency anemia. The authors also thank Holger Schünemann, Nancy Santesso, Andy Oxman and Gordon Guyatt for their help with GRADE methods and presentation of findings. 